\title{
Advances in Grasping and Vehicle Contact Identification: Analysis, Design and Testing of Robust Methods for Underwater Robot Manipulation
}

by

\author{
Edward Ramsey Snow \\ B.S., Mechanical Engineering, Cornell University (1986) \\ S.M., Mechanical Engineering, MIT(1994)
}

Submitted in partial fulfillment of the requirements for the degree of

Doctor of Philosophy

at the

\section{MASSACHUSETTS INSTITUTE OF TECHNOLOGY}

and the

WOODS HOLE OCEANOGRAPHIC INSTITUTION

May 1999

(c) Edward Ramsey Snow, 1999. All rights reserved.

The author hereby grants to MIT and WHOI permission to reproduce paper and electronic copies of this thesis in whole or in part, and to distribute them publicly.

Author

Department of Applied Ocean Physics and Engineering, WHOI

Department of Mechanical Engineering, MTT

May, 1999

Certified by $\ldots \ldots \ldots \ldots \ldots \ldots \ldots \ldots \ldots$

U Dana R. Yoerger

Associate Scientist, Department of Applied Ocean Physics and Engineering, WHOI

Accepted by
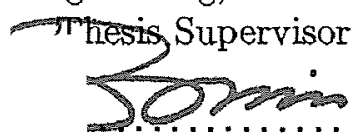

Protessor Ain A. Sonin

Chair, Mechanical Engineering Department Graduate Committee, MIT

Accepted by ...

.........................................

Chair, Joint Committee for Oceanography Applied Ocean Science and Engineering

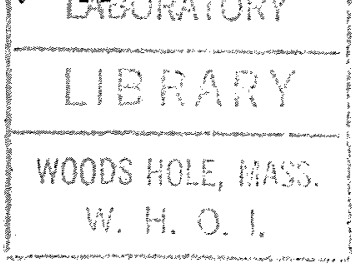

WHOI 
2 


\title{
Advances in Grasping and Vehicle Contact Identification: Analysis, Design and Testing of Robust Methods for Underwater Robot Manipulation
}

by

\author{
Edward Ramsey Snow
}

\author{
Submitted to the \\ Department of Mechanical Engineering \\ on May, 1999, in partial fulfillment of the \\ requirements for the degree of \\ Doctor of Philosophy
}

\begin{abstract}
This thesis focuses on improving the productivity of autonomous and telemanipulation systems consisting of a manipulator arm mounted to a free flying underwater vehicle.

Part I minimizes system sensitivity to misalignment by developing a gripper and a suite of handles that passively self align when grasped. After presenting a gripper guaranteed to passively align cylinders we present several other self aligning handles. The mix of handle alignment and load resisting properties enables handles to be matched to the needs of each task. Part I concludes with a discussion of successful field use of the system on the Jason Remotely Operated Undersea Vehicle operated by the Woods Hole Oceanographic Institution.

To enable the exploitation of contact with the environment to help stabilze the vehicle, Part II develops a technique which identifies the contact state of a planar vehicle interacting with a fixed environment. Knowing the vehicle geometry and velocity we identify kinematically feasible contact points, from which we construct the set of feasible contact models. The measured vehicle data violates each model's constraints; we use the asociated violation power and work to select the best overall model. Part II concludes with experimental confirmation of the contact identification technique's efficacy.
\end{abstract}




\section{Acknowledgments}

I owe a great many people for their help and patience while I worked on this thesis. Foremost among these people is my advisor, Dana Yoerger. Dana has been my academic advisor since I started my masters thesis. In addition to guidance and support, Dana allowed me almost complete freedom in choosing the topics I pursued in both theses. Such a gift is increasingly rare these days given the changes that have occurred in the way projects are funded. I thank you for these opportunities and for the belief you have shown in me by bestowing them upon me.

Just after finishing my masters thesis I developed a severe case of tendinitis in both wrists. The following year and a half was a very difficult time for me; the injury limited my productivity to the point where it was unclear that I would be able to continue a career in my chosen field. Dana believed in me and, by continuing to support me, gave me the time I needed to heal and to adapt to what appears to be a permanent condition. Dana, I will forever be in debt you; you helped me save my career.

Dana was not the only one who saw me through this time. I'm also forever indebted to the Education Office for their support, their belief in me and their flexibility in permitting me to complete my studies. John Farrington, Jake Pearson, Marcy Simon, Stella Callagee, and Julia Westwater all showed me great compassion, both professionally and personally, in giving me the time and resources I needed to complete my studies. Without their help you would not be reading this document right now. The same holds for Mark Grosenbaugh, the educational coordinator for the AOPE department. Mark, alongside Dana, has been a tireless advocate on my behalf and has always given me encouragement and good advice whatever the circumstances.

I am very grateful to each of my committee members; Ken Salisbury, KamalYoucef Toumi and Louis Whitcomb. Through their questions and advice each has not only broadened my understanding of my work but improved my approach to solving problems as well. Thank you all. I owe a special thanks to Ken. I first met 
Ken in 1986 and since that time he has been both a role model and a guardian angel; his comments on my behalf played a major role in my getting an job interview at JPL and in my getting into MIT. Ken helped me launch the career that Dana helped me save. Thanks again to both of you.

I feel very privileged to have worked with the members of the Deep Submergence Laboratory while performing my research; Andy Bowen, Will Sellers, Skip Gleason, Tom Crook, Bob Elder, Steve Lerner, John Howland, P.J., Matt Naiman, Steve Gegg. I have learned a great deal from these individuals and I thank them, not only for the opportunities they've given me to contribute but for the respect they've shown me by doing so. I didn't come to MIT to get a PhD; I came to become a better engineer. A great deal of whatever improvement I have made is directly attributable to my interaction with these individuals. The remainder is due to the inspiration, infectious enthusiasm for design and course material provided by Professors Warren Searing and Alex Slocum while teaching their graduate design course at MIT.

Thanks to Leslie and Joan for a smile and a caring inquiry each time I called or stuck my head in the ME graduate office over the many years I was at MIT. Thanks also to Larry Flick for all his help filling out P.O.s and for all the hours I know he must have spent with Dana figuring out how to support me. Additional thanks to all the DSL secretaries over the years (Nancy, Cindy, Beven, Anita) for their help with administrative details and with trying to track down Dana and whatever corner of the world he happened to be in at the time.

I owe a special thanks to Jason Gobat. If Jason were a company I would advise everyone I know to invest in him. Jason listened to innumerable explanations and questions and provided uniformly valuable input and insight on topics related to my thesis. He also spent untold hours helping me learn how to program in $\mathrm{C}$ and lending me the benefit of his experience in data aquisition and hardware I/O. All while pursuing his own $\mathrm{PhD}$ thesis. Thanks Jason, you were a huge help.

Special thanks as well to my housemate Debbie who kept me sane as we both 
converged on completing our degrees. The existence of hell is justified by the quality of the friendships one makes while passing through it.

As grateful as I am for the debts I have listed above, none even remotely compare to that I owe my parents. From their hard work have come my opportunities. From their parenting, genes and love have come the values, abilities and self confidence required to transform opportunities into accomplishments. While all individuals are unique, we are not created in a vacuum; to get me mix equal parts of Dad, Mom, and something new (and throw in a dash of my big sister Lydia). There is no achievement in my life in which they are not equal partners. Thank you Mom and Dad.

Speaking of partners, my final thanks go to my love, Kathy, for her patience, faith and love throughout this process. I am a better person because of you and I look forward to the opportunities awaiting us together. 


\section{Contents}

$\begin{array}{lll}1 & \text { Introduction } & 27\end{array}$

1.1 Effects of misalignment . . . . . . . . . . . . . . . 28

1.2 General Problem Statement . . . . . . . . . . . . . . . . . . 29

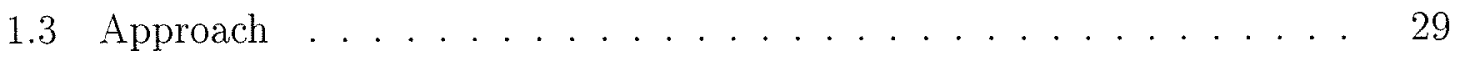

I Minimizing System Sensitivity to Misalignment 31

2 Devising a Misalignment Tolerant Subsea Grasping System 32

2.1 Abstract . . . . . . . . . . . . . . . . . . . . . . . . . . . . 32

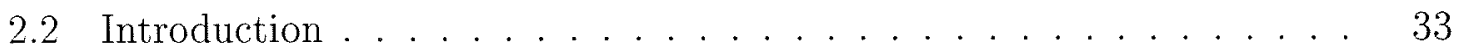

2.3 Grasping and misalignment $\ldots \ldots \ldots \ldots \ldots$

2.4 Issues concerning misalignment tolerant gripper/handle systems . . 37

2.5 Mislignment Tolerant Grasping of Cylinders . . . . . . . . . 38

2.6 Expanding the actuator-orthogonal load space . . . . . . . . . 43

2.7 Additional positively locating handles . . . . . . . . . . . 45

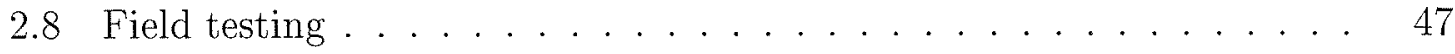

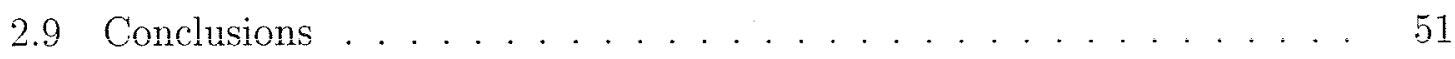

II Minimizing System Contributions to Misalignment 55

$\begin{array}{lll}3 & \text { Introduction } & 56\end{array}$ 
3.1 Related Work . . . . . . . . . . . . . . . . . . . 58

3.1.1 Control of Free-Flying Manipulation Systems . . . . . . . 58

3.1 .2 Compliant Motion Control . . . . . . . . . . . . . . . 58

3.1 .3 Bracing . . . . . . . . . . . . . . . . . 60

3.1 .4 Intrinsic Contact Sensing . . . . . . . . . . . . . 62

3.1 .5 Fixturing . . . . . . . . . . . . . . . . 62

3.1 .6 Contact Identification $\ldots \ldots \ldots \ldots . \ldots \ldots$

3.2 Problem Statement . . . . . . . . . . . . . . . . . . . . . . 64

3.3 Contributions of Part II f . . . . . . . . . . . . . . . 64

3.4 Overview . . . . . . . . . . . . . . . . 65

3.4 .1 Synopsis of Approach . . . . . . . . . . . . . . . 65

3.4 .2 Guide to Part II . . . . . . . . . . . . . . . . . . . . 67

3.4 .3 Assumptions . . . . . . . . . . . . . . . . . . 68

4 Identifying Kinematically Feasible Candidate Contact Points $\quad 70$

4.1 Introduction . . . . . . . . . . . . . . . . 70

4.2 Properties of Planar Rigid Body Contact . . . . . . . . . . . 71

4.3 Zero-Normal-Velocity (znv) Points . . . . . . . . . . . . . . . 72

4.3.1 The Instantaneous Center of Rotation . . . . . . . . . 73

4.3.2 Locating znv points on straight line boundary segments . . . 75

4.3.3 Locating znv points on circular arc boundary segments . . . 77

4.3.4 Locating znv points on composite curves . . . . . . . . 80

4.4 Experimental Results . . . . . . . . . . . . . . . . . 80

4.4.1 Case 1: One Point Contact with No Slip (Pure Rotation) . . . 82

4.4.2 Case 2: One Point Contact with Slip (Rotation and Translation) 82

4.4.3 Case 3: Two Point Contact (Pure Translation) . . . . . . . . 85

4.4.4 Case 4: Two Point Contact (Rotation and Translation) . . . . 85

4.5 Conclusion . . . . . . . . . . . . . . . . . . 86 
5 Constructing Kinematically Feasible Candidate Contact Models $\quad 89$

5.1 Introduction . . . . . . . . . . . . . . . . . . . . . 89

5.2 Measuring the Net Contact Induced Force and Moment . . . . . . . 90

5.3 Model Classes . . . . . . . . . . . . . . . . . . . . . . . . . 92

5.4 The Permissible Force Space . . . . . . . . . . . . . . . . . 94

5.5 The Permissible Velocity Space . . . . . . . . . . . . . 95

5.6 The Permissible Motion Space . . . . . . . . . . . . . . . . . 97

5.7 Model Constraint Equations . . . . . . . . . . . . . . . . . . . . 99

5.7 .1 Unconstrained Model . . . . . . . . . . . . . . . . . . . . 99

5.7 .2 One Point Contact Models with Slip . . . . . . . . . 100

5.7.3 One Point Contact Models with No Slip . . . . . . . . . . 103

5.7.4 Two Point Contact Models . . . . . . . . . . . . . . 106

5.7.5 Fully Constrained Model . . . . . . . . . . . . . . . . 109

5.8 Conclusion . . . . . . . . . . . . . . . . . . . . . . . 109

6 Identifying the Best Candidate Contact Model in Each Class 111

6.1 Introduction . . . . . . . . . . . . . . . . . . 111

6.1 .1 Model Feasibility . . . . . . . . . . . . . . . . . . . 112

6.1 .2 Model Consistency . . . . . . . . . . . . . . . . . 112

6.1 .3 Approach . . . . . . . . . . . . . . 115

6.2 Contact Point Reaction Loads . . . . . . . . . . . . . . . 115

6.2.1 Problems with a Direct Least Squares Solution for $\mathbf{f}_{c} \ldots \ldots 116$

6.2 .2 Violation Power . . . . . . . . . . . . . . . . . 117

6.2.3 Modified Least Squares Solution . . . . . . . . . . . . 118

6.3 Permissible and Impermissible Components of the Measured Force . 120

6.3 .1 Computing $\mathcal{F}_{P} \ldots \ldots \ldots \ldots \ldots \ldots \ldots$

6.3 .2 Computing $\mathcal{F}_{I} \ldots \ldots \ldots \ldots \ldots \ldots \ldots \ldots$

6.3.3 Orthogonality of $\mathcal{F}_{P}$ and $\mathcal{F}_{I} \ldots \ldots \ldots \ldots \ldots$ 
6.4 Why the Technique Is Limited to Selecting the Best Candidate Model in Each Class . . . . . . . . . . . . . . . . . . . . . . . . . 122

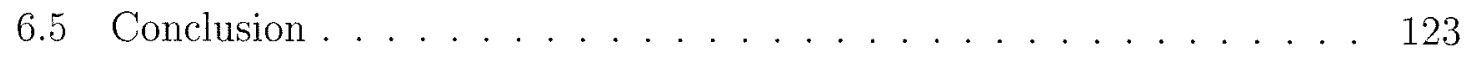

7 Identifying the Best Overall Model $\quad 124$

7.1 Incremental Motion of a Model's Contact Points . . . . . . . . . . . . 125

7.1.1 Problems with a Direct Least Squares Solution for $\Delta \mathrm{x}_{c} \ldots \ldots 125$

7.1 .2 Violation Energy . . . . . . . . . . . . . . 126

7.1 .3 Modified Least Squares Solution . . . . . . . . . . . . . . . . 127

7.2 Permissible and Impermissible Components of the Incremental Motion Vector . . . . . . . . . . . . . . . . . . 129

7.2 .1 Computing $\Delta \boldsymbol{X}_{P} \ldots \ldots \ldots \ldots . \ldots \ldots$

7.2 .2 Computing $\Delta \mathcal{X}_{I} \ldots \ldots \ldots \ldots$

7.3 Conclusion . . . . . . . . . . . . . . . . . . 130

8 Experimental Verification 131

8.1 Introduction . . . . . . . . . . . . . . . . . 131

8.2 Experimental Apparatus . . . . . . . . . . . . . . . . 131

8.3 Comments on Measuring the Accuracy of the Identification Technique 133

8.4 Using the Violation Power Based Consistency Measure $r_{p_{v}}$ to Select the Best Model in a Class . . . . . . . . . . . . . . . . . . . . . . 134

8.4.1 Case 1: One Point Contact with No Slip (Fixed Point Rotation) 134 8.4.2 Case 2: One Point Contact with Slip (Rotation and Translation) 136 8.4.3 Case 3: Two Point Contact (Pure Translation) . . . . . . . . 138 8.4.4 Case 4: Two Point Contact (Rotation and Translation) . . . 140 8.5 Using the Violation Energy to Select the Best Best-of-Class Model . . 140

8.5.1 Case 1: One Point Contact with No Slip (Fixed Point Rotation) 142

8.5.2 Case 2: One Point Contact with Slip (Rotation and Translation) 142

8.5.3 Case 3: Two Point Contact ( Pure Translation) . . . . . . . . 144 
8.5.4 Case 4: Two Point Contact (Rotation and Translation) . . . 146

8.5.5 Case 5: Mixed Contact . . . . . . . . . . . . . 149

8.6 Hazards of Inequality Based Tests . . . . . . . . . . . . . . . . . . . . 149

8.6.1 Quasistatic Feasibility Test . . . . . . . . . . . . . . 149

8.6.2 Direction of Friction Force Test . . . . . . . . . . . . . 152

8.6.3 Candidate Contact Point Domain Test . . . . . . . . . . . . . 152

8.7 Sensitivity to Assumed Value of Dynamic Coefficient of Friction . . 152

8.8 Numerical Investigation of the Uniqueness of Apparently Equivalent Models ....................... 153

8.9 Conclusion . . . . . . . . . . . . . . . . 155

9 Conclusions $\quad 156$

9.1 Part I: Misalignment Tolerant Grasping . . . . . . . . . . . . . . . . 156

9.1 .1 Summary ...................... 156

9.1.2 Future Work . . . . . . . . . . . . . . . . . 157

9.2 Part II: Contact Identification . . . . . . . . . . . . . . . . 158

9.2 .1 Summary .......................... 158

9.2 .2 Future Work . . . . . . . . . . . . . . . . . . . . 159

9.2.3 Use of the Technique with Concave Objects . . . . . . . . 161

9.3 Conclusion . . . . . . . . . . . . . . . . 162

A Experimental Apparatus $\quad 164$

A.1 Justification for building an experimental testbed . . . . . . . . . 164

A.2 Form of the Apparatus . . . . . . . . . . . . . 165

A.3 Scaling . . . . . . . . . . . . . . . . . . . . . . . . 168

A.3.1 Dimensional Analysis . . . . . . . . . . . . . 168

A.3.2 Parameter values for the Jason ROV . . . . . . . . . . . . 173

A.4 Kinematics ..................... . . 173

A.4.1 Geometry of an open circuit cable drive . . . . . . . . . . . 173 
A.4.2 Inverse Kinematics . . . . . . . . . . . . . 175

A.4.3 Forward Kinematics . . . . . . . . . . . . . . 177

A.4.4 The Jacobian . . . . . . . . . . . . . . . . . . . 180

A.5 Statics . . . . . . . . . . . . . . . . . 182

A.5.1 Ensuring Positive Cable Tensions . . . . . . . . . . . . 182

A.6 Transmission Non-idealities . . . . . . . . . . . . . . . . . . 183

A.6.1 Stiffness of Open Circuit Cable Drive . . . . . . . . . 184

A.6.2 Resonant Frequencies . . . . . . . . . . . . . . . . . . 186

A.6.3 Strum Frequencies . . . . . . . . . . . . . . 190

B Case 1: One Point Contact with No Slip ( Pure Rotation) 191

C Case 2: One Point Contact with Slip ( Rotation and Translation ) 196

D Case 3: Two Point Contact ( Pure Translation ) 201

E Case 4: Two Point Contact ( Rotation and Translation ) 206

F Case 5: Two Point Contact ( Rotation and Translation ) 211

G Consistency Measure and Dimenional Analysis 216

$\mathrm{H}$ Reaction loads for two point contact models, assuming $\mu_{d}$ unknown219

I Mathematical Notation for Part II 222 


\section{List of Figures}

2-1 1 DOF gripper which positively locates grasped cylinders: a. during capture, plan view. b. cylinder fully grasped, plan view. c. cylinder fully grasped, isometric view showing that each of the grippers "fingers" actually consists of two plates separated in the z-direction. . . . . .

2-2 Grasping geometry showing the cylinder's final position and the cylinder's capture range (shaded area). Successful grasping is guaranteed if the cylinder's center lies in this region and the external loads meet the conditions discussed in the text. . . . . . . . . . . . . .

2-3 Forces acting on a cylinder in contact with both fingers as the gripper closes. a. Geometry and external forces ( i.e. finger closure torque $M$ and a purely vertical external force acting at the cylinder center $F$ ) b. detail of frictional, normal and external loads acting on the cylinder ( $\theta$ is the angle between a line connecting the contacting surface of the

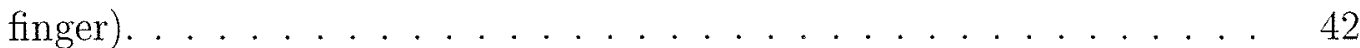


2-4 The minimum dimensionless (quasi-static) outward force applied to a cylinder during closure of the gripper as a function of the dimensionless radius of the cylinder to be grasped and the associated static coefficient of friction. Combinations for which $\frac{F D}{M}>0$ are guaranteed, in the absence of external forces, to reach the desired final position/orientation. Combinations for which $\frac{F D}{M} \leq 0$ indicate that the cylinder reaches static equilibrium (i.e. jams) prior to reaching the desired position/orientation. . . . . . . . . . . .

2-5 Modified gripper design a. H-handle during capture b. H-handle fully grasped, plan view. c. H-handle, isometric view . . . . . . . . 46

2-6 Additional handles which positively locate in a. 6 D.O.F., b. 5 D.O.F., c. 4 D.O.F. and d. 6 D.O.F.. . . . . . . . . . . . . . . .

2-7 Elevator platform loaded with core tubes, water samplers and bioboxes. Elevator platforms transfer tools and samples between the surface and the work site, enabling the vehicle to remain on site indefinitely. The vehicle operator uses the manipulator to transfer all material between the vehicle and the elevator . . . . . . . . . . 51

2-8 Gripper grasping sulfide rock sample. The operator uses the gripper to break samples off from the vent structure or to pick up samples lying on the bottom . . . . . . . . . . . . . . . . . .

2-9 Gripper collecting mussel samples. Mussel samples are subsequently placed in a bio-box on the vehicle and eventually the full bio-box is transferred back to the elevator platform and replaced with an empty

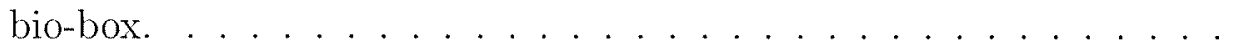

2-10 Gripper grasping core tube sampler. Plunging the sampler into the sediment collects a cylindrical sample of bottom material. The full core tube is placed into a sleeve fit holster for eventual return to an elevator platform. (Outline added by authors.). . . . . . . . . . . 
2-11 Gripper using an X-handle to enlarge plume orifice. Vent orifices are enlarged to facilitate sampling of the plume water. . . . . . . . 53

2-12 Gripper grasping water sampler. An X-handle attached to the sampler permits easy, secure grasping of the sampler. After placing the ends of the sampling tubes into the enlarged vent orifice the operator triggers the bottle to collect a sample of the $300+$ degree Centigrade plume water. (Outline added by authors). . . . . . . . . . . .

3-1 Remotely Operated Vehicle (ROV) based manipulator being used to perform a sampling task. In one case the vehicle hovers while the task is performed, in the other the vehicle exploits contact between the vehicle and a structure in the environment to stabilize the vehicle against manipulator reaction loads. . . . . . . . . . . . . .

3-2 Incorporation of a contact identification system with the hybrid control framework. Note that the identification system plays the role of a sensor, i.e. it simply makes information available, it does not directly update any system parameters. . . . . . . . . . . . .

4-1 (a) Two planar rigid bodies in contact. (b) Tangent vector and outward facing normal at contact point $p_{B}$ on body B. (c) Tangent vector and outward facing normal at contact point $p_{A}$ on body A. . . . . . . .

4-2 The number in each region indicates the number of points on the vehicle perimeter (heavy black line) for which $v_{n}=0$ when the instantaneous center of rotation (ICR) lies within that region. For example, when the ICR is at the indicated position there are eight points which have zero velocity in the direction normal to the local surface tangent. For the given vehicle state and geometry, these are the only possible points of contact with stationary objects in the environment. . . . . . . . . 
4-3 There are generally two znv points (points A and B) associated with a circular arc boundary segment, these being the intersections of the line drawn from the ICR to the arc center C with the arc's underlying circle. Only solutions which lie within the domain of the arc qualify as candidate contact points. An intersection point lies in the domain of the arc if the vector $\mathbf{r}_{p}$ from the arc center $\mathrm{C}$ to the point satisfies the

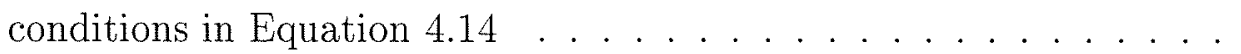

4-4 The Air Table Vehicle Simulator (ATVS) system consists of a 0.145 by 0.29 meter, air bearing supported vehicle which moves freely over the surface of a one square meter glass topped table surface. Four miniature steel cables couple the motion of the vehicle to that of the four motors mounted to the corners of the table. In general, the position and orientation of the vehicle is controlled by coordinating the motion of the four motors. The position and orientation, as well as the linear and angular velocities, of the vehicle are determined from the motor shaft positions and angular velocities as measured by optical encoders mounted to the motor shafts. For all the experiments performed in this chapter, however, the vehicle was moved by hand, i.e. the actuators were only used to maintain tension in the cables. This system is described in greater detail in Appendix A . . . . . . . . . .

4-5 Case 1 Experiment: Edge 5 of the vehicle was brought into contact with corner c1 of a fixed object in the environment. The vehicle was then rotated about this corner such that little or no slip occurred. Initial rotation was in the counter clockwise direction, then the rotation was briefly stopped and then reversed, again maintaining minimal slip at

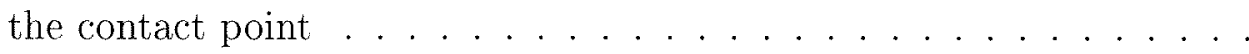


4-6 Case 1 Experiment: Distance between the computed location of the znv point for edge 5 and the known position of corner c1. During rotation the znv point is seen to track the actual contact point within an accuracy of roughly $\pm 5 \mathrm{~mm}$. The vehicle velocity goes to zero at times $t_{1}$ and $t_{2}$. Very near these times the magnitude of the vehicle velocity is too small to yield reliable readings from the velocity sensors, leading to poor estimates of the znv point location. As soon as the vehicle begins to move again, the error returns to the $\pm 5 \mathrm{~mm}$ range. .

4-7 Case 2 Experiment: Edge 5 of the vehicle was brought into contact with corner cl of a stationary object. The vehicle was then moved such that it was in sliding contact with the corner, i.e. the motion combined both rotation and translation of the vehicle relative to the contact point. . . . . . . . . . . . . .

4-8 Case 2 Experiment: Distance between the computed location of the znv point for edge 5 and the known position of corner $c 1$. The vehicle was in contact with the corner until time $t_{1}$, at which point contact was broken. The tracking accuracy between the znv point and the known contact location is within $10 \mathrm{~mm}$ for this trial. . . . . . . . . .

4-9 Case 3 Experiment: Edge 5 of the vehicle was brought into full contact with a flat wall. This contact was maintained throughout the trial as the vehicle was translated first upward and then downward back to its approximate initial position. The wall was a smooth, rectangular block of 6061 aluminum. . . . . . . . . . . . . . . . .

4-10 Case 4 Experiment: Arc 4 and edge 5 were initially in contact with corners $\mathrm{c} 1$ and $\mathrm{c} 2$ (we refer to this contact state as $(4,5)$ ). The vehicle was then moved such that the contact state transitioned to $(3,5)$ and

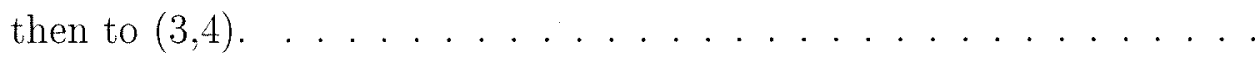


4-11 Upper plot: Distance between corner c1 and the znv points for edge 3, arc 4 and edge 5. Lower plot: Distance between corner c2 and the znv

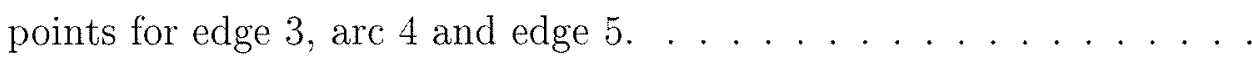

5-1 Possible design of an instrumented rigid frame to measure the net force and moment applied to the vehicle as a result of contact with objects in the environment. The frame is the only part of the vehicle which can come into contact with the environment. Three pin-jointed, uniaxial force sensors would rigidly affix the frame to the main vehicle structure. The three components of the net force and moment applied to the vehicle $f_{x}, f_{y}$ and $m_{z}$ can be determined from the force measurements from the three load cells. . . . . . . . . . . . . . . .

5-2 (a) Vehicle with a single, sliding point of contact with a fixed rigid body in the environment. (b) Absolute velocity of the point on the vehicle which is in contact in contact frame coordinates. (c) Forces acting at the contact point in contact frame coordinates. When the contact involves sliding, the tangential component $f_{t}=\mu_{d} \operatorname{sign}\left(v_{t}\right) f_{n}$, so describing the contact force requires only one independent parameter: $f_{n}$

5-3 (a) Vehicle with a single, non-sliding point of contact with a fixed rigid body in the environment. (b) Absolute velocity of the point on the vehicle which is in contact in contact frame coordinates. (c) Forces acting at the contact point in contact frame coordinates. When the contact point is non-sliding, the tangential and normal components $f_{t}$ and $f_{n}$ are independent parameters. . . . . . . . . . 104 
5-4 Vehicle with a two sliding points of contact with a fixed rigid bodies in the environment. Since sliding occurs at both contacts, the tangential components of the contact forces are $f_{t_{i}}=\mu_{d} \operatorname{sign}\left(v_{t_{i}}\right) f_{n_{i}}$ and $f_{t_{j}}=\mu_{d} \operatorname{sign}\left(v_{t_{j}}\right) f_{n_{j}}$, i.e. describing the contact forces requires two independent parameter: $f_{n_{i}}$ and $f_{n_{j}} \ldots \ldots \ldots$

8-1 The Air Table Vehicle Simulator (ATVS) system consists of a 0.145 by 0.290 meter, air bearing supported vehicle which moves freely over the surface of a one square meter glass topped table surface. Four miniature steel cables couple the motion of the vehicle to that of the four motors mounted to the corners of the table. In general, the position and orientation of the vehicle is controlled by coordinating the motion of the four motors. The position and orientation, as well as the linear and angular velocities, of the vehicle are determined from the motor shaft positions and angular velocities as measured by optical encoders mounted to the motor shafts. For all the experiments performed in this thesis, however, the vehicle was moved by hand i.e. the actuators were only used to maintain tension in the cables. This system is described in greater detail in Appendix A . . . . . . . . . . . .

8-2 Case 1 Experiment: Edge 5 of the vehicle was brought into contact with corner $\mathrm{c} 1$ of a fixed object in environment. The vehicle was then rotated about this corner such that little or no slip occurred at the contact point. Initial rotation was the counter clockwise direction, then the rotation was reversed, bringing the vehicle (approximately) back to its original position. The obstacle was a square bar of 6061 aluminum alloy. . . . . . . . . . . . . . . . .

8-3 Case 1: Violation power based consistency measure $r_{p_{v}}$ for the eight One Point Contact models (upper plot). Best (i.e. minimum $r_{p_{v}}$ ) One Point Contact Model(lower plot). . . . . . . . . . . . . . 135 
8-4 Case 2 Experiment: Edge 5 of the vehicle was brought into contact with a fixed cylindrical obstacle. The vehicle was then moved such that it was in sliding contact, where the vehicle motion combined both rotation and translation of the vehicle relative to the contact point. The obstacle was a $12.7 \mathrm{~mm}$ diameter stainless steel bar. . . . . . . . 137

8-5 Case 2: Violation power based consistency measure $r_{p_{v}}$ for the eight One Point Contact models (upper plot). Best (i.e. minimum $r_{p_{v}}$ ) One Point Contact Model(lower plot). . . . . . . . . . . . . . . . . 137

8-6 Case 3 Pure Translation: Edge 5 of the vehicle was brought into full contact with a flat wall.. This contact was maintained throughout the trial as the vehicle was translated first upward and then downward back to its approximate initial position. The wall was smooth, rectangular block of 6061 aluminum. . . . . . . . . . . . . . .

8-7 Case 3: Violation power based consistency measure $r_{p_{v}}$ for the twentyeight Two Point Contact models (upper plot). Best (i.e. minimum $\left.r_{p_{v}}\right)$ Two Point Contact Model(lower plot). . . . . . . . . . . . . . 139

8-8 Case 4 Experiment: The vehicle was brought into contact with two fixed cylindrical obstacles and then rotated in a clockwise direction. Initially vehicle edges 3 and 5 contacted the cylinders( we shall refer to this contact configuration as simply $(3,5))$. As the vehicle rotates, the actual contact state eventually transitions from from $(3,5)$ to $(3,4)$. Both obstacles were $12.7 \mathrm{~mm}$ diameter stainless steel bars. . . . . .

8-9 Case 4: Violation power based consistency measure $r_{p_{v}}$ for the twentyeight Two Point Contact models (upper plot). Best (i.e. minimum $\left.r_{p_{v}}\right)$ Two Point Contact Model(lower plot). . . . . . . . . . . . . . . . 141

8-10 Case 1: Violation energy measure $r_{e_{v}}$ the best One Point Contact model and the best Two Point Contact model (upper plot). Number of contact points associated with the best overall model (lower plot). 
8-11 Case 1: Best Overall Model vs. Time. . . . . . . . . . . . . . . . . . 143

8-12 Case 2: Violation energy measure $r_{e_{v}}$ the best One Point Contact model and the best Two Point Contact model (upper plot). Number of contact points associated with the best overall model. (lower plot). 145

8-13 Case 2: Best Overall Model vs. Time. . . . . . . . . . . . . . 145

8-14 Case 3: Violation energy measure $r_{e_{v}}$ the best One Point Contact model and the best Two Point Contact model (upper plot). Number of contact points associated with the best overall model. (lower plot). 147

8-15 Case 3: Best Overall Model vs. Time. . . . . . . . . . . . . . . . . . . 147

8-16 Case 3: Estimated coefficient of dynamic friction $\mu_{d}$ during Case 3. $\mu_{d}$ was estimated by dividing the contact force's tangential component by its normal component and taking the absolute value. At time $t=0$ no tangential load is applied to the vehicle and its tangential velocity is still zero. At about time $t=2.7$ seconds the vehicle velocity is reversed. The tangential force briefly goes to zero during the reversal, leading to a second dip in the estimated value of $\mu_{d} \ldots \ldots$. . . . . 148

8-17 Case 4: Violation energy measure $r_{e_{v}}$ the best One Point Contact model and the best Two Point Contact model (upper plot). Number of contact points associated with the best overall model. (lower plot). 148

8-18 Case 4: Best Overall Model vs. Time. . . . . . . . . . . . . . 150

8-19 Case 5 Experiment: The vehicle was brought into contact with a set of four fixed cylindrical obstacles. The vehicle was moved such that the sequence of contact states depicted above took place. All four obstacles were $12.7 \mathrm{~mm}$ diameter stainless steel bars. . . . . . . . . . . . . . . 150

8-20 Case 5: Violation energy measure $r_{e_{v}}$ the best One Point Contact model and the best Two Point Contact model (upper plot). Number of contact points associated with the best overall model. (lower plot). 151

8-21 Case 5: Best Overall Model vs. Time. . . . . . . . . . . . . . . . . . 151 
8-22 Violation power metric $r_{p_{v}}$ for Case 4i.e. for $\ldots \ldots \ldots \ldots$

9-1 Example of a gripper with variable geometry fingers dexterously manipulating a handle. . . . . . . . . . . . . . . 157

A-1 Planar vehicle testbed $\ldots \ldots \ldots \ldots \ldots \ldots$

A-2 Geometry of a cable connecting two pulleys of the same radius . . . 174

A-3 Schematic of model vehicle cable drive mechanism . . . . . . . 176

B-1 Case 1 Experiment: Edge 5 of the vehicle was brought into contact with the corner $\mathrm{c} 1$ of a fixed object in environment. The vehicle was then rotated about this corner such that little or no slip occurred at the contact point. Initial rotation was the counter clockwise direction, then the rotation was reveresed, bringing the vehicle (approximately) back to its original position. The obstacle was a square bar of 6061 aluminum alloy. . . . . . . . . . . . . . . . . . . . . . 192

B-2 Case 1: Best Overall Model vs. Time . . . . . . . . . . . . . . 193

B-3 Case 1: Best Number of Contact Points vs. Time . . . . . . . . . 193

B-4 Case 1: Best One Point Model vs. Time. . . . . . . . . . . . . 194

B-5 Case 1: Best Two Point Model vs. Time. . . . . . . . . . . . . . 194

B-6 Case 1: Measured Force and Moment vs. Time. . . . . . . . . . . . . 195

B-7 Case 1: Measured Velocity vs. Time. . . . . . . . . . . . . . 195

C-1 Case 2 Experiment: Edge 5 of the vehicle was brought into contact with a fixed cylindrical obstacle. The vehicle was then moved such that it was in sliding contact, where the vehicle motion combined both rotation and translation of the vehicle relative to the contact point. The obstacle was a $12.7 \mathrm{~mm}$ diameter stainless steel bar. . . . . . . 197

C-2 Case 2: Best Overall Model vs. Time . . . . . . . . . . . . . . 198

C-3 Case : Best Number of Contact Points vs. Time . . . . . . . 198 
C-4 Case 2: Best One Point Model vs. Time. . . . . . . . . . . . 199

C-5 Case 2: Best Two Point Model vs. Time. . . . . . . . . . . . . 199

C-6 Case 2: Measured Force and Moment vs. Time. . . . . . . . . . 200

C-7 Case 2: Measured Velocity vs. Time. . . . . . . . . . . . . 200

D-1 Case 3 Pure Translation: Edge 5 of the vehicle was brought into full contact with a flat wall.. This contact was maintained throughout the trial as the vehicle was translated first upward and then downward back to its approximate initial position. The wall was smooth, rectangular block of 6061 aluminum . . . . . . . . . . . . . 202

D-2 Case 3: Best Overall Model vs. Time . . . . . . . . . . . . 203

D-3 Case 3: Best Number of Contact Points vs. Time . . . . . . . . 203

D-4 Case 3: Best One Point Model vs. Time. . . . . . . . . . . . 204

D-5 Case 3: Best Two Point Model vs. Time. . . . . . . . . . . . 204

D-6 Case 3: Measured Force and Moment vs. Time. . . . . . . . . 205

D-7 Case 3: Measured Velocity vs. Time. . . . . . . . . . . . . 205

E-1 Case 4 Experiment: The vehicle was brought into contact with two fixed cylindrical obstacles and then rotated in a clockwise direction. Initially vehicle edges 3 and 5 contacted the cylinders( we shall refer to this contact configuration as simply $(3,5))$. As the vehicle rotates, the actual contact state eventually transitions from from $(3,5)$ to $(3,4)$. Both obstacles were $12.7 \mathrm{~mm}$ diameter stainless steel bars. . . . . . 207

E-2 Case 4: Best Overall Model vs. Time . . . . . . . . . . . . . 208

E-3 Case 4: Best Number of Contact Points vs. Time . . . . . . . . 208

E-4 Case 4: Best One Point Model vs. Time. . . . . . . . . . . . . . 209

E-5 Case 4: Best Two Point Model vs. Time. . . . . . . . . . . . . 209

E-6 Case 4: Measured Force and Moment vs. Time. . . . . . . . . . . . 210

E-7 Case 4: Measured Velocity vs. Time. . . . . . . . . . . . . 210 
F-1 Case 5 Experiment: The vehicle was brought into contact with a set of four fixed cylindrical obstacles. The vehicle was moved such that the sequence of contact states depicted above took place. All four obstacles were $12.7 \mathrm{~mm}$ diameter stainless steel bars. . . . . . . . . . . . . . . 212

F-2 Case 5: Best Overall Model vs. Time . . . . . . . . . . . . . . 212

F-3 Case 5: Best One Point Model vs. Time. . . . . . . . . . . . . . 213

F-4 Case 5: Best Number of Contact Points vs. Time . . . . . . . . 213

F-5 Case 5: Best Two Point Model vs. Time. . . . . . . . . . . . 214

F-6 Case 5: Measured Force and Moment vs. Time. . . . . . . . . . . 214

F-7 Case 5: Measured Velocity vs. Time. . . . . . . . . . . 215 


\section{List of Tables}

1.1 Probability of failure for a single attempt and number of attempts required to ensure the aggregate chance of failure is less than $.01 . \quad$.

2.1 Properties for the Handles shown in Figure 2-6. Notes: 1. X indicates that the given D.O.F. has the given property. 2. "Friction" means the given D.O.F. is constrained by friction alone. 3. All capture ranges are given in inches and are estimated for a point at a center of the capture range. 4. - means no good estimate available. 5. arb. means a successful grasp can be obtained for any value of the D.O.F. . . . .

3.1 Assumptions for Part II . . . . . . . . . . . . . . . . . . . . . . 69

5.1 The five classes of possible contact models for a rigid, planar body interacting with fixed rigid, planar bodies in its environment . . . .

8.1 Percentage of time the technique selected 1) the correct number of contact points, 2) the best overall model and 3) the best overall model or an immediately adjacent model. . . . . . . . . . . . . . . 153

A.1 Selected implementation options for the primary system functions. . . 166

A.2 Issues involved in the design of the experimental apparatus . . . . . 166

I.1 Mathematical notation . . . . . . . . . . . . . . . . 223 


\section{Chapter 1}

\section{Introduction}

This thesis addresses the problem of reducing a manipulation system's sensitivity to geometric uncertainty, i.e. uncertainty in the knowledge of the geometry and position/orientation of objects in the environment relative to the manipulator. While geometric uncertainty presents problems in any manipulation environment, it presents particularly severe challenges to the completion of undersea manipulation tasks. Undersea manipulation systems typically consist of a manipulator arm mounted to a free flying underwater vehicle. In general the geometric uncertainty associated with the undersea environment is quite high. We typically have little information about the geometry of naturally occurring objects in the undersea environment. Even the engineered objects that we introduce in the course of our tasks tend to have high geometric uncertainty in that their positions relative to the vehicle and the manipulator arm are generally poorly known. In the following section we qualitatively consider the impact of geometric uncertainty on the productivity of a (teleoperated or autonomous) manipulation system. 


\subsection{Effects of misalignment}

To illustrate the effects of geometric uncertainty consider the task of recovering a tool from the ocean bottom using a manipulator mounted to a free flying vehicle. To simplify the problem let us assume that misalignment between the end effector and the tool occurs only in one direction. Let us further assume that the misalignment in this direction can be modeled as a zero mean Gaussian distribution with standard deviation $\sigma$, i.e. that the actual end effector position is equal to the desired position plus a random misalignment component. We define the tolerance ratio $T$ as

$$
T=\frac{\text { misalignment tolerance }}{\sigma}
$$

where the misalignment tolerance is the maximum misalignment between the end effector and tool that can occur and still yield a successful grasp when the end effector closes.

Knowing $T$ we can use the probability distribution function for the zero mean Gaussian to determine the probability of failure $P_{a}$ for a single attempt to grasp the tool. The probability $P_{n}$ that the tool will not be successfully grasped after $n$ attempts is equal to the chance of failure for a single attempt raised to thet $n^{\prime}$ th power, i.e.

$$
P_{n}=P_{a}^{n}
$$

Using this formula we can determine the number of attempts required to guarantee that the aggregate chance of failure $P_{n}$ is acceptably low. Table 1.1 shows the probability of failure for a single attempt and the corresponding number of attempts required to guarante that the aggregate chance of failure $P_{n} \leq .01$ for three different values of the tolerance ratio $\mathrm{T}$.

We can view the different values of $T$ in two different ways.

- If the task and environmental conditions remain fixed, these numbers show 


\begin{tabular}{|c|c|c|}
\hline$T$ & $P_{a}$ & attempts required to ensure $P_{n} \leq .01$ \\
\hline 2 & .05 & 2 \\
\hline .5 & .62 & 9.5 \\
\hline .1 & .92 & 55 \\
\hline
\end{tabular}

Table 1.1: Probability of failure for a single attempt and number of attempts required to ensure the aggregate chance of failure is less than .01.

that increasing the misalignment tolerance of the system significantly reduces the chance of failure and, concomitantly, the number of attempts required to successfully complete the task.

- If instead the misalignment tolerance of the system remains fixed, these numbers show that whether or not a task can be efficiently performed depends upon the natural misalignment associated with it.

Note that when $T$ is very small the number of attempts required to ensure success will be so large as to render the task effectively impossible.

\subsection{General Problem Statement}

The general objective of this thesis is to develop techniques to maximize the tolerance ratio $T$ for a vehicle mounted subsea manipulation system.

\subsection{Approach}

There are two obvious ways to maximize $T$; one is to maximize the misalignment tolerance of the manipulation system, the other is to minimize the magnitude of the misalignment presented to the manipulation system. This thesis pursues both approaches. 
Part I of this thesis focuses on minimizing the system sensitivity to misalignment through the design of a misalignment tolerant grasping system. Part II of this thesis focuses on minimizing the vehicle's contribution to the misalignment presented to the manipulator by developing the tools necessary to allow the vehicle to exploit contact with fixed objects in the environment to help stabilize the vehicle against manipulation reaction loads. Specifically, we develop a contact identification system which determines the vehicle's points of contact with the environment as well as the constraints these contacts impose on the vehicle motion. Such a system, when integrated with a hybrid controller, enables the vehicle to control its unconstrained degrees of freedom while in contact. 


\section{Part I}

Minimizing System Sensitivity to Misalignment 


\section{Chapter 2}

\section{Devising a Misalignment Tolerant Subsea Grasping System}

\section{$2.1 \quad$ Abstract}

This chapter addresses the problem of devising a subsea grasping system which tolerates significant pre-grasp misalignment between the gripper and its intended task object. We begin by discussing the desired characteristics and the required functions of such a system and use this information to select the most suitable approach for coping with misalignment. We present a 1 degree-of-freedom (D.O.F.) gripper design which is guaranteed, in the absence of external loads, to align cylinders of various diameter despite significant initial misalignment with respect to the gripper body. We then present a handle design which, when grasped by a modified version of the gripper, also aligns with respect to the gripper body but, once grasped, requires no actuator torque to resist a wide range manipulation loads. A suite of compatible, positively locating handles is presented, each imposing a different degree of constraint and each able to resist a different subset of manipulation loads. The chapter concludes with a discussion of successful field testing of the system and a brief consideration of the applicability of this work to the problem of vehicle docking. 


\subsection{Introduction}

In subsea manipulation the presence of ocean currents, incomplete knowledge of work site geometry and limitations on vehicle and manipulator control performance all combine to guarantee imperfect alignment between the manipulator and its intended task object. If the misalignment exceeds the misalignment tolerance of the grasping system, the task object cannot be acquired unless the gripper is repositioned.

In manned submersible and Remotely Operated Vehicle (ROV) systems a human operator controls the manipulator motion. Fitts [11] showed that, for humans moving an object from a given position to a target area, the time required to complete the move decreases steadily as the size of the target area is increased. Thus increasing the robustness to misalignment of grasping systems should improve the productivity of manned submersible and ROV based manipulation systems by reducing the time required to acquire task objects. We expect a more pronounced improvement in productivity for ROVs than for manned systems. In ROV systems multiple monocular video images replace the direct stereoscopic view of the work site available with manned submersible systems. The associated loss of depth perception significantly degrades the human operators ability to compensate for misalignment.

In AUV based manipulation systems the continuous, real-time human intervention that ROVs and manned submersibles depend on for coping with misalignment will be precluded by the limitations of acoustic communication channels ( $10 \mathrm{kbits} / \mathrm{s}$, 10s time delay). Sayers [24] imposed these limitations on a real ROV manipulation system in order to demonstrate the effectiveness of the teleprogramming paradigm for dealing with these limitations. The ROV was actively driven against the sea floor (depth: 7 meters) to minimize wave induced motion of the vehicle. Due to the grippers sensitivity to misalignment, however, the small vehicle motions which still occurred drastically hindered or even prevented successful completion of most tasks. Replacing this gripper with a misalignment tolerant gripper (the system presented in Section 2.5 of this chapter) dramatically improved the task completion success rate. 
Based on this experience, we believe that misalignment tolerant manipulation systems will be absolutely essential to performing even basic AUV based manipulation tasks.

A large body of work exists on general purpose grippers. Skinner [26] constructed a three fingered gripper that could reproduce most of the basic human grasp modes. Rovetta [20] developed a three fingered gripper capable of limited adaption to an arbitrary object geometry. Salisbury introduced a fully actuated three fingered cable driven gripper capable of dexterously manipulating grasped objects through finger motion alone. Jacobsen [15] developed a four fingered tendon driven hand comprised of three multi-degree-of-freedom fingers and an articulated thumb. Ulrich [28] constructed a modified version of the Skinner gripper which added an additional grasp mode and explicitly made use of contact with the gripper's "palm" to facilitate the use of power grasps. Most recently, Lane [16] developed a gripper for undersea use comprised of three hydraullically actuated tentacle-like fingers.

Although each of these grippers can successfully grasp a wide range of object shapes, only the dexterous grippers built by Salisbury, Jacobsen and Lane address the issue of specifically orienting a grasped object relative to the manipulator. These grippers (as is the case for the rest of the grippers cited) generate relatively low grasp forces and rely primarily on friction forces to constrain grasped objects. This renders these grippers unsuitable for tasks requiring accurate positioning of heavy objects (e.g. water samplers, sediment samplers) and/or those involving high manipulation loads (e.g. mating connectors, collecting geological samples).

Tool changer mechanisms (i.e. mechanisms which permit the rapid and automatic interchange of different tools to a robots endpoint) represent the opposite extreme in grasping system design. These mechanisms are designed to "grasp" only one specialized shape, copies of which are attached to the objects to be grasped. Tool changers have been developed for industrial, space [31] and subsea [13] environments. While these mechanisms tolerate high forces and are mechanically robust, they tolerate only small $(+/-.6 \mathrm{~cm}$. $)$ misalignment and cannot be conveniently scaled up to the $+/-2$ 
to $5 \mathrm{~cm}$. desired for subsea systems. Furthermore, such systems obviously cannot be directly used to grasp unstructured objects (e.g. rocks, biological samples).

An intermediate approach is to design an easily grasped object (i.e. a handle) for use with a modestly general purpose gripper. This is the approach pursued in this thesis. For our purposes the gripper/handle system should be inherently tolerant of pre-grasp misalignment and should efficiently resist manipulation loads. A good example of a system with these characteristics is the self aligning finger/handle system developed by Voellmer [30] for parallel jaw grippers. Although this system could be adapted for subsea use, we believe that the gripper/handle solution proposed in the following sections better satisfies the needs of subsea grasping systems.

A closely related body of work has been done by Brost and Goldberg [6], Schimmels and Peshkin [25], Bausch and Youcef-Toumi [4], and Asada [2] on the synthesis of optimal pin fixtures for the constraint and alignment (given small initial misalignment) of parts of known geometry in a plane. The work proposed here considers a related problem: the synthesis of a moving part geometry (i.e. a set of fingers) which, when brought into contact with a significantly misaligned rigid assembly of pins, aligns and efficiently constrains the assembly with respect to the base to which the fingers are attached.

The goal of this chapter is the development of a misalignment tolerant gripper/handle system suitable for use with manned submersible, ROV and AUV based manipulation systems. In Section 2.3 we discuss how misalignment impacts grasping and suggest a set of desirable system characteristics. Section 2.4 discusses the general requirements for ensuring proper alignment between the handle and gripper and the requirements for efficiently resisting manipulation loads. Section 2.5 presents a gripper which is shown to accurately and repeatably locate cylinders of various radii. Section 2.6 discusses a modified version of this gripper and introduces a handle design which, when grasped by the modified gripper, repeatably locates and requires no actuator torque to resist most manipulation loads. Finally, Section 2.7 presents addi- 
tional positively locating/load resisting handles compatible with the proposed gripper designs while Section 2.8 discusses successful field use of the completed system.

\subsection{Grasping and misalignment}

The purpose of a grasping system is to perform the following three functions which we define as

1. Capture, i.e. acquire control of an object's position and orientation.

2. Contain, i.e. control an object's position/orientation despite the presence of manipulation and disturbance loads

3. Release, i.e. disengage from an acceptably repositioned object (This implies that the act of disengaging should not perturb the object from its new position/orientation.).

We assume that at each stage there exists some desired relative position/orientation between the gripper and the task object and define misalignment to be the difference between this desired state and the actual state. We can therefore identify a different type of misalignment for each of the aforementioned functions, these being

1. Pre-capture misalignment - the difference between the object's position in relation to the gripper when grasping begins and the position it would occupy within the gripper if successfully grasped.

2. Post-capture misalignment - the difference between an objects perceived and actual position/orientation with respect to the gripper while grasped.

3. Post-release misalignment - the difference between the objects intended and actual position/orientation with respect to the environment after it has been released. 
For a given gripper and object, pre-capture misalignment determines if and in what mode an object will be grasped. Post-capture misalignment determines how accurately the objects position can be specified while grasped and post-release misalignment determines how accurately an object can be repositioned with respect to the environment. Thus the ideal gripper is tolerant of substantial pre-grasp misalignment but has zero post-capture and post-release misalignment.

A successful grasping system, however, must do more than just cope with misalignment. We offer the following as a reasonable, partial set of characteristics for a science/servicing oriented subsea grasping system.

1. highly tolerant of pre-capture misalignment $(+/-1$ to $2 \mathrm{in}$. desired)

2. near zero post-capture misalignment

3. capable of high accuracy positioning of structured objects (e.g. tools, samplers, connectors) despite large manipulation loads

4. capable of low accuracy positioning of a range of unstructured objects (e.g. rocks, mussels, tube worms)

5. capable of low force grasping of fragile objects (e.g. biological samples, archeological artifacts)

6. robust to corrosion, extreme pressure, thermal expansion, impact loads and operation in the presence of sand and sediment particles).

\subsection{Issues concerning misalignment tolerant grip- per/handle systems}

Positive location of the handle: Since the handle and gripper are initially misaligned, positive location requires that the handle and/or the gripper move. Assume for the 
moment that only the handle shifts position. To properly align the contact forces between the gripper and handle during capture must induce relative motion which drives the handle to the desired relative position. Three types of forces will act on the handle; contact normal forces $f_{N}$, contact friction forces $f_{f r}$ and constraint forces $f_{c}$ acting on the object attached to the handle. Positively locating the handle requires, coarsely speaking, that $f_{N}>f_{f r}+f_{c}$ and that the induced motion move the handle towards its desired alignment (ideally the desired alignment is the only possible grasp mode for the handle). Therefore, we must select a finger and handle geometry which produces the desired forces and must provide the gripper with enough actuation force to ensure that the desired motion occurs.

Efficiently resisting manipulation loads: Having aligned the handle the gripper's function becomes containment, i.e. maintaining alignment despite the application of large manipulation loads. The gripper/handle system effectively "maps" the $\Re(6)$ vector space of handle loads into the $\Re(m)$ vector space of actuator torques (where $m$ is the number of actuators in the gripper). From linear algebra, therefore, we conclude that it is possible to resist up to a $\Re(6-p)$ vector space of applied forces with no actuator torque where $p \leq m$ is the number of non-redundant actuators in the gripper. Such applied forces lie in the null space of the actuator space and represent loads which are entirely borne by the structure of the gripper. We refer to these loads as actuator-orthogonal loads. Assuming finite actuator output, we can maximize a gripper's ability to resist manipulation loads by minimizing the number of actuators used and by selecting a finger/handle geometry which maximizes the dimension of the actuator orthogonal load space.

\subsection{Mislignment Tolerant Grasping of Cylinders}

Figure 2-1 shows a 1 DOF gripper design which, as we will show, positively locates cylinders despite significant pre-grasp misalignment. To show this we first find the 


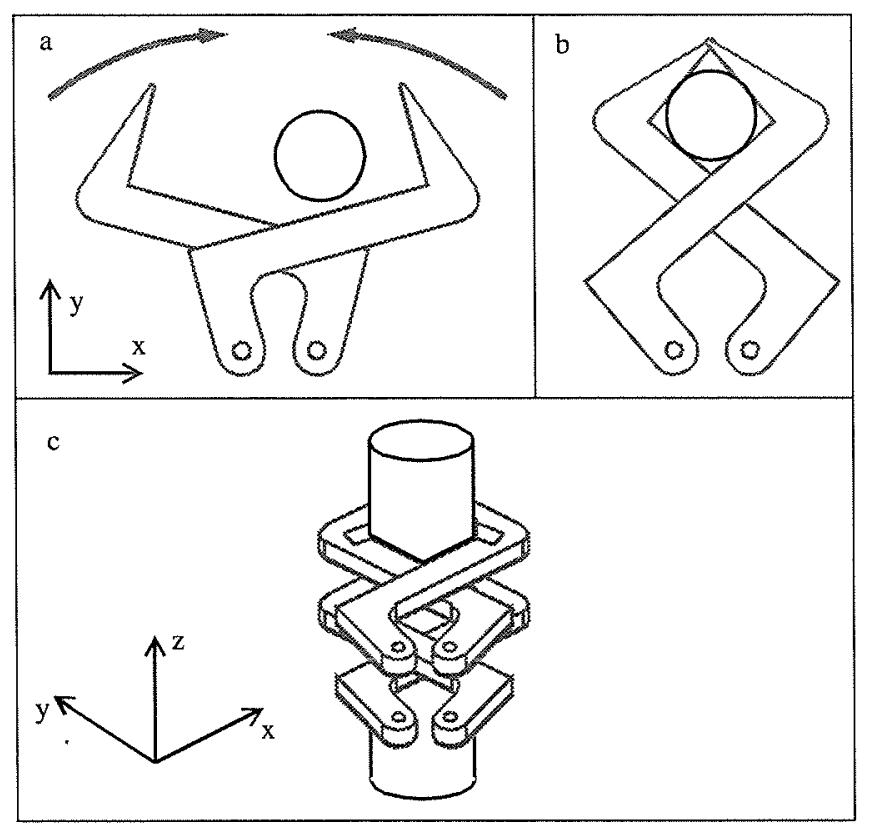

Figure 2-1: 1 DOF gripper which positively locates grasped cylinders: a. during capture, plan view. b. cylinder fully grasped, plan view. c. cylinder fully grasped, isometric view showing that each of the grippers "fingers" actually consists of two plates separated in the z-direction.

cylinders' location when fully grasped and then determine the conditions required for the cylinder to reach this position during grasping.

Location when fully grasped: Due to the symmetric motion of the fingers (they are linked so that they always rotate through equal but opposite angles) the cylinder's final position $C_{f}$ lies a distance $H_{g}$ directly above the midpoint between the two finger pivot points (see Figure 2-2). To bring the cylinder to this position each finger must rotate through an angle of $\theta_{g}$. Solving for $\theta_{g}$ and height $H_{g}$ we obtain

$$
\begin{gathered}
\theta_{g}=\arccos \left(\frac{D}{2 R}\right)-\gamma \\
H_{g}=R \sin \left(\theta_{g}+\gamma\right)
\end{gathered}
$$


where $D$ is the distance between the finger pivot axes, $R$ is the length of the line drawn between a finger's axis and the cylinder axis when the cylinder is firmly seated against the internal corner of the finger (as shown in Figure 2-2), and $\gamma$ is the angle between this line and a line drawn between the finger pivot axes.

Alignment force during capture: To prove that the cylinder reaches this position we look at the forces acting on the system while the gripper is closing. Initially only one finger will contact the cylinder. If the closing torque is large enough to overcome the external forces acting on the cylinder, the cylinder will be pushed towards the centerline of the gripper. During this motion the cylinder will also slide and/or roll along the finger surface, resulting in two possible grasp scenarios. In the first the cylinder contacts the finger's outer end before full closure. In this case motion in relation to the finger ceases and the cylinder travels directly to the final location given by equations 2.1 and 2.2 along a circular arc of radius $R$. Successful grasping in this scenario requires only that the closure torque be great enough to overcome the external loads acting on the cylinder.

In the second scenario the cylinder comes into contact with both fingers prior to full closure (see Figure 2-3a ). Forcing the cylinder to its final location now requires that net outward force applied by the fingers exceeds the sum of the external loads and the friction forces associated with the contact forces. To gauge the grippers ability to do this we will examine the outward force $F$ it can apply to the cylinder.

Recalling that the fingers are linked by a 1:1 gear train we find that the relationship between the closure torque and the contact normal forces $N$ in Figure 2-3a. and b. is

$$
M=2 N s+2 \mu_{s} N h
$$

where

$$
s=s_{1}+s_{2}=(h+r) \tan \theta+\frac{D}{2 \cos \theta}
$$




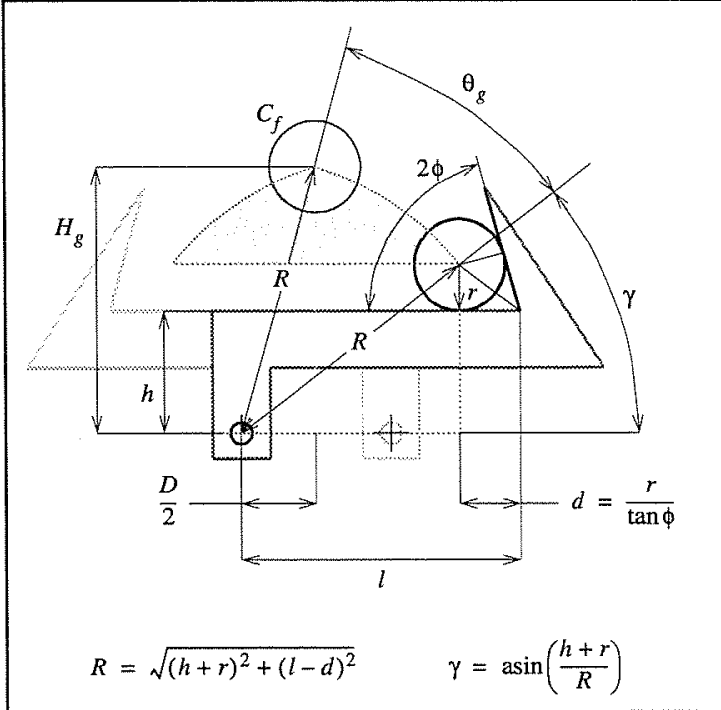

Figure 2-2: Grasping geometry showing the cylinder's final position and the cylinder's capture range (shaded area). Successful grasping is guaranteed if the cylinder's center lies in this region and the external loads meet the conditions discussed in the text.

and $\mu_{s}$ is the static coefficient of friction between the fingers and the cylinder. Balancing the forces acting on the cylinder (Figure 2-3b.) we find that the cylinder will not move unless

$$
F<2 N \cos \theta\left(1-\mu_{s} \tan \theta\right)
$$

At equilibrium we may write equation 2.5 as an equality. Using equations 2.3 and 2.4 to eliminate $N$ and $s$, we find that the relationship between the outward force $F$ resulting from a closure torque $M$ can be written in dimensionless form as

$$
\frac{F D}{M}=\frac{(1-\mu \tan \theta) \cos ^{2} \theta}{\left(\frac{h}{D}+\frac{r}{D}\right) \sin \theta+\frac{1}{2}+\mu \frac{h}{D} \cos \theta}
$$

To show that a given version of the gripper positively locates cylinders we must show that the dimensionless outward force given by equation 2.6 is greater than zero for any closure angle $\theta_{g}$ encountered during the grasp. 


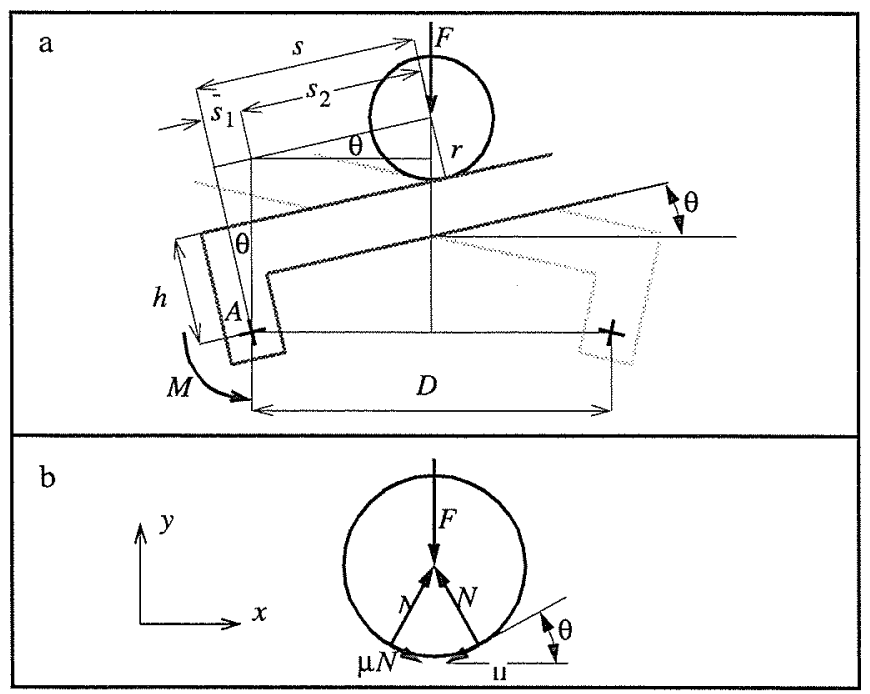

Figure 2-3: Forces acting on a cylinder in contact with both fingers as the gripper closes. a. Geometry and external forces (i.e. finger closure torque $M$ and a purely vertical external force acting at the cylinder center $F$ ) b. detail of frictional, normal and external loads acting on the cylinder ( $\theta$ is the angle between a line connecting the contacting surface of the finger).

The outward force $F$ decreases as the closure angle $\theta_{g}$ increases. To see this consider the slip condition (equation 2.5 ) when $F=0$ (i.e. when there is no external load on the cylinder).Rewriting equation 2.5 for this case we find that the cylinder will not move unless

$$
|\tan \theta|<\frac{1}{\mu} \text { or } \cos \theta>0
$$

The degree to which these conditions are satisfied is maximized when $\theta=0$ (i.e. when the fingers are fully open) and decreases steadily as $\theta$ increases. This implies that the minimum outward force occurs at the maximum closure angle encountered in the grasp, i.e. that we should replace $\theta_{g}$ by the final closure angle in equation 2.6 to verify that $F$ is always positive during a given grasp.

We may now determine the worst case (i.e. minimum) outward force $F$ generated 
by the gripper during the grasp. Figure 2-4 shows the dimensionless version of $F$ as a function of the dimensionless cylinder radius and various values of $\mu_{s}$ for the proposed

design (i.e. $\frac{h}{D}=1.5, \frac{l}{D}=3.05, \phi=\frac{\pi}{2}$ ) The given design is seen to be capable of positively locating any cylinder whose radius is below the maximum graspable radius $\left(\frac{r}{D} \approx 1.5\right)$ as long as $\mu_{s}<0.7$ and the applied dimensionless load does not exceed the associated value given in Figure 2-4. Note, however, that the ability to positively locate a cylinder decreases as the size of the cylinder decreases and as the magnitude of $\mu_{s}$ increases.

Capture range: By the above arguments one can deduce that positive location of a cylinder is guaranteed if its axis lies within the shaded area shown in Figure 2-2. For the proposed design the width of this region's base is $2 l-D-2 r$. For example, if $r=1.0 \mathrm{in}$. the width of the capture region's base is $6.27 \mathrm{in}$. The curves defining the region's upper bounds are circular arcs of radius $R$ centered about each finger's pivot point. Thus a cylinder's capture range increases as we decrease its radius but, as shown in the preceding section, its ability to be positively located decreases.

Ability to resist applied loads: The grasped cylinder is not well suited to resisting manipulation loads. For instance, constraining the cylinder's rotation about or translation along its axis depends entirely on frictional forces. In the next section we present a modified gripper and handle design which avoids these drawbacks but retains the desirable feature of positive location of the handle.

\subsection{Expanding the actuator-orthogonal load space}

Figure 2-5 shows a modified finger design grasping a handle made from two cylinders attached by a rod. We now consider the properties of this system.

Ability to resist applied loads: Assuming zero clearance between the cylinders and the notches, the handle requires no actuator torque to resist $\mathrm{z}$-axis moment or $\mathrm{y}$ direction forces when fully grasped by the modified gripper. To see this note that each 


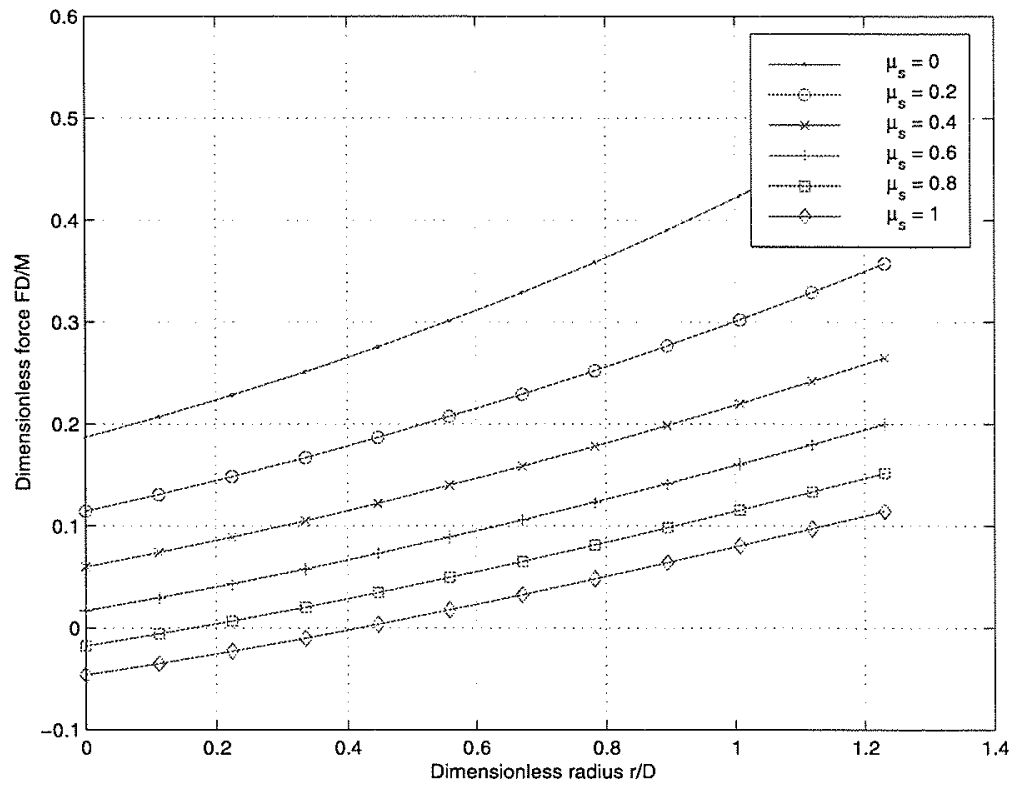

Figure 2-4: The minimum dimensionless (quasi-static) outward force applied to a cylinder during closure of the gripper as a function of the dimensionless radius of the cylinder to be grasped and the associated static coefficient of friction. Combinations for which $\frac{F D}{M}>0$ are guaranteed, in the absence of external forces, to reach the desired final position/orientation. Combinations for which $\frac{F D}{M} \leq 0$ indicate that the cylinder reaches static equilibrium (i.e. jams) prior to reaching the desired position/orientation. 
finger notch is comprised of circular arcs centered on the given finger's axis of rotation. (see Figure 2-2a). As such the line of action of any contact forces associated with these arc surfaces passes through the finger's axis of rotation and therefore induces no torque about the finger axis.

In reality, however, there is clearance between the cylinders and the notches. Nonetheless, the handle is held with no play. The contact forces associated with the ends of the notches (see Figure 2-5) force the handle against the outer arc $R_{o}$ of the notches. Since these contact forces do not pass through the finger pivot points, forces applied to the handle tend to wedge the gripper open. For example, a large enough load in the negative y-direction will wedge the gripper open. The handle will move downward slightly but stops when it contacts the notch's inner surface $R_{i}$ because the contact forces once again pass through the finger's axes of rotation. Thus the handle is positively located for loads less than a certain threshold (defined by the maximum closure torque). For larger loads the handle shifts very slightly but remains solidly grasped.

Location when fully grasped: Employing arguments similar to those used for the cylinder it can be shown this handle positively locates in both the original and modified grippers. When fully grasped by the modified gripper the handle is centered between the two fingers at a height of $H=\sqrt{\left(R_{o}-r\right)^{2}-\left(\frac{l_{h}}{2}\right)^{2}}$ (see Figure 2-5b).

Release of the handle: Because the notches are circular arcs, opening the gripper causes it to disengage from the handle without perturbing the handle's position.

\subsection{Additional positively locating handles}

The modified gripper design is compatible with a wide range of handle designs, each possessing different alignment and load resisting properties. Figure 2-6 shows four handles which positively locate to different degrees when grasped. The X-handles positively locate in relation to the gripper in all 6 D.O.F., the H-handle positively 


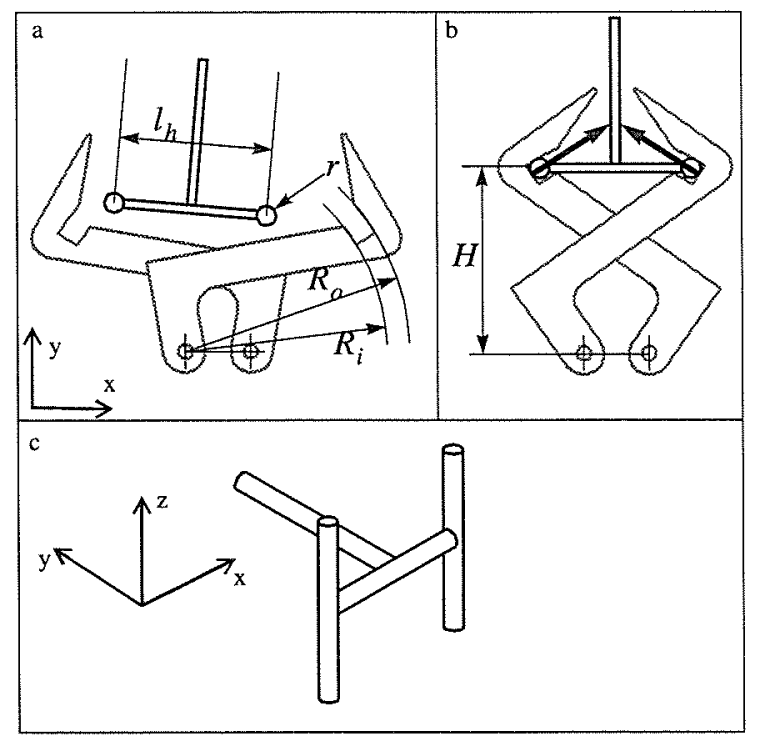

Figure 2-5: Modified gripper design a. H-handle during capture b. H-handle fully grasped, plan view. c. H-handle, isometric view

locates in 5 D.O.F. and the cylinder positively locate in four D.O.F. In general, handles which constrain more degrees of freedom are more sensitive to misalignment, e.g. the X-handles can only be grasped for a limited range of $\theta_{z}$ values while the cylinder can be successfully grasped for any value of $\theta_{z}$. By selecting an appropriate handle and properly speciying its dimensions we can tailor the characteristics of a handle to match the specific needs of a given task, thereby enhancing manipulation efficiency. (Note: several other positively locating handle designs have been omitted for the sake of brevity).

Table 2.1 summarizes the properties of these handles and gives very rough estimates for acceptable misalignment for each handle in the various directions. The actual capture range for each handle is a complicated function of the handle dimensions and its relative position/orientation in relation to the gripper. The values given in the table are estimates of the tolerable misalignment in each D.O.F. when the handle is nominally centered in the actual capture range 


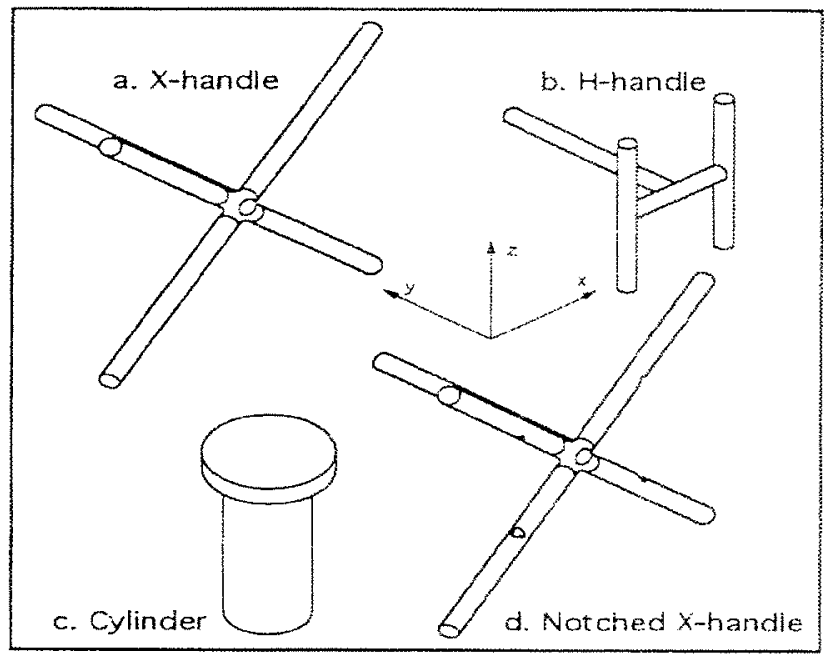

Figure 2-6: Additional handles which positively locate in a. 6 D.O.F., b. 5 D.O.F., c. 4 D.O.F. and d. 6 D.O.F..

\section{$2.8 \quad$ Field testing}

A subsea grasping system embodying these concepts has been constructed and integrated into the manipulation subsystem of the Jason remotely operated vehicle (ROV). The Jason vehicle is an unmanned, tethered system which is teleoperated from a surface support ship. The grasping system was first used during voyage leg KN 145-19 of the research vessel R/V Knorr operated by the Woods Hole Oceanographic Institution. This was a 6 week research cruise organized by Chief Scientist Dr. Daniel J. Fornari to study the mid-Atlantic ridge (depth:1700 m) in the summer of 1996. The main objective of the manipulation portion of this cruise was the collection of water samples, geological samples and biological samples from hydrothermal vents located in the Lucky Strike area of the ridge. Hydrothermal vents are essentially deep sea hot water geysers spewing turbulent plumes of super-heated ( $300 \mathrm{C}$ ) sea water into the ambient $4 \mathrm{C}$ ocean bottom water

Figure 2-7 shows a vehicle-eye view of a fully loaded elevator platform used to transfer equipment and samples between the surface and the ocean floor. Prior to the 


\begin{tabular}{|c|c|c|c|c|c|c|}
\hline Handle & $\mathrm{X}$ & $\mathrm{y}$ & $\mathrm{z}$ & $\theta_{x}$ & $\theta_{y}$ & $\theta_{z}$ \\
\hline \multirow{3}{*}{ Notched X } & $\mathrm{X}$ & $\mathrm{X}$ & $\mathrm{X}$ & $\mathrm{X}$ & $\mathrm{X}$ & $\mathrm{X}$ \\
\cline { 2 - 7 } & & $\mathrm{X}$ & $\mathrm{X}$ & $\mathrm{X}$ & $\mathrm{X}$ & $\mathrm{X}$ \\
\cline { 2 - 7 } & & & & & & \\
\cline { 2 - 7 } $\mathrm{X}$ & \pm 1.5 & \pm 1.2 & \pm 2.0 & - & $\pm 25 \operatorname{deg}$ & - \\
\hline & $\mathrm{X}$ & $\mathrm{X}$ & $\mathrm{X}$ & $\mathrm{X}$ & $\mathrm{X}$ & $\mathrm{X}$ \\
\cline { 2 - 7 } & & $\mathrm{X}$ & & $\mathrm{X}$ & & $\mathrm{X}$ \\
\cline { 2 - 7 } & \pm 1.5 & \pm 1.2 & \pm 2.0 & - & $\pm 25 \operatorname{deg}$ & - \\
\hline \multirow{4}{*}{$\mathrm{H}$} & $\mathrm{X}$ & $\mathrm{X}$ & & $\mathrm{X}$ & $\mathrm{X}$ & $\mathrm{X}$ \\
\cline { 2 - 7 } & & $\mathrm{X}$ & & $\mathrm{X}$ & $\mathrm{X}$ & $\mathrm{X}$ \\
\cline { 2 - 7 } & & & $\mathrm{X}$ & & & \\
\hline \multirow{3}{*}{ Cylinder } & \pm 1.5 & \pm 1.2 & arb. & - & $\pm 25 \operatorname{deg}$ & - \\
\cline { 2 - 7 } & $\mathrm{X}$ & $\mathrm{X}$ & & $\mathrm{X}$ & $\mathrm{X}$ & \\
\cline { 2 - 7 } & & & & & & $\mathrm{X}$ \\
\cline { 2 - 7 } & \pm 2.1 & \pm 0.8 & arb. & $\pm 23 \mathrm{deg}$ & \pm 40 deg. & arb. \\
\hline
\end{tabular}

Table 2.1: Properties for the Handles shown in Figure 2-6. Notes: 1. X indicates that the given D.O.F. has the given property. 2. "Friction" means the given D.O.F. is constrained by friction alone. 3. All capture ranges are given in inches and are estimated for a point at a center of the capture range. 4. - means no good estimate available. 5. arb. means a successful grasp can be obtained for any value of the D.O.F. 
use of elevator platforms vehicle mission lengths were limited by how much equipment the vehicle could carry and all material transfer between the work site and the surface required recovery of the vehicle. Using free falling/free ascending elevator platforms enables the vehicle to remain on-site and work continuously. The success of this approach depends strongly on the manipulator's ability to efficiently grasp objects since the manipulator must transfer all equipment and samples between the platform and the vehicle.

Two of the tasks required sampling of unstructured objects. Figure 2-8 shows the gripper grasping a sulfide rock sample recovered from the ocean floor. Other rock samples were collected by grasping outcroppings on vent mounds and breaking them free. Over one hundred pounds of rock samples were collected during the cruise. Figure 2-9 shows the manipulator collecting a clump of vent dwelling mussels. Both types of samples were deposited into hinged top bio-boxes which were subsequently transferred back to the elevator platforms.

The remaining tasks largely involved interaction with structured objects. In Figure 2-10 the manipulator takes a sediment sample by plunging a core tube sampler into the bottom (returning the sampler to its sleeve fit holster prevents the sediment from dislodging from the tube during recovery). This sampler exemplifies the idea of matching the handle to the task. Being axially symmetric, the quality of the sediment sample is independent of the orientation of the sampler about this axis. This permits the use of a cylinder type handle which is more compact and misalignment tolerant than either the $\mathrm{H}$ or $\mathrm{X}$ type handles.

In Figure 2-11 the operator uses the shaft of an X-type handle as a hammer and prybar to break rock away from a vent to enlarge the orifice of a plume in preparation for sampling of the plume water. The X-type handle best addresses the need to resist the associated forces (i.e. large magnitudes, arbitrary directions). Video from the task site shows that the X-handle remained firmly grasped without shifting throughout this procedure. 
Finally, Figure 2-12 shows the gripper grasping a vent water sampler. Sampling vent water was probably the most demanding grasping task performed during the cruise because it requires that the sampler be precisely positioned with respect to the gripper despite the application of large forces to trigger the sampler.

Vent water sampling proceeds as follows. When the operator grasps a water sampler electromagnetic coils in the gripper and the sampler are brought into alignment, enabling the inductive communication of water temperature data from a sampler to the vehicle. Having grasped a sampler, the operator positions its sampling tube in the enlarged plume orifice, locates the hottest portion of the plume and then triggers the spring-loaded sampling bottle to take a sample

Triggering the sampler requires the gripper to resist a force of 50 to $75 \mathrm{lbf}$. and a moment of 175 to 260 in.-lbf. torque. Any shifting of the handle in relation to the gripper can ruin the sample either by breaking the inductive link (causing the loss of temperature data) or by changing the position of the samplers inlet tubes (the ends of the sampler tubes extend two feet in front of the plane of the handle. In addition, the water temperature varies dramatically with position within the plume due to the violent turbulent mixing taking place between the superheated plume water and the near zero ambient sea water). The positive location and actuator orthogonal load characteristics of the X-handle enabled the collection of continuous temperature data throughout the triggering and sampling process with no perceptible motion of the sampler inlet tube.

Since the initial field deployment the grasping system has been used for a wide variety of additional ocean science tasks at depths exceeding 5000 meters. These tasks the include mating and unmating of undersea electrical connectors during the installation and testing of the Hawaii Ocean Observatory, excavating sediment and precisely placing seismometers on the ocean floor, and the recovery of archeological artifacts from ancient ship wrecks in the abyssal plane of the Mediterranean. 


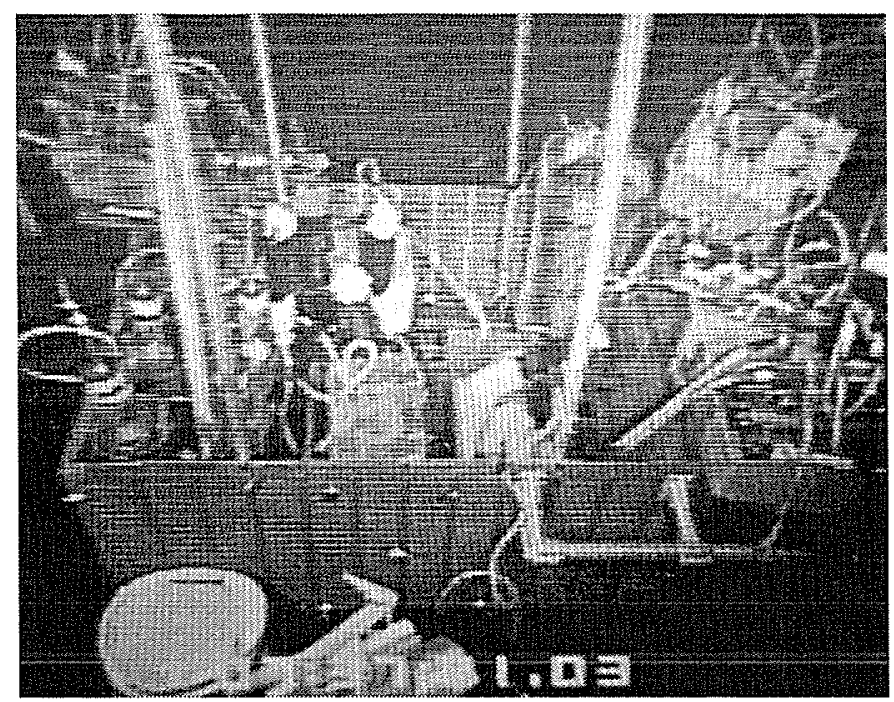

Figure 2-7: Elevator platform loaded with core tubes, water samplers and bio-boxes. Elevator platforms transfer tools and samples between the surface and the work site, enabling the vehicle to remain on site indefinitely. The vehicle operator uses the manipulator to transfer all material between the vehicle and the elevator

\subsection{Conclusions}

Misalignment between a robots gripper and its task object cannot be avoided in subsea manipulation. Significant misalignment tolerance is essential to the efficient performance of manipulation tasks. The grasping system presented in this paper represents an attempt to address the grasping needs of manned submersible, ROV and AUV based manipulation systems. The system is robust to misalignment and manipulation loads and offers substantial flexibility in adapting to the needs of different tasks. Finally, the concepts presented here apply equally well to the problem of docking a vehicle to a separate structure. A scaled version of the grasping mechanism could serve as a flexible, simple and precise vehicle docking mechanism. 


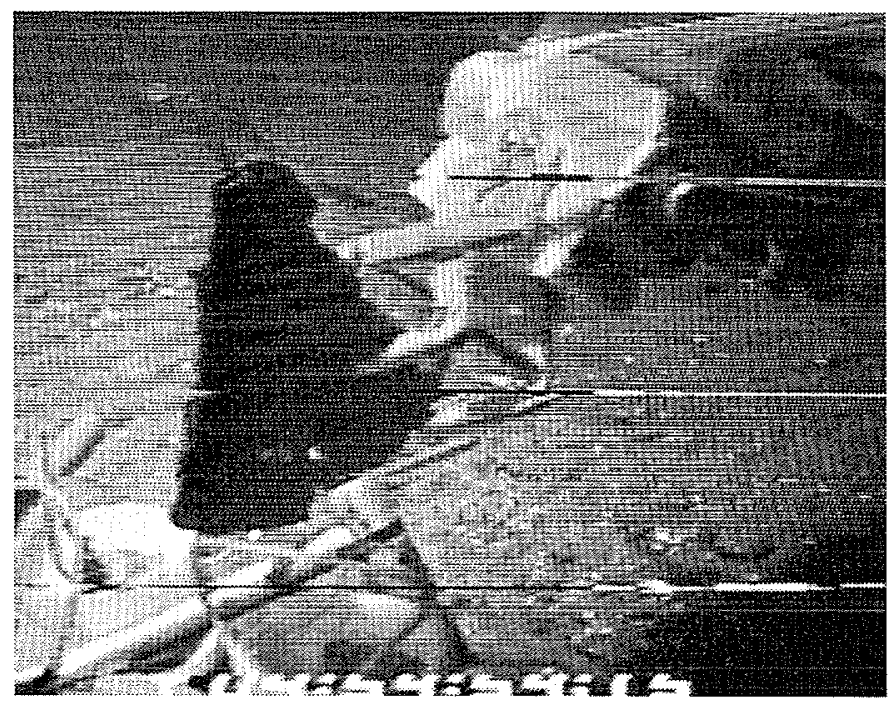

Figure 2-8: Gripper grasping sulfide rock sample. The operator uses the gripper to break samples off from the vent structure or to pick up samples lying on the bottom

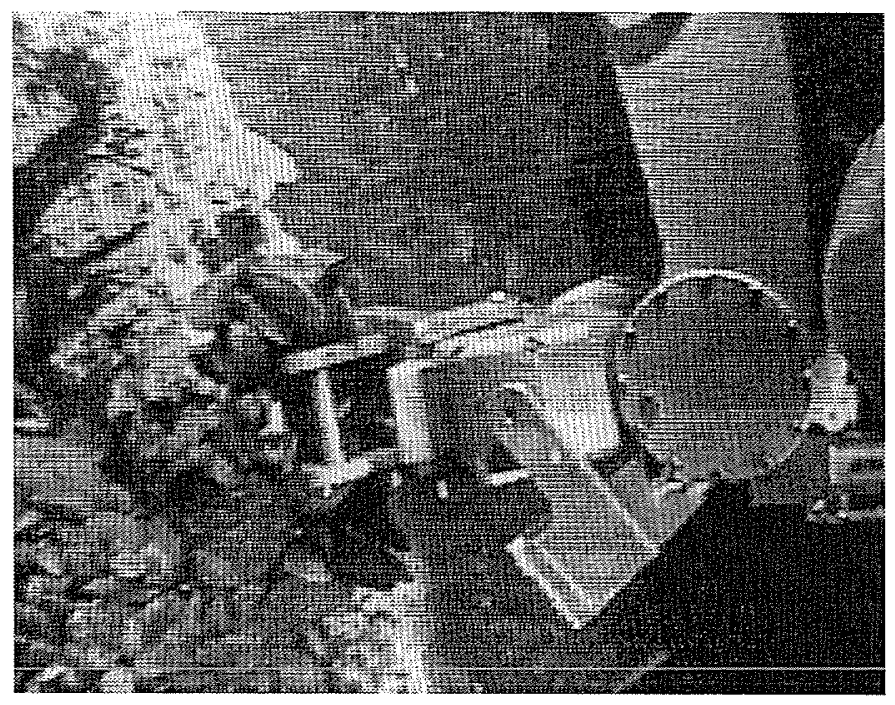

Figure 2-9: Gripper collecting mussel samples. Mussel samples are subsequently placed in a bio-box on the vehicle and eventually the full bio-box is transferred back to the elevator platform and replaced with an empty bio-box. 


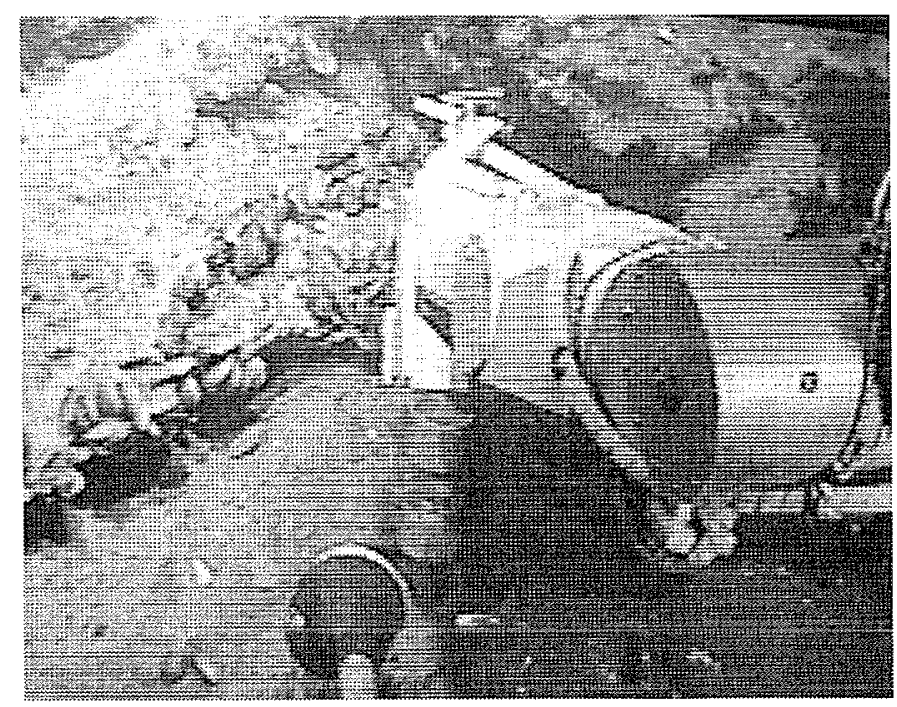

Figure 2-10: Gripper grasping core tube sampler. Plunging the sampler into the sediment collects a cylindrical sample of bottom material. The full core tube is placed into a sleeve fit holster for eventual return to an elevator platform. (Outline added by authors.).

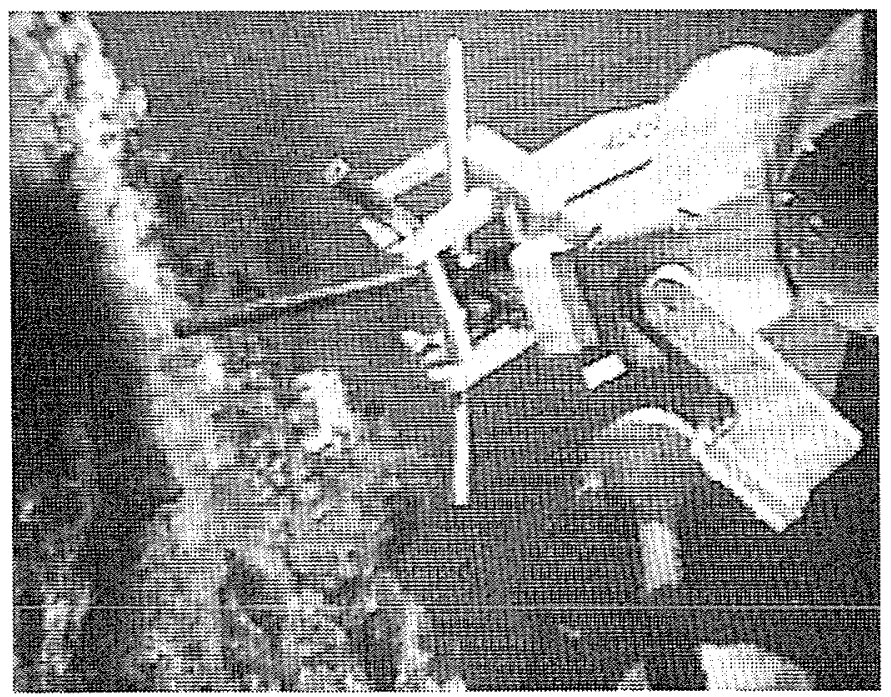

Figure 2-11: Gripper using an X-handle to enlarge plume orifice. Vent orifices are enlarged to facilitate sampling of the plume water. 


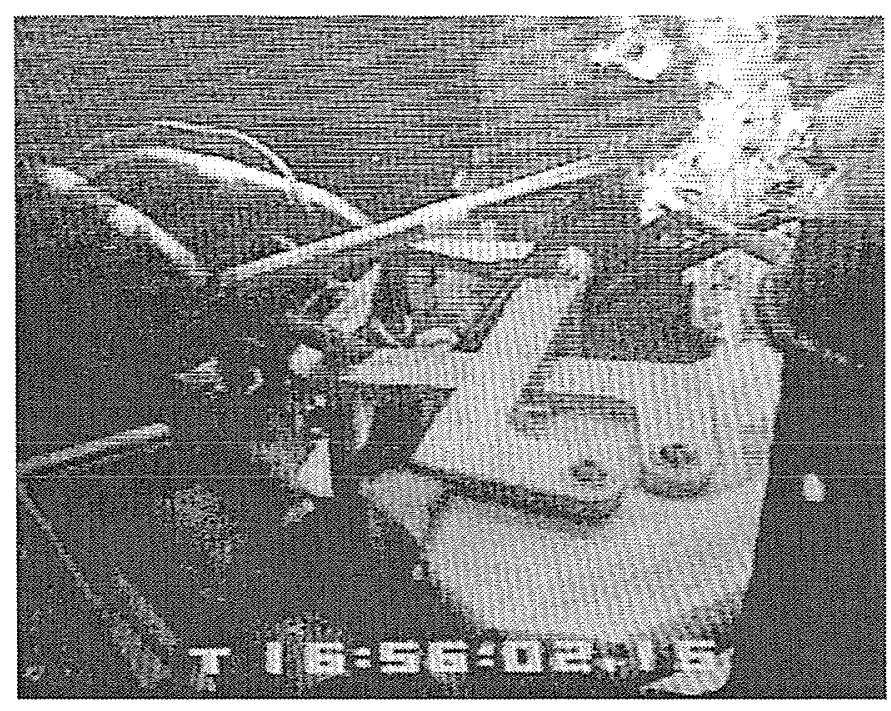

Figure 2-12: Gripper grasping water sampler. An X-handle attached to the sampler permits easy, secure grasping of the sampler. After placing the ends of the sampling tubes into the enlarged vent orifice the operator triggers the bottle to collect a sample of the $300+$ degree Centigrade plume water. (Outline added by authors). 


\section{Part II}

\section{Minimizing System Contributions to Misalignment}




\section{Chapter 3}

\section{Introduction}

To understand the motivation for the work presented in Part II, consider the problem shown in Figure 3-1 of using a manipulator arm attached to a free-flying underwater vehicle to accurately position an object or tool relative to a fixed underwater structure (for example, the vent water sampling task described in Chapter 2). One way to perform this task is to position the vehicle adjacent to the structure and try to position the object while hovering. Any forces applied by the manipulator to the environment result in equal and opposite reaction loads being applied to the vehicle. These reaction loads (in addition to loads due to water currents and/or the vehicle umbilical tether) tend to disturb the vehicle position, which in turn affects the position of the manipulator relative to the environment. This greatly complicates the completion of manipulation tasks.

To avoid this problem one could rigidly attach (i.e. dock) the vehicle to the structure. Docking, however, is only an option for tasks where we have the forethought and ability to install a docking fixture in the proper position and orientation ahead of time. A more flexible, intermediate option is to drive the vehicle up against the structure and exploit the contact forces between the vehicle and structure to, in effect, "contact dock" the vehicle. The contact forces constrain vehicle motion in certain directions but leave other vehicle motions unconstrained, yielding less misalignment 


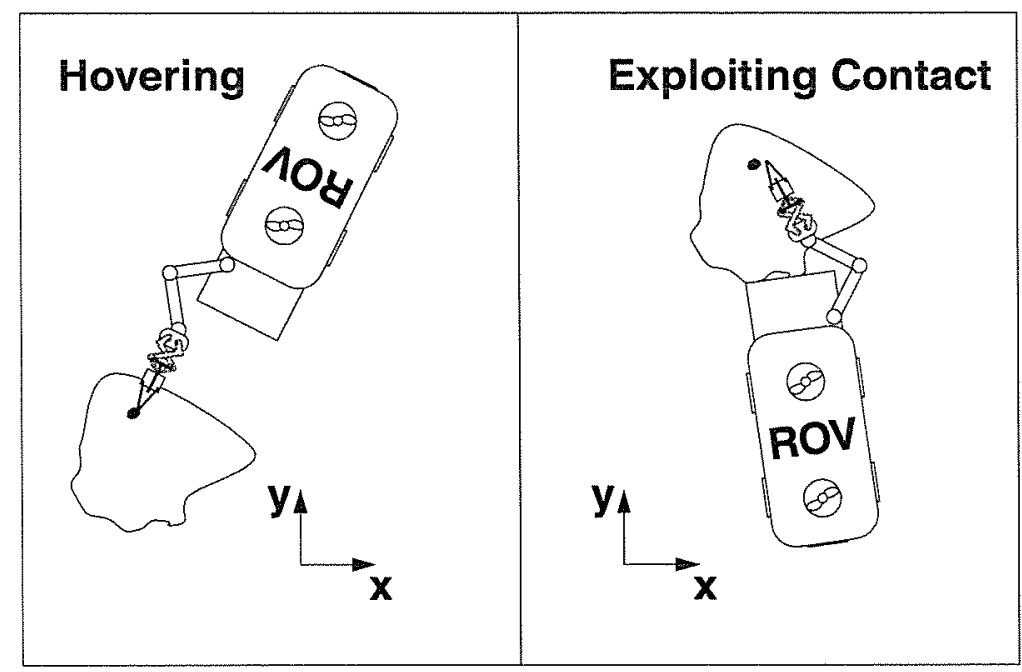

Figure 3-1: Remotely Operated Vehicle (ROV) based manipulator being used to perform a sampling task. In one case the vehicle hovers while the task is performed, in the other the vehicle exploits contact between the vehicle and a structure in the environment to stabilize the vehicle against manipulator reaction loads.

than hovering but much more flexibility than docking. The constraints imposed by contact, however, dramatically change the dynamics of the vehicle response to the thrust forces generated by its actuators. To maintain adequate control (or even stability) of the vehicle's remaining, unconstrained degrees of freedom the models used by the vehicle controller and, if applicable, by higher level task planning or supervisory control systems, must be updated to reflect these changes. Ideally the vehicle system should be able to perform this identification process automatically with no human intervention.

The remainder of this thesis considers the problem of automatically identifying the vehicle contact state (i.e. the locations of the points of contact (if any) and the resulting constrained and unconstrained degrees of freedom) from vehicle sensor data. In preparation, we briefly review existing, related work in the field and articulate how the contributions of this thesis complement this existing body of work. 


\subsection{Related Work}

\subsubsection{Control of Free-Flying Manipulation Systems}

Numerous controllers for underwater vehicles $[12,21,33,34]$ have been developed but almost all focus exclusively on the control of a fully unconstrained vehicle. A related application, the control of manipulators mounted to free-flying space vehicles has been studied extensively $[1,8,10,29,17]$, but this work also focuses almost exclusively on trajectory control of the manipulator endpoint when neither the manipulator nor the vehicle contact other objects in the environment.

This thesis considers a different problem, that of a vehicle partially constrained by contact with fixed objects in the environment. Specifically, we focus on determining the vehicle's contact state from the measurements of the vehicle's velocity and the net contact force experienced by the vehicle.

\subsubsection{Compliant Motion Control}

Fundamentally, a vehicle interacting with fixed environmental structures differs little from a manipulator interacting with fixed structures in its environment; both are examples of Compliant Motion Control, i.e. the control of a robot in contact with the environment [3]. Consequently, the contact identification scheme presented in this thesis, while described in terms of the vehicle problem, applies equally well to the general compliant motion control problem. The equivalency of the problems also permits us to draw upon the broad body of existing work in compliant motion control.

In a perfect world, compliant motion control strategies would be unnecessary. If we had perfect knowledge of the geometry and physical properties (e.g. stiffness, coefficient of friction, inertia properties, etc.) of the manipulator system and the environment and if we had perfect control of the manipulator position, we could perform any task by simply controlling the position of the manipulator. In the real world, however, our knowledge is far from perfect. For free (i.e. unconstrained) 
motions of the manipulator, small position errors, while undesirable, have little or no effect on the relationship between manipulator actuator forces and the resulting motion of the manipulator (we refer to this relationship as the manipulator model). When the manipulator is in contact (or nearly so) small position errors can lead to dramatic changes in the manipulator model because, due to the (typically) high stiffness of the manipulator and environment, very small position errors can result in very large contact forces. Thus very small errors in our knowledge of the manipulator or environmental geometry can lead to catastrophic results.

Compliant motion control strategies overcome this problem by minimizing the impact of errors in our knowledge of the system geometry. Two basic approaches have emerged. The Hybrid Control strategy introduced by Raibert and Craig [19] assumes that we know the directions in which contact constrains a manipulator's motion and partitions the overall control of the manipulator into two mutually orthogonal control problems; the control of forces in the constrained directions and the control of position in the unconstrained directions. As long as the assumed constraints closely match the actual constraints, errors in the position control sub-problem have minimal impact on the force control sub-problem, and vice versa. This work is based upon the concepts of natural and artificial constraints presented by Mason [18].

The Impedance Control Strategy presented by Hogan [14] imposes, through design of the controller, a set of desired physical properties ( i.e. generalized inertia, damping and stiffness) on a manipulator which specify the end effector's deviation from a reference position or trajectory in response to disturbance loads. Specifying low impedance in constrained directions and high impedance in unconstrained directions minimizes the impact of geometric uncertainty while permitting accurate position control in the unconstrained directions. An early example of an impedance type controller was the active stiffness control presented by Salisbury [22].

Note that both approaches assume that we know the directions in which the environment constrains the manipulator. As such, these strategies cannot be used in 
unstructured environments (i.e. environments where we have little or no knowledge of the geometry of objects and environment) unless we have some means by which we can determine this information. The contact identification technique presented in this thesis enables the use of these established compliant motion control techniques in unstructured environments by determining a moving object's constrained directions (and, in general, the locations of it's points of contact with the environment) from measurements of the object's position, velocity and the net force experienced by the object as a result of contact with the environment. Figure 3-2 shows how such a contact identification system could be integrated with a hybrid controller and a

higher level controller. Note that the role of the identification system is that of a sensor: it does not directly update the hybrid controller constraint information, it simply makes information available to the higher level controller. The higher level controller decides, based on the task, what action (if any) to take in response to this information. This thesis focuses exclusively on the problem of identification.

\subsubsection{Bracing}

The motivating example for this thesis, that of a vehicle exploiting contact with the environment to help stabilize the vehicle position against the effects of disturbance loads, is an example of bracing. West [32] presented a comprehensive analysis of the effects of bracing on a manipulator's kinematic, static and mechanical properties. This work, geared towards improving manipulator performance in machining and grinding tasks, assumes the constraints imposed by bracing are known and considers the problem of determining the ensuing properties and of designing braced manipulators which yield desired performance characteristics. For the vehicle bracing problem considered in this thesis, West's work becomes applicable only after we have identified the contact state. The contact identification system presented in this thesis, when coupled with a hybrid controller as shown in Figure 3-2, will enable the exploitation of bracing in unstructured environments. 


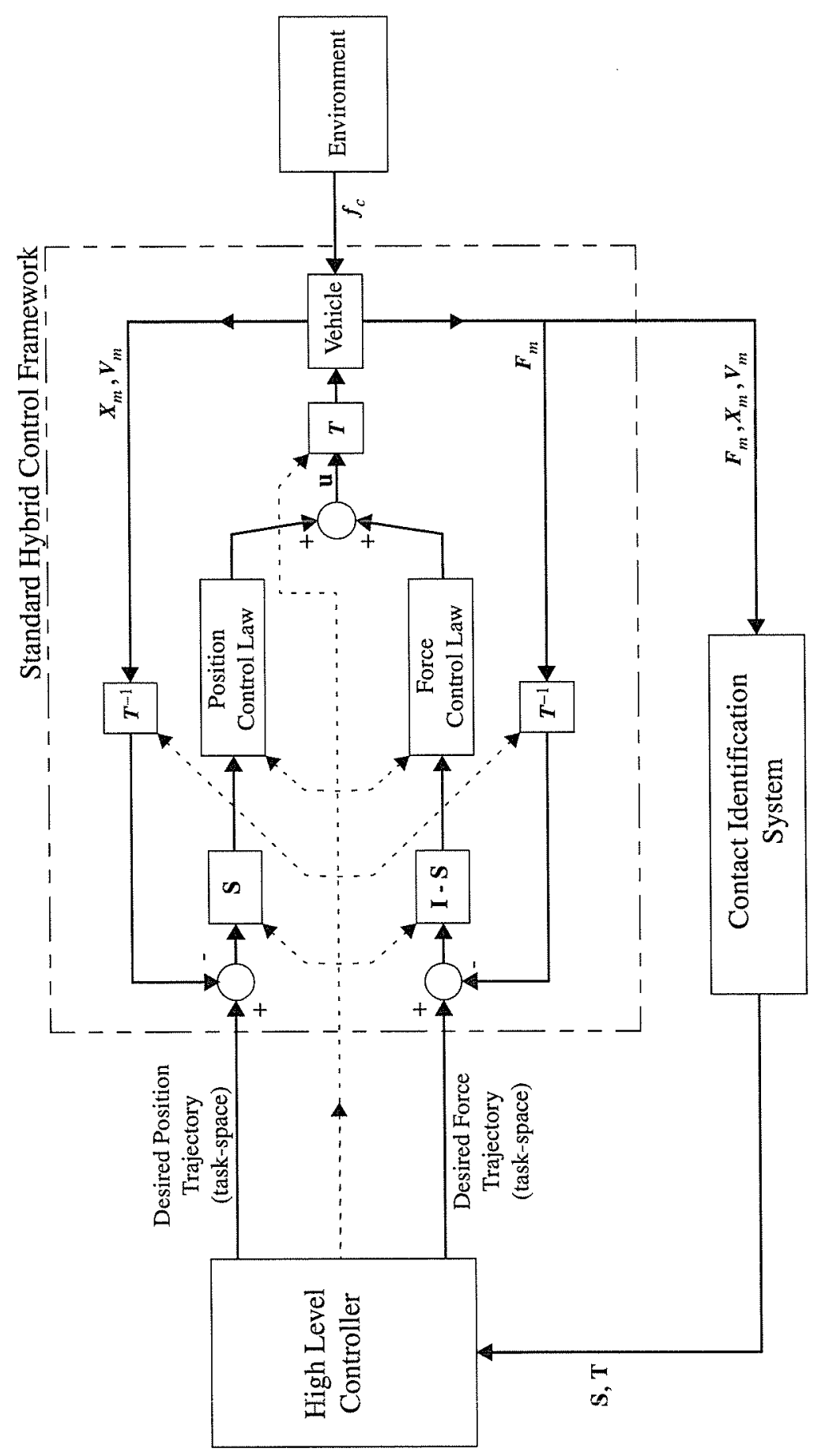

Figure 3-2: Incorporation of a contact identification system with the hybrid control framework. Note that the identification system plays the role of a sensor, i.e. it simply makes information available, it does not directly update any system parameters. 


\subsubsection{Intrinsic Contact Sensing}

Intrinsic contact sensing uses measurements of the net force and torque experienced by an object in conjunction with knowledge of the object's geometry to determine, in the case of a single contact force, the location of the contact and local surface properties (e.g. surface normal, coefficient of friction, etc.). This concept is introduced by Salisbury [23] in the context of the design of a fingertip contact force sensor. Bicci et.

al. [5] define and solve for the location of the contact centroid for the case of a single soft finger in contact with a convex body. These approaches are inherently limited to the case of contact at a single point ( or, in the case of the soft finger, at a signle contact region). We will find that locating contact points for the case of multiple contacts requires that we also consider the velocity of the actuated object.

\subsubsection{Fixturing}

The vehicle bracing problem is similar in some respects to that encountered in work on fixturing, i.e. we are considering the constraints imposed on a planar rigid body as a result of contact with other fixed, planar rigid bodies. Asada and By [2] presented a method to determine if a particular fixture design fully constrains a rigid body. Brost and Goldberg [6] present an algorithm which, given a two-dimensional part geometry, selects the optimal fixture design from the set of all fixture designs which are possible using a modular fixturing system. Schimmels and Peshkin [25] present a method for designing a manipulator admittance matrix (to be realized by the manipulator controller) which leads to contact forces that always act so as to reduce the degree of misalignment between a part and the fixture into which it is being guided.

The basic formulation of an object's kinematic and static constraint equations is identical for the contact identification and fixturing problems, but the underlying assumptions are different. In fixturing problems, contacts are generally assumed to be frictionless, as any fixture which imposes full constraint for the frictionless case is guaranteed to impose full constraint in the case of non-zero friction as well. In 
the contact identification problem, however, friction cannot be ignored as it is responsible for a significant component of the measured net interaction force. Even more fundamentally, fixturing work largely considers the characteristics of a stationary object while contact identification, as we will see, is primarily concerned with the relationship between an object's measured interaction force and its measured velocity.

\subsubsection{Contact Identification}

Several researchers have examined the problem of identifying the constraints imposed on a rigid body by contact with a fixed environment. Bruyninckx [7] models contact by constructing a virtual manipulator for each of a body's points of contact. Each virtual manipulator imposes constraints equivalent to those imposed by the contact itself. Bruyninckx presents an identification scheme which continuously modifies the parameters of the virtual manipulators so that the contact points they represent satisfy the reciprocity constraint on the power dissipated at the contact point. The technique yields both first order (i.e. contact point location, contact normal) and second order (i.e. curvature) properties at the contact points. The technique assumes, however, that the number and type of contacts are known and thus is not a fully general contact identification system. The technique presented in this thesis differs most significantly from Bruyninckx's technique in that makes no assumptions about the number or types of contact points and therefore qualifies as a general contact identification algorithm.

Perhaps the most comprehensive contact identification work performed to date is that of Eberman [9]. Eberman describes a general identification algorithm which makes statistically optimal use of the measured force and velocity data in identifying the current contact model. This optimality, however, comes at the cost of a significant computational burden, i.e. the need to iteratively solve a set of coupled eigenvalue-like problems for each possible contact model in every identification cycle. The technique was experimentally verified with excellent results for a point object moving in a planar 
environment. An extended version covering the motion of polygons was tested using simulated data to determine the accuracy with which a body's constrained directions could be determined when the correct model was known. No testing of the polygonal version's ability identify the correct model from the set of all possible candidate models was performed.

The technique presented in this thesis differs from Eberman's technique in that, by settling for a non-optimal solution it reduces the necessary computation per candidate model to the solution of two least squares problems. In addition, this thesis presents experimental results confirming the technique's ability to determine the true contact state from the set of all possible candidate contact models.

\subsection{Problem Statement}

This thesis addresses the problem of determining the contact state of a moving, planar rigid body interacting with fixed, planar rigid bodies in its environment. By contact state we mean the locations of the points of contact and the directions in which the contact constrains the vehicle motion. Table 3.1 summarizes the assumptions upon which this work is based.

\subsection{Contributions of Part II}

- A new, computationally efficient technique which identifies the contact state of a moving planar rigid body interacting with fixed but otherwise unknown planar rigid bodies in its environment. The technique takes as inputs the moving body's measured position, velocity and net interaction force and assumes that the body's geometry and the coefficient of dynamic friction acting between the body and the environment are known. The outputs are the force and velocity constraints acting on the vehicle and, in most cases, the locations of the vehicle's 
actual points of contact.

- The concept and formulation of the violation power and violation energy as suitable metrics for choosing between possible contact models.

- Experimental verification of the proposed contact identification technique.

\subsection{Overview}

\subsubsection{Synopsis of Approach}

Initially, we have no knowledge of the vehicle's true contact state. Every point on the vehicle perimeter is a possible contact point, giving rise to an infinite number of possible contact scenarios and their corresponding contact models.

The velocity constraint imposed by a point of contact between the vehicle and a fixed object in the environment specifies that the velocity of the vehicle at this point must be zero in the direction of the vehicle's outward facing normal at that point. Thus, given a measurement of the vehicle velocity and knowledge of the vehicle boundary geometry, we can solve for the set of points whose velocities satisfy this condition. We refer to the set of all such points as the vehicle's kinematically feasible candidate contact points or, for brevity, as the candidate contact points. These are the vehicle's only possible points of contact with fixed objects in the environment, i.e. the actual contact points must be members of this set.

By considering all possible unique combinations of candidate contact points we can construct the set of all possible kinematically feasible candidate contact models, of which the actual contact model is necessarily a member. Each candidate contact model consists of a set of presumed contact points and the two sets of constraint equations that result from imposing the rigid body contact constraints (discussed in Chapter 4) to these points. The model's force constraint equations relate the forces acting at the presumed contact points to the net force and moment measured by a vehicle 
mounted force sensor. The model's velocity constraint equations relate the velocities at the presumed contact points to the velocity measured by a vehicle mounted velocity sensor.

The vehicle's measured data result from the physical interactions taking place at the actual contact points; therefore we expect the measured force and velocity to be consistent with (i.e. to satisfy) the force and velocity constraints associated with the contact model describing the actual contact state. Since this model is necessarily a member of the set of candidate contact models, we can use the degree to which the measured data violates each model's constraint equations to identify the correct model.

There are two aspects to the violation of a model's constraint equations; feasibility and consistency. Using the the measured force and moment we can solve for the corresponding quasistatic reaction loads at each model's presumed contact points. A model is quasistatically feasible if these reaction loads satisfy the rigid body constraint on each contact force (i.e. that objects can only "push" on each other, they cannot "pull"). Thus we discard from consideration all infeasible candidate contact models.

We test the remaining feasible candidate contact models for consistency. A candidate model's constraint equations define a permissable force space and a permissable motion space for the model containing, respectively, the measured forces and velocity vectors which the candidate model is physically capable of producing. By projecting the measured data into each of these vector spaces we can, for each candidate contact model, decompose each measured data vector into the sum of a permissable vector and an impermissable vector. The elements of the impermissible vectors indicate the degree to which the measured data violate each of a model's force and velocity constraint equations. From these components we compute the violation power, i.e. the power dissipation associated with violation of each of a model's constraint equations. By properly combining the individual violation power terms for each constraint equation we form a positive definite consistency measure by which we can assess the 
degree of compatibility between each candidate model and the current measured data vectors. The violation power based metric, however, only identifies the best model within a model class. To identify which of the best-of-class models is the best overall model, we introduce a similar metric based on the violation energy, i.e the work associated with the violation of a models constraint equations during a given incremental motion of the vehicle. Finally, we demonstrate experimentally the efficacy of this two stage identification process.

\subsubsection{Guide to Part II}

\section{Chapter 4: Identifying Kinematically Feasible Candidate Contact Points}

Reviews the characteristics of rigid body contact. Develops and experimentally verifies a technique to identify all candidate contact points, i.e. points on the vehicle boundary which, based on kinematic considerations alone, could be in contact with a fixed object in the environment.

\section{Chapter 5: Constructing Kinematically Feasible Candidate Contact Models}

Considers the complete set of candidate contact models which can be constructed from the set of candidate contact points. Describes the five classes into which these models fall and articulates the force and velocity constraints associated with each model class.

\section{Chapter 6: Identifying the Best Candidate Contact Model Within Each Class}

Defines the concepts of model feasibility and model consistency. Introduces the violation power and demonstrates its essential role in determining model feasibility, model consistency and in identifying the best candidate model within each model class. Articulates why a violation power based approach by itself cannot identify the best overall model.

Chapter 7: Identifying the Best Overall Model Introduces the violation energy and demonstrates how, in conjuntion with the results of the violation power 
based approach, it permits the identification of the best overall model.

Chapter 8: Experimental Verification Presents experimental results verifying the ability of the violation power based approach to identify the best model in each class. Presents experimental results verifying the ability of the violation energy based approach to identify the best overall model. Investigates the sensitivity of the technique to errors in the assumed value of the coefficient of dynamic friction.

Chapter 9: Conclusions Summarizes the key results of the thesis. Discusses future work.

\subsubsection{Assumptions}

The assumptions in Table 3.1 apply throughout the remainder of this thesis. 


\section{Assumptions}

The vehicle...

- is a planar, rigid body.

- has a known, piece-wise continuous, closed bounding curve.

- has a known coefficient of dynamic friction acting at points of contact between the vehicle and the environment which is the same at all points of contact.

Objects in the environment ...

- are planar, rigid bodies.

- have unknown piece-wise continuous boundary curves.

- have unknown position and orientation in relation to a fixed world reference frame.

- are rigidly fixed in relation to the fixed world reference (i.e. their linear and angular velocities are known and equal to zero ).

We can measure ...

- the vehicle's position and orientation in relation to a fixed world reference frame.

- the vehicle's linear and angular velocity in relation to the fixed world reference frame.

- the aggregate force and moment applied to the vehicle as a result of the (unknown) forces acting at the (unknown) points of contact with the environment.

Table 3.1: Assumptions for Part II 


\section{Chapter 4}

\section{Identifying Kinematically Feasible Candidate Contact Points}

\subsection{Introduction}

In this chapter we develop and test a technique which, given the geometry and velocity of a planar, rigid body (the vehicle) interacting with fixed obstacles (the environment), identifies the set of possible contact points on the body. Section 4.2 begins with a review of the characteristics of points of contact between rigid bodies. In Section 4.3 we exploit this information to define and mathematically solve for kinematically feasible candidate contact point locations on two types of vehicle boundary curves; straight lines and circular arcs. Section 4.4 experimentally investigates the error between the computed candidate contact point locations and the actual contact points for various contact configurations for a body composed of straight line and circular arc boundary curves. 


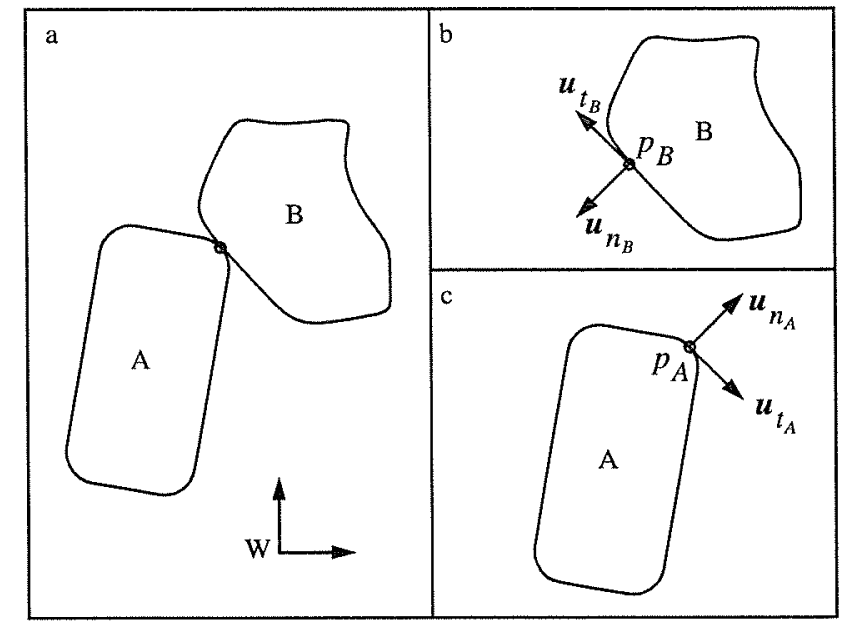

Figure 4-1: (a) Two planar rigid bodies in contact. (b) Tangent vector and outward facing normal at contact point $p_{B}$ on body B. (c) Tangent vector and outward facing normal at contact point $p_{A}$ on body $\mathrm{A}$.

\subsection{Properties of Planar Rigid Body Contact}

Figure 4-1 shows two arbitrary planar rigid bodies $A$ and $B$ in contact at a single point. For the time being, consider both bodies to be free to move, i.e neither body is fixed. A point $p_{A}$ on the boundary of body $\mathrm{A}$ and a point $p_{B}$ on the boundary of body $\mathrm{B}$ are in contact when

1. point $p_{A}$ coincides with point $p_{B}$, i.e. $p_{A}=p_{B}$

2. the tangent vectors at points $p_{A}$ and $p_{B}$ are parallel and coincident.

3. the outward facing normal vectors at points $p_{A}$ and $p_{B}$ are coincident but opposite in direction

4. the normal component of the relative velocity of points $p_{A}$ and $p_{B}$ is equal to zero, i.e. $\left(\mathbf{v}_{p_{A}}-\mathbf{v}_{p_{B}}\right) \cdot \mathbf{n}_{p_{A}}=\left(\mathbf{v}_{p_{A}}-\mathbf{v}_{p_{B}}\right) \cdot \mathbf{n}_{p_{B}}=0$

5. the force applied to each object through the contact point has a negative component in the direction of that object's outward facing normal at the point of 
contact, i.e. $\mathbf{f}_{p_{A_{\text {contact }}}} \cdot \mathbf{n}_{p_{A}}<0$ and $\mathbf{f}_{p_{B_{\text {contact }}}} \cdot \mathbf{n}_{p_{B}}<0$ (in other words, objects can only "push" on each other through a contact, they cannot pull ).

Note that, given characteristic five, two objects with coincident boundary points are not considered to be in contact unless the force transmitted between them is nonzero.

\subsection{Zero-Normal-Velocity (znv) Points}

Given our assumption that all objects in the environment are fixed, we may treat the entire environment as a single rigid body composed of the union of all its component objects. Being fixed in space, the velocity of every point on this aggregate environmental object is equal to zero. If we let the vehicle be object $A$ and let the environment be object $B$ then characteristic four in the preceding list becomes

$$
\mathbf{v}_{p_{A}} \cdot \mathbf{n}_{p_{A}}=0
$$

The modified characteristic states that the only points on the vehicle boundary which could possibly be points of contact with a fixed environment are points which have zero velocity in the direction of their local boundary normal. If we know 1) the geometry of the vehicle boundary and 2) the linear and angular velocity of a point on the vehicle we can solve for this set of points analytically. We refer to these points interchangeably as zero-normal-velocity (znv) points or as candidate contact points. To solve for the locations of these points we shall exploit the concept of the instantaneous center of rotation. 


\subsubsection{The Instantaneous Center of Rotation}

Imagine for the moment that our vehicle has infinite expanse, i.e. it is an infinite plane which moves with respect to the fixed world plane. The instantaneous center of rotation (ICR) is a unique point (in the moving plane) which has zero linear velocity with respect to the fixed plane at the given instant in time. The motion of every other point on the moving plane can be represented as resulting from a pure rotation of the vehicle about the ICR. Given the velocity of a point $p_{i}$ in the vehicle plane, the velocity of another point $p_{j}$ in that plane is

$$
{ }^{W} \mathbf{v}_{p_{j}}={ }^{W} \mathbf{v}_{p_{i}}+\left[{ }^{W} \mathbf{r}_{i j} \times\right] \omega
$$

where ${ }^{W} \mathbf{v}_{p_{j}}$ and ${ }^{W} \mathbf{v}_{p_{i}}$ are the velocities of points $i$ and $j$ with respect to the fixed world frame $W,{ }^{W} \mathbf{r}_{i j}$ is the vector from $p_{i}$ to $p_{j}, \omega$ is the angular velocity of the vehicle plane and

$$
\left[\mathbf{r}_{i} \times\right]=\left[\begin{array}{c}
-r_{i_{y}} \\
r_{i_{x}}
\end{array}\right]
$$

is a convenient notation for expressing the planar form of the cross product (see the Mathematical Notation appendix at the end of this thesis for a more detailed discussion of this notation).

We can rewrite equation 4.2 with respect to an alternate world-fixed frame $V W$, defined to be a frame which conicides, instantaneously, with a corresponding vehiclefixed frame $V$. In this case we get

$$
{ }^{V W} \mathbf{v}_{p_{j}}={ }^{V W} \mathbf{v}_{p_{i}}+\left[{ }^{V W} \mathbf{r}_{i j} \times\right] \omega
$$

where each of the terms is now written with respect to fixed frame $V W$. 
To locate the ICR with respect to frame $V$ we set ${ }^{V} \mathbf{v}_{p_{j}}={ }^{V} \mathbf{v}_{p_{I C R}}=0$ and solve for ${ }^{V} \mathbf{r}_{i j}={ }^{V} \mathbf{r}_{i I C R}$ (i.e. the vector from $p_{i}$ to the ICR). If we also let $p_{i}$ be the origin of the vehicle fixed frame, the location of the ICR with respect to the vehicle fixed frame is

$$
{ }^{v} \mathbf{r}_{I C R}=\left(\left[\begin{array}{c}
-v_{y_{O}} \\
v_{x_{O}}
\end{array}\right] \frac{1}{\left|{ }^{V} \mathbf{v}_{p_{O}}\right|}\right)\left(\frac{\left|\mathbf{v}_{p_{O}}\right|}{\omega}\right)
$$

The first term in parentheses is a unit vector, orthogonal to the velocity vector at point $i$, which gives the direction of ${ }^{V} \mathbf{r}_{i j}$. The second term is the positive or negative distance between point $i$ and the ICR in the direction of the unit vector.

There are three special case solutions for ${ }^{V} \mathbf{r}_{i j}$. When the magnitude of the linear velocity $\left|V^{V} \mathbf{v}_{p_{i}}\right|=0$, the ICR is simply $p_{i}$. When $\left|{ }^{V} \mathbf{v}_{p_{i}}\right| \neq 0$ but $\omega=0$, the unit vector is still defined but the magnitude term becomes infinite. If both $\left|{ }^{V} \mathbf{v}_{p_{i}}\right|$ and $\omega=0$, the vehicle is stationary and the ICR does not exist. Note that if we let $p_{i}$ be the origin of the vehicle frame

Now consider that, instead of being an infinite plane, our vehicle has a finite boundary. The ICR still exists, but it will not necessarily lie within the domain of the vehicle boundary.

As stated above, the only points on the boundary that could be in contact with fixed objects in the environment are those which have zero velocity in the direction of their outward facing normal. Given that the velocity of any point on the vehicle is equivalant to pure rotation about the ICR, this condition will be satisfied for any boundary point whose normal vector, when extended, passes through the ICR. Mathematically, znv points are points on the vehicle boundary which satisfy

Now consider that, instead of being an infinite plane, our vehicle has a finite boundary. The ICR still exists, but it will not necessarily lie within the domain of the vehicle boundary. As stated above, the only points on the boundary that could be in contact with fixed objects in the environment are those which have zero velocity 
in the direction of their outward facing normal. Given that the velocity of any point on the vehicle is equivalant to pure rotation about the ICR, this condition will be satisfied for any boundary point whose normal vector, when extended, passes through the ICR. Mathematically, znv points are points on the vehicle boundary which satisfy

$$
\mathbf{r}_{b}=\mathbf{r}_{I C R}+s_{n} \mathbf{u}_{n}\left(\mathbf{r}_{b}\right)
$$

where $\mathbf{r}_{b}$ is the position vector of a point on the boundary, $\mathbf{u}_{n}\left(\mathbf{r}_{b}\right)$ is the outward facing normal vector at that point, and $s_{n}$ is the distance between the ICR and point $\mathbf{r}_{b}$.

This is illustrated graphically in Figure 4-2.a for a rectangular vehicle with rounded corners. Figures 4-2.b through 4-2.d show this vehicle's znv points for several different locations of the ICR. To find a given boundary segment's znv point we draw a radial line from the ICR to the segment such that the radial line is orthogonal to the boundary segment at the point of intersection.

\subsubsection{Locating znv points on straight line boundary segments}

Let us represent points on the line segment as

$$
\mathbf{r}_{\mathbf{b}}=\mathbf{r}_{o}+s_{t} \mathbf{u}_{t}, \quad 0 \leq s \leq s_{\max }
$$

where $\mathbf{r}_{o}$ is the segment's start point, $\mathbf{u}_{t}$ is a unit vector pointing from the start point towards the end point, $s_{t}$ is the distance between point $\mathbf{r}_{b}$ on the line and the start point. The normal to the line is everywhere equal to

$$
\mathbf{u}_{n}=\left[\begin{array}{c}
-u_{t_{y}} \\
u_{t_{x}}
\end{array}\right]
$$



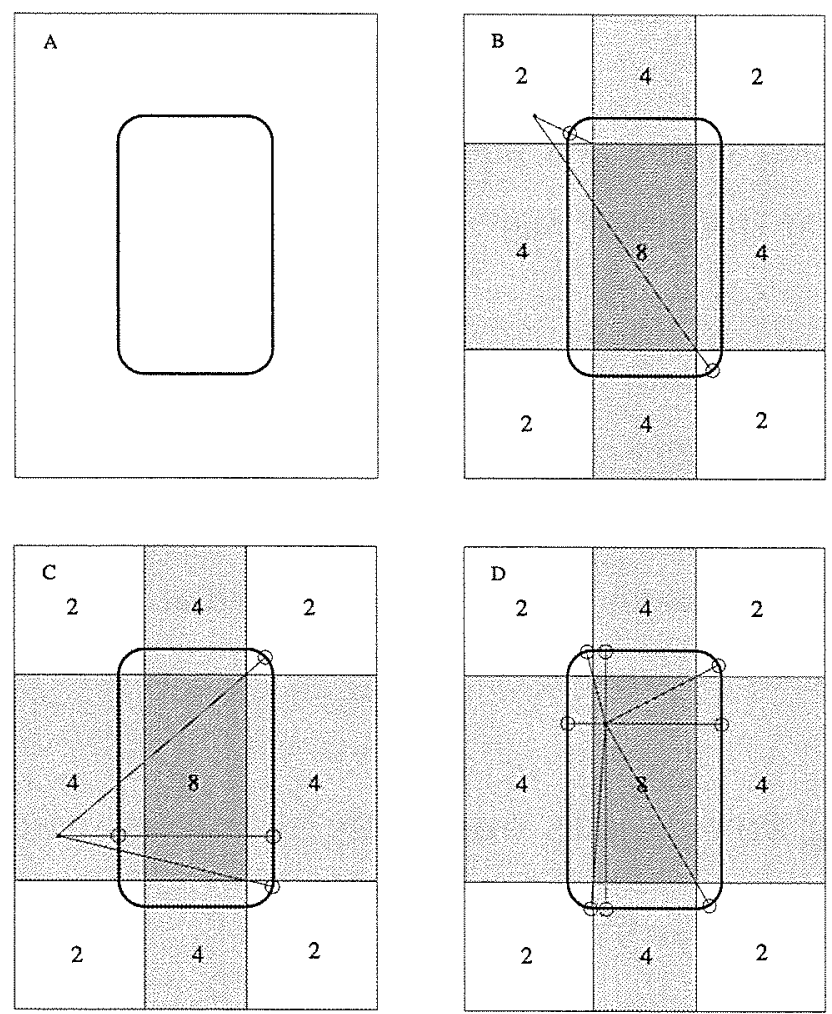

Figure 4-2: The number in each region indicates the number of points on the vehicle perimeter (heavy black line) for which $v_{n}=0$ when the instantaneous center of rotation (ICR) lies within that region. For example, when the ICR is at the indicated position there are eight points which have zero velocity in the direction normal to the local surface tangent. For the given vehicle state and geometry, these are the only possible points of contact with stationary objects in the environment. 
Using equations 4.7 and 4.8 to eliminate $\mathbf{r}_{b}$ and $\mathbf{u}_{n}\left(\mathbf{r}_{b}\right)$, equation 4.6 becomes

$$
\mathbf{r}_{o}+s_{t} \mathbf{u}_{t}=\mathbf{r}_{I C R}+s_{n} \mathbf{u}_{n}
$$

Solving for $s_{t}$ and $s_{n}$ yields

$$
\begin{aligned}
{\left[\begin{array}{c}
s_{t} \\
s_{n}
\end{array}\right] } & =\left[\left[\mathbf{u}_{n}\right],\left[-\mathbf{u}_{t}\right]\right]^{-1}\left(\mathbf{r}_{o}-\mathbf{r}_{I C R}\right) \\
& =\left[\left[\mathbf{u}_{n}\right],\left[-\mathbf{u}_{t}\right]\right]^{T}\left(\mathbf{r}_{o}-\mathbf{r}_{I C R}\right)
\end{aligned}
$$

Substituting $s_{t}$ into Equation 4.7 yields the location of the znv point for the line. This znv point is a valid candidate contact point only if $0 \leq s_{t} \leq s_{\max }$, i.e. if it lies within the domain of the boundary segment. Thus, in general, there is a unique znv solution for each line segment which contributes at most one unique candidate contact point per line segment.

The only exception occurs when the angular velocity is equal to 0 AND the linear velocity vector is parallel to the direction of the line. In this case, the distance between the line and the ICR in the line's normal direction is infinite and as a result every point on the line has zero velocity in the normal direction. We will discuss the implications of this special case as it relates to the selection of the best contact model in chapter 8 .

\subsubsection{Locating znv points on circular arc boundary segments}

Figure 4-3 shows a portion of a vehicle boundary which includes an are segment. The arc segment's underlying circle has radius $R_{a}$ and centerpoint $\mathrm{C}$. The arc segment is the portion of the circle obtained by traversing from point $s$ to point $e$ in a clockwise fashion. All arcs segments in this thesis are defined in a clockwise sense.

To find the arc segment's znv points we first find the znv points for the underlying 


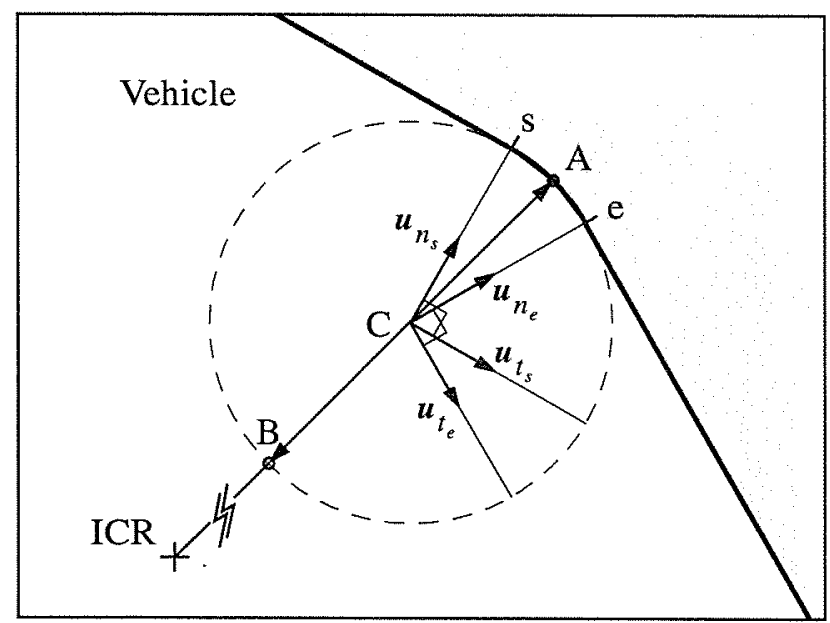

Figure 4-3: There are generally two znv points ( points A and B ) associated with a circular arc boundary segment, these being the intersections of the line drawn from the ICR to the arc center $\mathrm{C}$ with the arc's underlying circle. Only solutions which lie within the domain of the arc qualify as candidate contact points. An intersection point lies in the domain of the arc if the vector $\mathbf{r}_{p}$ from the arc center $\mathrm{C}$ to the point satisfies the conditions in Equation 4.14

circle and then determine which, if any, of these lie within the domain of the arc segment. The underlying circle's znv points are simply the intersection points between an extended line drawn from the ICR through the center point $\mathrm{C}$ and the circle itself ( here, points $\mathrm{A}$ and $\mathrm{B}$ ). These are the only points on the circle whose local normal vectors, when extended, pass through the ICR. Defining $\mathbf{u}_{n}$ to be a unit vector in the direction of this line the znv point locations are

$$
\mathbf{r}_{z n v}=\mathbf{r}_{C} \pm R_{a} \mathbf{u}_{n}
$$

A given point lies in the domain of an arc segment if its angular position, relative to the arc center, falls between the start and end angles. This test is complicated by the discontinuity that occurs in the angle returned by the arc-tangent function, i.e. arc-tangent functions typically return values that range from $-\pi$ to $+\pi$. Assuming an 
arctangent function of this form, arcs defined in a clockwise fashion have start angles which are larger than the end angles except when the arc straddles the discontinuity, in which case the start angle is less than the end angle.

It is more convenient to use a vector based domain test. This approach avoids the discontinuity problem. A point on the circle lies in the domain of the arc if, given the vector $\mathbf{r}_{C p}$ from the arc center $\mathrm{C}$ to the point, the following conditions are all true:

$$
\begin{aligned}
\mathbf{r}_{C p} \cdot \mathbf{u}_{t_{s}} & \geq 0 \\
\mathbf{r}_{C p} \cdot \mathbf{u}_{n_{s}} & \geq 0 \\
\mathbf{r}_{C p} \cdot \mathbf{u}_{t_{e}} & \leq 0 \\
\mathbf{r}_{C p} \cdot \mathbf{u}_{n_{e}} & \geq 0
\end{aligned}
$$

where $\mathbf{u}_{t_{s}}, \mathbf{u}_{n_{s}}$ and $\mathbf{u}_{t_{e}}, \mathbf{u}_{n_{e}}$ are the tangential and normal unit vectors evaluated at points s and e respectively.

In general there are two znv point solutions for the underlying circle. If we restrict arc segments to angular domains of less than $\pi$ radians, no more than one of these solutions will lie in the segment's domain. For many ICR locations, neither znv point will lie in the domain of the arc. This is the case, for example, for the lower left and upper right arcs in Figure 4-2 B. Thus, in general, there will be two znv points but, at most, one unique candidate contact point per arc segment.

The only exception occurs when the ICR coincides with the arc center C. In this case the number of candidate contact points is infinite as every point on the arc segment has zero velocity in the normal direction. Again, we will discuss the implications of this special case as it relates to the selection of the best contact model in chapter 8 . 


\subsubsection{Locating znv points on composite curves}

In principle we can determine the znv locations for a wide variety of geometric elements. Without loss of generality, however, we restrict ourselves in this thesis to considering bodies which are comprised of line and arc segments. For example, the vehicle boundary shown in Figure 4-2 A combines four line segments and four arc segments to form a smooth, continuous closed composite curve. The set of znv points for this vehicle is simply the union of the znv points of its component segments.

\subsection{Experimental Results}

Several experiments were run to investigate the accuracy with which the znv point locations could be determined. Figure 4-4 shows the Air Table Vehicle Simulator (ATVS) system, the experimental apparatus constructed for these and the other experiments performed in this thesis ( see Appendix A for a detailed description of the system design and its characteristics ). Briefly, the system consists of a vehicle which, supported by three air bearings, moves freely over the surface of a one meter square glass topped table. Four miniature steel cables couple the motion of the vehicle to that of four brushless D.C. servomotors mounted to the table corners. Optical encoders measure the rotation angle and velocity of each motor, enabling the determination of the vehicle position/orientation and velocity relative to the table. For the experiments in this chapter the vehicle was moved by hand, i.e. the motors were only used to maintain nominal tensions in the cables. The vehicle boundary is identical to that shown in Figure 4-2 A.

For these tests, no filtering was performed on the vehicle velocity data, i.e. the ICR location and the znv point locations were computed directly from the raw vehicle world frame velocity vector. The vehicle world frame velocity vector was computed from the vector of motor shaft velocities as described in Appendix A. 


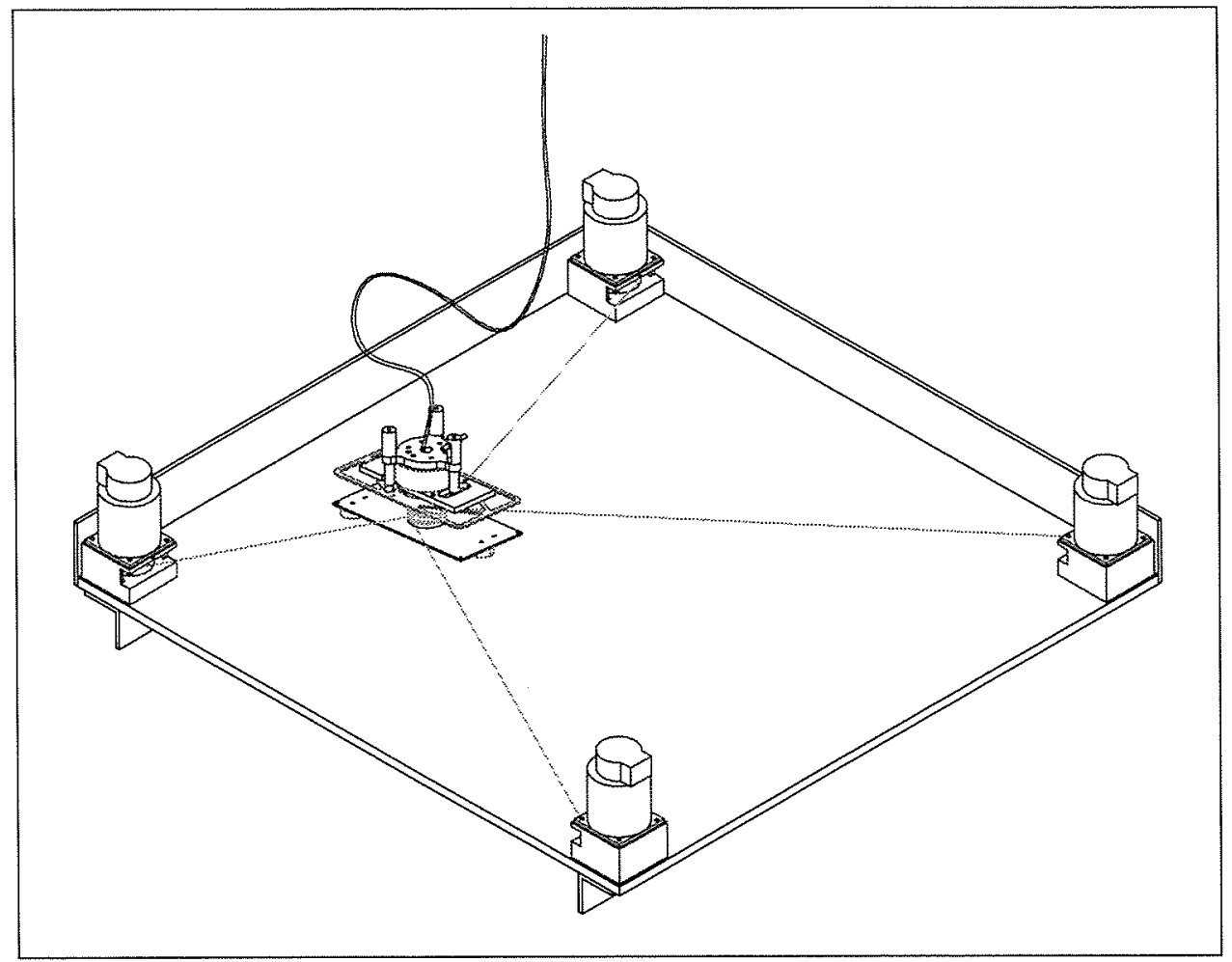

Figure 4-4: The Air Table Vehicle Simulator (ATVS) system consists of a 0.145 by 0.29 meter, air bearing supported vehicle which moves freely over the surface of a one square meter glass topped table surface. Four miniature steel cables couple the motion of the vehicle to that of the four motors mounted to the corners of the table. In general, the position and orientation of the vehicle is controlled by coordinating the motion of the four motors. The position and orientation, as well as the linear and angular velocities, of the vehicle are determined from the motor shaft positions and angular velocities as measured by optical encoders mounted to the motor shafts. For all the experiments performed in this chapter, however, the vehicle was moved by hand, i.e. the actuators were only used to maintain tension in the cables. This system is described in greater detail in Appendix A 


\subsubsection{Case 1: One Point Contact with No Slip (Pure Rota- tion)}

As shown in Figure 4-6 edge 5 of the the vehicle was brought into contact with corner c1 of a fixed obstacle in the environment. The vehicle was rotated about this corner in a counter clockwise direction from time $t_{1}$ to time $t_{2}$, at which time the rotation of the vehicle was stopped and then reversed. At all times the rotation of the vehicle was performed such that no slip occurred at the corner. Figure 4-6 is a plot of the distance between the computed location of the edge $5 \mathrm{znv}$ point and the known location of the corner. During normal motion the computed location of the znv point is seen to stay within $5 \mathrm{~mm}$. of the actual contact point. The error only extends beyond this range near the velocity reversal, i.e. when the angular velocity is near zero. Since no slip occurs at the contact point, zero angular velocity means that the vehicle is at a complete standstill. In such a case the location of the ICR does not exist so the znv points do not exist either, so we expect the accuracy with which the znv point tracks the actual contact point to degrade. Once the vehicle begins moving in the opposite direction, the tracking accuracy returns to the $5 \mathrm{~mm}$. accuracy range.

\subsubsection{Case 2: One Point Contact with Slip (Rotation and Translation)}

In Case 2 edge 5 is again brought into contact with corner c1 (see Figure 4-7) but this time the vehicle is moved such that sliding occurred at the contact point. The overall motion involved combined translation and rotation of the vehicle. Motion of the vehicle was initiated at time $t=0$ and continued until time $t_{2}$ at which point contact with the corner was broken. Figure 4-8 plots the distance between the computed location of the znv point for edge 5 and the known location of corner ci, indicating that the tracking accuracy is roughly $10 \mathrm{~mm}$ during the motion of the vehicle. 


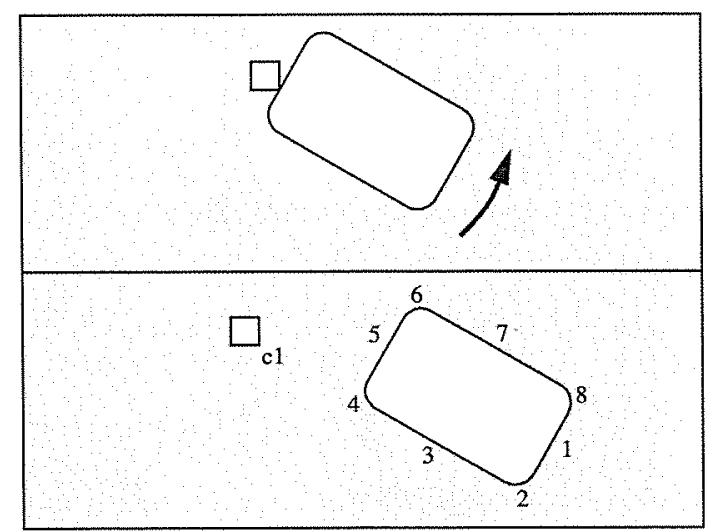

Figure 4-5: Case 1 Experiment: Edge 5 of the vehicle was brought into contact with corner $\mathrm{cl}$ of a fixed object in the environment. The vehicle was then rotated about this corner such that little or no slip occurred. Initial rotation was in the counter clockwise direction, then the rotation was briefly stopped and then reversed, again maintaining minimal slip at the contact point

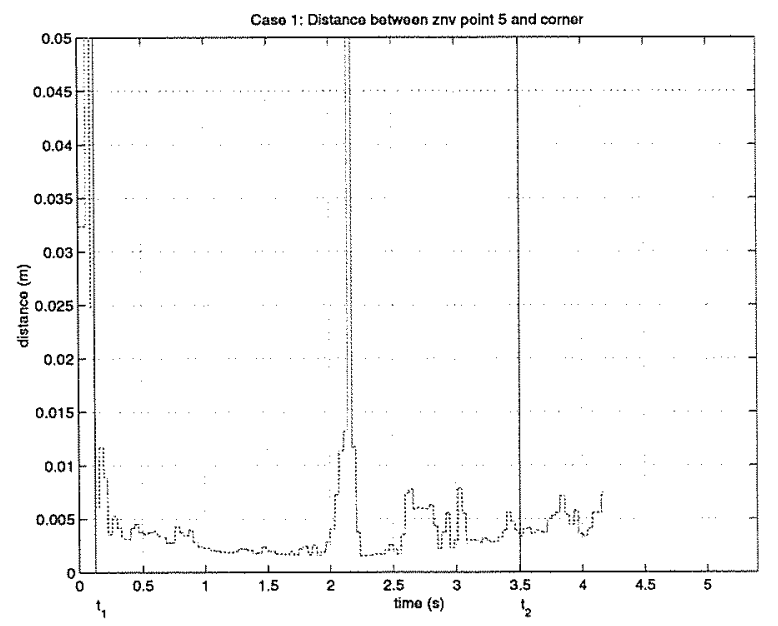

Figure 4-6: Case 1 Experiment: Distance between the computed location of the znv point for edge 5 and the known position of corner c1. During rotation the znv point is seen to track the actual contact point within an accuracy of roughly $\pm 5 \mathrm{~mm}$. The vehicle velocity goes to zero at times $t_{1}$ and $t_{2}$. Very near these times the magnitude of the vehicle velocity is too small to yield reliable readings from the velocity sensors, leading to poor estimates of the znv point location. As soon as the vehicle begins to move again, the error returns to the $\pm 5 \mathrm{~mm}$ range. 


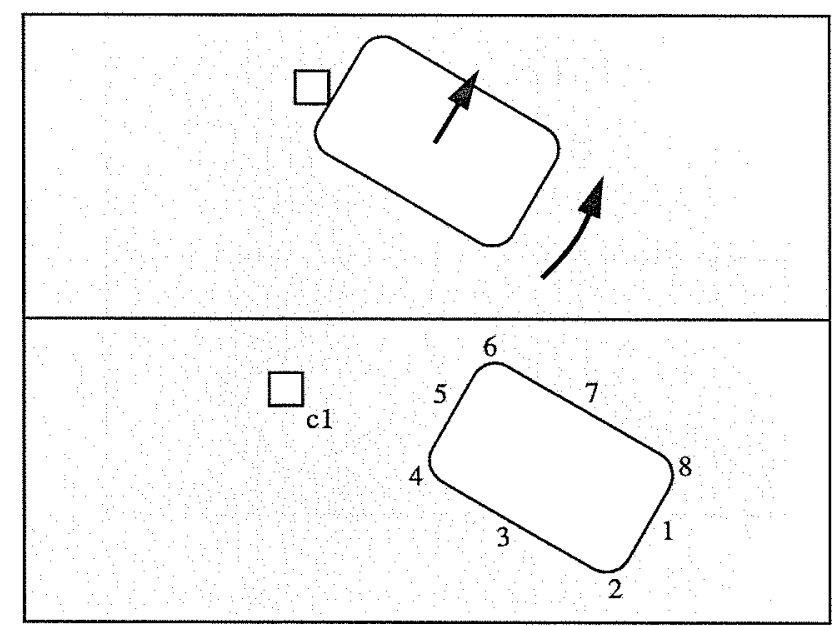

Figure 4-7: Case 2 Experiment: Edge 5 of the vehicle was brought into contact with corner $\mathrm{cl}$ of a stationary object. The vehicle was then moved such that it was in sliding contact with the corner, i.e. the motion combined both rotation and translation of the vehicle relative to the contact point.

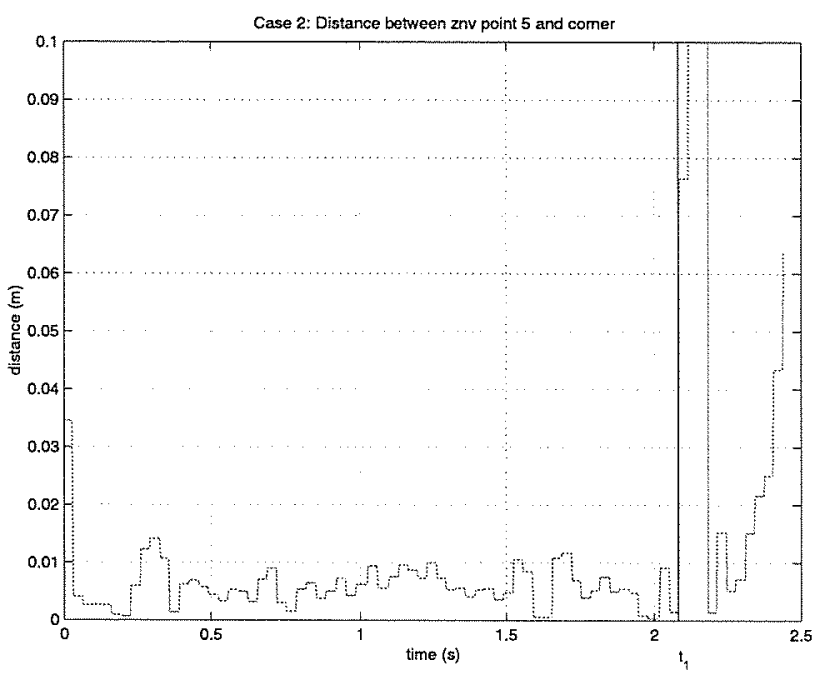

Figure 4-8: Case 2 Experiment: Distance between the computed location of the znv point for edge 5 and the known position of corner $\mathrm{c} 1$. The vehicle was in contact with the corner until time $t_{1}$, at which point contact was broken. The tracking accuracy between the znv point and the known contact location is within $10 \mathrm{~mm}$ for this trial. 


\subsubsection{Case 3: Two Point Contact (Pure Translation)}

In this trial there is no unique contact point to track on edge 5 ; every point on edge 5 is a valid candidate contact point. For such motions the technique presented in this thesis will not be able to identify the actual contact points. The technique will still be able to determine the vehicle's constrained directions however. While edge 5 has no unique candidate contact point, both of its adjacent arc segments do. Furthermore, the candidate contact points for the arcs bound the infinite set of candidate contact points associated with edge 5. The model associated with the two arcs' candidate contact points, i.e. model $(6,7)$, imposes force and velocity constraints identical to those imposed by any model formed from two distinct points lying on edge 5 . Thus, with regard to the constraints, all models formed from points on edge 5 are equivalent, so we can use any one model to represent the set. A natural choice is the model $(6,7)$. We revisit the pure translation case when testing the complete identification system in Chapter 8.

\subsubsection{Case 4: Two Point Contact (Rotation and Translation)}

Figure 4-10 shows the vehicle motion during experiment 4 . Initially vehicle edge 5 is in contact with corner $\mathrm{c} 1$ and vehicle $\operatorname{arc} 4$ is in contact with corner $\mathrm{c} 2$ (we shall refer to this contact configuration as simply $(4,5))$. At time $t=0$ the vehicle begins rotating in a clockwise direction, leading to three transitions in contact configuration;

1. from $(4,5)$ to $(3,5)$ (i.e. $4-10$.a to $4-10$.b ) at time $t_{1}$

2. from $(3,5)$ to $(3,4)$ (i.e. $4-10 . b$ to $4-10$.c ) at time $t_{2}$.

3. from $(3,4)$ to no contact at time $t_{3}$

The upper plot in Figure 4-11 shows the distances between corner $\mathrm{c} 1$ and the znv points for edge 3 , edge 5 and arc 4 during the trial while the lower plot shows the corresponding distances for corner $\mathrm{c} 2$. Prior to the first transition at time $t_{1}$ the 
actual contact configuration is $(4,5)$ and we see from the plots that the znv points for boundary segments edge 5 and arc 4 track the actual contact points quite well. At time $t_{1}$ the contact at corner 22 transitions from boundary segment arc 4 to boundary segment edge 3 , which is clearly reflected by the crossover of the curves for znv points 4 and 3 in the upper plot. Between times $t_{1}$ and $t_{2}$ the znv point for segment edge 3 accurately tracks corner one and znv point for edge 5 accurately tracks corner c2. The second transition occurs at time $t_{2}$, after which we see that the znv point data again agrees well with the actual contact configuration $(3,4)$.

\subsection{Conclusion}

This chapter presented a means to locate the set of kinematically feasible candidate contact points for a moving rigid body. Experiments demonstrated that the positions of the appropriate candidate contact points could track the actual contact point(s) to within 0.01 meters on a 0.145 by 0.290 meter rectangular rigid body. 


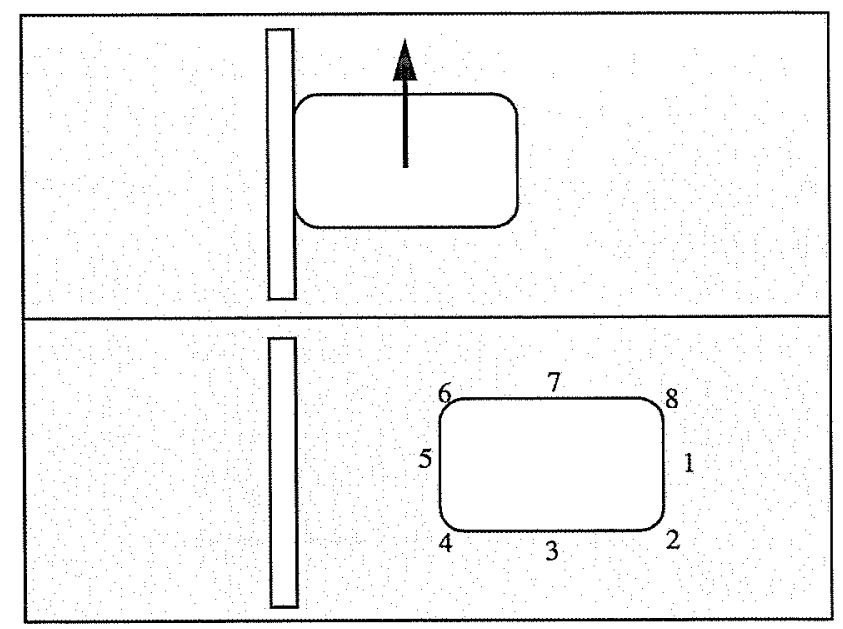

Figure 4-9: Case 3 Experiment: Edge 5 of the vehicle was brought into full contact with a flat wall. This contact was maintained throughout the trial as the vehicle was tran:

The

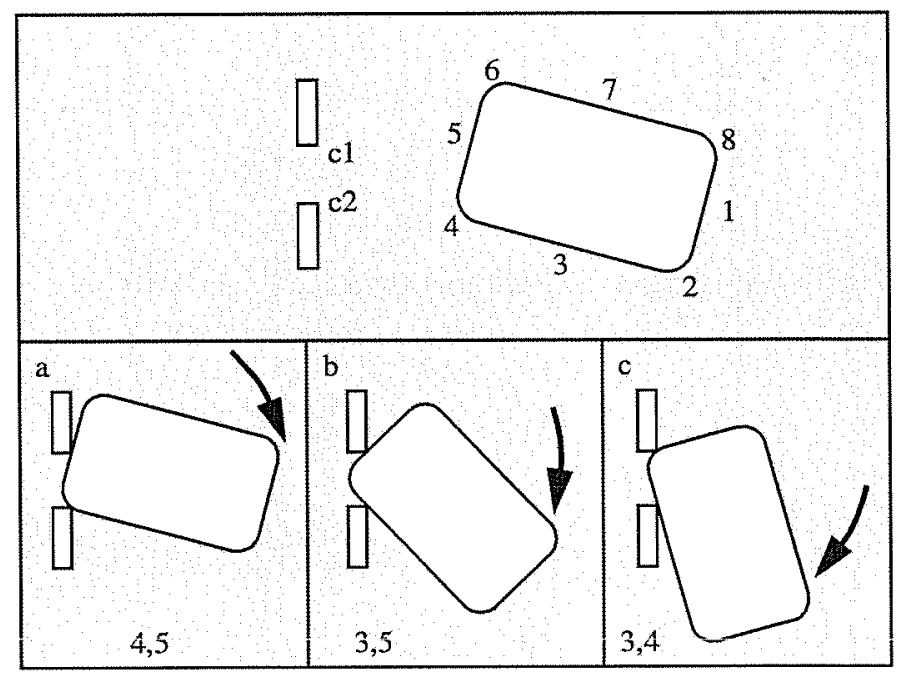

Figure 4-10: Case 4 Experiment: Arc 4 and edge 5 were initially in contact with corners $\mathrm{c} 1$ and $\mathrm{c} 2$ (we refer to this contact state as $(4,5)$ ). 'The vehicle was then moved such that the contact state transitioned to $(3,5)$ and then to $(3,4)$. 

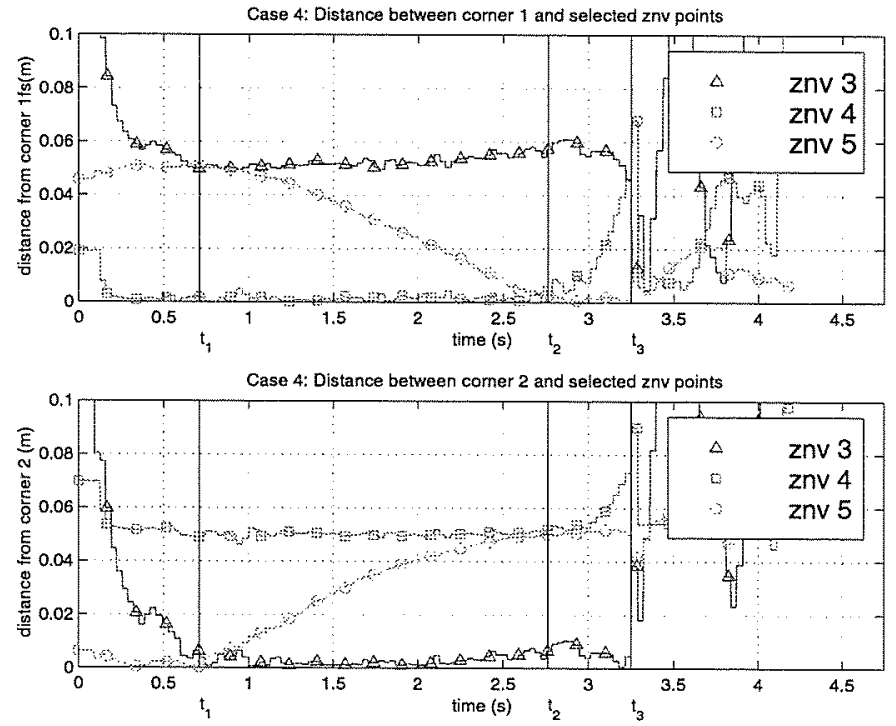

Figure 4-11: Upper plot: Distance between corner c1 and the znv points for edge 3, $\operatorname{arc} 4$ and edge 5. Lower plot: Distance between corner $\mathrm{c} 2$ and the znv points for edge 3 , arc 4 and edge 5 . 


\section{Chapter 5}

\section{Constructing Kinematically Feasible Candidate Contact Models}

\section{$5.1 \quad$ Introduction}

The candidate contact points identified in the previous chapter are the only points on the vehicle which could possibly be in contact with fixed bodies in the environment. This leads to three conclusions:

1. The actual contact points must be members of the set of candidate contact points.

2. The only possible candidate contact models are those constructed from combinations of candidate contact points.

3. The actual contact model must be a member of the set of candidate contact models.

In this chapter we construct the set of candidate contact models, showing that there are five classes of candidate contact models for a planar, rigid body interacting with fixed planar, rigid bodies in its environment. By combining the constraints 
imposed at each of a candidate model's presumed contact points we obtain two sets of constraint equations for each candidate model. The model's force constraints are a set of quasi-static equilibrium equations which relate forces applied to the vehicle at the candidate model's presumed contact points to the corresponding force and moment that would be measured by the vehicle's force sensor. The model's motion constraints are a set of kinematic equations which relate velocities at the model's presumed contact points to the velocity that would be measured by the vehicle velocity sensor. To simplify the derivations we assume throughout this thesis, with no loss of generality, that both the force and velocity sensors are located at the origin of the vehicle fixed reference frame $V$. We follow a general discussion of the characteristics of a model's force and motion constraint equations with detailed derivations of these equations for models in each of the five model classes.

\section{Assumptions}

All of the assumptions made in the introduction to Part II also hold for this chapter.

\subsection{Measuring the Net Contact Induced Force and Moment}

To determine which of the candidate points are actual contact points we will need to measure the net contact force and moment applied to the vehicle as a result of contact. In this section we briefly discuss how this measurement can be performed. Figure 5-1 shows one possible approach to measuring the net force and moment applied to the vehicle as a result of contact with the environment. In the figure three stiff, uniaxial load cells attach a rigid frame to the vehicle. The frame completely surrounds the vehicle, thus only the frame can contact objects in the environment. Since all forces applied to the frame are transmitted to the vehicle via the load cells, we can determine the net force and moment applied to the vehicle from the load cell measurements. 


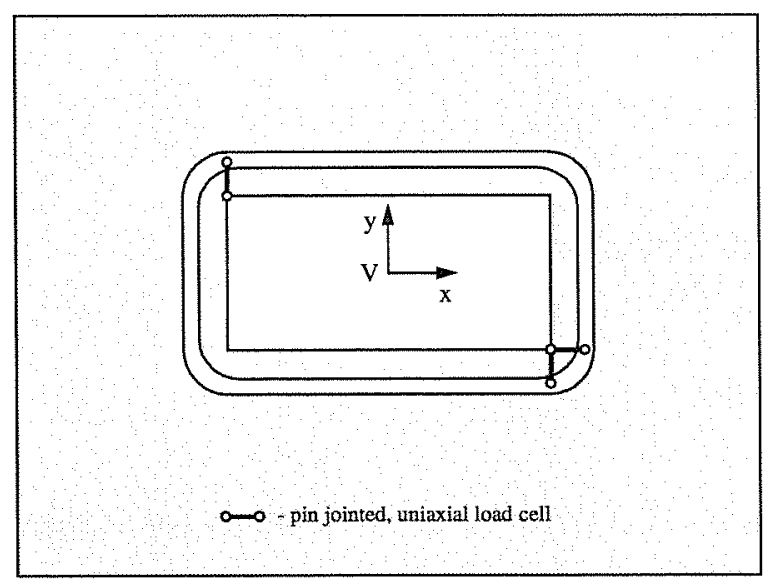

Figure 5-1: Possible design of an instrumented rigid frame to measure the net force and moment applied to the vehicle as a result of contact with objects in the environment. The frame is the only part of the vehicle which can come into contact with the environment. Three pin-jointed, uniaxial force sensors would rigidly affix the frame to the main vehicle structure. The three components of the net force and moment applied to the vehicle $f_{x}, f_{y}$ and $m_{z}$ can be determined from the force measurements from the three load cells.

The design shown is just one possible design. In the planar vehicle apparatus used to perform the experiments presented in this thesis, the frame was actually attached to the vehicle via a commercial 6-axis force-torque sensor ( note: only the $f_{x}, f_{y}$ and $m_{z}$ components of the sensor's output were used ).

For convenience we define the body referenced measured force vector $\mathcal{F}_{m}$

$$
\mathcal{F}_{m}=\left[\begin{array}{c}
f_{x} \\
f_{y} \\
m_{v_{O}}
\end{array}\right]
$$




\begin{tabular}{|c|c|c|c|}
\hline Model Class & $\begin{array}{c}\text { Dim. of } \\
\text { permissible } \\
\text { force space }\end{array}$ & $\begin{array}{c}\text { Dim. of } \\
\text { permissible } \\
\text { vel. space }\end{array}$ & $\begin{array}{c}\text { No. of } \\
\text { models } \\
\text { in class }\end{array}$ \\
\hline Unconstrained & 0 & 3 & 1 \\
\hline One Point, with slip & 1 & 2 & $N$ \\
\hline One Point, no slip & 2 & 1 & $N$ \\
\hline Two Point & 2 & 1 & $\frac{N !}{(N-2) !(2) !}$ \\
\hline Fully Constrained & 3 & 0 & 1 \\
\hline
\end{tabular}

Table 5.1: The five classes of possible contact models for a rigid, planar body interacting with fixed rigid, planar bodies in its environment

Similarly, we define measured velocity vector $\mathcal{V}_{m}$ to be

$$
\mathcal{V}_{m}=\left[\begin{array}{c}
v_{x} \\
v_{y} \\
\omega
\end{array}\right]
$$

The elements of $\mathcal{V}_{m}$ represent the absolute velocity of the origin of the vehicle fixed frame $V$ expressed with respect to an inertial frame which is instantaneously aligned with frame $V$.

\subsection{Model Classes}

In this section we show that there are five classes of possible contact models for a planar rigid body interacting with fixed planar, rigid bodies in its environment. These classes are characterized by two properties; the number of contact points and whether or not slip occurs at each contact point. We also express the number of candidate contact models in each class as a function of the number candidate contact points. The contact model classes are

Unconstrained Model Given $N$ candidate contact points there is one model in which none of the candidate points are actual contact points. In this case the 
vehicle motion is completely unconstrained. There is only one model in this class.

One Point Contact Models with Slip Given $N$ candidate contact points we can formulate $N$ unique models in which the vehicle has a single, sliding point of contact with the environment.

One Point Contact Models with No Slip Given $N$ candidate contact points we can formulate $N$ unique models in which the vehicle has a single, non-sliding point of contact with the environment.

Two Point Contact Models Given $N$ candidate contact points there are $\frac{N !}{(N-2) !(2) !}$ unique pairings of candidate contact points. There are three possible slip states for each pairing ( i.e. neither point slips, one point slips, or both points slip) which implies that there are $\frac{3 N !}{(N-2) !(2) !}$ possible two point contact models. However, the models associated with two of these slip states can be discarded. Models in which neither point slips are actually members of the Fully Constrained Models class listed below. Models in which only one point slips are only possible if the fixed objects in the environment have very specific and extremely precise geometry. In fact, it would be very difficult to purposefully fabricate parts which could realize these contact states. Therefore we also ignore the models associated with this slip state. Thus we consider only the $\frac{N !}{(N-2) !(2) !}$ models in which both points slip.

Fully Constrained Models When the vehicle is fully constrained its linear and angular velocities are zero. The identification technique we develop in this thesis cannot identify the correct contact points when the vehicie is not moving, it cannot distinguish one member of the Fully Constrained Contact Models from another. The best we can do is to correctly identify that the vehicle is fully constrained. Thus we treat the fully constrained models class as a single candidate model. 


\subsection{The Permissible Force Space}

We will find that the force constraint equations for any contact model can be written in the form

$$
\mathcal{F}_{P}=\mathrm{Af}_{c}
$$

where $\mathbf{f}_{c}$ is an $n \times 1$ vector containing $n$ independent parameters which fully describe the forces acting on the vehicle at the model's presumed contact points, $\mathbf{A}$ is a $3 \times n$ matrix containing the coefficients of the force constraint equations and $\mathcal{F}_{P}$ is a $3 \times 1$ vector containing the corresponding force and moment that would be measured by a force sensor located at the origin of frame $V$. The value of $n$ depends on the class of the candidate contact model as reflected in Table 5.1.

Equation 5.3 is a linear mapping from the space of forces $\mathbf{f}_{c} \in R^{n}$ applied at the model's presumed contact points into the space of possible measured forces $\mathcal{F}_{m} \in R^{3}$. This mapping partitions the space of possible measured forces $\mathcal{F}_{m} \in R^{3}$ into two mutually orthogonal subspaces. A model's permissible force space is the $n$ dimensional subspace of possible measured force vectors $\mathcal{F}_{m}$ for which solutions of the model's force constraint equations exist; mathematically, the permissible force space is the column space of A. A model's impermissible force space is the $(3-n)$ dimensional subspace of possible measured force vectors $\mathcal{F}_{m}$ which the candidate contact model is physically incapable of generating; mathematically, the impermissible force space is the left null space of $\mathbf{A}$. Together, these two subspaces span the space of possible measured force vectors $\mathcal{F}_{m}$. Therefore an arbitrary measured force vector $\mathcal{F}_{m}$ can be written as

$$
\mathcal{F}_{m}=\mathcal{F}_{P}+\mathcal{F}_{I}
$$

where $\mathcal{F}_{P}$ is a permissible component which resides wholely within the model's per- 
missible force space and $\mathcal{F}_{I}$ is an impermissible component which resides wholely in the model's impermissible force space.

Equation 5.4 implies that we can decompose the measured force vector $\mathcal{F}_{m}$ into a permissible and impermissible component for each candidate contact model. The magnitude of the resulting impermissible component gives an indication of the the model's compatibility with the observed measurement $\mathcal{F}_{m}$, but, by itself, cannot

identify the correct model. To see this, consider that the measured force vector $\mathcal{F}_{m}$ is not arbitrary; it results from the actual state of contact between the vehicle and the environment and therefore must reside entirely within the permissible force space of the contact model describing this contact state (i.e. the correct contact model). Therefore we expect the decomposition of $\mathcal{F}_{m}$ associated with the correct contact model to yield, in an ideal world, $\mathcal{F}_{I}=\mathbf{0}$. This characteristic, however, is not unique to the correct model; for example, we will find that the impermissible force component for the Fully Constrained model is always equal to zero.

In section 6.2 we show how to decompose $\mathcal{F}_{m}$ into its permissible and impermissible components for each candidate contact model and how to use these components in selecting the correct candidate contact model.

\subsection{The Permissible Velocity Space}

We will find that the velocity constraint equations for each candidate contact model can be written in the form

$$
\mathcal{V}_{P}=\mathrm{Bv}_{c}
$$

where $\mathbf{v}_{c}$ is an $(3-n) \times 1$ vector containing $(3-n)$ independent parameters which fully describe the vehicle's constrained motion under the given model (typically these are velocities at the contact points), $\mathrm{B}$ is a $3 \times(3-n)$ matrix containing the coefficients of the motion constraint equations, $\mathcal{V}_{P}$ is a $3 \times 1$ vector containing the linear 
and angular velocity of the origin of frame $V$ and $n$ is, as defined above, the number of independent parameters required to required to describe the contact point forces in the force constraint equations.

The properties of the velocity constraint equations are largely analogous to those of the force constraint equations only the dimensions of the permissible and impermissible vector spaces are different. Equation 5.5 is a linear mapping from the space of velocities $\mathbf{v}_{c} \in R^{(3-n)}$ at the model's presumed contact points into the corresponding space of measured vehicle velocities $\mathcal{V}_{m} \in R^{3}$. This mapping partitions the space of possible measured velocities $\mathcal{V}_{m} \in R^{3}$ into two mutually orthogonal subspaces. A model's permissible velocity space is the $(3-n)$ dimensional subspace of possible measured velocity vectors $\mathcal{V}_{m}$ for which solutions of the model's velocity constraint equations exist; mathematically, the permissible velocity space is the column space of B. A model's impermissible velocity space is the $n$ dimensional subspace of possible measured velocity vectors $\mathcal{V}_{m}$ which are physically impossible under the given candidate contact model; mathematically, the impermissible velocity space is the left null space of B. Together, these two subspaces span the space of possible measured velocity vectors $\mathcal{V}_{m}$. Therefore an arbitrary measured velocity vector $\mathcal{V}_{m}$ can be written as

$$
\mathcal{V}_{m}=\mathcal{V}_{P}+\mathcal{V}_{I}
$$

where $\mathcal{V}_{P}$ is a permissible component which resides wholely within the model's permissible velocity space and $\mathcal{V}_{I}$ is an impermissible component which resides wholely in the model's impermissible velocity space.

Thus we can decompose the the measured velocity vector $\boldsymbol{V}_{m}$ into permissible and impermissible components for each candidate contact model. Were we to do so, however, we would discover that the measured velocity vector $\mathcal{V}_{m}$ exactly satisfies the velocity constraint equations of every candidate contact model. Recall that we found the candidate contact points by solving for points on the vehicle boundary whose 
individual contact constraints were exactly consistent with the measured vehicle velocity $\mathcal{V}_{m}$. We derive the velocity constraint equations by combining the velocity constraints associated with each candidate contact model's candidate contact points. Since $\mathcal{V}_{m}$ exactly satisfies the constraints at every candidate contact point, it also exactly satisfies every candidate contact model's velocity constraint equations,i.e.

$$
\mathcal{V}_{I}=\mathbf{0}
$$

Thus we also have

$$
\mathcal{V}_{P}=\mathcal{V}_{m}
$$

This characteristic is a dual edged sword; it will greatly simplify the process of solving for each model's reaction loads and in decomposing the measured force into its permissible and impermissible components in Chapter 6. However, this same property prevents the technique presented in that chapter from identifying the best overall model (instead, it can only identify the best model in each class).

In Chapter 7 we overcome this limitation by also considering the incremental motion of the vehicle. We consider the constraints on incremental motion of the vehicle in the following section.

\subsection{The Permissible Motion Space}

If we multiply both sides of the velocity constraint equations 5.5 by a differential length of time $d t$ we obtain the differential motion constraint equations ( referred to hereafter as the motion constraint equations) for the vehicle, i.e.

$$
\delta \mathcal{X}_{P}=\mathbf{B} \delta \mathbf{x}_{c}
$$


where $\delta \mathrm{x}_{c}$ is an $(3-n) \times 1$ vector containing $(3-n)$ independent parameters characterizing the differential motion of the vehicle's presumed contact points, $B$ is the same $3 \times(3-n)$ matrix used in the velocity constraint equations above, $\delta \mathcal{X}_{P}$ is a $3 \times 1$ vector containing the differential motion of the origin of frame $V$, and $n$ is, as defined above, the number of independent parameters required to describe the contact point forces in the force constraint equations. Obviously the differential motion constraints share all of the same properties of the velocity constraints, i.e. their permissible and impermissible spaces are identical except for the units.

We cannot measure the differential motion of the vehicle directly, however; we can only approximate it using current and previous measurements of the vehicle position vector, i.e. we can define the vehicle's incremental motion vector $\Delta \boldsymbol{X}_{m}$ to be

$$
\Delta \mathcal{X}_{m}=\mathcal{X}_{m_{i}}-\mathcal{X}_{m_{i-k}}
$$

where $\boldsymbol{X}_{m_{i}}$ is the vehicle's current measured position vector and $\boldsymbol{X}_{m_{i-k}}$ a previous measured position vector from $k$ time steps in the past. Replacing the differential motions $\delta \mathcal{X}_{P}$ and $\delta \mathbf{x}_{c}$ with their incremental counterparts $\Delta \mathcal{X}_{P}$ and $\Delta \mathrm{x}_{c}$ equation 5.9 becomes

$$
\Delta \mathcal{X}_{P}=\mathbf{B} \Delta \mathbf{x}_{c}
$$

As was the case with the velocity vector, we can decompose the incremental motion vector into permissible and impermissible components, i.e.

$$
\Delta \mathcal{X}_{m}=\Delta \mathcal{X}_{P}+\Delta \mathcal{X}_{I}
$$

Unlike the velocity decomposition, however, $\Delta \mathcal{X}_{m}$ will not, in general, exactly satisfy the constraint equations. Consequently $\Delta \mathcal{X}_{I}$ is not inherently equal to zero. Thus using the impermissible component of the incremental motion vector $\Delta \boldsymbol{X}_{m}$ 
allows us to estimate the degree to which the vehicle's motion in the very recent past is consistent with the differential motion constraints.

\subsection{Model Constraint Equations}

In the previous sections we described the general characteristics of a candidate contact model based on its force and velocity constraint equations. In this section we derive these constraint equations for each class of candidate contact model.

\subsubsection{Unconstrained Model}

\section{Force Constraint Equations}

When fully unconstrained the vehicle makes no contact with the environment. Therefore the force constraint equations for the unconstrained model are

$$
\mathcal{F}_{P}=\left[\begin{array}{l}
0 \\
0 \\
0
\end{array}\right]
$$

The permissible force space is a single point, that being the origin of the three dimensional space of possible vectors $\mathcal{F}_{P}$. Therefore the model requires $n=0$ independent parameters to describe the (nonexistent) applied loads.

\section{Motion Constraint Equations}

Any vehicle velocity is permissible under the unconstrained model. Specifying this velocity requires $n=3$ independent parameters. Since the model has no contact points, and therefore no contact point velocities, we select the elements of the vector $\mathcal{V}_{m}$ as our three independent velocity parameters. The velocity constraint equations 


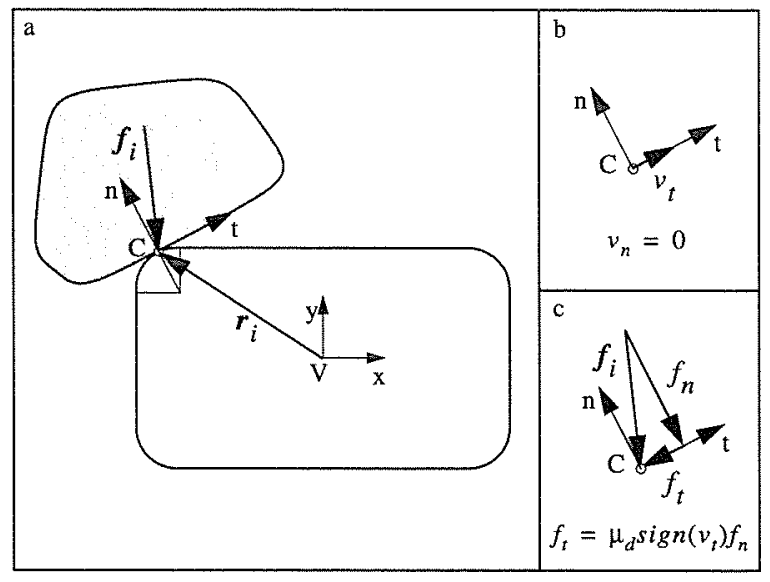

Figure 5-2: (a) Vehicle with a single, sliding point of contact with a fixed rigid body in the environment. (b) Absolute velocity of the point on the vehicle which is in contact in contact frame coordinates. (c) Forces acting at the contact point in contact frame coordinates. When the contact involves sliding, the tangential component $f_{t}=\mu_{d} \operatorname{sign}\left(v_{t}\right) f_{n}$, so describing the contact force requires only one independent parameter: $f_{n}$.

for the unconstrained model are then

$$
\mathcal{V}_{P}=\left[\begin{array}{lll}
1 & 0 & 0 \\
0 & 1 & 0 \\
0 & 0 & 1
\end{array}\right] \mathcal{V}_{m}
$$

i.e matrix $\mathbf{B}$ is equal to the $3 \times 3$ identity matrix.

\subsubsection{One Point Contact Models with Slip}

Figure 5-2 shows the vehicle with a single, sliding point of contact with a fixed rigid body. The contact occurs at candidate contact point $i$ whose position, relative to the vehicle fixed reference frame $V$, is given by vector $\mathbf{r}_{i}$. 


\section{Force Constraint Equations}

Assuming a Coulomb model for the friction force that acts at the contact point, the tangential and normal components of the contact force $\mathbf{f}_{i}$ are related by the following equality

$$
f_{t_{i}}=\mu_{d} \operatorname{sign}\left(v_{t_{i}}\right) f_{n_{i}}
$$

where $\mu_{d} \geq 0$ is the (known) dynamic coefficient of friction and $v_{t_{i}}$ is the (known) tangential component of the absolute velocity of the candidate contact point which we compute from the vehicle velocity and geometry. Note that the friction force $f_{t_{i}}$ applied to the vehicle acts in the opposite direction of the tangential velocity $v_{t_{i}}$ for contact forces having feasible normal components (i.e. for contact forces where $\left.f_{n_{i}}<0\right)$.

Because the contact force passes through the contact point, it induces no moment about that point. Relative to the contact frame $C_{i}$ the contact force is

$$
\mathcal{F}_{C}=\left[\begin{array}{c}
f_{t_{i}} \\
f_{n_{i}} \\
m_{C}
\end{array}\right]_{C}=\left[\begin{array}{c}
\mathbf{u}_{f_{i}} \\
0
\end{array}\right] f_{n_{i}}
$$

where

$$
\mathbf{u}_{f_{i}}=\left[\begin{array}{c}
\mu_{d} \operatorname{sign}\left(v_{t_{i}}\right) \\
1
\end{array}\right]
$$

gives the direction of the force $\mathbf{f}_{i}$ applied to the vehicle at the contact point (note, however, that $\mathbf{u}_{f_{i}}$ is not a unit vector). Thus the One Contact Point with Slip Models require $n=1$ independent parameter, $f_{n_{i}}$, to fully describe the loads applied at the contact point. 
Relating the applied force and moment $\mathcal{F}_{C}$ to the force and moment $\mathcal{F}_{P}$ experienced by the force sensor we obtain the force constraint equations for the One Point Contact with Slip models

$$
\mathcal{F}_{P}=\left[\begin{array}{c}
f_{x} \\
f_{y} \\
m_{V_{O}}
\end{array}\right]_{V}=\left[\begin{array}{c}
\mathbf{R}_{i} \mathbf{u}_{f_{i}} \\
{\left[\mathbf{r}_{i} \times\right]^{T} \mathbf{R}_{i} \mathbf{u}_{f_{i}}}
\end{array}\right] f_{n_{i}}
$$

where $\mathbf{R}_{i}$ is the $2 \times 2$ rotation matrix which transforms vectors from the contact frame $C_{i}$ into the sensor frame $V$ (recall that the sensor frame is coincident with the vehicle frame $V), \mathbf{r}_{i}$ is a $2 \times 1$ vector giving the position of candidate contact point $i$ relative to the vehicle frame $V$ and

$$
\left[\mathbf{r}_{i} \times\right]=\left[\begin{array}{c}
-r_{i_{y}} \\
r_{i_{x}}
\end{array}\right]
$$

as previously defined (see Appendix I for a complete description of this notation).

Comparing equation 5.18 to equation 5.3 we see that

$$
\begin{aligned}
\mathbf{A} & =\left[\begin{array}{c}
\mathbf{R}_{i} \mathbf{u}_{f_{i}} \\
{\left[\mathbf{r}_{i} \times\right]^{T} \mathbf{R}_{i} \mathbf{u}_{f_{i}}}
\end{array}\right] \\
\mathbf{f}_{c} & =\left[f_{n_{i}}\right]
\end{aligned}
$$

\section{Motion Constraint Equations}

The velocity of candidate contact point $i$ in contact frame coordinates can be written as

$$
\mathcal{V}_{C}=\left[\begin{array}{c}
v_{t_{i}} \\
v_{n_{i}} \\
\omega
\end{array}\right]=\left[\begin{array}{cc}
\mathbf{u}_{v_{t_{i}}} & 0 \\
0 & 1
\end{array}\right]\left[\begin{array}{l}
v_{t_{i}} \\
\omega
\end{array}\right]
$$


where $\omega$ is the vehicle's angular velocity and

$$
\mathbf{u}_{v_{t_{i}}}=\left[\begin{array}{l}
1 \\
0
\end{array}\right]
$$

We obtain the motion constraint equations for this class of models by relating the contact point velocity $\mathcal{V}_{C_{O}}$ to the velocity $\mathcal{V}_{P}$ experienced by the velocity sensor

$$
\mathcal{V}_{P}=\left[\begin{array}{c}
v_{x} \\
v_{y} \\
\omega
\end{array}\right]_{V}=\left[\begin{array}{cc}
\mathbf{R}_{i} \mathbf{u}_{v_{t_{i}}} & -\left[\mathbf{r}_{i} \times\right] \\
0 & 1
\end{array}\right]\left[\begin{array}{c}
v_{t_{i}} \\
\omega
\end{array}\right]
$$

Comparing equation 5.24 to equation 5.5 we see that

$$
\begin{aligned}
& \mathbf{B}=\left[\begin{array}{cc}
\mathbf{R}_{i} \mathbf{u}_{v_{t_{i}}} & -\left[\mathbf{r}_{i} \times\right] \\
0 & 1
\end{array}\right] \\
& \mathbf{v}_{c}=\left[\begin{array}{c}
v_{t_{i}} \\
\omega
\end{array}\right]
\end{aligned}
$$

\subsubsection{One Point Contact Models with No Slip}

Figure 5-3.a shows the case of a vehicle in single point contact with the environment where no slip occurs at the contact point, i.e. the absolute velocity of the point on the vehicle which is in contact is zero. The contact occurs at candidate contact point $i$ whose position, relative to the vehicle fixed reference frame $V$, is given by vector $\mathbf{r}_{i}$.

\section{Force Constraint Equations}

For the contact scenario shown, static friction is the only mechanism which could so constrain the contact point. In this case the tangential and normal components of the contact force are no longer related by an equality but by the following inequality 


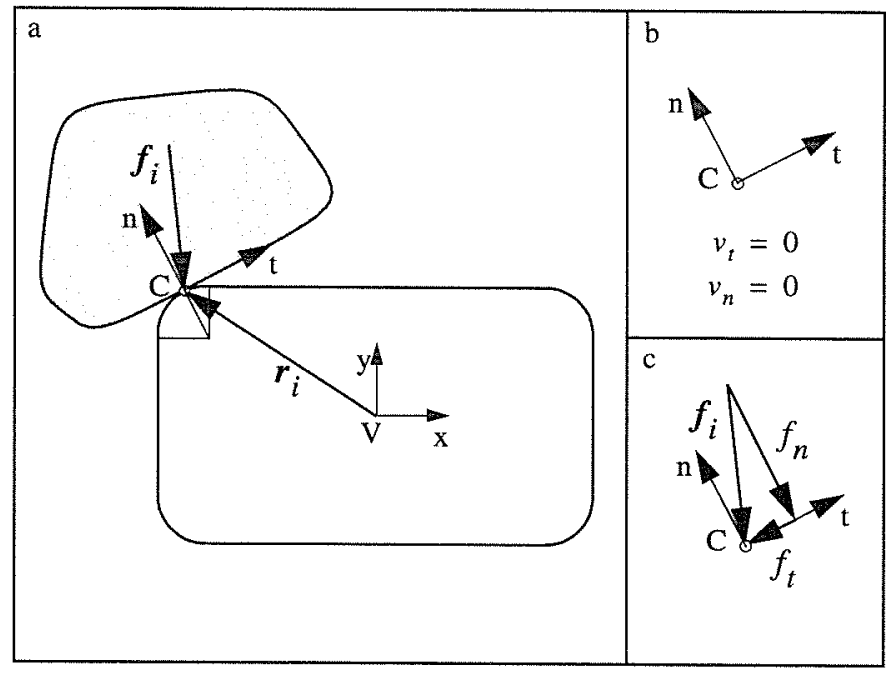

Figure 5-3: (a) Vehicle with a single, non-sliding point of contact with a fixed rigid body in the environment. (b) Absolute velocity of the point on the vehicle which is in contact in contact frame coordinates. (c) Forces acting at the contact point in contact frame coordinates. When the contact point is non-sliding, the tangential and normal components $f_{t}$ and $f_{n}$ are independent parameters.

$$
\left|f_{t_{i}}\right| \leq \mu_{s}\left|f_{n_{i}}\right|
$$

where $\mu_{s}$ is the (unknown) static coefficient of friction between the vehicle and the fixed body. As long as the tangential velocity is zero we must conclude that equation 5.27 is satisfied, in which case $f_{t_{i}}$ and $f_{n_{i}}$ are independent variables.

Again, since the contact force passes through the origin of the contact frame it induces no moment about that point. Thus we can write the contact force in contact frame $C_{i}$ coordinates as

$$
\mathcal{F}_{C}=\left[\begin{array}{c}
f_{t_{i}} \\
f_{n_{i}} \\
m_{C}
\end{array}\right]_{C}=\left[\begin{array}{cc}
\mathbf{u}_{f_{t_{i}}} & \mathbf{u}_{f_{n_{i}}} \\
0 & 0
\end{array}\right]\left[\begin{array}{l}
f_{t_{i}} \\
f_{n_{i}}
\end{array}\right]
$$


where

$$
\mathbf{u}_{f_{t_{i}}}=\left[\begin{array}{l}
1 \\
0
\end{array}\right], \quad \mathbf{u}_{f_{n_{i}}}=\left[\begin{array}{l}
0 \\
1
\end{array}\right]
$$

Thus the One Contact Point with No Slip Models require $n=2$ independent parameters, $f_{t_{i}}$ and $f_{n_{i}}$, to fully describe the load applied at the contact point.

Relating the applied force and moment $\mathcal{F}_{C}$ to the force and moment $\mathcal{F}_{P}$ experienced by the force sensor we obtain the force constraint equations for the One Point Contact with No Slip models

$$
\mathcal{F}_{P}=\left[\begin{array}{c}
f_{x} \\
f_{y} \\
m_{V}
\end{array}\right]_{V}=\left[\begin{array}{c}
\mathbf{R}_{i} \\
{\left[\mathbf{r}_{i} \times\right]^{T} \mathbf{R}_{i} \mathbf{u}_{f_{t_{i}}}}
\end{array}\left[\begin{array}{c}
\left.\mathbf{r}_{i} \times\right]^{T} \mathbf{R}_{i} \mathbf{u}_{f_{n_{i}}}
\end{array}\right]\left[\begin{array}{c}
f_{t_{i}} \\
f_{n_{i}}
\end{array}\right]\right.
$$

Comparing equation 5.29 to equation 5.3 we see that

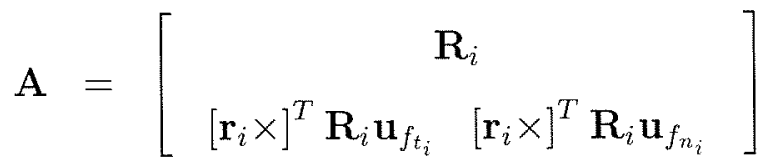

$$
\begin{aligned}
& \mathbf{f}_{c}=\left[\begin{array}{l}
f_{t_{i}} \\
f_{n_{i}}
\end{array}\right]
\end{aligned}
$$

\section{Motion Constraint Equations}

In any One Point Contact with No Slip model the vehicle undergoes pure rotation about the model's candidate contact point. In this case we can write the motion constraint equations directly as 


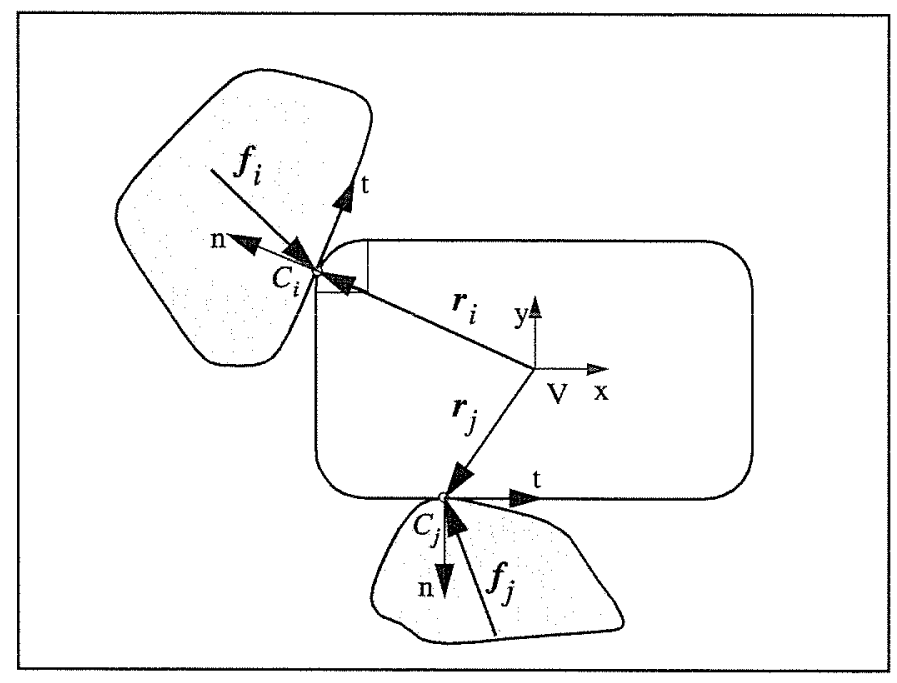

Figure 5-4: Vehicle with a two sliding points of contact with a fixed rigid bodies in the environment. Since sliding occurs at both contacts, the tangential components of the contact forces are $f_{t_{i}}=\mu_{d} \operatorname{sign}\left(v_{t_{i}}\right) f_{n_{i}}$ and $f_{t_{j}}=\mu_{d} \operatorname{sign}\left(v_{t_{j}}\right) f_{n_{j}}$, i.e. describing the contact forces requires two independent parameter: $f_{n_{i}}$ and $f_{n_{j}}$.

$$
\mathcal{V}_{P}=\left[\begin{array}{c}
v_{x} \\
v_{y} \\
\omega
\end{array}\right]_{V}=\left[\begin{array}{c}
-\left[\mathbf{r}_{i} \times\right] \\
1
\end{array}\right] \omega
$$

Comparing equation 5.32 to equation 5.5 we see that

$$
\begin{aligned}
& \mathbf{B}=\left[\begin{array}{c}
-\left[\mathbf{r}_{i} \times\right] \\
1
\end{array}\right] \\
& \mathbf{f}_{c}=[\omega]
\end{aligned}
$$

\subsubsection{Two Point Contact Models}

Figure 5-4.a shows the case of a vehicle in two point contact with the environment where slip occurs at both points of contact. The contacts occur at candidate contact 
points $i$ and $j$ whose positions, relative to the vehicle fixed reference frame $V$, are given by vectors $\mathbf{r}_{i}$ and $\mathbf{r}_{j}$.

\section{Force Constraint Equations}

Based on our analysis of the One Contact Point with Slip models, we can write the contact forces $\mathbf{u}_{f_{i}}$ and $\mathbf{u}_{f_{j}}$ acting at candidate contact points $i$ and $j$ as

$$
\begin{aligned}
\mathcal{F}_{C_{i}}=\left[\begin{array}{c}
f_{t_{i}} \\
f_{n_{i}} \\
m_{C_{O i}}
\end{array}\right]_{C_{i}}=\left[\begin{array}{c}
\mathbf{u}_{f_{i}} \\
0
\end{array}\right] f_{n_{i}} \\
\mathcal{F}_{C_{j}}=\left[\begin{array}{c}
f_{t_{j}} \\
f_{n_{j}} \\
m_{C_{O j}}
\end{array}\right]_{C_{j}}=\left[\begin{array}{c}
\mathbf{u}_{f_{j}} \\
0
\end{array}\right] f_{n_{j}}
\end{aligned}
$$

where

$$
\mathbf{u}_{f_{i}}=\left[\begin{array}{c}
\mu_{d} \operatorname{sign}\left(v_{t_{i}}\right) \\
1
\end{array}\right], \quad \mathbf{u}_{f_{j}}=\left[\begin{array}{c}
\mu_{d} \operatorname{sign}\left(v_{t_{j}}\right) \\
1
\end{array}\right]
$$

To obtain the net force and moment $\mathcal{F}_{P}$ experienced by the force sensor we sum the effects of the two contact forces. Thus the force constraint equations for the Two Contact Point models can be written as

$$
\left[\begin{array}{c}
f_{x} \\
f_{y} \\
m_{V}
\end{array}\right]_{V}=\left[\begin{array}{cc}
\mathbf{R}_{i} \mathbf{u}_{f_{i}} & \mathbf{R}_{j} \mathbf{u}_{f_{j}} \\
{\left[\mathbf{r}_{i} \times\right]^{T} \mathbf{R}_{i} \mathbf{u}_{f_{i}}} & {\left[\mathbf{r}_{i} \times\right]^{T} \mathbf{R}_{j} \mathbf{u}_{f_{j}}}
\end{array}\right]\left[\begin{array}{c}
f_{n_{i}} \\
f_{n_{j}}
\end{array}\right]
$$


Comparing equation 5.38 to equation 5.3 we see that

$$
\begin{aligned}
\mathbf{A} & =\left[\begin{array}{cc}
\mathbf{R}_{i} \mathbf{u}_{f_{i}} & \mathbf{R}_{j} \mathbf{u}_{f_{j}} \\
{\left[\mathbf{r}_{i} \times\right]^{T} \mathbf{R}_{i} \mathbf{u}_{f_{i}}} & {\left[\mathbf{r}_{i} \times\right]^{T} \mathbf{R}_{j} \mathbf{u}_{f_{j}}}
\end{array}\right] \\
\mathbf{f}_{c} & =\left[\begin{array}{c}
f_{n_{i}} \\
f_{n_{j}}
\end{array}\right]
\end{aligned}
$$

\section{Motion Constraint Equations}

The velocity of candidate contact point $i$ in contact frame coordinates can be written as

$$
\mathcal{V}_{C_{O i}}=\left[\begin{array}{c}
v_{t_{i}} \\
v_{n_{i}} \\
\omega
\end{array}\right]=\left[\begin{array}{c}
\mathbf{u}_{v_{t_{i}}} \\
0
\end{array}\right]\left[v_{t_{i}}\right]
$$

where $\omega$ is the vehicle's angular velocity and

$$
\mathbf{u}_{v_{i}}=\left[\begin{array}{l}
1 \\
0
\end{array}\right]
$$

We obtain the motion constraint equations for this class of models by relating the contact point velocity $\mathcal{V}_{C_{O i}}$ to the velocity $\mathcal{V}_{P}$ experienced by the velocity sensor

$$
\mathcal{V}_{P}=\left[\begin{array}{c}
\mathbf{R}_{i} \mathbf{u}_{v_{i}}-\left[\mathbf{r}_{i} \times\right] \frac{1}{\left|\mathbf{r}_{i}\right|} \\
\frac{1}{\left|\mathbf{r}_{i}\right|}
\end{array}\right] v_{t_{i}}
$$

Comparing equation 5.43 to equation 5.5 we see that

$$
\begin{aligned}
& \mathbf{B}=\left[\begin{array}{c}
\mathbf{R}_{i} \mathbf{u}_{v_{i}}-\left[\mathbf{r}_{i} \times\right] \frac{1}{\left|\mathbf{r}_{i}\right|} \\
\frac{1}{\left|\mathbf{r}_{i}\right|}
\end{array}\right] \\
& \mathbf{f}_{c}=\left[v_{t_{i}}\right]
\end{aligned}
$$




\subsubsection{Fully Constrained Model}

Any measured force and moment $\mathcal{F}_{m}$ are permissible under the fully constrained model. Specifying this force and moment requires $n=3$ independent parameters. Since the model has no contact points ( and therefore no contact point velocities), we select the elements of the vector $\mathcal{F}_{m}$ as our three independent force parameters. The force constraint equations for the fully constrained model are then

$$
\mathcal{F}_{P}=\left[\begin{array}{ccc}
1 & 0 & 0 \\
0 & 1 & 0 \\
0 & 0 & 1
\end{array}\right] \mathcal{F}_{m}
$$

i.e matrix $A$ is equal to the $3 \times 3$ identity matrix.

\section{Motion Constraint Equations}

When fully constrained the vehicle linear and angular velocity are zero. Therefore the motion constraint equations for the fully constrained model are

$$
\mathcal{V}_{P}=\left[\begin{array}{l}
0 \\
0 \\
0
\end{array}\right]
$$

i.e. the permissible velocity space is a single point, that being the origin of the three dimensional space of possible vectors $\mathcal{V}_{P}$.

\subsection{Conclusion}

This chapter identified the five classes of planar rigid body contact models and determined, given the number of candidate contact points, the number of candidate contact models that must be evaluated in each identification cycle. We derived the 
force and velocity constraint equations for models in each class and showed that each model's constraint equations define a permissible force and permissible velocity space for the model. In the following chapter we use these vector spaces to decompose the measured force and velocity vectors into permissible and impermissible components for each model. Using these components we will determine the best candidate contact model within each class. 


\section{Chapter 6}

\section{Identifying the Best Candidate Contact Model in Each Class}

\subsection{Introduction}

The set of candidate contact points we identified in Chapter 4 are the only points on the vehicle perimeter which, from a kinematic perspective, could possibly be in contact with the environment. In Chapter 5 we used these points to construct the set of candidate contact models, i.e. the set of all possible contact models which involve the candidate contact points. Thus the correct contact model must be a member of this set.

In this chapter we attempt to identify the the actual model from the set of candidate models using only the instantaneous force and velocity measurements $\mathcal{F}_{m}$ and $\mathcal{V}_{m}$. We will find that this approach can identify the best candidate model within each class, but it cannot identify which of these best-of-class models is the best overall model (In Chapter 7 we show how to determine which of the best-of-class models is the best overall model). In both cases we select the best candidate contact model based on two criteria, feasibility and consistency, which we will define below. The basic approach is simple; test each model for feasibility, evaluate each of the feasible 
models for consistency and then pick the most consistent, feasible model.

\subsubsection{Model Feasibility}

There are two aspects to a model's feasibility. The first is kinematic feasibility which we have already addressed in the previous two chapters. Models involving contact on a given vehicle boundary segment are feasible if and only if the segment's zero normal velocity point falls within the domain of the boundary segment. Thus we enforce kinematic feasibility by only constructing models involving boundary segments which satisfy this condition.

To determine a model's quasistatic feasibility we must first solve for the quasistatic reaction loads at each of the models presumed contact points. In Chapter 4 we noted that rigid bodies in contact can only push against each other, they cannot pull. Mathematically this means that the contact force experienced by a rigid body must have a negative component in the direction of the body's outward facing normal at the contact point, i.e

$$
\mathbf{f}_{r_{i}} \cdot \mathbf{n}_{i}<0
$$

where $\mathbf{f}_{r_{i}}$ is the computed reaction load at the model's $i$ 'th presumed contact point and $\mathbf{n}_{i}$ is the outward facing normal of the vehicle at that point. Thus a model is quasistatically feasible if and only if the quasistatically computed contact forces at each of the models presumed contact points satisfy this condition. Infeasible models are discarded from further consideration. Section 6.2 describes in detail how to solve for the reaction loads for the remaining models in each model class.

\subsubsection{Model Consistency}

The vehicle's measured data result from the physical interactions taking place at the actual contact points. Therefore, in an ideal world, we expect the measured force 
and velocity to be perfectly consistent with (i.e. to exactly satisfy) the force and velocity constraints of the contact model describing the actual contact state. Since this model is necessarily a member of the set of candidate contact models, we can use each model's inconsistency (i.e. the degree to which the measured data violates the model's constraint equations) to identify the correct model.

As shown in the previous chapter, each model has six constraint equations; three force constraints and three velocity constraints. These two sets of constraint equations define the model's permissible force and permissible velocity spaces, respectively. We showed that we can decompose each of the vehicle's measured force and velocity vectors $\mathcal{F}_{m}$ and $\mathcal{V}_{m}$ into the sum of a permissible $\left(\mathcal{F}_{P}\right.$ and $\mathcal{V}_{P}$, respectively) and an impermissible component $\left(\mathcal{F}_{I}\right.$ and $\mathcal{V}_{I}$, respectively) for each candidate contact model. The elements of $\mathcal{F}_{I}$ indicate the degree to which the measured force $\mathcal{F}_{m}$ violates each individual force constraint equation, while the elements of $\mathcal{V}_{I}$ indicate the degree to which the measured velocity $\mathcal{V}_{m}$ violates each individual velocity constraint equation. To evaluate a model's consistency we must construct a consistency measure, defined as a single valued scalar function of the elements of the impermissible components of the measured data vectors. The impermissible components for any of our candidate contact models are

$$
\begin{aligned}
& \mathcal{F}_{I}=\mathcal{F}_{m}-\mathbf{A f}_{c} \\
& \mathcal{V}_{I}=0
\end{aligned}
$$

where we have combined equations 5.3 and 5.4 to get $\mathcal{F}_{I}$ and we obtained $\mathcal{V}_{I}$ from equation 5.7 (recall from Chapter 5 that, because of the way we selected the candidate contact points, the measured velocity $\hat{\mathcal{V}}_{m}$ inherently satisfies the velocity constraints of every candidate model, hence $\mathcal{V}_{I}=0$ ). Thus, for instantaneous data, the consistency measure depends only on violations of a model's force constraint equations.

We face the following challenges in constructing a viable consistency measure: 
Dimensional Consistency Each model has six constraint equations (three force constraint equations and three motion constraint equations) which have four fundamentally different sets of units (i.e. force, moment, linear velocity and angular velocity). To combine these terms into a dimensionally sensible single valued function we must perform some form of dimensional scaling.

Relative Weighting Since the violation terms have different units, each scale factor must have a different magnitude. In effect, scaling is equivalent to weighting the contribution from each equation. We must be very careful to choose a scaling which not only achieves dimensional consistency but also imposes physically justifiable relative weighting to the different constraint equations.

Positive Definiteness Our goal is to select the candidate contact model which is minimally inconsistent with the measured data. Ideally our consistency measure (or, more accurately, our inconsistency measure) should be a positive definite function of the constraint violation terms (i.e. the elements of $\mathcal{F}_{I}$ and $\mathcal{V}_{I}$ ). A positive definite measure will have a unique minimum of zero which occurs if and only if all of the elements of $\mathcal{F}_{I}$ and $\mathcal{V}_{I}$ are zero. Thus the model which has the smallest inconsistency measure is guaranteed to be the most consistent model.

Uniqueness It is possible that different contact models could be equally consistent with a given set of measured data, i.e. it is possible for models to be equivalent. Thus having the smallest inconsistency measure is a necessary but not sufficient condition for a candidate model to be the actual model. One path to equivalency is for two models to yield the same $\mathcal{F}_{I}$ and $\mathcal{V}_{I}$, which is possible only if their permissible force spaces are identical and if they're permissible velocity spaces are also identical. Whether this condition is true or not is independent of the structure of our consistency measure. It is also possible, however, for two models which yield distinct $\mathcal{F}_{I}$ and $\mathcal{V}_{I}$ to produce identical consistency measure values. 
Whether this occurs depends both on the structure of the measure and on the permissible vector spaces of each candidate contact model.

\subsubsection{Approach}

We begin by trying to solve for the reaction loads at each candidate contact model's presumed contact points. To do this we must solve each model's force constraint equations which, for most candidate contact models, are a set of over determined equations. Such equations are generally solved using the method of least squares in which we minimize the length of an error vector (in this case, the length of $\mathcal{F}_{I}$ ). The length of $\mathcal{F}_{I}$, however, is undefined due to the fact that it's elements do not all have the same units. Thus we find that our consistency measure serves not only to choose between candidate models but also plays an integral role in solving for each models reaction loads and, therefore, in determining the feasibility of each model. We introduce the concept of the violation power as a basis for a suitable consistency measure and then use this to resolve the units problem in the original least squares approach to solving for the reaction loads. Once we know a model's reaction loads, we can determine its quasistatic feasibility and we can also compute the permissible and impermissible components of the measured force vector. From these we compute the value of the consistency measure for each model and we explain why the results can only be used to pick the best model within each class of contact models. Finally, we discuss the uniqueness of the best model in each class.

\subsection{Contact Point Reaction Loads}

We find a model's contact point reaction loads by solving the model's force constraint equations. Given the measured force vector $\mathcal{F}_{m}$, we seek a solution vector of unknown 
reaction loads $\mathrm{f}_{c}$ such that

$$
\mathcal{F}_{I}=\mathcal{F}_{m}-\mathrm{Af}_{c}
$$

yields $\mathcal{F}_{I}=0$.

\subsubsection{Problems with a Direct Least Squares Solution for $\mathrm{f}_{c}$}

When $\mathbf{A}$ is not square (i.e. for One Point Contact and Two Point Contact models) there will, in general, be no solution vector $\mathbf{f}_{c}$ for which $\mathcal{F}_{I}=\mathbf{0}$ for a given measured force vector $\mathcal{F}_{m}$. Therefore we will try to find the solution which comes closest to exactly satisying the equations. To do so we form a positive definite, single valued error metric $r$ which is zero if and only the solution $\mathrm{f}_{c}$ exactly satisfies equations 6.4 and then select the solution which minimizes $r$. The most common error metric is the square of the length of the error vector $\mathcal{F}_{I}$, i.e.

$$
r_{l^{2}}=\mathcal{F}_{I}^{T} \mathcal{F}_{I}
$$

The vector $\mathcal{F}_{I}$ represents the discrepancy between the actual measured force $\mathcal{F}_{m}$ and the measurement that a particular set of reaction load parameters $\mathbf{f}_{c}$ would produce. $r_{l^{2}}$ combines violations of each individual constraint equation into a single measure indicating the degree to which the system of constraint equations is violated. The best choice for $\mathbf{f}_{c}$ is the vector which minimizes $r_{l^{2}}$.

Unfortunately, $r_{F I}$ does not have consistent units. To see this we expand equation 6.5 to get

$$
r_{l^{2}}=f_{x_{I}}^{2}+f_{y_{I}}^{2}+m_{V_{O_{I}}}^{2}
$$

where $f_{x_{I}}, f_{y_{l}}$ and $m_{V_{O_{I}}}$ are the componements of $\mathcal{F}_{I}$. The error metric $r_{l^{2}}$ is the sum of two terms which have units of force squared and a third term which has units of 
moment squared. While each individual term in the sum indicates the degree to which its associated constraint equation has been violated, the lack of common dimensions renders the sum of these terms meaningless. If we ignore the units and select a solution vector based on the minimization of equation 6.5 we are effectively assigning an arbitrary relative weighting to the force and moment constraint violations. To avoid these problems we must reformulate the problem in a dimensionally consistent framework which imposes physically justifiable relative weights to the different constraint violation terms. A convenient way to achieve both of these objectives is to instead minimize the violation power, which we define in the following section.

\subsubsection{Violation Power}

The total instantaneous power dissipated by the contact forces as the vehicle moves is

$$
p=\mathcal{F}_{m}^{T} \mathcal{V}_{m}
$$

In section 5.7 we showed that, given a particular candidate contact model and its associated force and velocity constraint equations, we can write each of the measured data vectors $\mathcal{F}_{m}$ and $\mathcal{V}_{m}$ as the sum of a permissible and an impermissible component, i.e.

$$
\begin{aligned}
& \mathcal{F}_{m}=\mathcal{F}_{P}+\mathcal{F}_{I} \\
& \mathcal{V}_{m}=\mathcal{V}_{P}+\mathcal{V}_{I}
\end{aligned}
$$

Using these relations to rewrite 6.7 yields

$$
p=\mathcal{F}_{P}^{T} \mathcal{V}_{P}+\mathcal{F}_{P}^{T} \mathcal{V}_{I}+\mathcal{F}_{I}^{T} \mathcal{V}_{P}+\mathcal{F}_{I}^{T} \mathcal{V}_{I}
$$

The first term is the permissible power dissipation, i.e. the portion of the measured 
power which is consistent with the constraints associated with the presumed contact model. We define the violation power to be the sum of the remaining terms i.e.

$$
p_{v}=\mathcal{F}_{P}^{T} \mathcal{V}_{I}+\mathcal{F}_{I}^{T} \mathcal{V}_{P}+\mathcal{F}_{I}^{T} \mathcal{V}_{I}
$$

The violation power is the portion of the total power dissipation which is physically impossible given the constraints of the candidate contact model under consideration. The violation power will equal zero when both $\mathcal{F}_{I}$ and $\mathcal{V}_{I}$ are zero, i.e. when the candidate contact model is perfectly consistent with the measured data $\mathcal{F}_{m}$ and $\mathcal{V}_{m}$. Because $\mathcal{V}_{I}=\mathbf{0}$ for every candidate contact model we have constructed ( see section $5.5), \mathcal{V}_{P}=\mathcal{V}_{m}$ and equation 6.11 can be further simplified to

$$
p_{v}=\mathcal{F}_{I}^{T} \mathcal{V}_{m}
$$

\subsubsection{Modified Least Squares Solution}

We return now to the problem of solving for the reaction loads at a candidate contact model's presumed contact points. As described above, the violation power is

$$
\begin{aligned}
p_{v} & =\mathcal{F}_{I}^{T} \mathcal{V}_{m}=\mathcal{V}_{m}^{T} \mathcal{F}_{I} \\
& =v_{x} f_{x_{I}}+v_{y} f_{y_{I}}+\omega m_{V_{O_{I}}}
\end{aligned}
$$

Each term in the sum represents the power dissipation associated with the violation of a particular constraint equation. We could choose to select the solution vector $\mathbf{f}_{c}$ which minimizes the square of $p_{v}$ which we can write as

$$
r=\mathcal{F}_{I}^{T} \mathcal{V}_{m} \mathcal{V}_{m}^{T} \mathcal{F}_{I}
$$

Comparing equation 6.15 with equation 6.5 indicates that we have changed the original least squares problem into a weighted least squares pRoblem. While the new $r$ 
is dimensionally consistent, the weighting matrix formed by $\mathcal{V}_{m} \mathcal{V}_{m}^{T}$ is not positive definite. This reflects the fact that the violation power associated with each constraint equation, i.e. the three terms in equation 6.14 , can cancel so $p_{v}$ can equal zero despite the fact that the individual violation power some of the constraint equations are non-zero.

To rectify this problem we rewrite the error metric $r$ such that it can only be zero when all of the individual violation power terms are zero. We can form a vector of these terms $\mathbf{p}_{v}$

$$
\mathbf{p}_{v}=\mathrm{W}_{\mathcal{V}_{m}} \mathcal{F}_{I}
$$

where $\mathbf{W}_{\mathcal{V}_{m}}$ is a diagonal matrix formed from the elements of the vector $\mathcal{V}_{m}$, i.e.

$$
\mathbf{W}_{\nu_{m}}=\left[\begin{array}{ccc}
v_{x} & 0 & 0 \\
0 & v_{y} & 0 \\
0 & 0 & \omega
\end{array}\right]
$$

Using $\mathbf{p}_{v}$ we define $r_{p_{v}}$ to be

$$
\begin{aligned}
r_{p_{v}} & =\mathbf{p}_{v}^{T} \mathbf{p}_{v} \\
& =\mathcal{F}_{I}^{T} \mathbf{W}_{\mathcal{V}_{m}}^{T} \mathbf{W}_{\mathcal{V}_{m}} \mathcal{F}_{I} \\
& =\left(v_{x} f_{x_{I}}\right)^{2}+\left(v_{y} f_{y_{I}}\right)^{2}+\left(\omega m_{V_{O_{I}}}\right)^{2}
\end{aligned}
$$

Comparing equation 6.19 with equation 6.5 indicates that the new version of $r$ again changes the original least squares problem into a weighted least squares problem, but the new weighting matrix $\mathbf{W}_{\mathcal{V}_{m}^{T}}^{T} \mathbf{W}_{\mathcal{V}_{m}}$ is now positive definite as long as the elements of $\mathcal{V}_{m}$ are non-zero. Given that $\mathcal{V}_{m}$ consists of measured values, we only know the element values within the resolution limits of the velocity sensor. Thus we can reasonably replace any zero element on the diagonal of $\mathbf{W}_{\mathcal{V}_{m}}$ by some multiple of the 
appropriate sensor resolution limit to ensure that $W_{\mathcal{V}_{m}}$ is always full rank (Ideally this multiple would be one, but a larger multiple may be required to avoid an ill conditioned $\mathbf{W}_{\mathcal{V}_{m}}$. The actual multiple is not important as long as resulting term is small, i.e. we are substituting a small weight for a zero weight). Thus we can guarantee that $\mathbf{W}_{\mathcal{V}_{m}}$ is always positive definite and, therefore, that $r$ is positive definite as well.

The error metric $r$ is dimensionally consistent and positive definite, i.e. it is only zero when all three force constraint equations for a model are exactly satisfied. Using $\mathcal{F}_{I}=\mathbf{A} \mathbf{f}_{c}$ to eliminate $\mathcal{F}_{I}$ from $r$ we get

$$
r_{p_{v}}=\left(\mathcal{F}_{m}-\mathbf{A} \mathbf{f}_{c}\right)^{T} \mathbf{W}_{\mathcal{V}_{m}}^{T} \mathbf{W}_{\mathcal{V}_{m}}\left(\mathcal{F}_{m}-\mathbf{A} \mathbf{f}_{c}\right)
$$

To find the solution $\mathbf{f}_{c}$ which minimizes $r$ we set the derivative $\frac{d r_{p_{v}}}{d \mathbf{f}_{c}}$ equal to zero and solve for $\mathbf{f}_{c}$.

$$
\begin{aligned}
\frac{d r_{p_{v}}}{d \mathbf{f}_{c}} & =-2 \mathbf{A}^{T} \mathbf{W}_{\mathcal{V}_{m}}^{T} \mathbf{W}_{\mathcal{V}_{m}} \mathcal{F}_{m}+2 \mathbf{A}^{T} \mathbf{W}_{\mathcal{V}_{m}}^{T} \mathbf{W}_{\mathcal{V}_{m}} \mathbf{A f}_{c}=0 \\
\mathbf{f}_{c} & =\left[\mathbf{A}^{T} \mathbf{W}_{\mathcal{V}_{m}}^{T} \mathbf{W}_{\mathcal{V}_{m}} \mathbf{A}\right]^{-1} \mathbf{A}^{T} \mathbf{W}_{\mathcal{V}_{m}}^{T} \mathbf{W}_{\mathcal{V}_{m}} \mathcal{F}_{m}
\end{aligned}
$$

The solution vector $\mathbf{f}_{c}$ is the set of contact reaction load parameters which, given the instantaneous measured data $\mathcal{F}_{m}$ and $\mathcal{V}_{m}$, minimizes the violation of a given candidate contact model's force constraints.

\subsection{Permissible and Impermissible Components of the Measured Force}

In this section we decompose the measured force vector $\mathcal{F}_{m}$ into its permissible and impermissible components $\mathcal{F}_{P}$ and $\mathcal{F}_{I}$ for a given candidate contact model. We then verify that these components are, in fact, always orthogonal. 


\subsubsection{Computing $\mathcal{F}_{P}$}

We can construct a $3 \times 3$ projection matrix $\mathbf{P}_{\mathcal{F}_{P}}$ which directly extracts the permissible component of the measured contact forced $\mathcal{F}_{m}$. The permissible component of $\mathcal{F}_{m}$ is

$$
\begin{aligned}
\mathcal{F}_{P} & =\mathbf{A} \mathbf{f}_{c} \\
& =\mathbf{A}\left[\mathbf{A}^{T} \mathbf{W}_{\mathcal{V}_{m}}^{T} \mathbf{W}_{\mathcal{V}_{m}} \mathbf{A}\right]^{-1} \mathbf{A}^{T} \mathbf{W}_{\mathcal{V}_{m}}^{T} \mathbf{W}_{\mathcal{V}_{m}} \mathcal{F}_{m} \\
& =\mathbf{P}_{\mathcal{F}_{P}} \mathcal{F}_{m}
\end{aligned}
$$

where $\mathbf{P}_{\mathcal{F}_{P}}=\mathbf{A}\left[\mathbf{A}^{T} \mathbf{W}_{\mathcal{V}_{m}}^{T} \mathbf{W}_{\mathcal{\nu}_{m}} \mathbf{A}\right]^{-1} \mathbf{A}^{T} \mathbf{W}_{\mathcal{V}_{m}}^{T} \mathbf{W}_{\mathcal{V}_{m}}$.

\subsubsection{Computing $\mathcal{F}_{I}$}

Similarly, we can construct another $3 \times 3$ matrix to directly extract the impermissible component of $\mathcal{F}_{m}$.

$$
\begin{aligned}
\mathcal{F}_{I} & =\mathcal{F}_{m}-\mathcal{F}_{P} \\
& =\left(\mathbf{I}-\mathbf{P}_{\mathcal{F}_{P}}\right) \mathcal{F}_{m} \\
& =\mathbf{P}_{\mathcal{F}_{I}} \mathcal{F}_{m}
\end{aligned}
$$

\subsubsection{Orthogonality of $\mathcal{F}_{P}$ and $\mathcal{F}_{I}$}

To verify that $\mathcal{F}_{P}$ and $\mathcal{F}_{I}$ are orthogonal we must show that their dot product

$$
\mathcal{F}_{P}^{T} \mathcal{F}_{I}=\mathcal{F}_{m}^{T} \mathbf{P}_{\mathcal{F}_{P}}^{T}\left(\mathbf{I}-\mathbf{P}_{\mathcal{F}_{P}}\right) \mathcal{F}_{m}
$$

is equal to zero for any $\mathcal{F}_{m}$. This will be the case if and only if

$$
\mathbf{P}_{\mathcal{F}_{P}}^{T}\left(\mathbf{I}-\mathbf{P}_{\mathcal{F}_{P}}\right)=0
$$


Note that $\mathbf{P}_{\mathcal{F}_{P}}$ is symmetric, i.e. it is the product of two symmetric matrices $\mathbf{A}\left[\mathbf{A}^{T} \mathbf{W}_{\mathcal{V}_{m}}^{T} \mathbf{W}_{\mathcal{V}_{m}} \mathbf{A}\right]^{-1} \mathbf{A}^{T}$ and $\mathbf{W}_{\mathcal{V}_{m}}^{T} \mathbf{W}_{\mathcal{V}_{m}}$. Substituting $\mathbf{P}_{\mathcal{F}_{P}}^{T}=\mathbf{P}_{\mathcal{F}_{P}}$ into 6.30 and expanding yields

$$
\begin{aligned}
& \mathbf{P}_{\mathcal{F}_{p}}\left(\mathbf{I}-\mathbf{P}_{\mathcal{F}_{P}}\right) \\
& =\left(\mathbf{A}\left[\mathbf{A}^{T} \mathbf{W}_{\mathcal{V}_{m}}^{T} \mathbf{W}_{\mathcal{V}_{m}} \mathbf{A}\right]^{-1} \mathbf{A}^{T} \mathbf{W}_{\mathcal{V}_{m}}^{T} \mathbf{W}_{\mathcal{V}_{m}}\right)\left(\mathbf{I}-\mathbf{A}\left[\mathbf{A}^{T} \mathbf{W}_{\mathcal{V}_{m}}^{T} \mathbf{W}_{\mathcal{V}_{m}} \mathbf{A}\right]^{-1} \mathbf{A}^{T} \mathbf{W}_{\mathcal{V}_{m}}^{T} \mathbf{W}_{\mathcal{V}_{m}}\right) \\
& =\left(\mathbf{A}\left[\mathbf{A}^{T} \mathbf{W}_{\mathcal{V}_{m}}^{T} \mathbf{W}_{\mathcal{V}_{m}} \mathbf{A}\right]^{-1} \mathbf{A}^{T} \mathbf{W}_{\mathcal{V}_{m}}^{T} \mathbf{W}_{\mathcal{V}_{m}}\right)-\left(\mathbf{A}\left[\mathbf{A}^{T} \mathbf{W}_{\mathcal{V}_{m}}^{T} \mathbf{W}_{\mathcal{V}_{m}} \mathbf{A}\right]^{-1} \mathbf{A}^{T} \mathbf{W}_{\mathcal{V}_{m}}^{T} \mathbf{W}_{\mathcal{V}_{m}}\right) \\
& =0
\end{aligned}
$$

Thus $\mathcal{F}_{P}$ and $\mathcal{F}_{I}$ are always orthogonal.

\subsection{Why the Technique Is Limited to Selecting the Best Candidate Model in Each Class}

As noted before, the measured velocity $\mathcal{V}_{m}$ exactly satisfies the velocity constraints of every candidate model we have constructed. In other words, the measured velocity provides no information regarding the relative consistency of the different candidate contact models. While $\mathcal{V}_{m}$ does appear as a weighting term in the consistency measure, the same weights are applied to every model. Thus the consistency measure is a function of the violation of the force constraints alone.

In the solution process we defined a model's impermissible force vector $\mathcal{F}_{I}$ to be the difference between the measured force vector $\mathcal{F}_{m}$ and the best approximation $\mathcal{F}_{P}$ of the measured force vector that could be constructed from vectors lying within the model's permissible force space, i.e. from the columns of the model's A matrix. In general, we expect a model having a permissible force space of dimension two (e.g. any Two Point Contact Model) to produce an inherently smaller impermissible force vector then a model having a permissible force space of dimension one (e.g. any One Point with Slip model). In other words, describing an arbitrary measured 
force vector $\mathcal{F}_{m}$ requires three independent parameters; an approximation involving two independent parameters will always be as good or better than an approximation involving a single parameter. For this reason we can only use the consistency measure to pick the best model within a class, or more accurately, from among models which have permissible force spaces of the same dimension. In the following chapter we present a way to the pick best overall model regardless of the model class.

\subsection{Conclusion}

This chapter presented a technique to determine the best candidate contact model within a model class. We defined two criteria by which we judge candidate models: feasibility and consistency. The manner in which we constructed the candidate models guaranteed their kinematic feasibility. To determine their quasistatic feasibility we solved for the reaction loads required at each model's presumed contact points to produce the measured force/torque vector. To solve for these forces we introduced the concept of violation power, i.e. the power dissipation associated with violation of a model's constraint equation. By properly combining the violation power from each of a model's individual constraint equations we defined a single valued positive definite consistency measure which we used to identify the best feasible model within each model class. Finally, we articulated why the violation power based consistency measure cannot identify the best overall model. 


\section{Chapter 7}

\section{Identifying the Best Overall Model}

In the previous chapter we introduced the concept of the violation power, i.e. the portion of total power dissipation which is physically impossible given the constraints of a particular candidate contact model. We used the violation power to construct a positive definite consistency measure whose value was zero if andonly if the measured data exactly satisfied a model's constraint equations. We used this measure to assess each candidate model's consistency with the instantaneous measured force and velocity, $\mathcal{F}_{m}$ and $\mathcal{V}_{m}$. Our initial expectation was that the model with the smallest measure would be the best candidate model. Instead, we found that this approach only identifies the best model within each class; the violation power based consistency measure cannot determine the best overall candidate contact model.

This result stems from the fact that the instantaneous velocity $\mathcal{V}_{m}$ exactly satisfies the velocity constraint equations of every candidate contact model ( see Section 5.5) With $\mathcal{V}_{I}=\mathbf{0}$ for every model, the violation power based consistency measure became a function of $\mathcal{F}_{I}$ alone. In general, models having two independent reaction loads (e.g. Two Point Contact models) yield inherently smaller $\mathcal{F}_{I}$ than models having a single reaction load (e.g. One Point Contact with Slip models). Therefore the violation power based consistency measure only permits comparison of models which have the same number of independent reaction loads, i.e. models which have permissible force 
spaces of the same dimension.

In this chapter we resolve this problem by considering the vehicle's incremental motion vector $\Delta \mathcal{X}_{m}$, formed by subtracting some previous vehicle position/orientation measurement from the current position/orientation measurement. As discussed in

Section 5.6, $\Delta \boldsymbol{X}_{m}$ ( unlike $\mathcal{V}_{m}$ ) does not inherently satisfy the motion constraints of each candidate contact model. Thus when we decompose $\Delta \mathcal{X}_{m}$ into its permissible and impermissible components, $\Delta \mathcal{X}_{I}$ will in general be nonzero and values of its elements will indicate the degree to which each model's motion constraint equations are violated by the differential motion vector $\Delta \mathcal{X}_{m}$. The decomposition of $\Delta \mathcal{X}_{m}$ is largely analogous to the decomposition of $\mathcal{F}_{m}$ performed in the preceding chapter.

\subsection{Incremental Motion of a Model's Contact Points}

Given an incremental motion of the vehicle, $\Delta \mathcal{X}_{m}$, we seek a solution vector of corresponding, unknown incremental tangential motions $\Delta \mathrm{x}_{c}$ of a model's presumed contact points such that

$$
\Delta \mathcal{X}_{I}=\Delta \mathcal{X}_{m}-\mathrm{B} \Delta \mathrm{x}_{c}
$$

yields $\Delta \mathcal{X}_{I}=\mathbf{0}$.

\subsubsection{Problems with a Direct Least Squares Solution for $\Delta \mathrm{x}_{c}$}

When B is not square (i.e. for One Point Contact and Two Point Contact models) there will, in general, be no solution vector $\Delta \mathrm{x}_{c}$ which $\Delta \mathcal{X}_{I}=0$ for a given incremental motion $\Delta \boldsymbol{X}_{m}$. Normally we would solve a problem of this type by selecting the solution vector $\Delta \mathrm{x}_{c}$ for which minimized the square of the length of the error vector $\Delta \mathcal{X}_{I}$. As was the case with the decomposition of $\mathcal{F}_{m}$, however, the length of $\Delta \mathcal{X}_{I}$ is undefined because the elements of $\Delta \mathcal{X}_{I}$ do not share the same units. The 
square of the length of $\Delta \mathcal{X}_{I}$ is

$$
\Delta \mathcal{X}_{I}^{T} \Delta \mathcal{X}_{I}=\Delta x_{I}^{2}+\Delta y_{I}^{2}+\Delta \theta_{I}^{2}
$$

(where $\Delta x_{I}, \Delta y_{I}$ and $\Delta \theta_{I}$ are the components of $\Delta \mathcal{X}_{I}$ ). i.e. $\Delta \mathcal{X}_{I}^{T} \Delta \mathcal{X}_{I}$ is the sum of two terms which have units of length squared and one term which has units of angular displacement squared. When solving for the reaction loads $\mathbf{f}_{c}$ we employed the concept of the violation power to construct a dimensionally consistent, physically justifiable error metric. To determiine the contact point tangential motions $\Delta \mathrm{x}_{c}$ we use the incremental equivalent of the violation power, the violation energy.

\subsubsection{Violation Energy}

The total work $e$ done by the contact forces during the incremental motion $\Delta \boldsymbol{X}_{m}$ of the vehicle is approximately

$$
e \approx \mathcal{F}_{m}^{T} \Delta \mathcal{X}_{m}
$$

In section 5.6 we showed that given a particular candidate contact model and its associated force and motion constraint equations, we can write each $\mathcal{F}_{m}$ and $\Delta \mathcal{X}_{m}$

as the sum of a permissible and an impermissible component. Doing so, 7.3 becomes

$$
e \approx \mathcal{F}_{P}^{T} \Delta \mathcal{X}_{P}+\mathcal{F}_{P}^{T} \Delta \mathcal{X}_{I}+\mathcal{F}_{I}^{T} \Delta \mathcal{X}_{P}+\mathcal{F}_{I}^{T} \Delta \mathcal{X}_{I}
$$

The first term is the permissible work, i.e. the portion of the total work which is consistent with the constraints associated with the presumed contact model. We define the violation energy to be the sum of the remaining terms i.e.

$$
\begin{aligned}
e_{v} & =\mathcal{F}_{P}^{T} \Delta \mathcal{X}_{I}+\mathcal{F}_{I}^{T} \Delta \mathcal{X}_{P}+\mathcal{F}_{I}^{T} \Delta \mathcal{X}_{I} \\
& =\mathcal{F}_{P}^{T} \Delta \mathcal{X}_{I}+\mathcal{F}_{I}^{T} \Delta \mathcal{X}_{m}
\end{aligned}
$$


The violation energy is the portion of the total work which is physically impossible given the constraints of the candidate contact model under consideration. Like the violation power, the violation energy will equal zero when the candidate contact model is perfectly consistent with the measured data vectors $\mathcal{F}_{m}$ and $\Delta \mathcal{X}_{m}$, i.e. when both $\mathcal{F}_{I}$ and $\Delta \mathcal{X}_{I}$ are zero.

\subsubsection{Modified Least Squares Solution}

Using the concept of the violation energy, we can now construct a dimensionall consistent, physically justifiable error metric which will allow us to solve for the unkown contact point incremental motions $\Delta \mathrm{x}_{c}$. Expanding 7.6 we find that

$$
\begin{aligned}
e_{v}= & f_{x_{P}} \Delta x_{I}+f_{y_{P}} \Delta y_{I}+m_{V_{O_{P}}} \Delta \theta_{I} \\
& +f_{x_{I}} \Delta x_{M}+f_{y_{I}} \Delta y_{M}+m_{V_{O_{I}}} \Delta \theta_{M}
\end{aligned}
$$

As was the case with the violation power, an error metric formed by squaring the violation energy $e_{v}$ directly is only positive semi-definite due to the fact that the individual terms in the sum can cancel. We wish to select a model for which, ideally, each and every individual term in the sum equals zero. Thus our consistency measure should be zero only when this is true. A measure which satisfies this requirement is the sum of the squares of each of the terms in 7.8 , which can be written as

$$
\begin{aligned}
r_{e_{v}} & =\left(\mathbf{W}_{\mathcal{F}_{P}} \Delta \mathcal{X}_{I}\right)^{T}\left(\mathbf{W}_{\mathcal{F}_{P}} \Delta \boldsymbol{X}_{I}\right)+\left(\mathbf{W}_{\mathcal{F}_{I}} \Delta \mathcal{X}_{m}\right)^{T}\left(\mathbf{W}_{\mathcal{F}_{I}} \Delta \mathcal{X}_{m}\right) \\
& =\Delta \mathcal{X}_{I}^{T} \mathbf{W}_{\mathcal{F}_{P}}^{T} \mathbf{W}_{\mathcal{F}_{P}} \Delta \mathcal{X}_{I}+\Delta \mathcal{X}_{m}^{T} \mathbf{W}_{\mathcal{F}_{I}}^{T} \mathbf{W}_{\mathcal{F}_{I}} \Delta \mathcal{X}_{m}
\end{aligned}
$$


where $W_{\mathcal{F}_{P}}$ and $W_{\mathcal{F}_{I}}$ are diagonal weighting matrices formed from the elements of $\mathcal{F}_{P}$ and $\mathcal{F}_{I}$ respectively, i.e.

$$
\begin{aligned}
\mathbf{W}_{\mathcal{F}_{P}} & =\left[\begin{array}{ccc}
f_{x_{P}} & 0 & 0 \\
0 & f_{y_{P}} & 0 \\
0 & 0 & m_{V_{O_{P}}}
\end{array}\right] \\
\mathbf{W}_{\mathcal{F}_{I}} & =\left[\begin{array}{ccc}
f_{x_{I}} & 0 & 0 \\
0 & f_{y_{I}} & 0 \\
0 & 0 & m_{V_{O_{I}}}
\end{array}\right] .
\end{aligned}
$$

$\mathcal{F}_{P}$ and $\mathcal{F}_{I}$ are the permissible and impermissible components of $\mathcal{F}_{m}$ we obtained in the previous chapter using the violation power based consistency measure, i.e. we obtain $\mathcal{F}_{P}$ and $\mathcal{F}_{I}$ from equations 6.25 and 6.28. By using these values we are assuming that the reaction loads at the contact points remain essentially constant during the course of the incremental motion $\Delta \mathcal{X}_{m}$. For small incremental motions, this assumption should be reasonable. Using arguments similar to those presented in Section 6.2.3, we can guarantee the positive definiteness of matrices $\mathbf{W}_{\mathcal{F}_{P}}^{T} \mathbf{W}_{\mathcal{F}_{P}}$ and $\mathbf{W}_{\mathcal{F}_{I}}^{T} \mathbf{W}_{\mathcal{F}_{I}}$; therefore $r_{e_{v}}$ is a positive definite error metric.

Using $\Delta \mathcal{X}_{I}=\Delta \mathcal{X}_{m}-\mathrm{B} \Delta \mathrm{x}_{c}$ to eliminate $\Delta \mathcal{X}_{I}$ from $r$ yields

$$
\begin{aligned}
& r_{e_{v}}=\left(\Delta \mathcal{X}_{m}-\mathrm{B} \Delta \mathbf{x}_{c}\right)^{T} \mathbf{W}_{\mathcal{F}_{P}}^{T} \mathbf{W}_{\mathcal{F}_{P}}\left(\Delta \mathcal{X}_{m}-\mathrm{B} \Delta \mathbf{x}_{c}\right) \\
&+\Delta \mathcal{X}_{m}^{T} \mathbf{W}_{\mathcal{F}_{I}}^{T} \mathbf{W}_{\mathcal{F}_{I}} \Delta \mathcal{X}_{m}
\end{aligned}
$$

By using the the violation energy based metric we have tranformed the original least squares problem into a weighted least squares problem. To find the solution $\Delta \mathbf{x}_{c}$ which minimizes $r$ we set the derivative $\frac{d r_{e_{v}}}{d \Delta \mathrm{x}_{c}}$ equal to zero and solve for $\Delta \mathrm{x}_{c}$.

$$
\begin{aligned}
\frac{d r_{e_{v}}}{d \mathbf{f}_{c}} & =0 \\
& =-2 \mathbf{B}^{T} \mathbf{W}_{\mathcal{F}_{P}}^{T} \mathbf{W}_{\mathcal{F}_{P}} \Delta \mathcal{X}_{m}+2 \mathbf{B}^{T} \mathbf{W}_{\mathcal{F}_{P}}^{T} \mathbf{W}_{\mathcal{F}_{P}} \mathbf{B} \Delta \mathbf{x}_{c}
\end{aligned}
$$




$$
\Delta \mathbf{x}_{c}=\left[\mathbf{B}^{T} \mathbf{W}_{\mathcal{F}_{P}}^{T} \mathbf{W}_{\mathcal{F}_{P}} \mathbf{B}\right]^{-1} \mathbf{B}^{T} \mathbf{W}_{\mathcal{F}_{P}}^{T} \mathbf{W}_{\mathcal{F}_{P}}^{T} \mathbf{W}_{\mathcal{F}_{P}} \Delta \boldsymbol{X}_{m}
$$

The solution vector $\Delta \mathrm{x}_{c}$ is the set of contact point motions which, given the current vehicle incremental motion vector $\Delta \mathcal{X}_{m}$, minimizes the violation of a given candidate contact model's incremental motion constraints.

\subsection{Permissible and Impermissible Components of the Incremental Motion Vector}

In this section we decompose the incremental motion vector $\Delta \mathcal{X}_{m}$ into its permissible and impermissible components $\Delta \mathcal{X}_{P}$ and $\Delta \mathcal{X}_{I}$ for a given candidate contact model. This process is analagous to that used to decompose the measured force vector $\mathcal{F}_{m}$.

\subsubsection{Computing $\Delta \mathcal{X}_{P}$}

We can construct a $3 \times 3$ projection matrix $\mathbf{P}_{\mathcal{X}_{P}}$ which directly extracts the permissible component of the measured contact forced $\Delta \mathcal{X}_{m}$. The permissible component of $\Delta \mathcal{X}_{m}$ is

$$
\begin{aligned}
\Delta \mathcal{X}_{P} & =\mathbf{B} \Delta \mathbf{x}_{c} \\
& =\mathbf{B}\left[\mathbf{B}^{T} \mathbf{W}_{\mathcal{F}_{P}}^{T} \mathbf{W}_{\mathcal{F}_{P}} \mathbf{B}\right]^{-1} \mathbf{B}^{T} \mathbf{W}_{\mathcal{F}_{P}}^{T} \mathbf{W}_{\mathcal{F}_{P}} \Delta \mathcal{X}_{m} \\
& =\mathbf{P}_{\mathcal{X}_{P}} \Delta \mathcal{X}_{m}
\end{aligned}
$$

where $\mathbf{P}_{\mathcal{X}_{P}}=\mathbf{B}\left[\mathbf{B}^{T} \mathbf{W}_{\mathcal{F}_{P}}^{T} \mathbf{W}_{\mathcal{F}_{P}} \mathbf{B}\right]^{-1} \mathbf{B}^{T} \mathbf{W}_{\mathcal{F}_{P}}^{T} \mathbf{W}_{\mathcal{F}_{P}}$ 


\subsubsection{Computing $\Delta \boldsymbol{X}_{I}$}

Similarly, we can construct another $3 \times 3$ matrix $\mathbf{P}_{\mathcal{X}_{I}}$ to directly extract the impermissible component of $\Delta \mathcal{X}_{m}$,

$$
\begin{aligned}
\Delta \mathcal{X}_{I} & =\Delta \mathcal{X}_{m}-\Delta \mathcal{X}_{P} \\
& =\left(\mathrm{I}-\mathrm{P}_{\mathcal{X}_{P}}\right) \Delta \mathcal{X}_{m} \\
& =\mathbf{P}_{\mathcal{X}_{I}} \Delta \mathcal{X}_{m}
\end{aligned}
$$

where $\mathbf{P}_{\mathcal{X}_{I}}=\mathbf{I}-\mathbf{P}_{\mathcal{X}_{P}^{\prime}}$.

\subsection{Conclusion}

This chapter introduced the concept of violation energy and used it to define a single valued positive definite consistency measure which we used to identify which of the best-of-class models from Chapter 6 was the best overall model. 


\section{Chapter 8}

\section{Experimental Verification}

\subsection{Introduction}

In this chapter we test the contact identification scheme developed in the preceding chapters. We show that the violation power based metric $r_{p_{v}}$ is a good indicator of the best model within a model class. Using the same trials, we show that the violation energy based metric $r_{e_{v}}$ performs well in identifying which of the best-of-class models identified using $r_{p_{v}}$ is the best overall model. We investigate the sensitivity of the technique to errors in the assumed value of the coefficient of friction, finding that poor estimates of $\mu_{d}$ lead to poor identification results. We conclude with an experimental investigation of the uniqueness of apparently equivalent models, showing that the models are in fact distinct.

\subsection{Experimental Apparatus}

The experiments presented in this chapter, like those presented in Chapter 4, were performed using the the Air Table Vehicle Simulator (ATVS) system shown in Figure 8-1 (see Appendix A for a detailed description of the system design and its characteristics). Briefly, the system consists of a vehicle which, supported by three air bearings, 


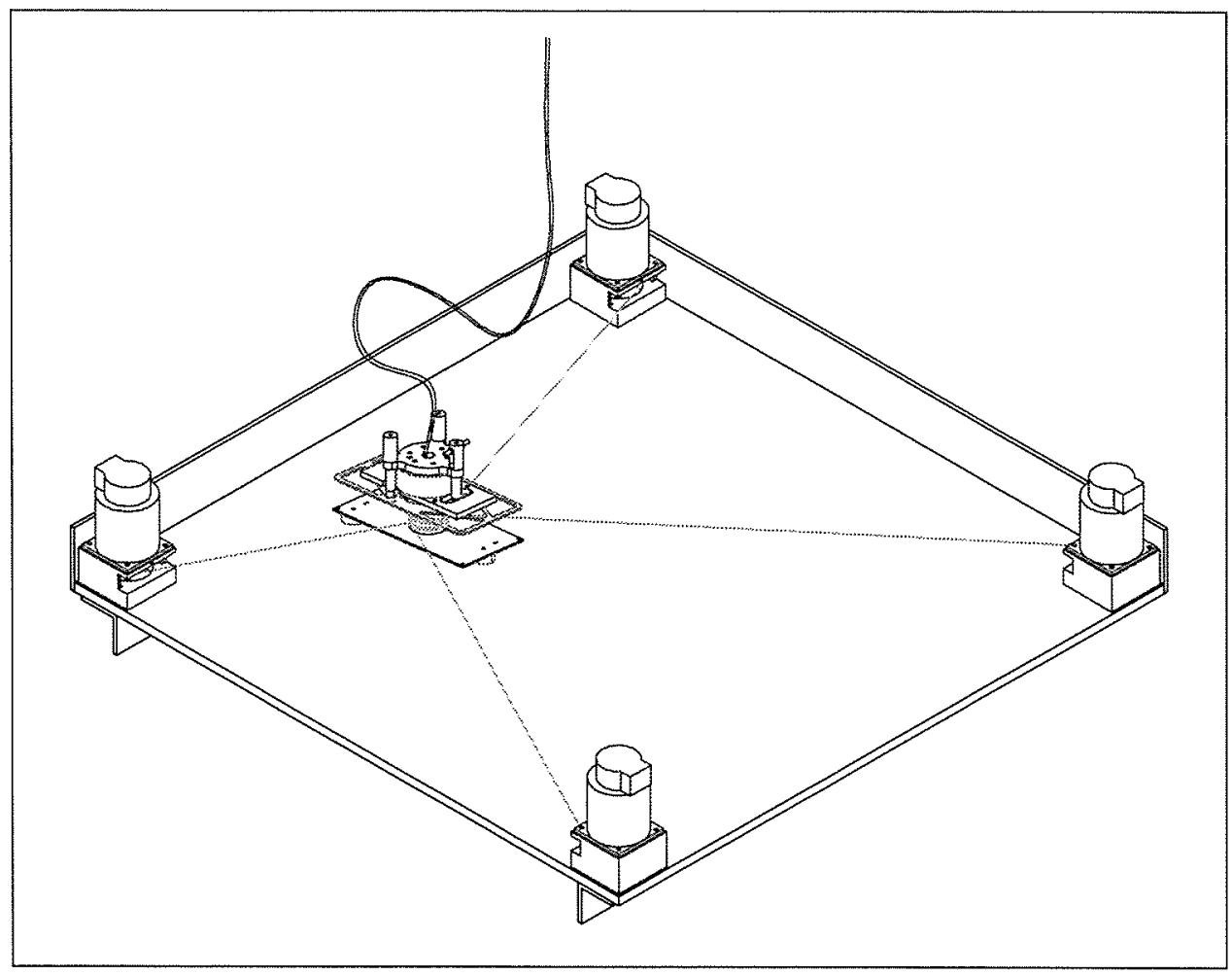

Figure 8-1: The Air Table Vehicle Simulator (ATVS) system consists of a 0.145 by 0.290 meter, air bearing supported vehicle which moves freely over the surface of a one square meter glass topped table surface. Four miniature steel cables couple the motion of the vehicle to that of the four motors mounted to the corners of the table. In general, the position and orientation of the vehicle is controlled by coordinating the motion of the four motors. The position and orientation, as well as the linear and angular velocities, of the vehicle are determined from the motor shaft positions and angular velocities as measured by optical encoders mounted to the motor shafts. For all the experiments performed in this thesis, however, the vehicle was moved by hand i.e. the actuators were only used to maintain tension in the cables. This system is described in greater detail in Appendix A 
moves freely over the surface of a one meter square glass topped table. Four miniature steel cables couple the motion of the vehicle to that of four brushless D.C. servomotors mounted to the table corners. Optical encoders measure the rotation angle and velocity of each motor, enabling the determination of the vehicle position/orientation and velocity with respect to the table. For the experiments in this chapter the vehicle was moved by hand, i.e. the motors were only used to maintain nominal tensions in the cables. The vehicle boundary is identical to that shown in Figure 4-2 A.

For these tests, no filtering was performed on the vehicle velocity data, i.e. the ICR location and the znv point locations were computed directly from the raw vehicle world frame velocity vector. The vehicle world frame position and velocity vectors were computed from the motor shaft angles velocities as described in Appendix A

\subsection{Comments on Measuring the Accuracy of the Identification Technique}

Determining the accuracy of the technique requires that we know the actual contact points during each trial. In each trial the vehicle was moved by hand and brought into contact with fixed environmental objects having known location and geometry. The nominal contact points for each trial were determined visually by plotting the vehicle and obstacle geometries at each time point in a trial and noting apparent points of contact. This determination also considered the measured contact force at each time step to determine at what times contact transitions occurred. We treat these nominal contact points as the actual contact points for each trial. While not perfectly accurate, discrepancies should be small and thus the estimated accuracies should give a good indication of the technique's efficacy. 


\subsection{Using the Violation Power Based Consistency Measure $r_{p_{v}}$ to Select the Best Model in a Class}

In Chapter 6 we showed that the violation power based consistency measure $r_{p_{v}}$ can be used to identify the best candidate contact model within a model class. In this section we test this contention by examining data from four experimental trials for which we know the actual contact model.

\subsubsection{Case 1: One Point Contact with No Slip (Fixed Point Rotation)}

In this experiment, edge 5 of the vehicle was brought into contact with the corner of a rectangular aluminum bar and the vehicle was rotated about the corner such that little or no slip occurred at the contact point (see Figure 8-2).

The upper portion of Figure 8-3 shows $r_{p_{v}}$ for each of the eight possible One Point contact models (i.e. one for each segment in the vehicle boundary) while the lower portion shows which of these models yields the smallest value of $r_{p_{v}}$ at each time point. Clearly, for this trial, $r_{p_{v}}$ is an excellent indicator of the correct model within the One Point model class ( note that Figure 8-3 is a semilog plot; the $r_{p_{v}}$ value for model 5 is typcally an orderof magnitude or more smaller than the next best model). The vehicle is initially at rest at $t=0$ and the velocity goes through zero again at about $t=2.15$ seconds. Very near these times the magnitude of the vehicle velocity is too small to yield reliable readings from the velocity sensors and the technique selects the incorrect model. At all other times, however, the minimum $r_{p_{v}}$ model identifies the correct model.

General note: When a candidate contact model is determined to be infeasible, we set its $r_{p_{v}}$ and $r_{e_{v}}$ values to their worst possible values, i.e. we compute the measures assuming that $\mathcal{F}_{I}=\mathcal{F}_{m}, \mathcal{V}_{I}=\mathcal{V}_{m}$, and $\Delta \mathcal{X}_{I}=\Delta \mathcal{X}_{m}$. This explains why the eight One Point Contact model curves plotted in the upper portion of Figure 8-3 appear 


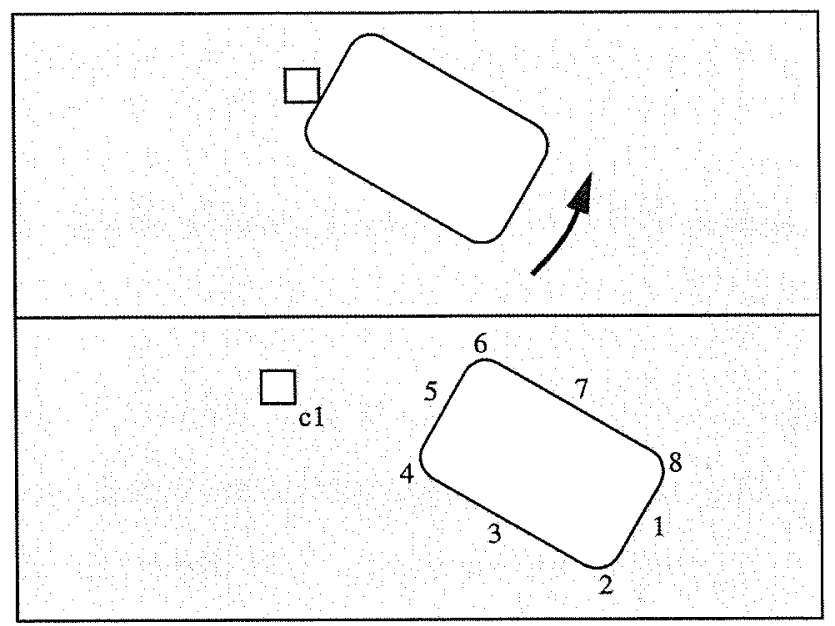

Figure 8-2: Case 1 Experiment: Edge 5 of the vehicle was brought into contact with corner $\mathrm{c} 1$ of a fixed object in environment. The vehicle was then rotated about this corner such that little or no slip occurred at the contact point. Initial rotation was the counter clockwise direction, then the rotation was reversed, bringing the vehicle (approximately) back to its original position. The obstacle was a square bar of 6061 aluminum alloy.
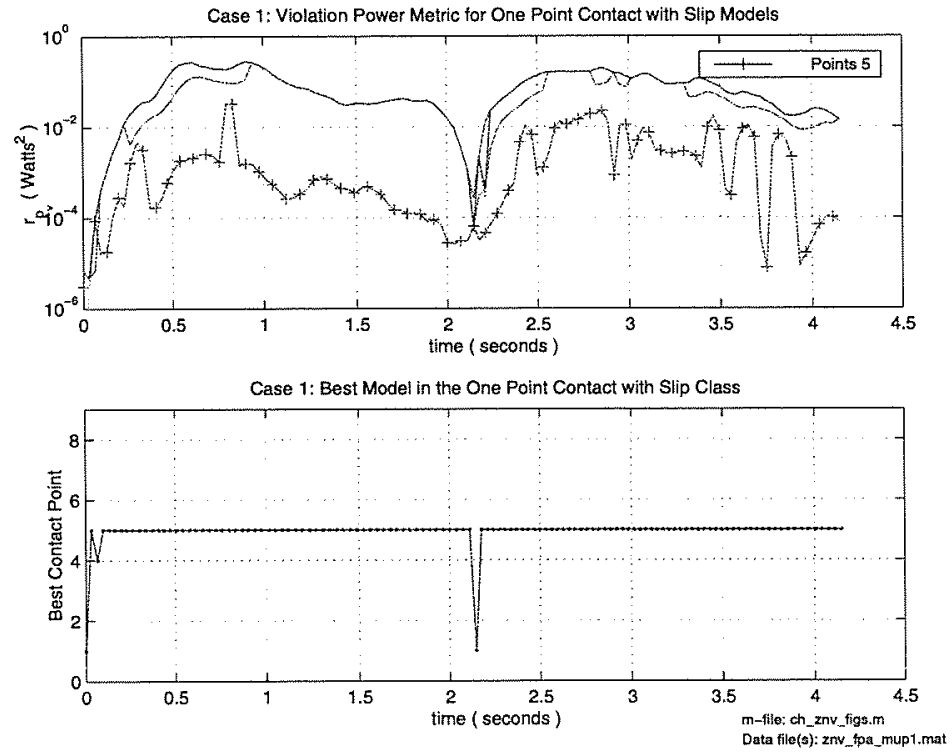

Figure 8-3: Case 1: Violation power based consistency measure $r_{p_{v}}$ for the eight One Point Contact models (upper plot). Best (i.e. minimum $r_{p_{v}}$ ) One Point Contact Model(lower plot). 
to be only three curves; most of the one point models are infeasible for this trial so they overlay each other, forming the uppermost curve in the plot.

\subsubsection{Case 2: One Point Contact with Slip (Rotation and Translation)}

In this experiment vehicle edge 7 was brought into contact with a fixed cylindrical post (see Figure 8-4). The vehicle was then moved by hand such that both slip at and rotation about the contact point occurred. The direction of slip was such that the actual contact point approached arc 6 of the vehicle outline.

The upper plot in Figure 8-5 shows the violation power for each of the eight possible one point contact models for the data set while the lower plot shows the number of the model having the smallest violation power at each point in time. We see that the One Point Contact with Slip model associated with edge 7 has the lowest violation power up until about time $t=1.75$ seconds. Note that as the trial progresses the violation power associated with model 6 grows continually smaller, reflecting the fact that the actual contact point gets closer and closer to arc 6 throughout the trial. Eventually the violation power for model 6 becomes smaller than that for model 7 , at which point model 6 is selected as the most likely model, as indicated in the lower of the two plots in Figure 8-5.

During the trial, the actual contact point never quite reaches arc 6, i.e. the selection technique prematurely identifies arc 6 as the contacted boundary segment. If the actual contact point reached the intersection between edge 7 and arc 6 we would expect both models to be equivalent, i.e. both models would have the same contact point, the same normal and tangential vectors and therefore the same permissible force and velocity spaces; they would be the same model. Thus when the contact point is in the vicinity of the intersection point, we expect both models to be very similar and therefore to be difficult to distinguish from one another. Given that the measurements are not perfect and that the assumed coefficient of friction $\left(\mu_{d}=.25\right.$ for this trial) is 


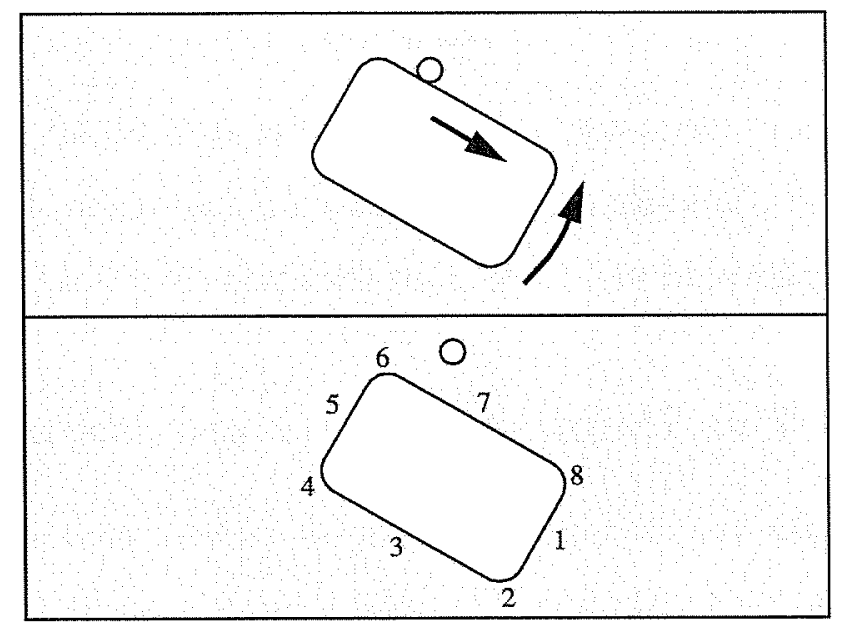

Figure 8-4: Case 2 Experiment: Edge 5 of the vehicle was brought into contact with a fixed cylindrical obstacle. The vehicle was then moved such that it was in sliding contact, where the vehicle motion combined both rotation and translation of the vehicle relative to the contact point. The obstacle was a $12.7 \mathrm{~mm}$ diameter stainless steel bar.
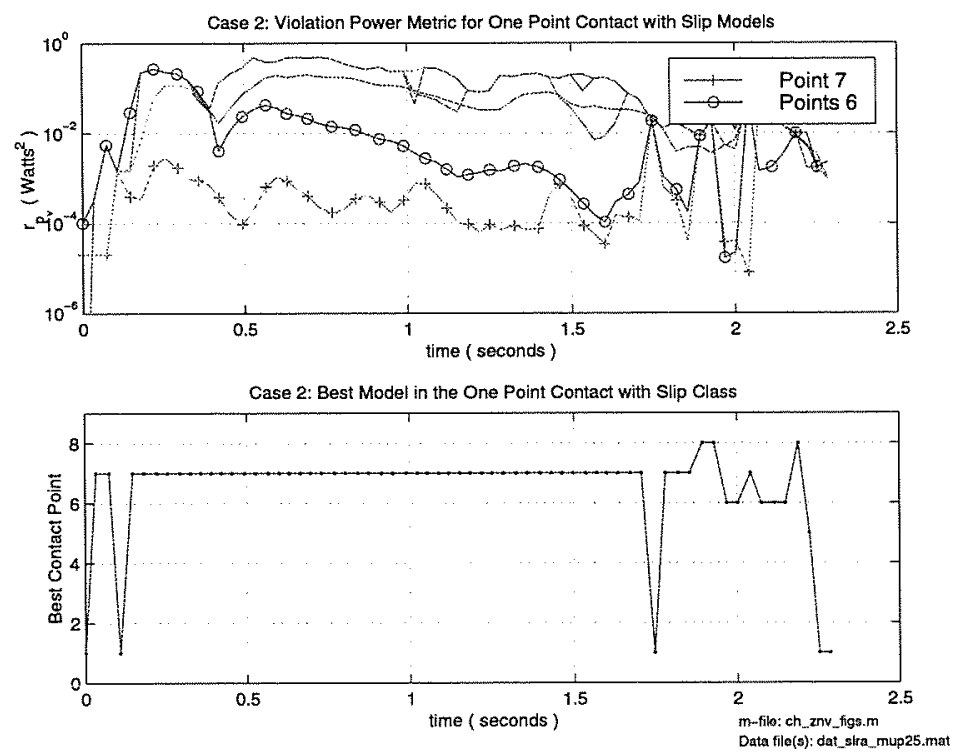

Figure 8-5: Case 2: Violation power based consistency measure $r_{p_{v}}$ for the eight One Point Contact models (upper plot). Best (i.e. minimum $r_{p_{v}}$ ) One Point Contact Model(lower plot). 
not exactly correct, the computed violation power for model 6 prematurely transitions to a value lower than that for model 7 . Higher resolution measured data and better estimates of the actual coefficient of friction would lead to better results. Given that the properties of the two models are very similar in this region, however, we expect that using either model would yield adequate results in practice.

\subsubsection{Case 3: Two Point Contact (Pure Translation)}

In this trial edge 5 of the vehicle was brought into contact with a flat aluminum plate and the vehicle moved by hand such that vehicle underwent pure translation along the face of the plate (see Figure 8-6). The vehicle was initially moved in the positive (vehicle frame) y-direction, was brought to a stop at approximately time $t=2.6$ seconds, and was then moved in the negative y-direction, back towards its starting position.

The upper portion of Figure 8-7 shows the violation power for the Two Point Contact models for this trial while the lower portion shows the two point model having the smallest violation power. The y-axis on the lower plot indicates the combination of boundary segments associated with each of the Two Point Contact models. For example, in the first half of the trial, the best model is the $(5,6)$, i.e. the model in which contact occurs on edge 5 and arc 6 . In the vicinity of the velocity reversal ( $t \approx 2.6$ seconds) the magnitude of the vehicle velocity is too small to yield reliable readings from the velocity sensors and the technique selects an incorrect model. Once the reversed velocity grows large enough, model $(4,6)$ is identified as the best Two Point Contact model for most of the remainder of the trial. Note from the upper plot, however, that model $(4,6)$ is nearly indistinguishable from model $(5,6)$ in the first half of the trial and is nearly indistinguishable from model $(4,5)$ in the latter half of the trial. This reflects the fact that, in these regions, the appropriate pair of models are equivalent. 


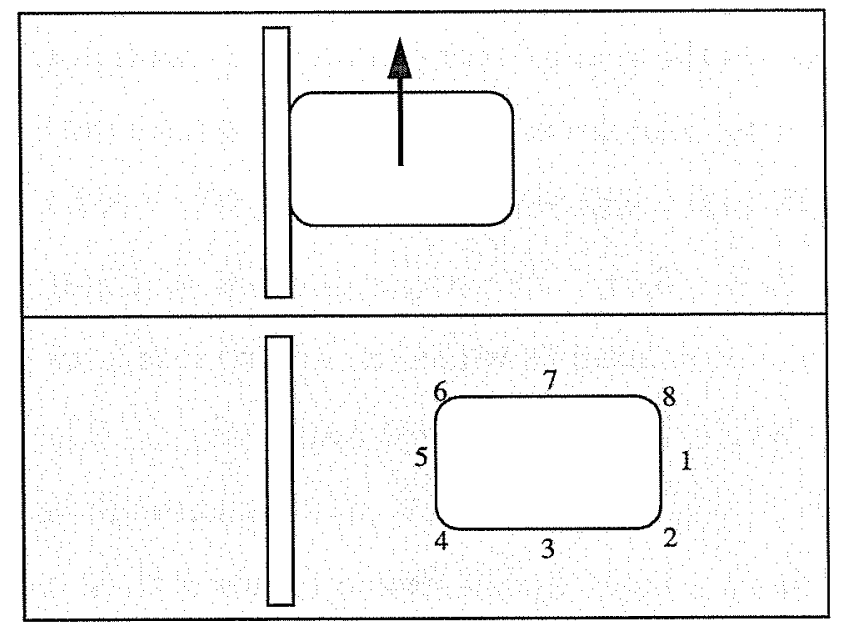

Figure 8-6: Case 3 Pure Translation: Edge 5 of the vehicle was brought into full contact with a flat wall.. This contact was maintained throughout the trial as the vehicle was translated first upward and then downward back to its approximate initial position. The wall was smooth, rectangular block of 6061 aluminum.
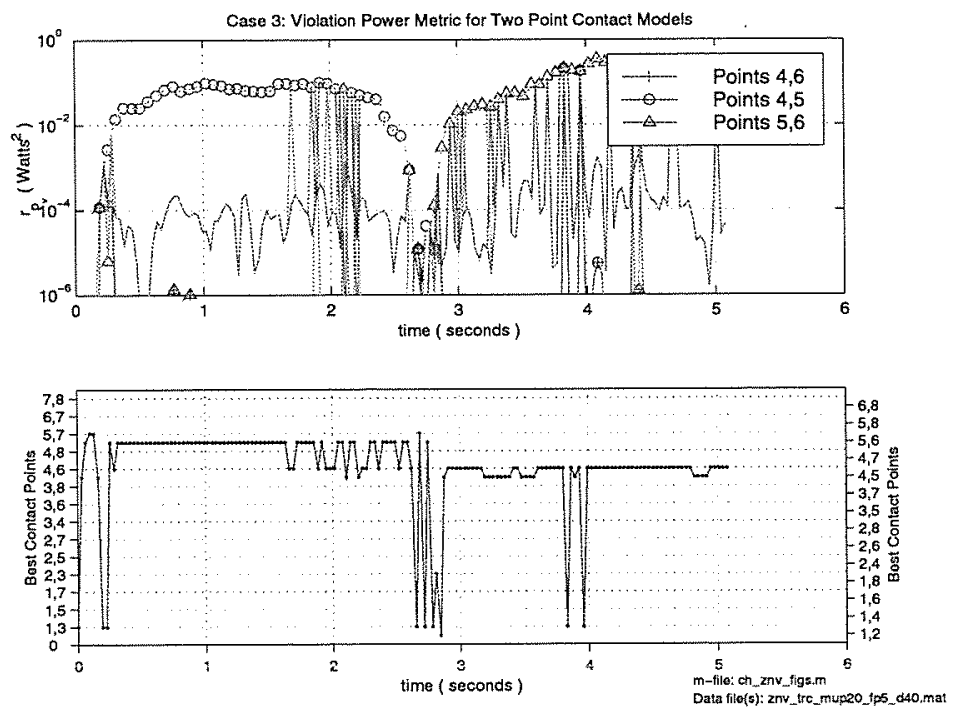

Figure 8-7: Case 3: Violation power based consistency measure $r_{p_{v}}$ for the twentyeight Two Point Contact models (upper plot). Best (i.e. minimum $r_{p_{v}}$ ) Two Point Contact Model(lower plot). 


\subsubsection{Case 4: Two Point Contact ( Rotation and Translation)}

In this trial edges 3 and 5 of the vehicle were brought into contact with fixed cylindrical posts(see Figure 8-8). The vehicle was then rotated in the clockwise direction such that contact with both posts was maintained, with the actual contact eventually transitioning from $(3,5)$ to $(3,4)$. The upper portion of Figure 8-9 shows $r_{p_{v}}$ for each of the possible two point models while the lower portion of the figure plots the number of the model which has the smallest value of $r_{p_{v}}$. The upper plot clearly identifies models $(3,4)$ and $(3,5)$ as the best models throughout the trial, but the similarity between their $r_{p_{v}}$ values makes choice between these two models difficult. The lower plot, based on a direct comparison of the $r_{p_{v}}$ values, reflects this difficulty in the numerous transitions between the two models. The similarity of the $r_{p_{v}}$ values raises the question of whether these two models are fundamentally equivalent not. We consider this issue in greater detail in Section 8.8.

\subsection{Using the Violation Energy to Select the Best Best-of-Class Model}

In the previous section we showed that consistency measure $r_{p_{v}}$ does a good job of identifying the actual contact model if we already know the correct model class. In general we do not know the model class, so we can only use $r_{p_{v}}$ to identify the best model within each model class. In Chapter 7 we presented the violation energy based consistency measure $r_{e_{v}}$ as a means by which to determine which of the best-of-class models is the best overall model. In this section we test this ability of this technique to select the best overall model from the best-of-class models associated with each of the data sets discussed in the previous section. 


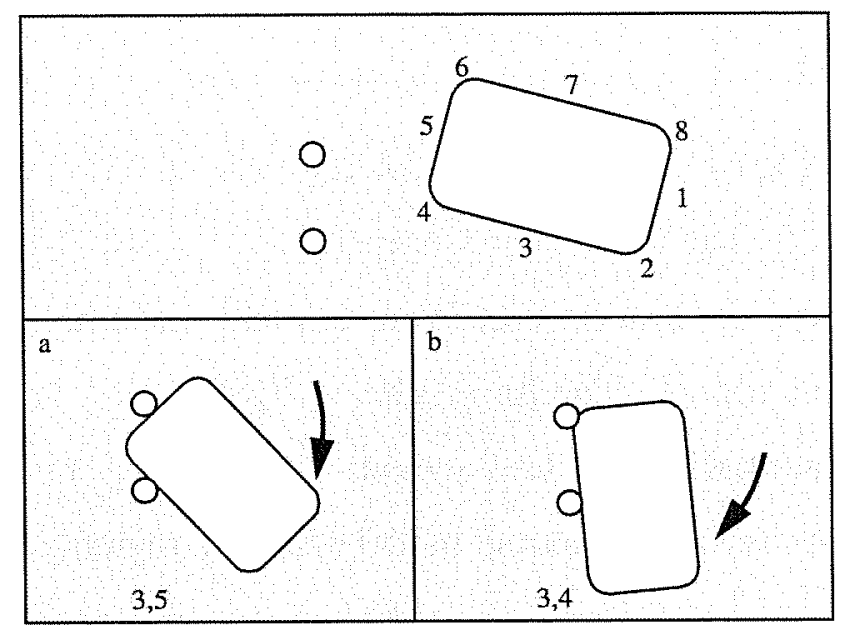

Figure 8-8: Case 4 Experiment: The vehicle was brought into contact with two fixed cylindrical obstacles and then rotated in a clockwise direction. Initially vehicle edges 3 and 5 contacted the cylinders( we shall refer to this contact configuration as simply $(3,5))$. As the vehicle rotates, the actual contact state eventually transitions from from $(3,5)$ to $(3,4)$. Both obstacles were $12.7 \mathrm{~mm}$ diameter stainless steel bars.
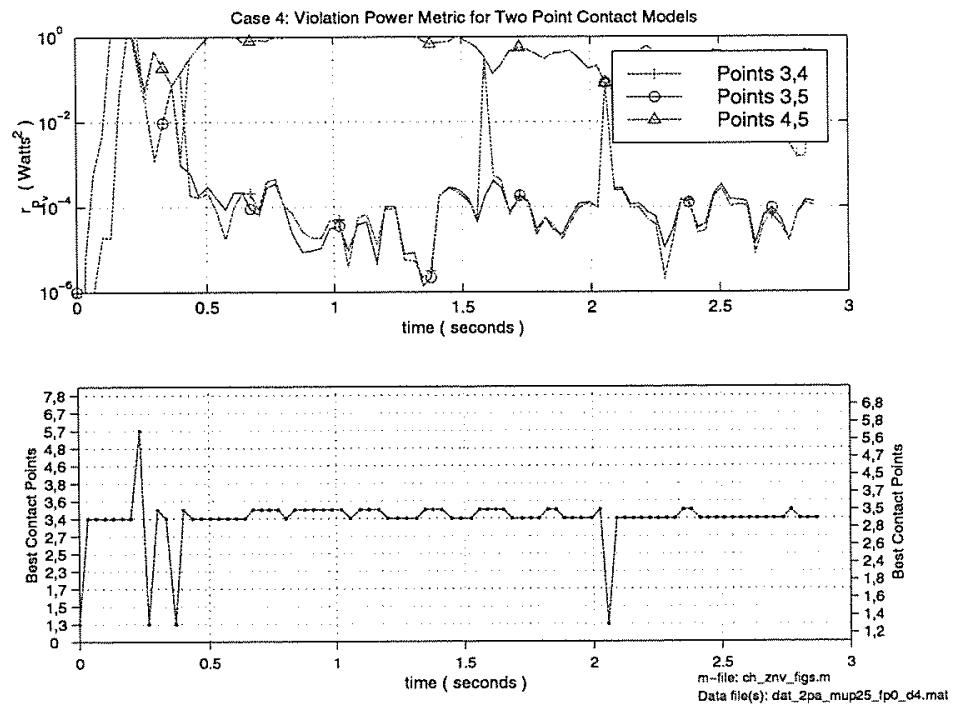

Figure 8-9: Case 4: Violation power based consistency measure $r_{p_{v}}$ for the twentyeight Two Point Contact models (upper plot). Best (i.e. minimum $r_{p_{v}}$ ) Two Point Contact Model(lower plot). 


\subsubsection{Case 1: One Point Contact with No Slip (Fixed Point Rotation)}

The upper portion of Figure 8-10 shows $r_{e_{v}}$ for the best One Point Contact and Two Point Contact models for the One Point Contact with No Slip data set. We see that the consistency measure for the best One Point Model is uniformly smaller than that for the best Two Point Model. The lower plot in Figure 8-10 shows the number of contact points associated with the best overall model (dots) in comparison to the number of contact points in the actual model (shaded region). For this trial the technique always selects a model with the right number of contact points.

Figure 8-11 shows the specific model selected as a function of time. The y-axis shows all of the possible one and two point contact scenarios for the given vehicle shape. The eight One Point Contact scenarios are shown at the bottom where the numbers 1 through 8 signify the number of the vehicle boundary segment in contact with the environment. The twenty-eight possible two point contact scenarios are shown above the one point models. Each pairing of numbers $(i, j)$ indicates a particular two point contact scenario in which boundary segments $i$ and $j$ are in contact with the environment. The plot shows the best overall model selected for each time points (dots) in comparison to the actual contact scenario (shaded region). For this trial the technique is seen to select the correct model almost every time.

\subsubsection{Case 2: One Point Contact with Slip (Rotation and Translation)}

The upper portion of Figure 8-12 shows $r_{e_{v}}$ for the best of the One Point Contact model and for the bestTwo Point Contact model while the lower portion indicates which of these best-of-class models has the smallest value of $r_{e_{v}}$. We see that selecting the best-of-class model with the minimum $r_{e_{v}}$ model typically identifies the correct number of contact points in the actual model but that there are regions where it 

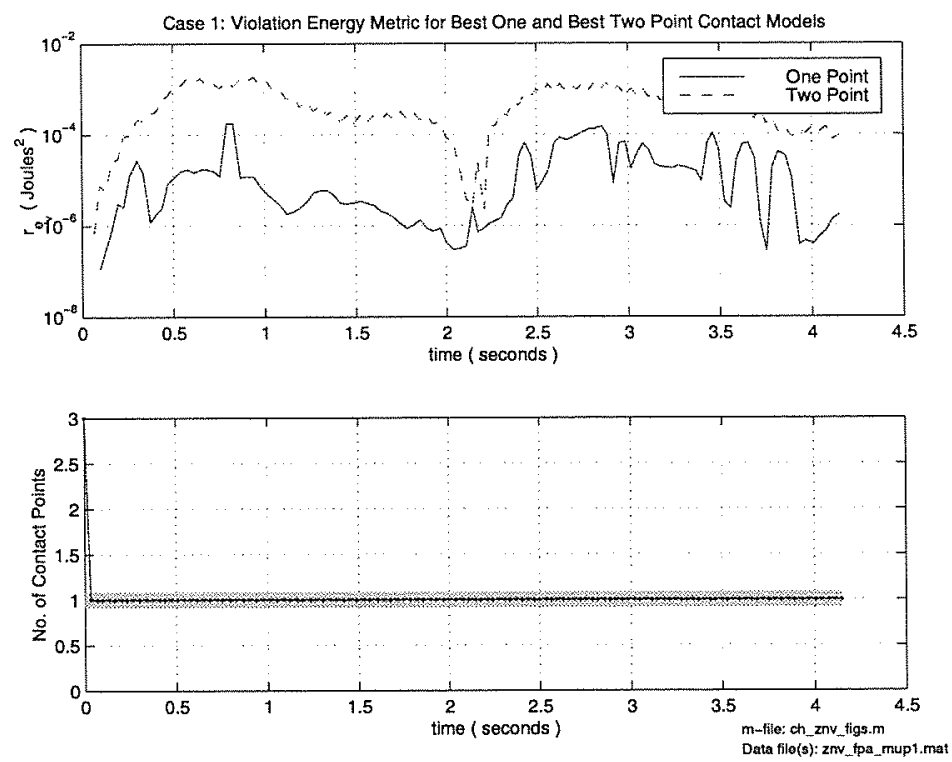

Figure 8-10: Case 1: Violation energy measure $r_{e_{v}}$ the best One Point Contact model and the best Two Point Contact model (upper plot). Number of contact points associated with the best overall model (lower plot).

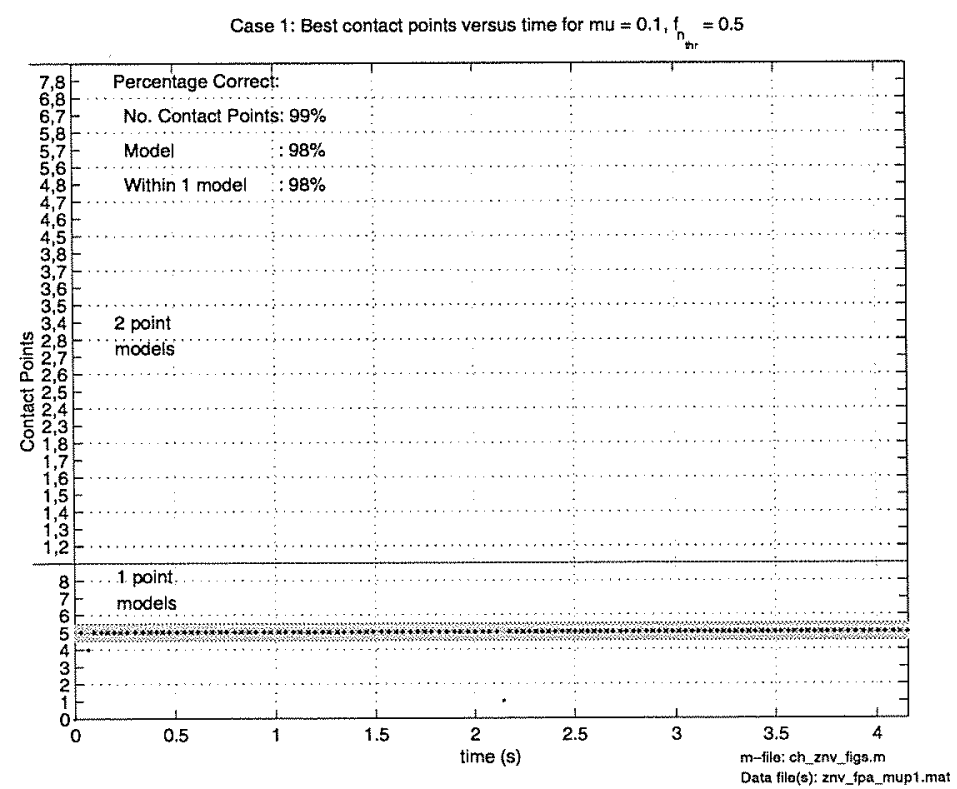

Figure 8-11: Case 1: Best Overall Model vs. Time. 
incorrectly selects a two point model over the correct one point model. Figure 813 shows the actual model selected as a function of time. The approach typically identifies the correct contact model (i.e. One Point Contact on edge 7 of the vehicle).

\subsubsection{Case 3: Two Point Contact ( Pure Translation)}

In this trial the vehicle's motion (theoretically) exactly satisfies the motion constraint equations of all the One Point and Two Point contact models associated with edge 5 (i.e. One Point models (4), (5), (6) and Two Point models $(4,5),(4,6)$ and $(5,6))$. Under these conditions the ability to choose between models becomes entirely a function of how well the various models' force constraints are satisfied. Therefore, using arguments analogous to those presented in section 6.4, we expect the violation energy based technique to select, in general, Two Point contact models for the pure translation case.

Referring to the lower portion of Figure 8-14, we do not see the expected preference for Two Point models, i.e. in the first half of the trial, the technique typically selects a One Point model. Referring to Figure 8-15, however, we see that the One Point model selected is almost always one of the models associated with edge 5 (i.e. models (4), (5) and (6) ). During the second half of the trial, the technique typically selects Two Point models and Figure 8-15 shows that the Two Point models selected are always one of the three associated with edge 5 (i.e. models $(4,5),(4,6)$ and $(5,6)$ ). Thus the technique, with few exceptions, selects reasonable models. The question remains as to why the expected preference for Two Point models is absent from the first half of the trial. The answer is twofold.

Figure 8-16 shows the estimated coefficient of friction $\mu_{d}$ for the trial. To compute this value we divided the measured force's tangential component by its normal component and took the absolute value ( the near zero values at times $t=0$ and $t \approx 2.7$

correspond to times when the tangential component was zero, i.e. before the vehicle was moved and during reversal of the vehicle velocity, respectively). The value of $\mu_{d}$ 

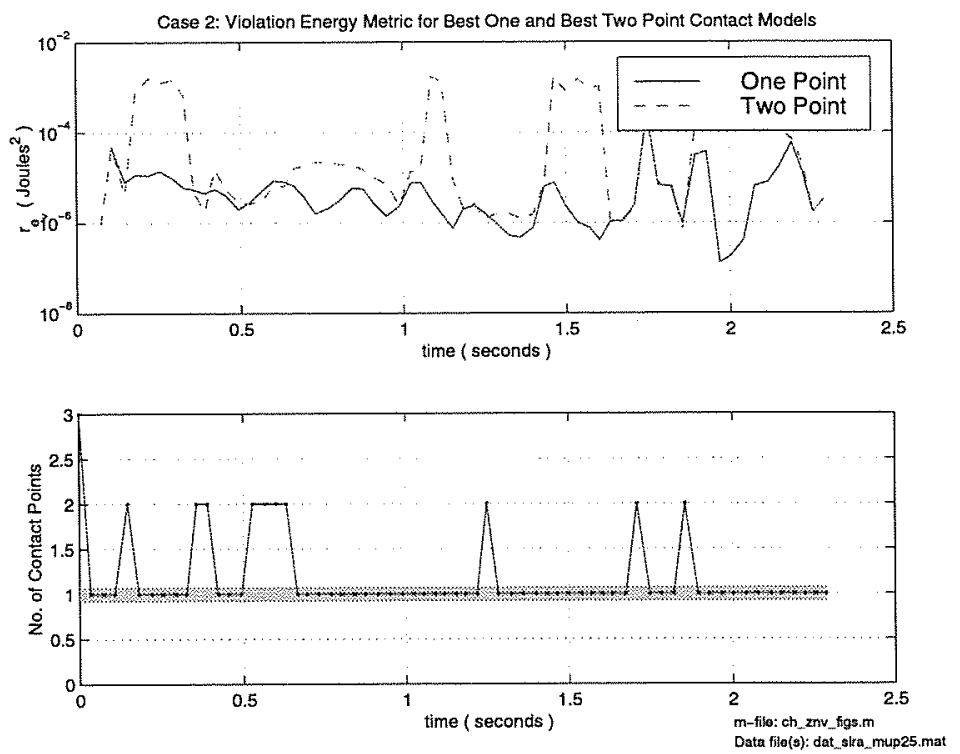

Figure 8-12: Case 2: Violation energy measure $r_{e_{v}}$ the best One Point Contact model and the best Two Point Contact model (upper plot). Number of contact points associated with the best overall model. (lower plot).

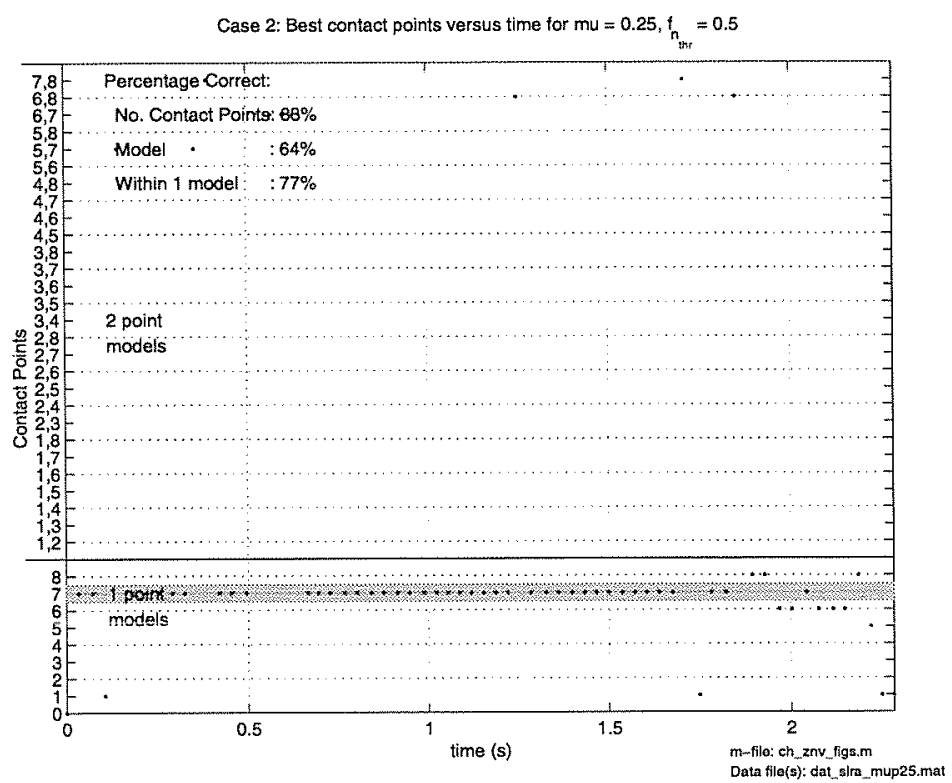

Figure 8-13: Case 2: Best Overall Model vs. Time. 
used by the technique for Case 3 was $\mu_{d}=.2$. Thus we see that the assumed value for $\mu_{d}$ was too high in the first half of the trial and was too low in the latter half. We believe this variation in the actual coefficient of friction is partly responsible for the absence of the expected preference for Two Point models for Case 3.

A second contributing factor is the kinematics of the ATVS mechanism. We do not directly measure the vehicle's position and velocity; we compute them from measurements of the motor angles and velocities. Small errors in our knowledge of the pulley and cable diameters and the discrete nature of the encoder measurements lead to a computed vehicle trajectory which is not pure translation. Thus the "theoretically" exact satisfaction of the models' motion constraints does not occur, i.e. the computed vehicle trajectory includes some small amount of rotation. Rotation of the vehicle is permissible for One Point models but not for Two Point models. Thus, what we thought would be a bias towards Two Point models could actually be, if the kinematic measurement errors are large enough, a bias towards One Point models. We expect that more accurate measurement of the vehicle's actual motion would show the original expected preference for Two Point models in the pure translation case.

\subsubsection{Case 4: Two Point Contact (Rotation and Translation)}

Figure 8-17 shows that the violation energy based metric does an excellent job of identifying this contact case as a two point contact case. Referring to Figure 8-18 we see that after the initial transient at the beginning of the trial (where the vehicle accelerates from rest to a roughly constant angular velocity) the technique selects either the correct model or the immediately adjacent model (i.e. model $(3,5)$ or model $(3,4))$ in almost every case. As we would expect from the apparent equivalency of the power violation occurs for these two models in Figure 8-9 the technique has difficulty deciding between these two models. We will show in Section 8.8 that these models

are in fact distinct, implying that their apparent equivalency results from limitations on our ability to accurately measure the vehicle data. 

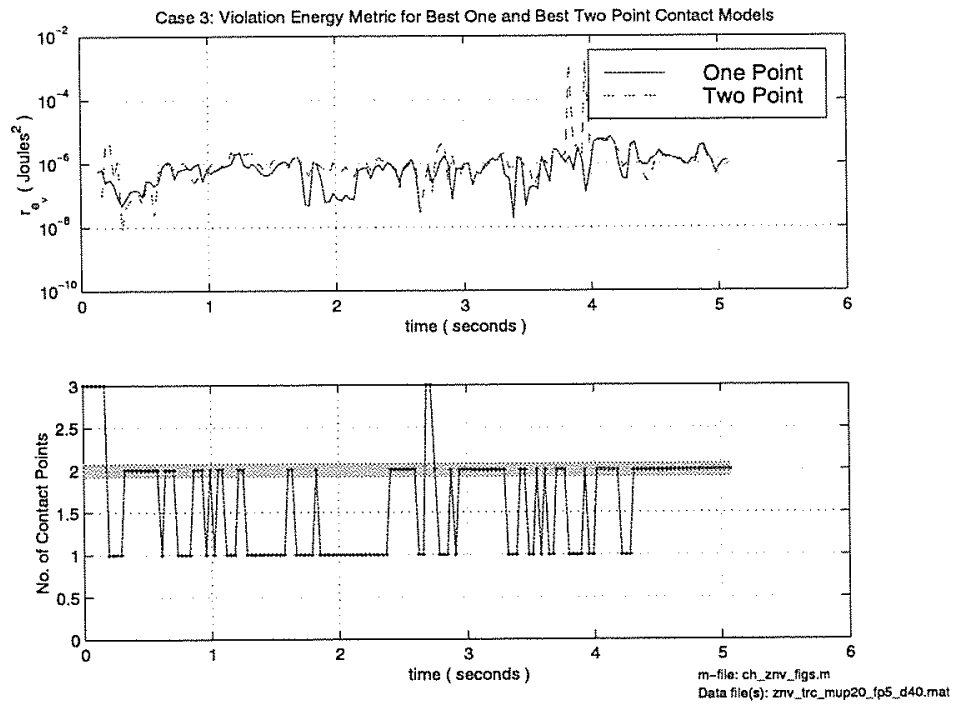

Figure 8-14: Case 3: Violation energy measure $r_{e_{v}}$ the best One Point Contact model and the best Two Point Contact model (upper plot). Number of contact points associated with the best overall model. (lower plot).

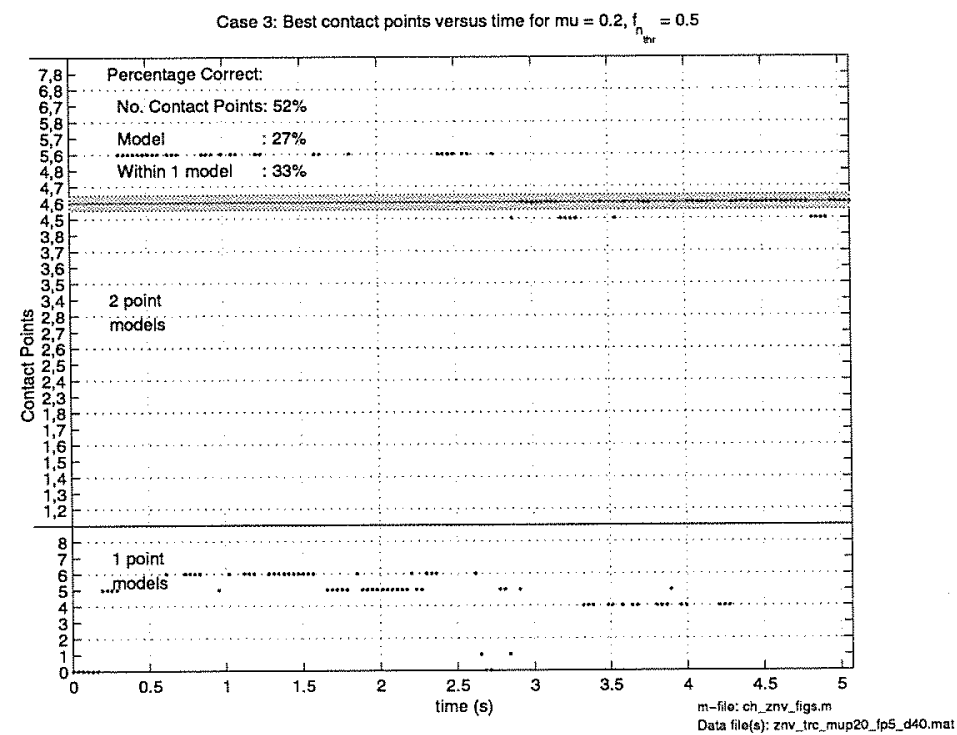

Figure 8-15: Case 3: Best Overall Model vs. Time. 


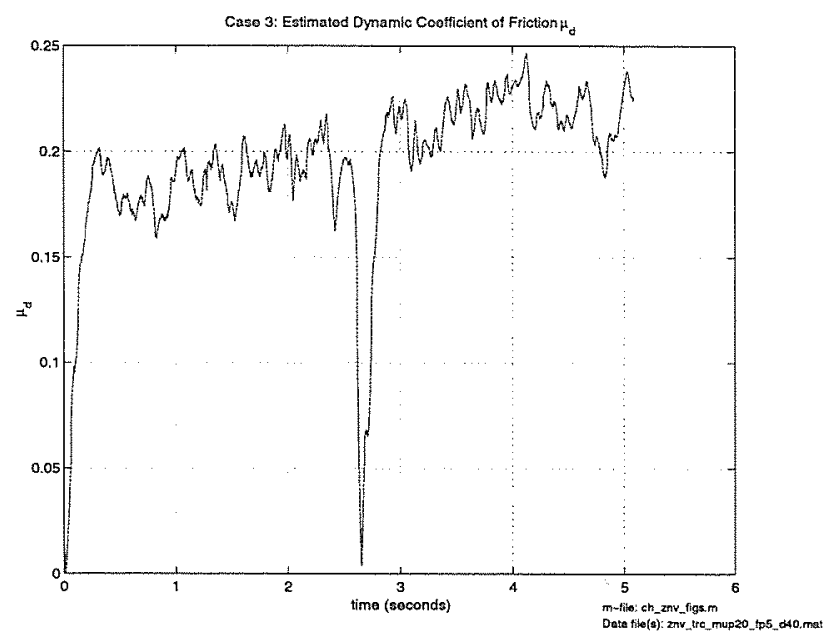

Figure 8-16: Case 3: Estimated coefficient of dynamic friction $\mu_{d}$ during Case 3. $\mu_{d}$ was estimated by dividing the contact force's tangential component by its normal component and taking the absolute value. At time $t=0$ no tangential load is applied to the vehicle and its tangential velocity is still zero. At about time $t=2.7$ seconds the vehicle velocity is reversed. The tangential force briefly goes to zero during the reversal, leading to a second dip in the estimated value of $\mu_{d}$.
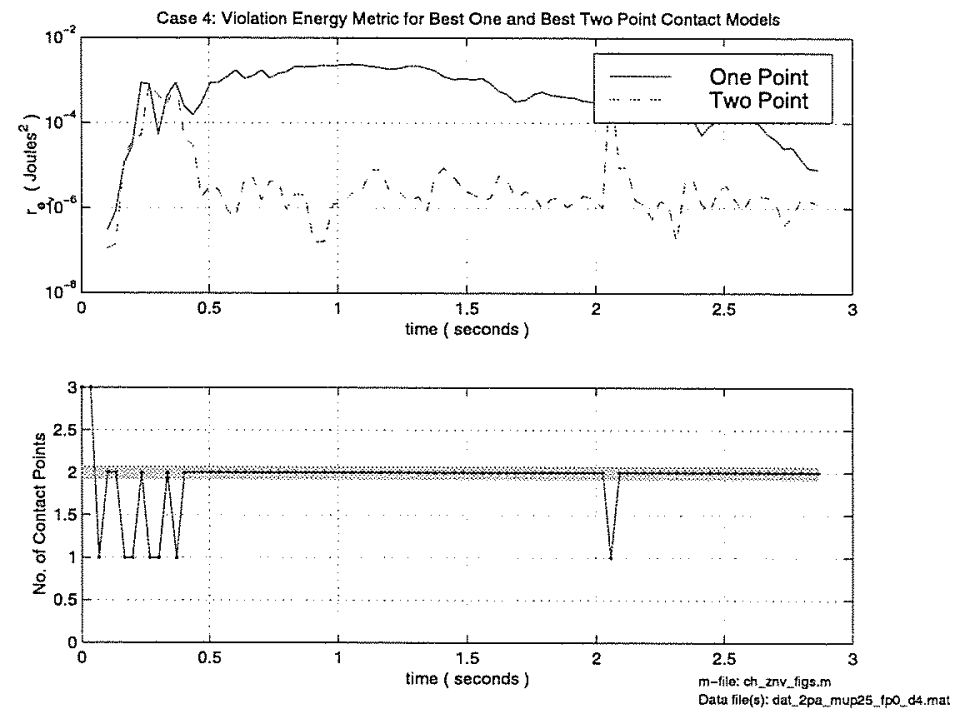

Figure 8-17: Case 4: Violation energy measure $r_{e_{v}}$ the best One Point Contact model and the best Two Point Contact model (upper plot). Number of contact points associated with the best overall model. (lower plot). 


\subsubsection{Case 5: Mixed Contact}

In this trial the vehicle undergoes several transitions in contact state as shown in Figure 8-19. The lower portion of Figure 8-20 shows the actual number of contact points (shaded region) and the number of contact points associated with the best overall model (dots). While not in perfect agreement, the vast majority of the selected models have the correct number of contact points. Referring to Figure 8-21 we see that the technique selects the correct model or an immediately adjacent model the majority ( $78 \%$ ) of the time. For comparison, given that we have 38 possible contact models, we would expect a random selection process to yield a correct result only 2.6 percent of the time.

\subsection{Hazards of Inequality Based Tests}

A potential weakness of the approach presented in this basis is its reliance on inequality based tests to determine the feasibility of candidate models. Such tests are used at three points in the technique. We discuss each briefly in this section.

\subsubsection{Quasistatic Feasibility Test}

To determine a model's quasistatic feasibility we solve for the reaction loads at its presumed contact points and test to see if the normal components at each point are all less than zero. When the magnitude of a computed normal force is close to zero, small errors in the computed value can lead to the spurious exclusion or inclusion of the associated model from/into the set of feasible candidate contact models. This can lead to the selection of the wrong contact model. 

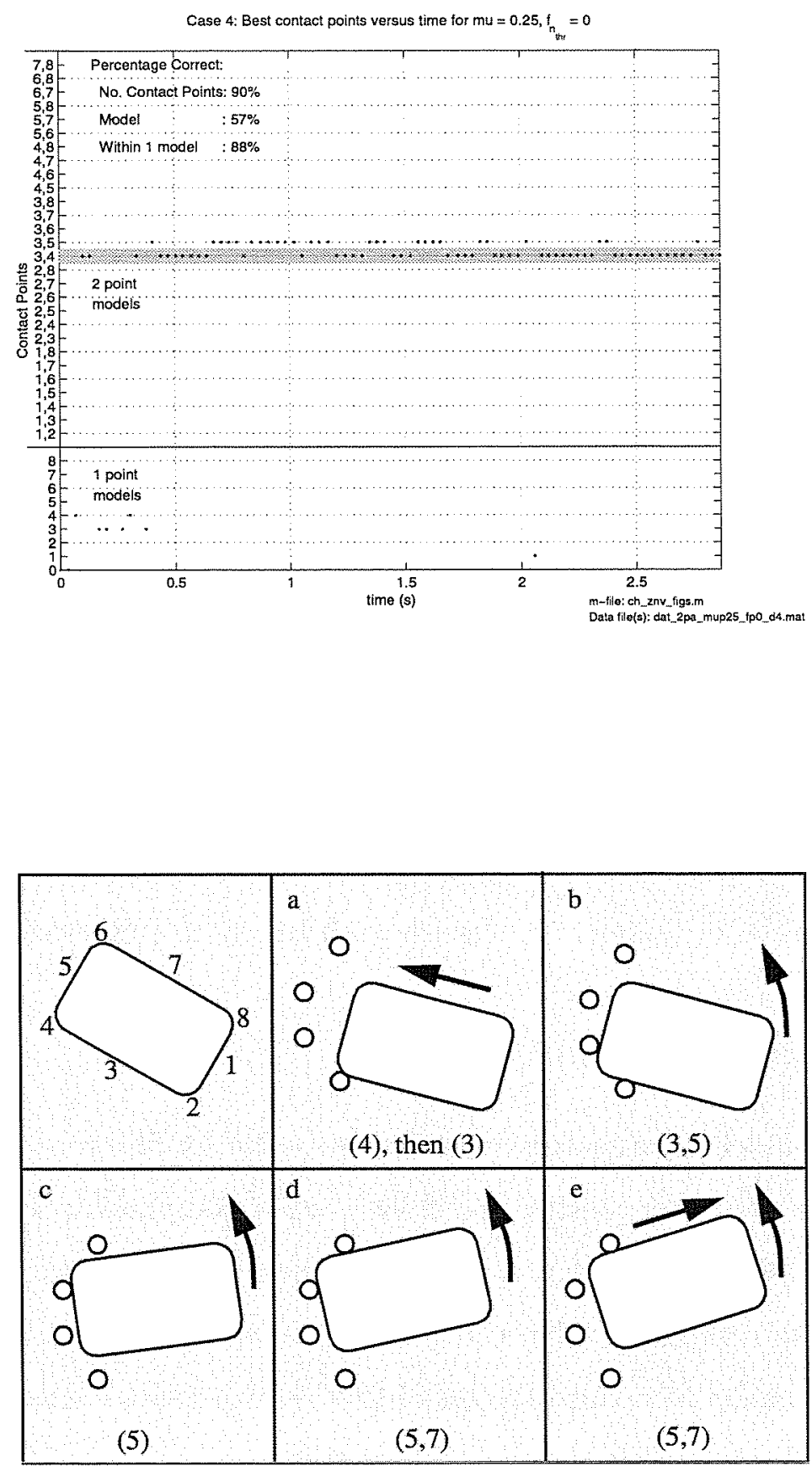

Figure 8-19: Case 5 Experiment: The vehicle was brought into contact with a set of four fixed cylindrical obstacles. The vehicle was moved such that the sequence of contact states depicted above took place. All four obstacles were $12.7 \mathrm{~mm}$ diameter stainless steel bars. 

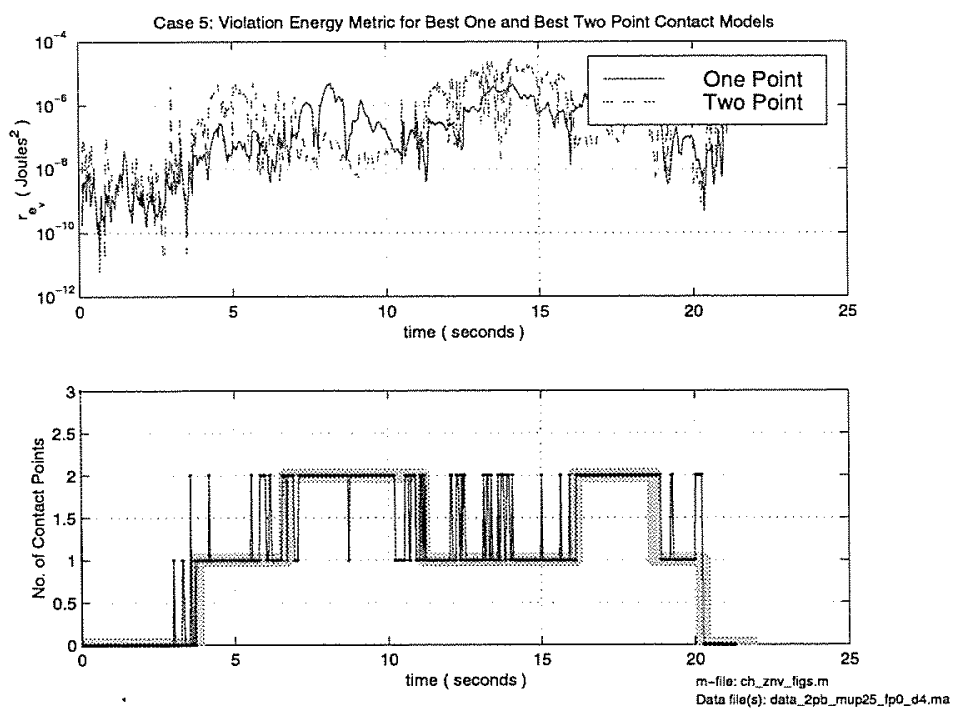

Figure 8-20: Case 5: Violation energy measure $r_{e_{n}}$ the best One Point Contact model and the best Two Point Contact model (upper plot). Number of contact points associated with the best overall model. (lower plot).

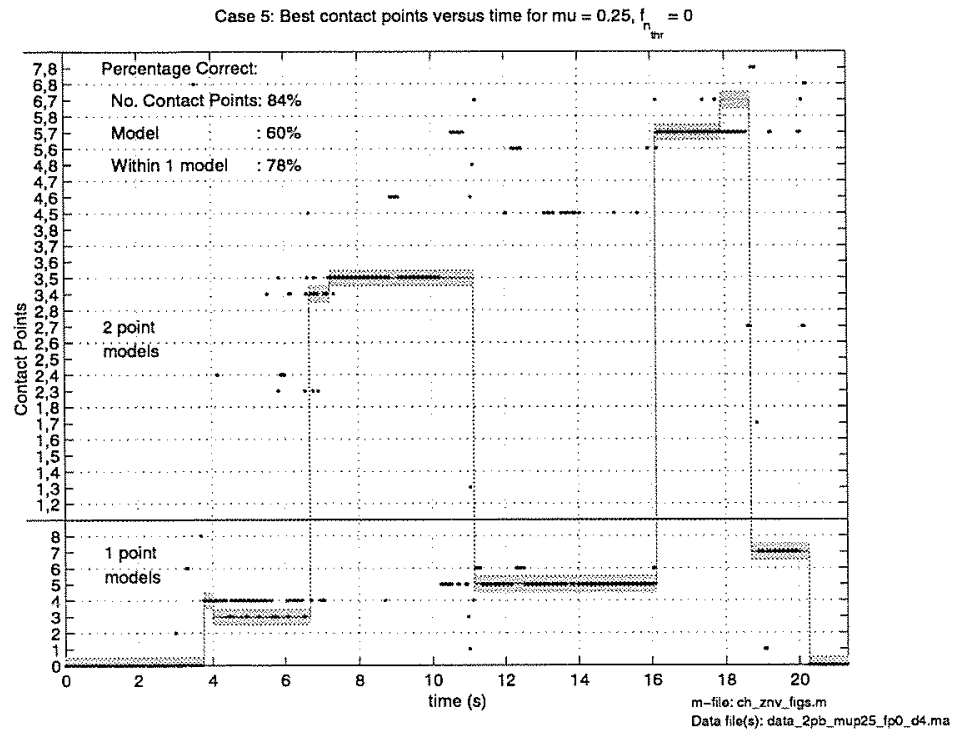

Figure 8-21: Case 5: Best Overall Model vs. Time. 


\subsubsection{Direction of Friction Force Test}

To determine the direction of the friction force acting at each of a model's presumed contact points we determine the sign each contact point's computed tangential velocity. When the tangential velocity is close to zero, small errors in its computed value cause us to assign the wrong direction to the friction force which can have a dramatic effect on the model's computed reaction loads. This in turn can cause the correct model to be interpreted as infeasible or, conversely, to an infeasible model being interpreted as feasible.

\subsubsection{Candidate Contact Point Domain Test}

Similar problems arise in the identification of the candidate contact points. Recall that we find these points by computing the zero-normal-velocity (znv) point for each boundary segment on the vehicle. Regardless of the vehicle velocity, we can always find at least one such point for every boundary segment in the vehicle. The set of candidate contact points is the set of znv points which reside within the domain of their associated boundary segment's. When a znv point occurs close to the end point of a boundary segment, small errors in the znv point's computed location can once again lead to the spurious exclusion or inclusion of the boundary segment's associated candidate contact models.

\subsection{Sensitivity to Assumed Value of Dynamic Co- efficient of Friction}

A potential weakness of the identification method is the assumption that we know the dynamic coefficient of friction $\mu_{d}$. We will investigate the sensitivity using the one point with slip data set as the results for this case appear to be more sensitive than the other trials. We shall roughly gage the sensitivity of the technique to errors in 


\begin{tabular}{|r|r|r|r|}
\hline$\mu_{d}$ & No. Points. & Best Model & Best Model or Adj. Model \\
\hline 0.00 & $18 \%$ & $9 \%$ & $17 \%$ \\
\hline 0.05 & $14 \%$ & $7 \%$ & $12 \%$ \\
\hline 0.15 & $23 \%$ & $14 \%$ & $20 \%$ \\
\hline 0.20 & $56 \%$ & $44 \%$ & $52 \%$ \\
\hline 0.25 & $78 \%$ & $61 \%$ & $68 \%$ \\
\hline 0.30 & $71 \%$ & $59 \%$ & $67 \%$ \\
\hline 0.35 & $59 \%$ & $45 \%$ & $53 \%$ \\
\hline
\end{tabular}

Table 8.1: Percentage of time the technique selected 1) the correct number of contact points, 2) the best overall model and 3 ) the best overall model or an immediately adjacent model.

$\mu_{d}$ by comparing the percentage of the time the technique selects the correct number of contact points, 1) the correct number of contact points, 2) the best overall model and 3) the best overall model or an immediately adjacent model for different values of $\mu_{d}$. These results are tabulated in Table 8.1. The actual coefficient of friction was independently estimated to be in the range $0.20 \leq \mu_{d} \leq 0.25$.

These results imply that the technique is sensitive to errors in the assumed value of the coefficient of friction. The best results occur for $0.20 \leq \mu_{d} \leq 0.30$ which is in reasonably good agreement with the estimated value for $\mu_{d}$.

\subsection{Numerical Investigation of the Uniqueness of Apparently Equivalent Models}

In Section 8.4.2 we found a pair of Two Point Contact models that produced nearly identical values for the violation power based consistency measure $r_{p_{v}}$. This raises the question of whether the models are equivalent or not. Given the way we determine $r_{p_{v}}$, the models could only be equivalent (i.e. could only produce identical $r_{p_{v}}$ values) if and only if their permissible force spaces are identical (i.e. if the spaces span the same vector subspace) at every time point in the data set. 


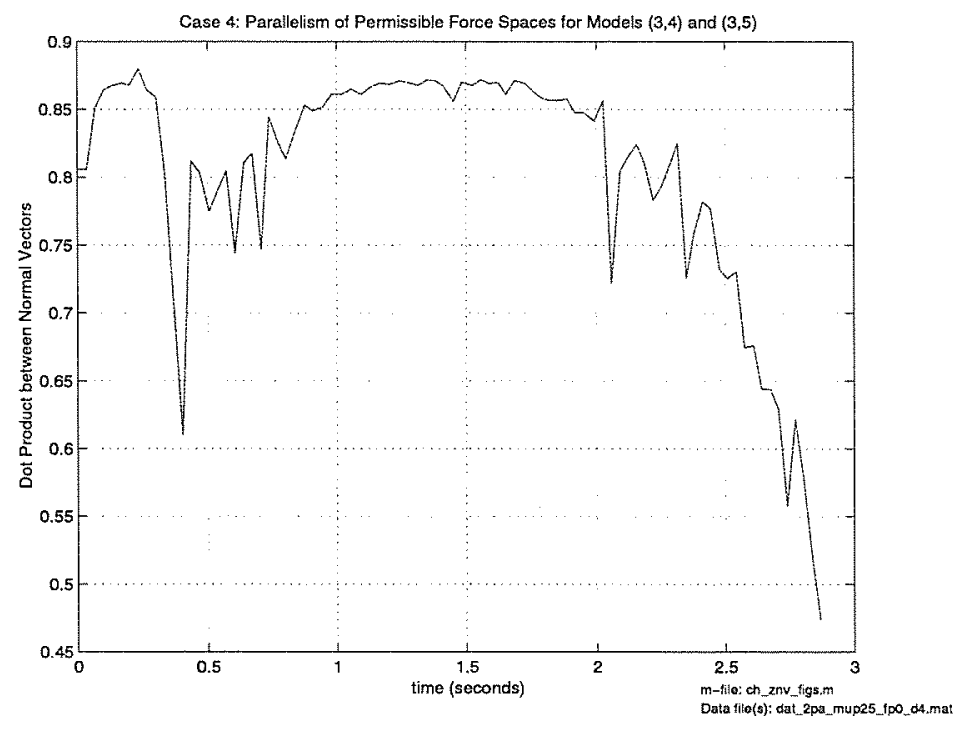

Figure 8-22: Violation power metric $r_{p_{v}}$ for Case 4i.e. for

For any Two Point Contact model, the permissible force space is a two dimensional subset (i.e. a plane) within the three dimensional space of possible measured force vectors. The permissible force spaces of two Two Point Contact models will be identical if they produce permissible force space/ planes which are parallel to each other. We can test for parallelism between the two planes by examining the scalar product of their respective unit normal vectors equals. Permissible force spaces which are parallel should produce a scalar product equal to plus or minus one while orthogonal spaces should produce a scalar product equal to zero. Thus if the two models from Section 8.4.2 are equivalent we expect them to consistently produce scalar products close to magnitude one.

Figure 8-22 shows this scalar product for models $(3,4)$ and $(3,5)$ for the data set presented in Section 8.4.2. Intuitively we expect models $(3,4)$ and $(3,5)$ to be identical when the candidate contact points for arc 4 and edge 5 coincide, i.e. when one of the actual contact points coincides with the intersection point between arc 4 and edge 5 . This situation occurs at about $t=1.58$ seconds in the trial and we see 
from Figure 8-22 that this is indeed when the two models' permisible force spaces are most similar. For times to either side of $t=1.58$ seconds we see that the force spaces become progressively more distinct. This is reflected in the lower portion of Figure 8-9. For times $0.75 \geq t \leq 1.20$ seconds the technique most frequently selects the correct model $(3,5)$. For times near the transition point $(1.20 \geq t \leq 1.90)$ the technique selects both models with almost equal frquency. At the end of the trial, the technique almost exclusively selects the new correct model $(3,4)$. (We have ignored the results in the first 0.5 seconds of the trial because the vehicle experiences significant acceleration during this time as it starts from rest and is rapidly brought up to speed. Thus the quasistatic assumption does not hold in this region and the results produced by the technique are not necessairly reliable. By $t \approx 0.5$ seconds the startup transient is largely over).

Since the models are distinct, we conclude that the difficulty in distinguishing between these two models is not due to the fundamental equivalence of the models but rather to the quality of our sensor data and our knowledge of the physical parameters of the system (i.e. the geometry of the vehicle boundary and, more importantly, the assumed value of the coefficient of friction $\mu_{d}$ ).

\subsection{Conclusion}

This chapter presented experimental results which confirm the efficacy of the contact identification technique presented in the previous chapters. A variety of contact scenarios were investigated, including one point contact with no slip, one point contact with slip, two point contact with rotation and translation, two point contact with pure translation and mixed contact involving multiple transitions between single point contact, two point contact and no contact. In each case the technique selected either the correct model or a reasonable, representative model the majority of time. 


\section{Chapter 9}

\section{Conclusions}

This thesis presented two tools to improve the robustness of manipulation systems to geometric uncertainty when operating in poorly characterized (i.e unstructured ) task environments.

\subsection{Part I: Misalignment Tolerant Grasping}

\subsubsection{Summary}

Part I of this work concentrated on minimizing a manipulation system's sensitivity to positioning errors between the manipulator end effector and task objects. A simple, novel grasping system was presented, consisting of a gripper and a suite of compatible handles which passively self align relative to the gripper when grasped. Each handle imposes a different degree of alignment and also exhibits a different actuatororthogonal force space, i.e. a vector space of applied loads whose support requires no actuator torque. Since its integration into the manipulation subsystem the Jason Remotely Operated Undersea Vehicle (operated by the Woods Hole Oceanographic Institution) the grasping system has been used extensively to perform a wide range of ocean science tasks at depths as great as 5000 meters. These tasks include tool based tasks (e.g. sediment sampling, hydrothermal vent water sampling, mating 


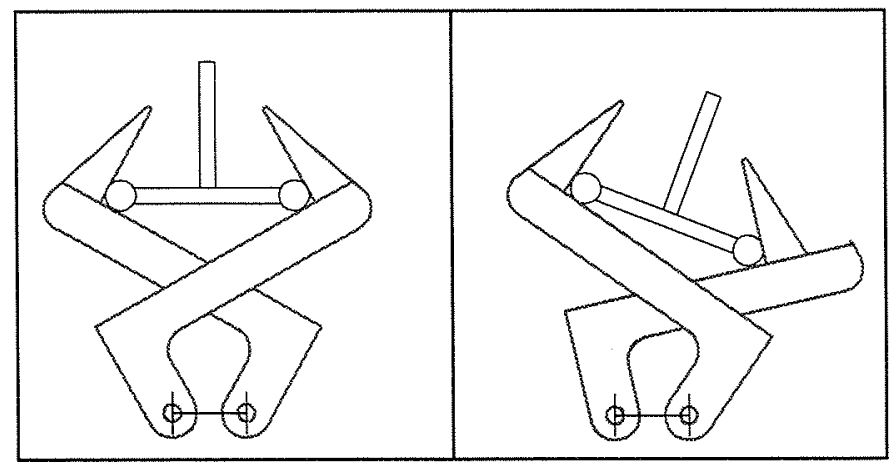

Figure 9-1: Example of a gripper with variable geometry fingers dexterously manipulating a handle.

and de-mating electrical connectors), tasks involving unstructured objects (e.g. sampling mussels and tube worms, acquiring rock samples) and a great many equipment transfer tasks (e.g. loading and unloading sampling devices, samples and sample containers).

\subsubsection{Future Work}

\section{Dexterous Manipulation of Handles}

The geometry of a handle, in conjunction with that of the gripper fingers, determines the ultimate alignment of the handle when fully grapsed. By designing a gripper which has variable finger geometry, the position and orientation of a grasped handle could be varied continuously by commanding coordinated changes to the geometry of the different fingers (see Figure 9-1). This would represent a fundamentally new type of dexterous grasping. Typical dexterous grasping relies entirely on friction forces to constrain a grasped object and therefore is limited to tasks involving small manipulation loads. Slip between the gripper and grasped object is vigorously avoided. Constraint of handles, even in the dexterous case, relies on geometric constraint and would therefore be capable of handling much higher manipulation loads. Slip in this 
type of grasping is not only desirable, but required. As such, the handles and fingers should be designed to have as low a coefficient of friction as possible.

Such a gripper/handle system would be an implementation of the macro-micro manipulator concept. Key issues would be manipulability of the handle, avoiding jamming and ensuring the ability to apply desired forces and torques to handles. The system could be implemented to actuate all or only a portion of a handle's degreesof-freedom.

\subsection{Part II: Contact Identification}

\subsubsection{Summary}

A technique to identify the contact state of a moving rigid planar body (i.e. the vehicle) interacting with fixed planar bodies (i.e. the environment) was presented and experimentally verified. Given the vehicle's velocity and boundary geometry we solved for the set of kinematically feasible candidate contact points, from which we constructed the set of kinematically feasible candidate contact models. We derived each model's force and velocity constraint equations and showed that they define a model's permissible force and permissible velocity spaces. These vector spaces represent the set of possible measured vehicle force and velocity vectors which are physically possible given the constraints of the assumed model.

Using each model's permissible force and velocity spaces we decomposed the actual measured force and velocity into permissible and impermissible components. The impermissible components represent the degree to which the current measured data violate a given model's constraint equations. From these components we computed the violation power, i.e. the power dissipation associated with violation of each of a model's constraint equations. By properly combining the individual violation power terms for each constraint equation we formed a positive definite consistency measure by which we assessed the degree of compatibility between each candidate model and 
the current measured data vectors.

The violation power based metric, however, only identified the best model within a model class. To identify which of the best-of-class models is the best overall model, we introduced a similar metric based on the violation energy, i.e the work associated with the violation of a models constraint equations during a given incremental motion of the vehicle. Finally, we demonstrated experimentally the efficacy of this two stage identification process.

\subsubsection{Future Work}

\section{Applicability to the General Manipulation Problem}

While this work has been presented in the context of a vehicle interacting with its environment, the identification technique applies equally well to the problem of a manipulator end effector or grasped object being manipulated in an environment populated by fixed objects. Thus the technique can be applied to a wide range of compliant motion control problems. Example uses could include detecting contact transitions during automated assembly for disassembly tasks or enabling a manipulator to autonomously explore an unknown local environment.

\section{Improving Identification Accuracy}

While the technique performs well, the accuracy must be improved to ensure reliable performance of a complete manipulation system.

The most straightforward path to improved performance is the use of better sen-

sors. For the experiments presented, availability dictated the use of a force/torque sensor designed to measure loads 10 to 50 times larger than the typical loads encountered in these experiments. In addition, more up-to-date force/torque sensors incorporate accelerometers to enable the subtraction of acceleration induced loads from the sensor signal. Use of a properly scaled, acceleration compensated force 
sensor could substantially improve the quality of the force and torque measurements.

Likewise, the accuracy of the velocity measurement technique employed becomes quite poor near zero velocity. While zero crossing will ultimately be a problem regardless of sensor resolution, a more accurate velocity sensor would yield improved performance for a wider range of vehicle conditions.

As mentioned earlier, no filtering of the force or velocity data was performed for any of the experiments presented in this thesis. Any introduction of filtering must be carefully considered due to the fact that the technique is based on the relationship between the instantaneous measured force and the instantaneous measured velocity. Thus, it is advisable that the two measurements should be filtered identically so that they have the same frequency content and lag. A better approach might be to employ smoothing techniques to a block of data centered, say, $t$ seconds in the past to produce zero lag estimates of the force and velocity at this prior time. This approach presents the technique with higher quality estimates of the measured data but yields a contact model which was valid $t$ seconds ago rather than the current model. It may also be possible to integrate the maximum likelihood approach presented by Eberman [9] into technique presented herein.

\section{Incorporation into a Closed Loop Hybrid Controller}

An obvious next step is to incorporate the contact identification system into the closed loop hybrid control of a vehicle or manipulator (see Figure 3-2) to perform a simple task. In the context of the vehicle control problem, this could be the problem of maintaining vehicle heading while braced against an unknown environment despite the presence of disturbance loads. Alternatively, the vehicle could be used to characterize the unknown environmental geometry by tracking the absolute positions of the actual contact points. 


\subsubsection{Use of the Technique with Concave Objects}

In this thesis we have considered the case of a convex vehicle throughout. None of the arguments presented require that the vehicle be convex. To find the candidate contact points we need to know the vehicle's boundary geometry and velocity, but the concavity or convexity of the vehicle is irrelevant to this portion of the contact identification technique. Once we have the candidate contact points, we can construct

the candidate contact models, again, regardless of the convexity or concavity of the vehicle shape. To select between models we fit the measured force and velocity data to each candidate contact model's permissible force and permissible velocity spaces. Once again, there is no aspect of this fitting process that depends upon the vehicle's convexity. We conclude, therefore, that the contact identification technique presented applies equally well to concave shapes.

\section{Estimation of Dynamic Coefficient of Friction}

The performance of the contact identification technique depends on the accuracy of the assumed value of the coefficient of friction which, in general, will not be well known. The technique would benefit strongly from the incorporation of the ability to estimate the acting coefficient of friction. Ideally this would be a realtime procedure, but could also be obtained from characterization motions performed explicitly for this purpose.

\section{Incorporation into Higher Level Task Planning Systems}

The technique presented in this thesis simply identifies the contact characteristics of the moving vehicle. The problem of intelligently using this information in the control of the vehicle is not a trivial problem, particularly given the fact that the information can at times be incorrect. This area of reasearch presents many unsolved challenges. 


\section{Extension to 3-D Systems}

Fundamentally the technique seems extensible to the three-dimensional case. For example, given a three dimensional body's surface geometry and linear and angular velocities we can solve for the set of kinematically feasible candidate contact points. Similarly, we can use these points to construct the set of kinematically feasible contact models and their permissible force and velocity spaces. Given these spaces, we should still be able to decompose the measured velocity and force into permissible and impermissible components and, therefore, we should be able to compute the violation power and energy consistency metrics.

The number of candidate contact models to be evaluated, however, is apt to be quite large. Given $N$ candidate contact points, one could formulate 1 Unconstrained Model, $N$ unique One Pont Contact Models, $\frac{N}{2 !(N-2) !}$ unique Two Point Contact Models, $\frac{N}{3 !(N-3) !}$ unique Three Point Contact Models, $\frac{N}{4 !(N-4) !}$ unique Four Point Contact Models, $\frac{N}{5 !(N-5) !}$ unique Five Point Contact Models, and 1 Fully Constrained Model. For example, $N=8$ candidate contact points yields 38 posssible planar contact models but yields 220 possible three dimensional contact models. For $N=10$ the number of three dimensional models jumps to 639. The strong dependence on the number of candidate contact points implies that modeling of three dimensional objects is best done using continuous surfaces rather than faceted approximations. The former representation yields far fewer candidate points and therefore, far fewer associated candidate contact models requiring assessment.

\subsection{Conclusion}

This thesis presented two new tools which improve a manipulator's ability to cope with uncertainty in its position and orientation relative to objects in the environment. The first tool, a new grasping system consisting of a gripper and a suite of compatible handles which passively self align relative to the gripper when grasped, enables a 
manipulator to successfully grasp tools despite significant pre-grasp misalignment (e.g. up to $\pm 5 \mathrm{~cm}$ in some directions) between the gripper and a handle. Since its integration into the Jason Remotely Operated Undersea Vehicle (operated by the Woods Hole Oceanographic Institution) the system has been used extensively to perform a wide variety of deep ocean science tasks.

The second tool, a technique to identify the contact state of the moving planar rigid body interacting with fixed planar rigid bodies, can enable the use of existing compliant motion control strategies (e.g. Hybrid Control, Impedance Control) in environments where little or nothing is known about the geometry of environmental objects. Use of this system can improve a vehicle based manipulator's ability to cope with geometric uncertainty in two ways. It can be used in the direct control of the manipulator as it interacts with the unknown environment and, if integrated to the control of the vehicle, can enable the vehicle to stabilize itself against manipulation reaction loads by bracing itself against the unknown environment. This thesis experimentally validated the efficacy of the contact identification technique. Future work includes the incorporation of this technique into the closed loop hybrid control of a manipulator or vehicle system. 


\section{Appendix A}

\section{Experimental Apparatus}

This appendix describes the laboratory apparatus used to experimentally validate the proposed contact accommodation scheme. The issues driving the design of the system are discussed as are the system's physical characteristics, including dimensional scaling of the mechanism and the mechanism's kinematic, static and dynamic properties.

\section{A.1 Justification for building an experimental testbed}

The following paragraphs summarize possible platforms that could be used to test the contact identification scheme along with the rationale for their selection or rejection.

Control a real underwater vehicle: Use of a real ROV system presents significant logistical problems including limited availability for experiments ( due to high demand for their services ), expense of operation ( due to the specialized facilities and personnel required for their operation ) and the risk the experiments pose to the vehicle system. Little quantitative data exists for many important system parameters (e.g thruster bandwidth during thrust reversal ) while variation of better known physical characteristics is impractical (e.g. effective mass, thruster mounting geometry ). Tests using a real ROV would limit conlusions to "it worked with this system" or "it 
didn't work with this sytem" with little hope in either outcome of learning why.

Control a simulated underwater vehicle: Accurately simulating the dynamics of rigid and/or compliant bodies in impact situations involving friction is a complex task which is still an active area of research. Most of the existing approaches greatly simplify or ignore the detailed dynamics which occur during impact events. Since such effects are likely to present the greatest challenge to successful closed loop control during contact, simulation alone cannot adequately validate the technique presented in this thesis.

Control a dimensionally similar physical system: While time consuming to develop, this approach avoids the logistical and quantification problems associated with using a real system while ensuring the presence of impact related dynamic effects that would be ignored by or poorly modeled by a simulated vehicle.

The remainder of this appendix discusses the design and characteristics of the dimensionally scaled apparatus constructed for the validation experiments described in Chapters 4 and 8.

\section{A.2 Form of the Apparatus}

We have many options when considering how to construct an experimental system. Table A.2 lists some of the more promising options for each of the four system functions listed, where we have underlined the options chosen for use in our system.

A-1 shows a schematic of the proposed system. Four stationery, brushless D.C. motors control the three planar D.O.F. of the model vehicle $(x, y$ position and angular orientation) via mechanical cables connecting the motor pulleys to the a pulley on the vehicle (Applying arbitrary force / torque combinations to the model vehicle using three motors would require some cables to "push" on the vehicle. Adding a fourth motor guarantees that arbitrary force / torque combinations can be applied to the model vehicle using only "pulling" or tensile cable loads). The vehicle boundary 


\begin{tabular}{|c||c||c||c|}
\hline Support Mechanism & Actuation & Position Sensing & Force Sensing \\
\hline Buoyancy & Propeller & Acoustic & Instrumented Bumper \\
Air Bearing & Air Jet & Encoder Arm & Force/Torque Sensor \\
Water Bearing & Water Jet & Encoder/Cables & \\
SCARA Arm & Motor/Cables & \\
Sliding Bearing & SCARA Arm & & \\
& Planar Motor & & \\
& & & \\
\hline
\end{tabular}

Table A.1: Selected implementation options for the primary system functions.

\begin{tabular}{|l|l|}
\hline Scaling & Electronics \\
Forward Kinematics & Inverse Kinematics \\
Jacobian & Inertia Variations \\
Cable Tensioning & Motor Friction \\
Position Sensing & Force Sensing \\
Velocity Sensing & Torque Ripple \\
Air Bearing Design & Motor Drivers \\
Impact Behavior & Controller \\
\hline
\end{tabular}

Table A.2: Issues involved in the design of the experimental apparatus

plate (the part of the model vehicle which makes contact with the environment) is connected to the vehicle pulley via a 6 axis force torque, enabling the measurement of forces and moments resulting from contact with the environment. Three air bearing pads support the model vehicle, enabling it to move freely on the table surface with the static friction in the system coming from the bearing friction associated with the brushless motors. Since the drive mechanism is essentially a direct drive (i.e. there is no reduction between the motor and its pulley and the motor and vehicle pulleys are the same diameter) the contribution of the motors and motor pulleys to the total vehicle inertia can be made quite small, producing a system which closely approximates the inertia characteristics of the real vehicle. We shall discuss the design particulars in greater detail later in this chapter. 


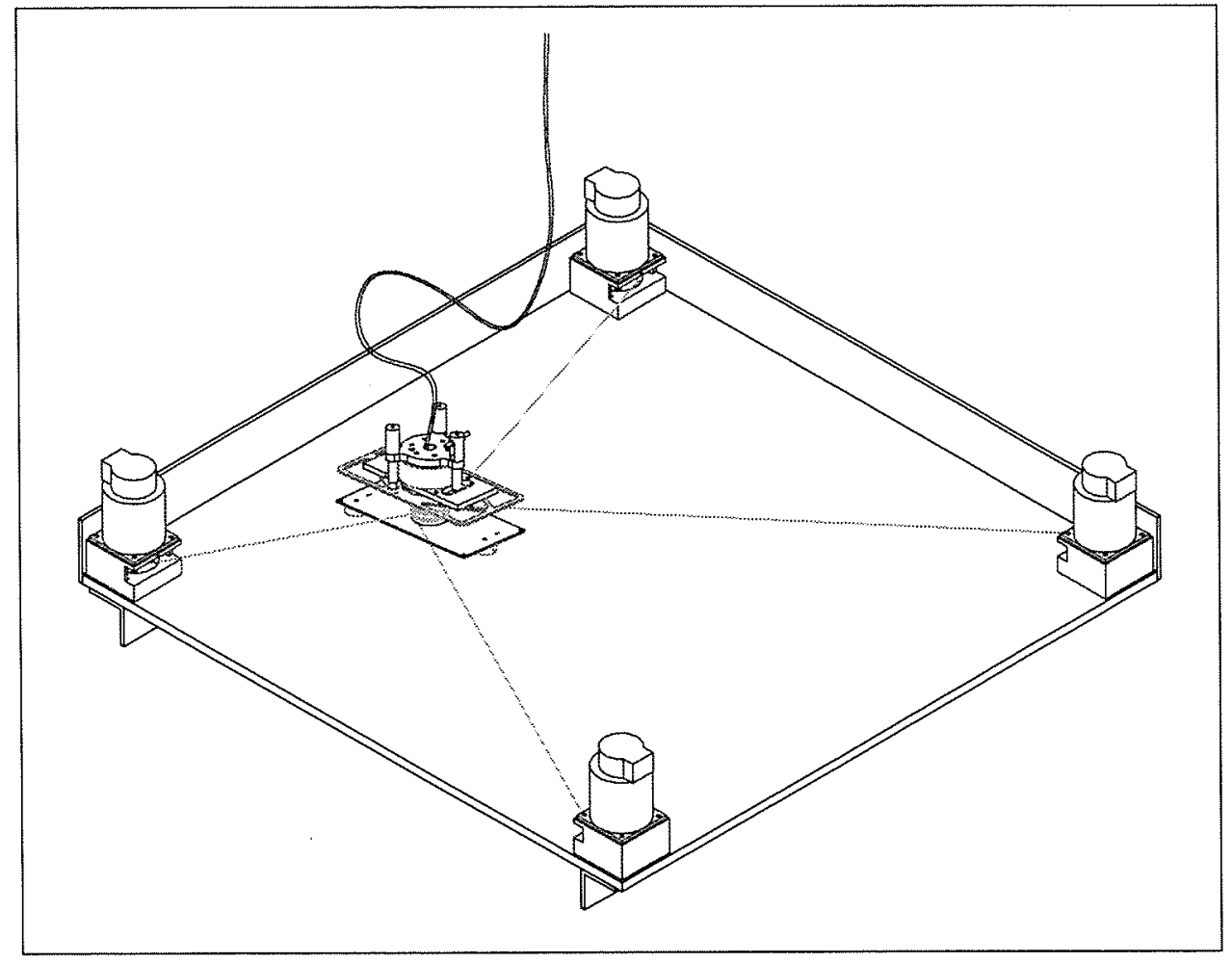

Figure A-1: Planar vehicle testbed 


\section{A.3 Scaling}

Our experiments are of no use unless 1) the conditions we subject the model vehicle to correspond in a known way to those we expect the full size vehicle to encounter and 2) the response of the model vehicle to these model conditions corresponds in a known way to response the full sized vehicle would have if subjected to the corresponding "full size" conditions. The appropriate way to determine these relationships is through dimensional analysis. In this approach we transform a system's governing equations, which relate dimensioned, physical paramters (e.g. length, mass and velocity of a vehicle) into a dimensionless or unit-less form which involves only dimensionless ratios of these physical parameters. In this form, the governing equations for two different physical systems will be identical if these dimensionless ratios are the same for both systems. It is from these dimensionless ratios that we determine how to size our experimental system and its associated conditions to accurately model the behaviour of a full sized vehicle.

\section{A.3.1 Dimensional Analysis}

The first step in dimensional analysis is to determine a complete and independent set of quantities on which the dependent variables in the governing equations depend. For our situation ( a planar vehicle) the gsfoverning equations for any possible contact configuration are the three equilibrium equations obtained by setting the sums of the $x$ and $y$ forces (including D'Alembert forces) and the $z$ moments acting on the vehicle to zero (where $x, y$ and $z$ form a right handed axis system whose $x$ and $y$ axes lie in the plane in which the vehicle moves). Generically, these equations are of the form

$$
\begin{aligned}
& F_{x}=F_{x}\left(m, d_{1}, \ldots, d_{l}, \phi_{1}, \ldots, \phi_{m}, x, \dot{x}, \ddot{x}, \alpha, \dot{\alpha}, \ddot{\alpha} F_{N_{1}}, \ldots, F_{N_{n}}, \mu_{1}, \ldots, \mu_{n}\right) \\
& F_{y}=F_{y}\left(m, d_{1}, \ldots, d_{l}, \phi_{1}, \ldots, \phi_{m}, y, \dot{y}, \ddot{y}, \alpha, \dot{\alpha}, \ddot{\alpha}, F_{N_{1}}, \ldots, F_{N_{n}}, \mu_{1}, \ldots, \mu_{n}\right)
\end{aligned}
$$




$$
\tau_{z}=\tau\left(J, m, d_{1}, \ldots, d_{l}, \phi_{1}, \ldots, \phi_{m}, x, \dot{x}, \ddot{x}, y, \dot{y}, \ddot{y}, \alpha, \dot{\alpha}, \ddot{\alpha} F_{N_{1}}, \ldots, F_{N_{n}}, \mu_{1}, \ldots, \mu_{n}\right)
$$

where $m$ is the vehicle mass, $\left(d_{1}, \ldots, d_{l}\right)$ and $\left(\phi_{1}, \ldots, \phi_{m}\right)$ are defining linear and angular geometric values, $(x, \dot{x}, \ddot{x})$ and $(y, \dot{y}, \ddot{y})$ are the position, velocity and acceleration of the vehicle center of mass, $(\alpha, \dot{\alpha}, \ddot{\alpha})$ are the angular orientation, rate and acceleration of the vehicle about the $z$ axis, $\left(F_{N_{1}}, \ldots, F_{N_{n}}\right)$ are the contact normal forces acting on the vehicle and $\left(\mu_{1}, \ldots, \mu_{n}\right)$ are the coefficients of friction associated with these contacts. Referring to the equations of motion derived in the simulation section for the various planar contact configurations we see that this set is complete and independent (Note, however, that for a given contact case the contribution of many of these terms is zero, e.g. a vehicle constrained in the $x$ direction will have zero contribution from terms involving $\dot{x}$ and $\ddot{x}$ ).

Following this, we consider the dimensions of the dependent and independent quantities involved in the governing equations. These are

$$
\begin{aligned}
{\left[F_{x}\right] } & =\frac{M L}{T^{2}} & {[x] } & =L \\
{\left[F_{y}\right] } & =\frac{M L}{T^{2}} & {[\dot{x}] } & =\frac{L}{T} \\
{\left[\tau_{z}\right] } & =\frac{M L^{2}}{T^{2}} & {[\ddot{x}] } & =\frac{L}{T^{2}} \\
{[J] } & =M L^{2} & {[\dot{\alpha}] } & =\frac{1}{T} \\
{[m] } & =M & {[\ddot{\alpha}] } & =\frac{1}{T^{2}} \\
{\left[d_{1}\right], \ldots,\left[d_{l}\right] } & =L & {\left[F_{N_{1}}, \ldots, F_{N_{n}}\right] } & =\frac{M L}{T^{2}} \\
{\left[\phi_{1}\right], \ldots,\left[\phi_{m}\right] } & =1 & {\left[\mu_{1}, \ldots, \mu_{n}\right] } & =1
\end{aligned}
$$

where the notation $a[\beta]$ means "the dimension of $\beta$ " and $M, L$ and $T$ denote the fundamental dimensions of mass, length and time respectively.

From the independent variables we select a complete, dimensionally independent subset of variables. This is a subset of the variables which can be combined in terms of products and powers to produce terms which are dimensionally equivalent to the 
remaining members of the original set but which cannot be so combined as to produce terms which are dimensionally equivalent to each other. For our analysis we select the vehicle mass $m$, the geometric length $d_{1}$ which we shall define as the length of the vehicle and the vehicle angular rate $\dot{\alpha}$ as our dimnesionally independent subset of variables. Using these we may define the following dimensionless forms of the original terms, where the notation $\beta^{*}$ denotes the dimensionless form of $\beta$.

$$
\begin{aligned}
F_{x}^{*} & =\frac{F_{x}}{m d_{1} \dot{\alpha}^{2}} & \ddot{x}^{*} & =\frac{\ddot{x}}{d_{1} \dot{\alpha}^{2}} \\
F_{y}^{*} & =\frac{F_{y}}{m d_{1} \dot{\alpha}^{2}} & y^{*} & =\frac{y}{d_{1}} \\
\tau^{*} & =\frac{\tau}{m d_{1}^{2} \dot{\alpha}^{2}} & \dot{y}^{*} & =\frac{\dot{y}}{d_{1} \dot{\alpha}} \\
J^{*} & =\frac{J}{m d_{1}^{2}} & \ddot{y}^{*} & =\frac{\ddot{y}}{d_{1} \dot{\alpha}^{2}} \\
m^{*} & =1 & \alpha^{*} & =\alpha \\
d_{i}^{*} & =\frac{d_{i}}{d_{1}} & \dot{\alpha}^{*} & =1 \\
\phi_{i}^{*} & =\phi_{i} & \ddot{\alpha}^{*} & =\frac{\ddot{\alpha}}{\dot{\alpha}^{2}} \\
x^{*} & =\frac{x}{d_{1}} & F_{N_{i}}^{*} & =\frac{F_{N_{i}}}{m d_{1} \dot{\alpha}^{2}} \\
\dot{x}^{*} & =\frac{\dot{x}}{d_{1} \dot{\alpha}} & \mu^{*} & =\mu
\end{aligned}
$$

Thus to ensure that our model vehicle accurately mimics the full size vehicle we must scale our model such that these dimensionless ratios have the same value for both systems, i.e. that

$$
\beta_{\text {model }}^{*}=\beta_{\text {real }}^{*}
$$

where $\beta$ is the dimensionless ratio in question. Our selection of the dimensionally independent subset guarantees that $m^{*}$ and $\dot{\alpha}^{*}$ are equal for all systems. Requiring that $d_{i}^{*}$ be the same for both systems is most easily satisfied by uniformly scaling the geometry of the real vehicle/environment by a constant factor to obtain the model 
system geometry. To see this we equate a given $d_{i}^{*}$ for the two cases, obtaining

$$
\begin{aligned}
d_{i_{\text {model }}}^{*} & =d_{i_{\text {real }}}^{*} \\
\left(\frac{d_{i_{\text {model }}}}{d_{1_{\text {model }}}}\right) & =\left(\frac{d_{i_{\text {real }}}}{d_{1_{\text {real }}}}\right) \\
\left(\frac{d_{i_{\text {model }}}}{d_{i_{\text {real }}}}\right) & =\left(\frac{d_{1_{\text {model }}}}{d_{1_{\text {real }}}}\right) \equiv K_{l} \\
& =\text { constant }
\end{aligned}
$$

A similar analysis reveals the same scale factor relates the $x$ and $y$ cordinates of the vehicle center of mass for the two systems. Strictly speaking, we only need to use this scale factor for those dimensions which actually show up in the equations of motion. Since any geometric dimension of the vehicle's planar boundary can be involved in the dynamics of some contact configuration, we should scale all of the vehicle dimensions by this same factor.

A consequence of uniformaly scaling the linear geometry of the vehicle is that all angles in the model system will be the same as the corresponding angles in the real system. Thus uniformly scaling the vehicle and environmental geometry ensures that $\alpha^{*}$ and the $\phi_{i}^{*}$ will also be equal for the two systems.

Applying equation A.4 to the dimensionless linear velocities $\dot{x}^{*}$ and $\dot{y}^{*}$ we find that

$$
\frac{\dot{x}_{\text {model }}}{\dot{x}_{\text {real }}}=\frac{\dot{y}_{\text {model }}}{\dot{y}_{\text {real }}}=\frac{d_{1_{\text {model }}}}{d_{1_{\text {real }}}} \frac{\dot{\alpha}_{\text {model }}}{\dot{\alpha}_{\text {real }}}=K_{l} K_{\dot{\alpha}} \equiv K_{v}
$$

i.e. the velocity scale factor $K_{v}$ is the product of the scale factors for length and angular velocity $K_{l}$ and $K_{\dot{\alpha}}$. We are free to pick any value we wish for the ratio $K_{\dot{\alpha}}$. We will discuss this choice in more detail momentarily.

Applying equation A.4 to the dimensionless linear and angular accelerations $\ddot{x}^{*}$, $\ddot{y}^{*}$ and $\ddot{\alpha}^{*}$ we find that 


$$
\begin{aligned}
\frac{\ddot{x}_{\text {model }}}{\ddot{x}_{\text {real }}}=\frac{\ddot{y}_{\text {model }}}{\ddot{y}_{\text {real }}} & =K_{l} K_{\dot{\alpha}}^{2} \equiv K_{a} \\
\frac{\ddot{\alpha}_{\text {model }}}{\ddot{\alpha}_{\text {real }}} & =K_{\dot{\alpha}}^{2} \equiv K_{\ddot{\alpha}}
\end{aligned}
$$

Similarly we find that the scale factors for the remaining quantities are

$$
\begin{array}{r}
\frac{F_{x_{\text {model }}}}{F_{x_{\text {real }}}}=\frac{F_{y_{\text {model }}}}{F_{y_{\text {real }}}}=\frac{F_{N_{\text {model }}}}{F_{N_{\text {real }}}}=K_{m} K_{l} K_{\dot{\alpha}}^{2} \equiv K_{f} \\
\frac{\tau_{z_{\text {model }}}}{\tau_{z_{\text {real }}}}=K_{m} K_{l}^{2} K_{\dot{\alpha}}^{2} \equiv K_{\tau} \\
\frac{J_{\text {model }}}{J_{\text {real }}}=K_{m} K_{l}^{2} \equiv K_{J}
\end{array}
$$

where $K_{m}=\frac{m_{\text {model }}}{m_{\text {real }}}$.

Thus we see that by specifying just three of the scale factors (i.e. $K_{l}, K_{m}$ and $K_{\dot{\alpha}}$ ) we specify the scale factors for all of the desired quantities. We are free to select these three scale factors as we please as we design our model system. Before we do this, however, lets consider the scale factor for $K_{\dot{\alpha}}$ more closely.

As noted above, a consequence of uniform scaling of the geometry between the systems is that all corresponding angles in the two systems are equal. Given this, a non-unity value for $K_{\dot{\alpha}}$ (the ratio between the two system's angular velocities) implies a non-unity scale factor for time between the two systems, e.g. a motion of the model vehicle which takes two seconds would represent a geometrically similar motion of the real vehicle that would take, say, 20 seconds. Thus, in selecting $K_{\dot{\alpha}}$ we are really selecting the inverse of scale factor relating the dimension of time in each of systems, i.e. $K_{t}=\frac{t_{\text {model }}}{t_{\text {real }}}=\frac{1}{K_{\dot{\alpha}}}$. Substituting for $K_{\dot{\alpha}}$ we summarize the relationships for the scale factors in Table $\mathrm{xxx}$. 


\begin{tabular}{|l|l||l|l|}
\hline Scale Factor & Value & Scale Factor & Value \\
\hline$K_{l}$ & unconstrained & $K_{\ddot{\alpha}}$ & $\frac{1}{K_{t}^{2}}$ \\
$K_{m}$ & unconstrained & $K_{J}$ & $K_{m} K_{l}^{2}$ \\
$K_{t}$ & unconstrained & $K_{f}$ & $\frac{K_{m} K_{l}}{K_{t}^{2}}$ \\
$K_{v}$ & $\frac{K_{l}}{K_{t}}$ & $K_{\tau}$ & $\frac{K_{m} K_{l}^{2}}{K_{t}^{2}}$ \\
$K_{a}$ & $\frac{K_{l}}{K_{t}^{2-2}}$ & $K_{\mu}$ & 1 \\
$K_{\alpha}$ & 1 & $K_{\dot{\alpha}}$ & $\frac{1}{K_{t}}$ \\
$K_{\ddot{\alpha}}$ & $\frac{1}{K_{t}^{-2}}$ & $K_{k}$ & $\frac{K_{m}}{K_{t}^{2}}$ \\
\hline
\end{tabular}

\section{A.3.2 Parameter values for the Jason ROV}

To employ the scaling laws we need to know the pertainant characteristics of the real vehicle. Table xxx summaraizes these characteristics for the Jason ROV operated by the Deep Submergence Lab of the Woods Hole Oceanographic Institution.

\begin{tabular}{|l|l|}
\hline Vehicle Property & Value \\
\hline length & $2.14 \mathrm{~m}(84 \mathrm{in})$. \\
mass & $1140 \mathrm{Kg}(2500 \mathrm{lbm})$ \\
Maximum Thrust, $x$ direction & $445 \mathrm{~N}(100 \mathrm{lbf})$ \\
Maximum Thrust, $y$ direction & $445 \mathrm{~N}(100 \mathrm{lbf})$ \\
Maximum Thrust-Generated Torque & $271 \mathrm{~N}-\mathrm{m}(200 \mathrm{ft}-\mathrm{lbf})$ \\
\hline
\end{tabular}

If we select $K_{F}=10, K_{m}=500$, and $K_{l}=10$ we find that the remaining scale factors must be $K_{t}=\sqrt{500}$ and $K_{\tau}=100$.

\section{A.4 Kinematics}

\section{A.4.1 Geometry of an open circuit cable drive}

The proposed mechanism consists of for open circuit cable drives which share a common output pulley. Therefore, to simplify the following kinematic derivations we first analyze the kinematics of an independent open circuit cable drive. 


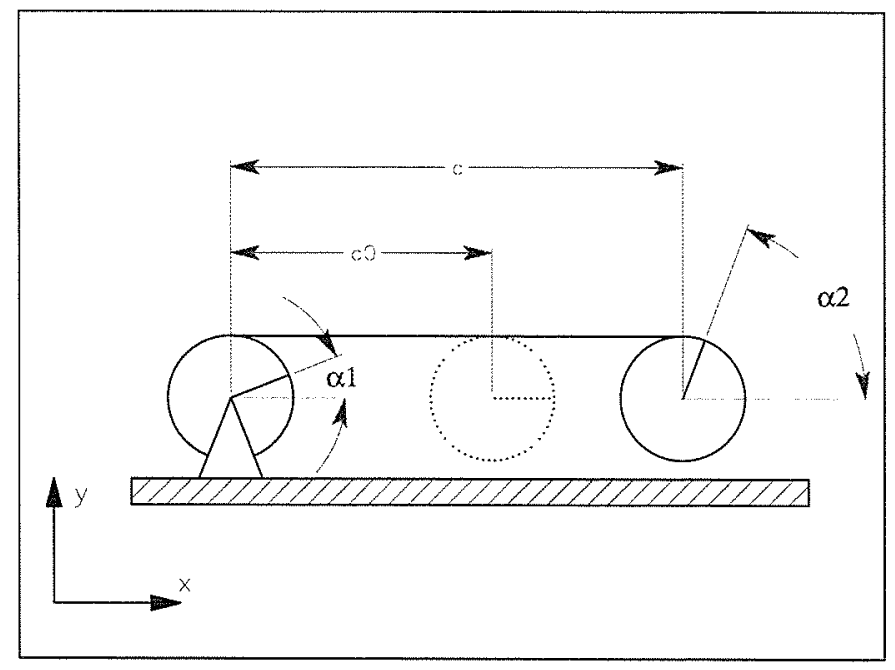

Figure A-2: Geometry of a cable connecting two pulleys of the same radius

We restrict our scope in this section to open circuit cable drives whose pulleys are both the same radius and whose cable runs from one pulley to the other without crossing a line drawn between the pulley axes, as shown in A-2. Such open circuit drives have a unique geometric property which permits the derivation of closed form solutions for the most of the results derived in this chapter. This property is that the length of the tangent portion of the cable always equals the distance between the pulley axes independent of distance $d$ separating the pulley axes (see A-2). In addition, the angle of the cable always equals the angle $\phi$ of the line connecting the pulley axes. This is not true for drives whose pulleys have different radii, and using such open circuit drives to construct our mechanism would require the use of numerical methods to evaluate the forward kinematics and Jacobian.

Figure A-2 shows two identical, rigid body pulleys linked by a laterally flexible but axially inextensible cable whose ends are rigidly affixed to the pulleys. Given the rigidity of all the elements the total length of the cable $s$ remains constant regardless of the relative positioning and rotation of the pulleys. Computing the total length $s$ when both pulley rotation angles $\alpha_{a}$ and $\alpha_{b}$ are equal to zero we get (assuming that 
the loads acting on the system are such that the cable never goes slack)

$$
s=\beta_{a_{0}} R+d_{0}+\beta_{b_{0}} R
$$

where $\beta_{a_{0}} R$ and $\beta_{b_{0}} R$ are the initial amounts of cable wrapped on pulleys $a$ and $b$ and $d_{0}$ is the initial separation between the pulley axes. When $\alpha_{a}$ and $\alpha_{b}$ are non-zero we obtain

$$
s=\left(\beta_{a_{0}}-\alpha_{a}\right) R+d+\left(\beta_{b_{0}}+\alpha_{b}\right) R
$$

Equating A.12 and A.13 and solving for the current separation $d$ as a function of the pulley rotation angles yields

$$
d=\left(\alpha_{a}-\alpha_{b}\right) R+d_{0}
$$

For a system in which the cable runs over, instead of under, the pulleys the equation for $d$ is

$$
d=-\left(\alpha_{a}-\alpha_{b}\right) R+d_{0}
$$

\section{A.4.2 Inverse Kinematics}

Using the results from the previous section we can model the geometry of the testbed. Referring to Figure A-3 we apply equation A.14 to model cables 1 and 3 and equation A.14 to model cables 2 and 4 we get 


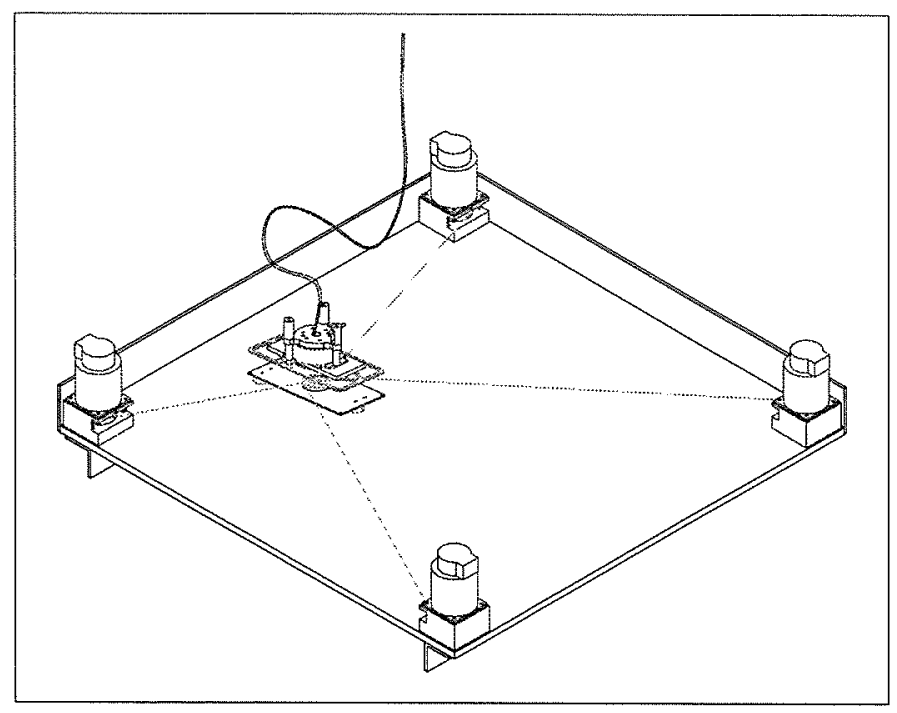

Figure A-3: Schematic of model vehicle cable drive mechanism

$$
\begin{aligned}
& d_{1}=\left(\alpha_{1}-\alpha_{5}\right) R+d 0_{1} \\
& d_{2}=-\left(\alpha_{2}-\alpha_{5}\right) R+d 0_{2} \\
& d_{3}=\left(\alpha_{3}-\alpha_{5}\right) R+d 0_{3} \\
& d_{4}=-\left(\alpha_{4}-\alpha_{5}\right) R+d 0_{4}
\end{aligned}
$$

From basic geometry we also have

$$
\begin{aligned}
& d_{1}^{2}=\left(x_{5}-x_{1}\right)^{2}+\left(y_{5}-y_{1}\right)^{2} \\
& d_{2}^{2}=\left(x_{5}-x_{2}\right)^{2}+\left(y_{5}-y_{2}\right)^{2} \\
& d_{3}^{2}=\left(x_{5}-x_{3}\right)^{2}+\left(y_{5}-y_{3}\right)^{2} \\
& d_{4}^{2}=\left(x_{5}-x_{4}\right)^{2}+\left(y_{5}-y_{4}\right)^{2}
\end{aligned}
$$

Solving the inverse kinematics problem for the table mechanism requires that 
we determine what motor angles $\alpha_{1}$ through $\alpha_{4}$ are required to place the vehicle at a given $\left[x_{5}, y_{5}, \alpha_{5}\right]$. For serial link mechanisms (e.g. most robot arms) solving the inverse kinematics problem typically proves to be quite difficultwhile solving the forward kinematics problem (i.e. knowing the motor angles, determine the position and orientation of the end effector) presents little challenge. In contrast, solving the inverse kinematics problem for the testbed (which is a parallel link mechanism) is actually easier to solve than the forward kinematics.

Given $\left[x_{5}, y_{5}, \alpha_{5}\right]$ we use A.20 through A.23 to solve for the $d_{i}$ and substitute into A.16 through A.19 to get

$$
\begin{aligned}
& \alpha_{1}=\frac{d_{1}-d 0_{1}}{R_{1}}+\alpha_{5} \\
& \alpha_{2}=\frac{d_{2}-d 0_{2}}{R_{2}}+\alpha_{5} \\
& \alpha_{3}=\frac{d_{3}-d 0_{3}}{R_{3}}+\alpha_{5} \\
& \alpha_{4}=\frac{d_{4}-d 0_{4}}{R_{4}}+\alpha_{5}
\end{aligned}
$$

where

$$
R_{i}=(\text { Rigging })_{i} R, \quad i=1, \ldots, 4
$$

where $(\text { Rigging })_{i}$ is +1 if cable $i$ gets tighter when pulley $i$ is rotated the positive (i.e. $\mathrm{CCW}$ ) direction and is -1 if such a rotation makes cable $i$ go slack.

\section{A.4.3 Forward Kinematics}

The forward kinematics are somewhat more difficult to obtain. We begin by substituting A.16 through A.19 into equations A.20 through A.23 and expanding to get 


$$
\begin{aligned}
& x_{5}^{2}-2 x_{5} x_{1}+x_{1}^{2}+y_{5}^{2}-2 y_{5} y_{1}+y_{1}^{2}=R^{2}\left(\alpha_{1}-\alpha_{5}\right)^{2}+2\left(\alpha_{1}-\alpha_{5}\right) R_{1} d 0_{1}+d 0_{1}^{2} \\
& x_{5}^{2}-2 x_{5} x_{2}+x_{2}^{2}+y_{5}^{2}-2 y_{5} y_{2}+y_{2}^{2}=R^{2}\left(\alpha_{2}-\alpha_{5}\right)^{2}+2\left(\alpha_{2}-\alpha_{5}\right) R_{2} d 0_{2}+d 0_{2}^{2} \\
& x_{5}^{2}-2 x_{5} x_{3}+x_{3}^{2}+y_{5}^{2}-2 y_{5} y_{3}+y_{3}^{2}=R^{2}\left(\alpha_{3}-\alpha_{5}\right)^{2}+2\left(\alpha_{3}-\alpha_{5}\right) R_{3} d 0_{3}+d 0_{3}^{2} \\
& x_{5}^{2}-2 x_{5} x_{4}+x_{4}^{2}+y_{5}^{2}-2 y_{5} y_{4}+y_{4}^{2}=R^{2}\left(\alpha_{4}-\alpha_{5}\right)^{2}+2\left(\alpha_{4}-\alpha_{5}\right) R_{4} d 0_{4}+d 0_{4}^{2}
\end{aligned}
$$

Subtracting A.29 from A.30 yields

$$
\begin{aligned}
& 2\left(x_{1}-x_{2}\right) x_{5}+2\left(y_{1}-y_{2}\right) y_{5}+x_{2}^{2}-x_{1}^{2}+y_{2}^{2}-y_{1}^{2}= \\
& R^{2}\left(2\left(\alpha_{1}-\alpha_{2}\right) \alpha_{5}+\alpha_{2}^{2}-\alpha_{1}^{2}\right)+2\left(\alpha_{2}-\alpha_{5}\right) R_{2} d 0_{2}-2\left(\alpha_{1}-\alpha_{5}\right) R_{1} d 0_{1}+d 0_{2}^{2}-d 0_{1}^{2}
\end{aligned}
$$

Likewise, subtracting A.31 from A.30 yields

$$
\begin{aligned}
& 2\left(x_{3}-x_{2}\right) x_{5}+2\left(y_{3}-y_{2}\right) y_{5}+x_{2}^{2}-x_{3}^{2}+y_{2}^{2}-y_{3}^{2}= \\
& R^{2}\left(2\left(\alpha_{3}-\alpha_{2}\right) \alpha_{5}+\alpha_{2}^{2}-\alpha_{3}^{2}\right)+2\left(\alpha_{2}-\alpha_{5}\right) R_{2} d 0_{2}-2\left(\alpha_{3}-\alpha_{5}\right) R_{3} d 0_{3}+d 0_{2}^{2}-d 0_{3}^{2}
\end{aligned}
$$

We may rewrite these as

$$
\begin{aligned}
& c_{1} x_{5}+c_{2} y_{5}+c_{3} \alpha_{5}=c_{4} \\
& c_{5} x_{5}+c_{6} y_{5}+c_{7} \alpha_{5}=c_{8}
\end{aligned}
$$

where

$$
\begin{aligned}
& c_{1}=2\left(x_{1}-x_{2}\right) \\
& c_{2}=2\left(y_{1}-y_{2}\right) \\
& c_{3}=2 R^{2}\left(\alpha_{2}-\alpha_{1}\right)+2 R_{2} d 0_{2}-2 R_{1} d 0_{1} \\
& c_{4}=x_{1}^{2}-x_{2}^{2}+y_{1}^{2}-y_{2}^{2}+R^{2}\left(\alpha_{2}^{2}-\alpha_{1}^{2}\right)
\end{aligned}
$$




$$
\begin{aligned}
& +2 \alpha_{2} R_{2} d 0_{2}-2 \alpha_{1} R_{1} d 0_{1}+d 0_{2}^{2}-d 0_{1}^{2} \\
c_{5}= & 2\left(x_{3}-x_{2}\right) \\
c_{6}= & 2\left(y_{3}-y_{2}\right) \\
c_{7}= & 2 R^{2}\left(\alpha_{2}-\alpha_{3}\right)+2 R_{2} d 0_{2}-2 R_{3} d 0_{3} \\
c_{8}= & x_{3}^{2}-x_{2}^{2}+y_{3}^{2}-y_{2}^{2}+R^{2}\left(\alpha_{2}^{2}-\alpha_{3}^{2}\right) \\
& +2 \alpha_{2} R_{2} d 0_{2}-2 \alpha_{3} R_{3} d 0_{3}+d 0_{2}^{2}-d 0_{3}^{2}
\end{aligned}
$$

Solving A.35 and A.36 for $x_{5}$ and $y_{5}$ in terms of $\alpha_{5}$ yields

$$
\begin{aligned}
& x_{5}=k_{1} \alpha_{5}+k_{2} \\
& y_{5}=k_{3} \alpha_{5}+k_{4}
\end{aligned}
$$

where

$$
\begin{aligned}
& k_{1}=\frac{c_{6} c_{3}-c_{2} c_{7}}{c_{5} c_{2}-c_{6} c_{1}} \\
& k_{2}=\frac{c_{8} c_{2}-c_{6} c_{4}}{c_{5} c_{2}-c_{6} c_{1}} \\
& k_{3}=-\frac{c_{5} k_{1}+c_{7}}{c_{6}} \\
& k_{4}=\frac{c_{8}-c_{5} k_{2}}{c_{6}}
\end{aligned}
$$

Substituting for $x_{5}$ and $y_{5}$ in A.30 we get

$$
A \alpha_{5}^{2}+B \alpha_{5}+C=0
$$


where

$$
\begin{aligned}
A & =k_{1}^{2}+k_{3}^{2}-R^{2} \\
B & =2\left(k_{2}-x_{2}\right) k_{1}+2\left(k_{4}-y_{2}\right) k_{3}+2 \alpha_{2} R^{2}+2 R_{2} d 0_{2} \\
C & =x_{2}^{2}+y_{2}^{2}+k_{2}^{2}+k_{4}^{2}-2 k_{2} x_{2}-2 k_{4} y_{2}-\alpha_{2}^{2} R^{2}-2 \alpha_{2} R_{2} d 0_{2}-d 0_{2}^{2}
\end{aligned}
$$

Solving for $\alpha_{5}$ we obtain

$$
\alpha_{5}=\frac{-B \pm \sqrt{B^{2}-4 A C}}{2 A}
$$

To complete the forward kinematics solution we substitute $\alpha_{5}$ into A.46 and A.47 to obtain $x_{5}$ and $y_{5}$.

\section{A.4.4 The Jacobian}

The Jacobian for the system relates the vector of vehicle velocities to the vector of motor velocities, i.e.

$$
\left[\dot{x}_{5}, \dot{y}_{5}, \dot{\alpha}_{5}\right]^{T}=J\left[\dot{\alpha}_{1}, \dot{\alpha}_{2}, \dot{\alpha}_{3}\right]^{T}
$$

To find $\dot{x}_{5}, \dot{y}_{5}$ and $\dot{\alpha}_{5}$ we differentiate A.46, A.47 and A.56, respectively, with respect to time. Doing so we obtain

$$
\begin{aligned}
\dot{x}_{5} & =\alpha_{5} \dot{k}_{1}+k_{1} \dot{\alpha}_{5}+\dot{k}_{2} \\
\dot{y}_{5} & =\alpha_{5} \dot{k}_{3}+k_{3} \dot{\alpha}_{5}+\dot{k}_{4} \\
\dot{\alpha}_{5} & =\frac{-\dot{B} \pm \frac{B \dot{B}-2 C \dot{A}-2 A \dot{C}}{\sqrt{B^{2}-4 A C}}}{2 A}-\frac{-B \pm \sqrt{B^{2}-4 A C}}{2 A^{2}} \dot{A} \\
& =\frac{-\dot{B} \pm \frac{B \dot{B}-2 C \dot{A}-2 A \dot{C}}{\sqrt{B^{2}-4 A C}}}{2 A}-\alpha_{5} \frac{\dot{A}}{A}
\end{aligned}
$$


Differentiating A.53 through A.55 yields the unknown $\dot{A}, \dot{B}$ and $\dot{C}$

$$
\begin{aligned}
\dot{A} & =2\left(k_{1} \dot{k}_{1}+k_{3} \dot{k}_{3}\right) \\
\dot{B} & =2\left(k_{1} \dot{k}_{2}+k_{2} \dot{k}_{1}\right)+2\left(k_{3} \dot{k}_{4}+k_{4} \dot{k}_{3}\right)+2 R^{2} \dot{\alpha}_{2} \\
\dot{C} & =2\left(k_{2} \dot{k}_{2}+k_{4} \dot{k}_{4}\right)-2 x_{2} \dot{k}_{2}-2 y_{2} \dot{k}_{4}-2 R^{2} \alpha_{2} \dot{\alpha}_{2}-2 R_{2} d 0_{2} \dot{\alpha}_{2}
\end{aligned}
$$

where, by differentiating A.48 through A.51 we have

$$
\begin{aligned}
& \dot{k}_{1}=\frac{c_{6} \dot{c}_{3}-c_{2} \dot{c}_{7}}{c_{5} c_{2}-c_{6} c_{1}} \\
& \dot{k}_{2}=\frac{c_{2} \dot{c}_{8}-c_{6} \dot{c}_{4}}{c_{5} c_{2}-c_{6} c_{1}} \\
& \dot{k}_{3}=\frac{\dot{c}_{7}-c_{5} \dot{k}_{1}}{c_{6}} \\
& \dot{k}_{4}=\frac{\dot{c}_{8}-c_{5} \dot{k}_{2}}{c_{6}}
\end{aligned}
$$

Finally, we determine the unkown $\dot{c}_{i}$ by differentiating A.37 through A.44 which yields

$$
\begin{aligned}
& \dot{c_{1}}=0 \\
& \dot{c_{2}}=0 \\
& \dot{c_{3}}=2 * R^{2}\left(\dot{\alpha}_{2}-\dot{\alpha}_{1}\right) \\
& \dot{c_{4}}=2 * R^{2}\left(\alpha_{2} \dot{\alpha}_{2}-\alpha_{1} \dot{\alpha}_{1}\right)+2 R_{2} d 0_{2} \dot{\alpha}_{2}-2 R_{1} d 0_{1} \dot{\alpha}_{1} \\
& \dot{c_{5}}=0 \\
& \dot{c_{6}}=0 \\
& \dot{c_{7}}=2 * R^{2}\left(\dot{\alpha}_{2}-\dot{\alpha}_{3}\right)
\end{aligned}
$$




$$
\dot{c_{8}}=2 * R^{2}\left(\alpha_{2} \dot{\alpha}_{2}-\alpha_{3} \dot{\alpha}_{3}\right)+2 R_{2} d 0_{2} \dot{\alpha}_{2}-2 R_{3} d 0_{3} \dot{\alpha}_{3}
$$

\section{A.5 Statics}

\section{A.5.1 Ensuring Positive Cable Tensions}

Cables cannot transmit compressive loads. We must ensure that the cables are always in tension. To do this we use four actuators to control the vehicle's three degrees of freedom. The presence of the fourth actuator allows gives us some control over the internal tension of the system. The approach we use is that used by Salisbury to ensure positive tensioning of the four actuator cables used to controleach three degree of freedom finger in the JPL/Stanford cable driven hand.

The matrix relating the motor torques to the force/moment vector applied to the vehicle is a 3 by 4 matrix of rank 3 . The null space of this matrix is a vector of motor torques which result in zero net force/moment applied to the vehicle. To guarantee positive tensions we use the three by three Jacobian derived above to determine three of the motor torques. We use the smallest (i.e. most negative) of these torques to determine what multiple of the null space vector must be added to the motor torque vector to ensure that the cable tensions are positive.

$$
\begin{aligned}
f & =J^{-T} \tau \\
J^{-T} & =\frac{1}{R}\left[\begin{array}{rrrr}
\cos \phi_{1} & -\cos \phi_{2} & \cos \phi_{3} & -\cos \phi_{4} \\
\sin \phi_{1} & -\sin \phi_{2} & \sin \phi_{3} & -\sin \phi_{4} \\
R & R & R & R
\end{array}\right]
\end{aligned}
$$




$$
=\left[\begin{array}{rrrr}
a_{1} & a_{2} & a_{3} & a_{4} \\
b_{1} & b_{2} & b_{3} & b_{4} \\
1 & 1 & 1 & 1
\end{array}\right]
$$

Null Space of $J^{-T}$

$$
J^{-T} E=0
$$

$$
\begin{aligned}
& E_{1}=1 \\
& E_{2}=\frac{K_{1}-K_{2}}{K_{3}-K_{4}} \\
& E_{3}=K_{1}+E_{2} K_{4} \\
& E_{4}=-1+2 E_{2}
\end{aligned}
$$

where

$$
\begin{aligned}
& K_{1}=\frac{b_{1}-b_{4}}{b_{4}-b_{3}} \\
& K_{2}=\frac{a_{1} b_{4}-a_{4} b_{1}}{a_{4} b_{3}-a_{3} b_{4}} \\
& K_{3}=\frac{a_{2} b_{4}-a_{4} b_{2}}{a_{4} b_{3}-a_{3} b_{4}} \\
& K_{4}=\frac{b_{2}-b_{4}}{b_{4}-b_{3}}
\end{aligned}
$$

\section{A.6 Transmission Non-idealities}

The derivations in the kinematics, statics, and dynamics sections assume that the transmission (i.e. the components connecting the actuators to the surrogate vehi- 
cle) undergo no deformation when subjected to transmitted loads. In this section we model the deformation characteristics of the transmission in order to ensure the validity of this approximation.

\section{A.6.1 Stiffness of Open Circuit Cable Drive}

As discussed in the kinematics section, our mechanism consists of four open circuit cable drives connected in parallel to the surrogate vehicle. Thus we begin our analysis by considering the load/deformation characteristics of a single open circuit drive. Using this result, we can construct a stiffness model for the aggregate system.

Consider the uniformly pretensioned system shown in figure ?? ( by uniformly pretensioned we mean that the cable has been wrapped such that its tension is initially everywhere equal to some value (note to me: this figure should have the cable running tangent from the BOTTOM of the left hand pulley (pulley 1) to the BOTTOM of the right hand pulley ( pulley 2))

$$
T=F+\frac{M}{r_{2}}
$$

To find the total increase in length of the cable we sum the elongations of the cable in the tangent length and the cable wrapped on each pulley. The load/deformation characteristics of this system was studied in detail in [27] and the total cable elongation was shown to be

$$
\delta=\frac{r_{2}}{E A \mu_{e f f}^{*}}\left[(G F+1)\left(\frac{T}{T_{0}}-1\right)-\log \frac{T}{T_{0}}\right]
$$

where $E$ is the cable material's modulus of elasticity, $A$ is the cable's effective cross sectional area, and $G F$ and $\mu_{e f f}^{*}$ the geometry friction number and the effective coefficient of friction respectively, defined as 


$$
\begin{aligned}
G F & =\frac{L \mu_{e f f}^{*}}{r_{2}} \\
\mu_{e f f}^{*} & =\left(\frac{1}{\mu_{r_{1}}}+\frac{r_{2}}{r_{1}} \frac{1}{\mu_{r_{2}}}\right)^{-1} \operatorname{sign}\left(\frac{T}{T_{0}}-1\right)
\end{aligned}
$$

where $L$ is the length of the tangent portion of the cable and $\mu_{r_{1}}$ and $\mu_{r_{2}}$ are the coefficients of dynamic friction between the cable and pulleys 1 and 2 respectively. For our system $r_{1}=r_{2}=R$ which means that $L=d$ and, assuming that $\mu_{r_{1}}=\mu_{r_{2}}=\mu$, also yields $\mu_{e f f}^{*}=\frac{\mu}{2} \operatorname{sign}\left(\frac{T}{T_{0}}-1\right)$.

To find the stiffness we take the derivative of $\delta$ with respect to $T$ to get $\frac{d \delta}{d T}$ and then invert this to get $k=\frac{d T}{d \delta}$, yielding

$$
k=\frac{E A \mu_{e f f}^{*}}{R\left(G F+1-\frac{T_{0}}{T}\right)}
$$

Unfortunately the cable has a nonlinear stiffness which, as shown in [27], depends inversely on the tension in the tangent length of cable. However, we can identify two assymptotic stiffness values:

$$
\begin{aligned}
& k_{T \ll T_{0}}=0 \\
& k_{T \gg T_{0}}=\frac{E A \mu_{\text {eff }}^{*}}{(G F+1) R}
\end{aligned}
$$

Presuming successful implementation of the technique described in the statics section, the cable tensions will never drop below some minimum value which we can substitute for $T_{0}$. Thus using A.96 provides a conservative estimate for the cable stiffness and greatly simplifies further analysis by enabling us to model the cable as a linear spring element. 


\section{A.6.2 Resonant Frequencies}

Having a model for the cable deformation allows us to model the load/deformation behavior of complete system. Assuming that the only significant compliance is that contributed by the cables, the mechanism has 7 degrees of freedom (D.O.F.): one rotational D.O.F. for each motor pulley and two translational and one rotational D.O.F. for the surrogate vehicle.

\section{Equilibrium equations}

In the absence of any externally applied loads the dynamic equilibrium equations for the mechanism are

$$
\begin{aligned}
& J_{1} \ddot{\alpha}_{1}=F_{1} R \\
& J_{2} \ddot{\alpha}_{2}=-F_{2} R \\
& J_{3} \ddot{\alpha}_{3}=F_{3} R \\
& J_{4} \ddot{\alpha}_{4}=-F_{4} R \\
& J_{5} \ddot{\alpha}_{5}=\left(-F_{1}+F_{2}-F_{3}+F_{4}\right) R \\
& m_{5} \ddot{x}_{5}=-\sum F_{i} \cos \phi_{i} \\
& m_{5} \ddot{y}_{5}=-\sum F_{i} \sin \phi_{i}
\end{aligned}
$$

\section{Constitutive equations}

The constitutive equations relate the deformations of the cables to the forces experienced by the cables. To find these we apply A.96 to each of the cables which yields

$$
F_{i}=k_{i} \Delta s_{i}
$$


where $i=1 \ldots 4, \Delta s_{i}$ is the increase in length of cable $i$ and

$$
k_{i}=\frac{E A}{d_{i}+\frac{R}{\mu_{e f f_{i}}^{*}}}
$$

\section{Geometry of deformation}

To make use of the constitutive equations A.104 we need to solve for the $\Delta s_{i}$. Assume that the system is initially at a known configuration $\left(x_{5_{0}}, y_{5_{0}}, \alpha_{5_{0}}\right)$ and $\left(\alpha_{1_{0}}, \alpha_{2_{0}}, \alpha_{3_{0}}, \alpha_{4_{0}}\right)$. For this configuration we have

$$
\begin{aligned}
d_{i_{0}} & =\sqrt{\left(x_{5_{0}}-x_{i_{0}}\right)^{2}+\left(y_{5_{0}}-y_{i_{0}}\right)^{2}} \\
\phi_{i_{0}} & =\arctan \left(\frac{y_{5_{0}}-y_{i_{0}}}{x_{5_{0}}-x_{i_{0}}}\right)
\end{aligned}
$$

where $i=1 \ldots 4$ and $\left(x_{i}, y_{i}\right)$ are the $\mathrm{x}$ and $\mathrm{y}$ coordinates of the motor pulley axes. Geometrically, the length $s_{i}$ of cable $i$ at any given time equals the total path from its attachment point on motor pulley $i$ to attachment point of its other end on the the surrogate vehicle pulley (pulley 5), i.e.

$$
s_{i}=\beta_{i i} R+d_{i}+\beta_{i 5} R
$$

where $\beta_{i i} R$ is the length of cable $i$ presently wrapped on pulley $i$ and $\beta_{i 5} R$ is the length of cable $i$ wrapped on pulley 5. Recognizing that the rotation angle $\alpha_{i}$ of pulley $i$ increases or decreases the amount of wrapped cable on the pulley we can rewrite A.108 for each of the cables, yielding

$$
s_{1}=\left(\beta_{11_{0}}-\alpha_{1}\right) R+d_{1}+\left(\beta_{15_{0}}+\alpha_{5}\right) R
$$




$$
\begin{aligned}
& s_{2}=\left(\beta_{22_{0}}+\alpha_{2}\right) R+d_{2}+\left(\beta_{25_{0}}-\alpha_{5}\right) R \\
& s_{3}=\left(\beta_{33_{0}}-\alpha_{3}\right) R+d_{3}+\left(\beta_{35_{0}}+\alpha_{5}\right) R \\
& s_{4}=\left(\beta_{44_{0}}+\alpha_{4}\right) R+d_{4}+\left(\beta_{45_{0}}-\alpha_{5}\right) R
\end{aligned}
$$

To solve for the change in length $\Delta s_{i}$ of cable $i$ we subtract the initial value of $s_{i_{0}}$ from the present length $s_{i}$. The nonlinear dependence of the $d_{i}$ terms on the surrogate vehicle coordinates $\left(x_{5}, y_{5}\right)$ yields, when substituted back into the equilibrium equations, governing equations which have no closed form solution.

To avoid this problem we can restrict our analysis to small motions of the surrogate vehicle about its initial position $\left(x_{5}, y_{5}\right)$. Doing so, we may approximate the $\Delta s_{i}$ by linearizing the $s_{i}$ about a given $\left(x_{5}, y_{5}\right)$. The result is

$$
\begin{aligned}
\Delta s_{i} & =s_{i}-s_{i_{0}} \\
& \approx \frac{\delta s_{i}}{\delta x_{5}}\left(\Delta x_{5}\right)+\frac{\delta s_{i}}{\delta y_{5}}\left(\Delta y_{5}\right)+\frac{\delta s_{i}}{\delta \alpha_{5}}\left(\Delta \alpha_{5}\right)+\frac{\delta s_{i}}{\delta \alpha_{i}}\left(\Delta \alpha_{i}\right) \\
& \approx \frac{x_{5}-x_{i}}{\sqrt{d_{i}}} \Delta x+\frac{y_{5}-y_{i}}{\sqrt{d_{i}}} \Delta y \pm R\left(\Delta \alpha_{5}-\Delta \alpha_{i}\right) \\
& \approx \cos \phi_{i} \Delta x+\sin \phi_{i} \Delta y \pm R\left(\Delta \alpha_{5}-\Delta \alpha_{i}\right)
\end{aligned}
$$

where the appropriate choice of \pm depends particular cable being considered and

$$
\begin{aligned}
\Delta x_{5} & =x_{5}-x_{5_{0}} \\
\Delta y_{5} & =y_{5}-y_{5_{0}} \\
\Delta \alpha_{5} & =\alpha_{5}-\alpha_{5_{0}} \\
\Delta \alpha_{i} & =\alpha_{i}-\alpha_{i_{0}}
\end{aligned}
$$




\section{Equations of motion}

By substituting the $\Delta s_{i}$ 's and $k_{i}$ 's we can rewrite the equilibrium equations in matrix form as

$$
M \ddot{v}+K v=0
$$

where $v=\left[\alpha_{1}, \alpha_{2}, \alpha_{3}, \alpha_{4}, \alpha_{5}, x_{5}, y_{5}\right]^{T}$. The system mass matrix $M$ is given by

$$
M=\left[\begin{array}{rrrrrrr}
J_{1} & 0 & 0 & 0 & 0 & 0 & 0 \\
0 & J_{2} & 0 & 0 & 0 & 0 & 0 \\
0 & 0 & J_{3} & 0 & 0 & 0 & 0 \\
0 & 0 & 0 & J_{4} & 0 & 0 & 0 \\
0 & 0 & 0 & 0 & J_{5} & 0 & 0 \\
0 & 0 & 0 & 0 & 0 & m_{5} & 0 \\
0 & 0 & 0 & 0 & 0 & 0 & m_{5}
\end{array}\right]
$$

while $K$, the system stiffness matrix, equals

$$
\begin{aligned}
& K= \\
& {\left[\begin{array}{rrrrrrr}
-k_{1} R^{2} & 0 & 0 & 0 & k_{1} R^{2} & k_{1} R_{1} c_{1} & k_{1} R_{1} s_{1} \\
0 & -k_{2} R^{2} & 0 & 0 & k_{2} R^{2} & k_{2} R_{2} c_{2} & k_{2} R_{2} s_{2} \\
0 & 0 & -k_{3} R^{2} & 0 & k_{3} R^{2} & k_{3} R_{3} c_{3} & k_{3} R_{3} s_{3} \\
0 & 0 & 0 & -k_{4} R^{2} & k_{4} R^{2} & k_{4} R_{4} c_{4} & k_{4} R_{4} s_{4} \\
k_{1} R^{2} & k_{2} R^{2} & k_{3} R^{2} & k_{4} R^{2} & R^{2} \sum k_{i} & \sum k_{i} R_{i} c_{i} & \sum k_{i} R_{i} s_{i} \\
k_{1} R_{1} c_{1} & k_{2} R_{2} c_{2} & k_{3} R_{3} c_{3} & k_{4} R_{4} c_{4} & \sum k_{i} R_{i} c_{i} & \sum k_{i} c_{i}^{2} & \sum k_{i} s_{i} c_{i} \\
k_{1} R_{1} s_{1} & k_{2} R_{2} s_{2} & k_{3} R_{3} s_{3} & k_{4} R_{4} s_{4} & \sum k_{i} R_{i} s_{i} & \sum k_{i} s_{i} c_{i} & \sum k_{i} s_{i}^{2}
\end{array}\right]}
\end{aligned}
$$


where the summations are performed for $i=1 \ldots 4, c_{i}=\cos \phi_{i}, s_{i}=\sin \phi_{i}$, and, for our system,

$$
\begin{aligned}
& R_{1}=-R \\
& R_{2}=R \\
& R_{3}=-R \\
& R_{4}=R
\end{aligned}
$$

\section{A.6.3 Strum Frequencies}

In addition to the modes discussed above a cable under tension can also experience tranverse vibrations. For a cable of supported at both ends, having length $L$ and mass per unit length $\rho$ and subject to tension $T$ the fundamental tranverse vibrational frequencies are well known and are equal to

$$
f=\frac{n}{2} \sqrt{\frac{T}{\rho L^{2}}}
$$

Representative worst case values for the cables used to control the surrogate vehicle are $L=50 \mathrm{in}$., $\rho=3.3 e^{-4} \mathrm{lbm} . / \mathrm{in}$. and $T=.25 \mathrm{lbf}$. the fundamental frequency is 5 $\mathrm{Hz}$. 
Appendix B

Case 1: One Point Contact with

No Slip ( Pure Rotation) 


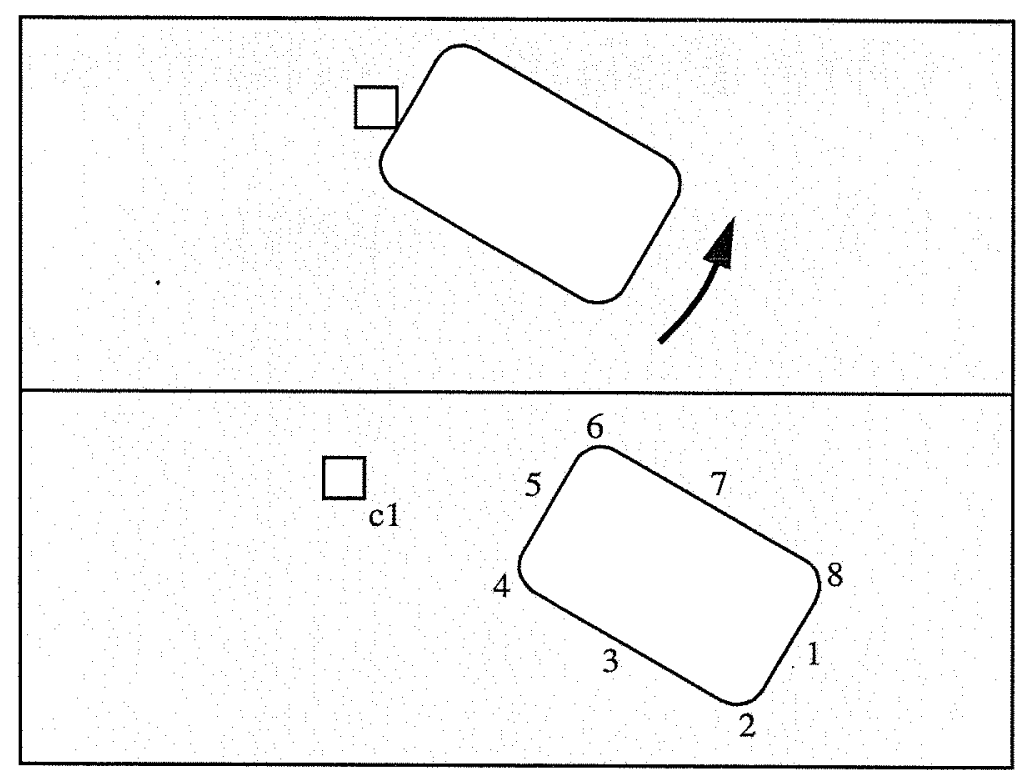

Figure B-1: Case 1 Experiment: Edge 5 of the vehicle was brought into contact with the corner $\mathrm{c} 1$ of a fixed object in environment. The vehicle was then rotated about this corner such that little or no slip occurred at the contact point. Initial rotation was the counter clockwise direction, then the rotation was reveresed, bringing the vehicle (approximately) back to its original position. The obstacle was a square bar of 6061 aluminum alloy. 


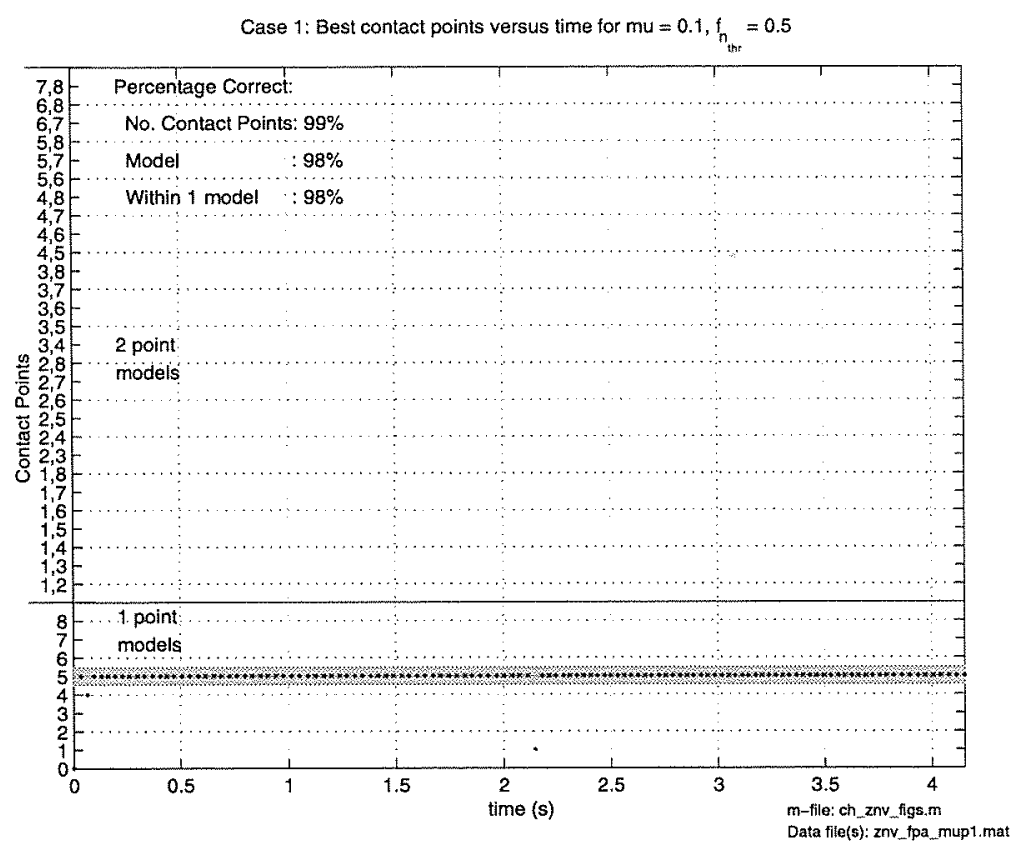

Figure B-2: Case 1: Best Overall Model vs. Time
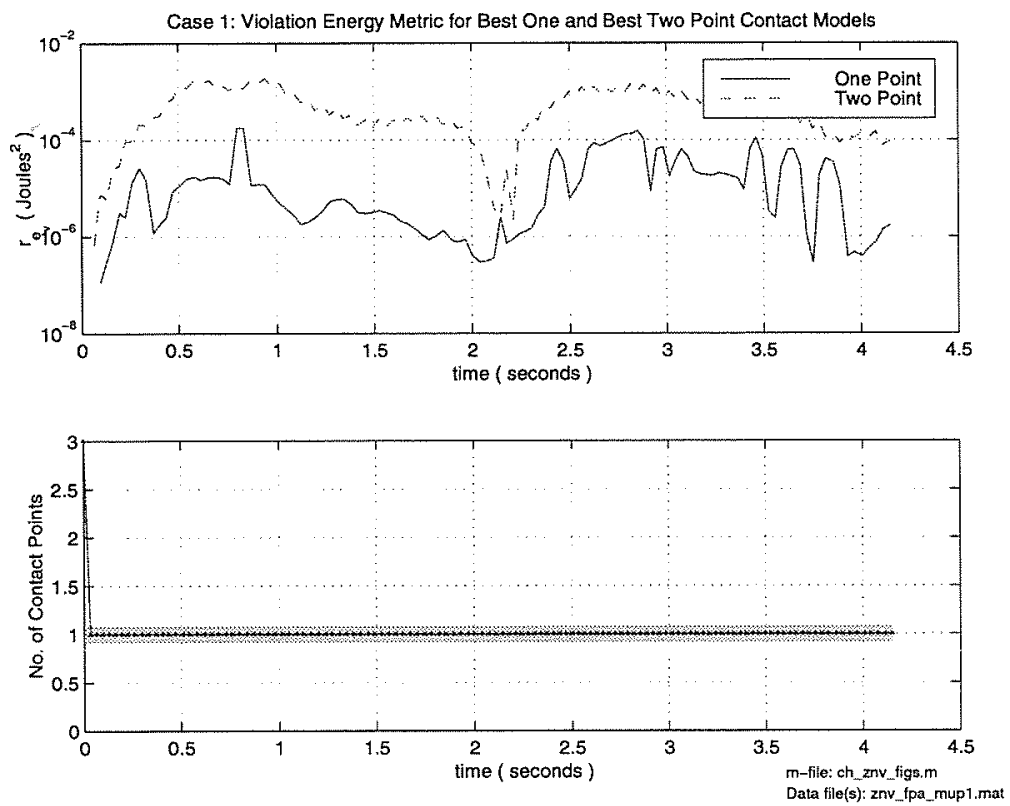

Figure B-3: Case 1: Best Number of Contact Points vs. Time 

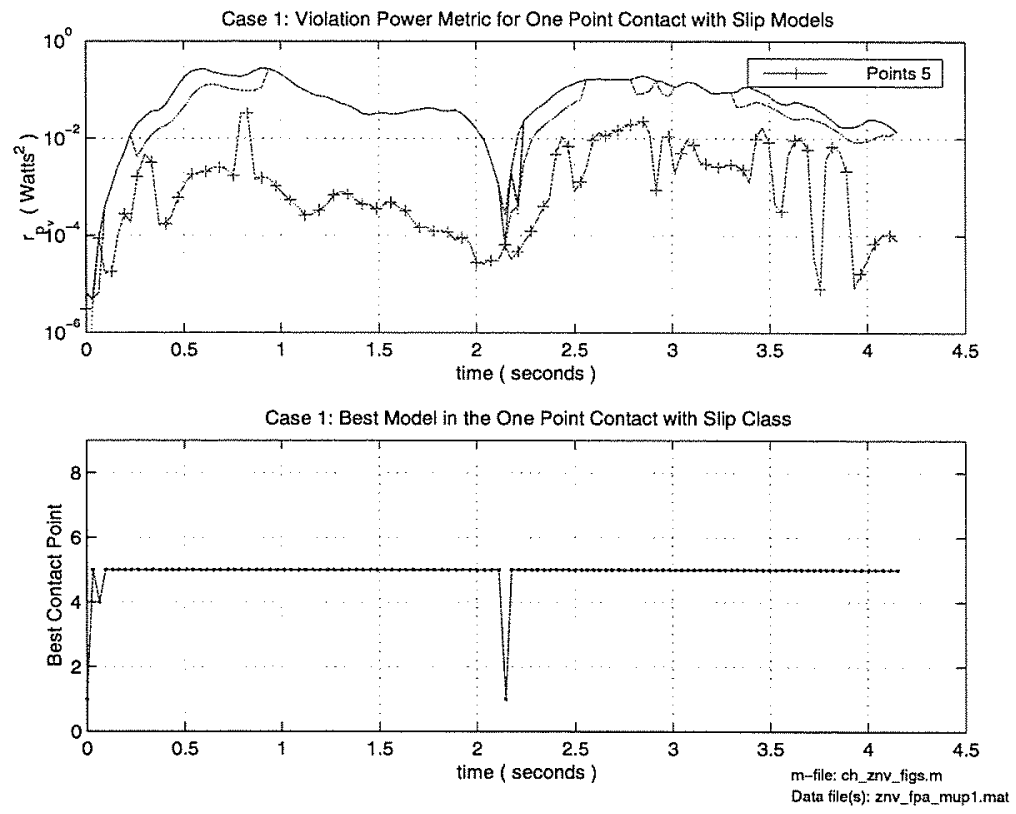

Figure B-4: Case 1: Best One Point Model vs. Time.
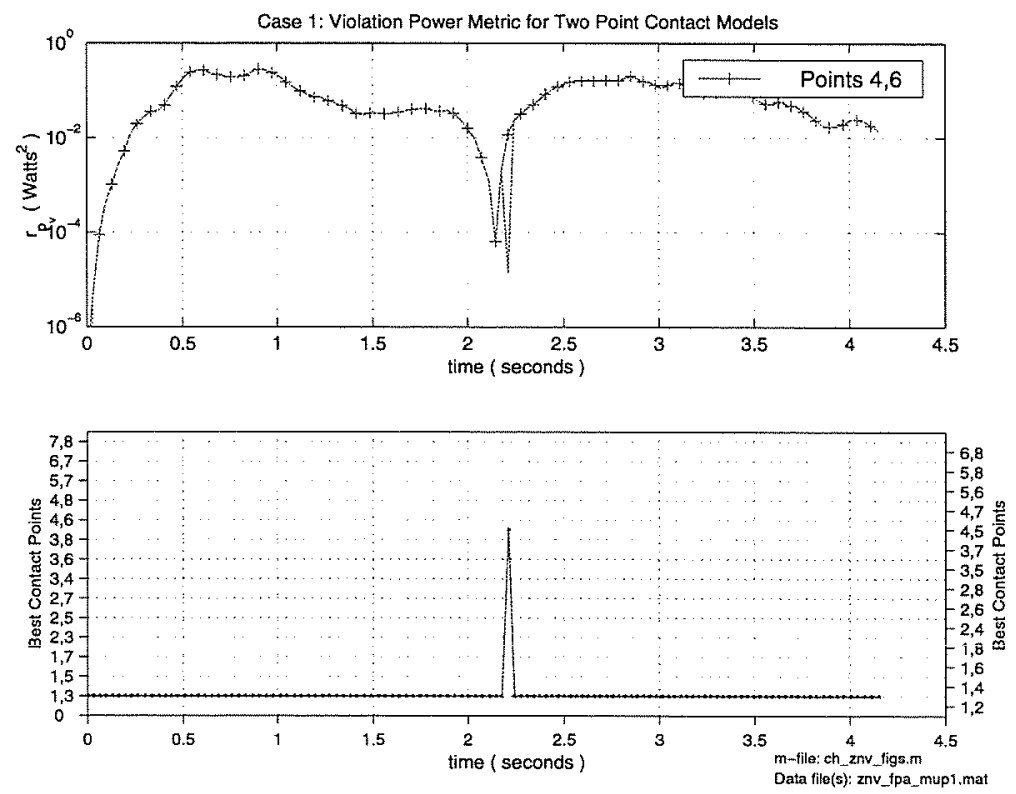

Figure B-5: Case 1: Best Two Point Model vs. Time. 

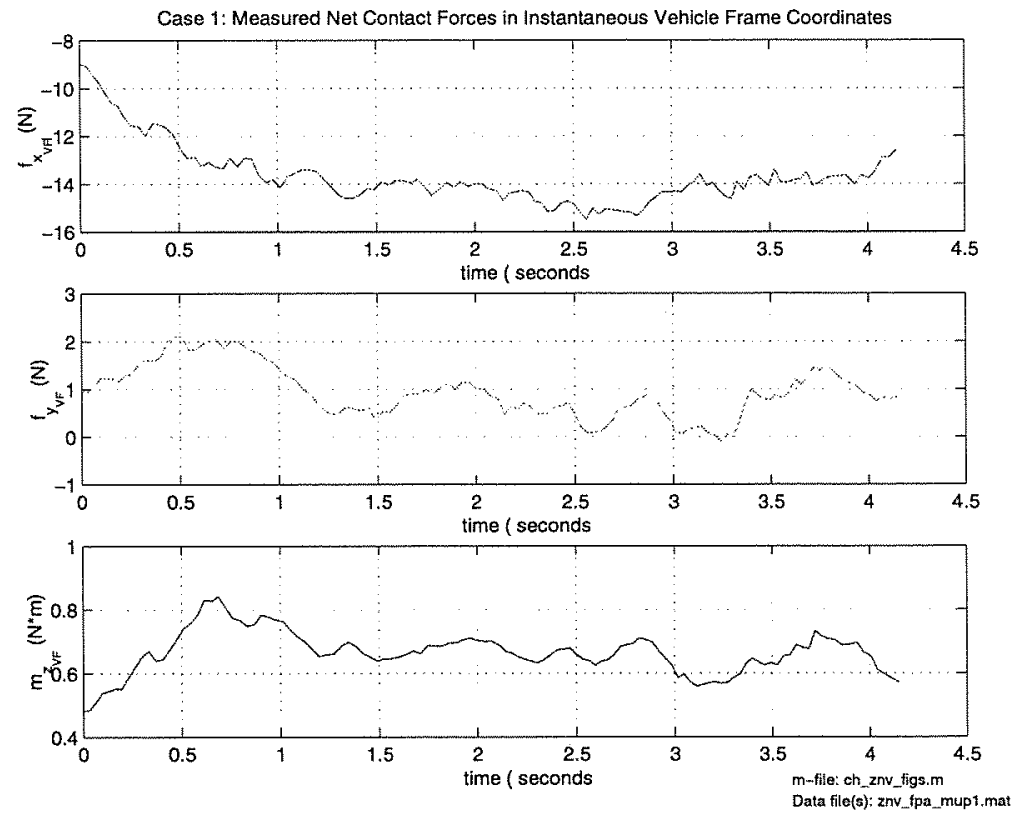

Figure B-6: Case 1: Measured Force and Moment vs. Time.
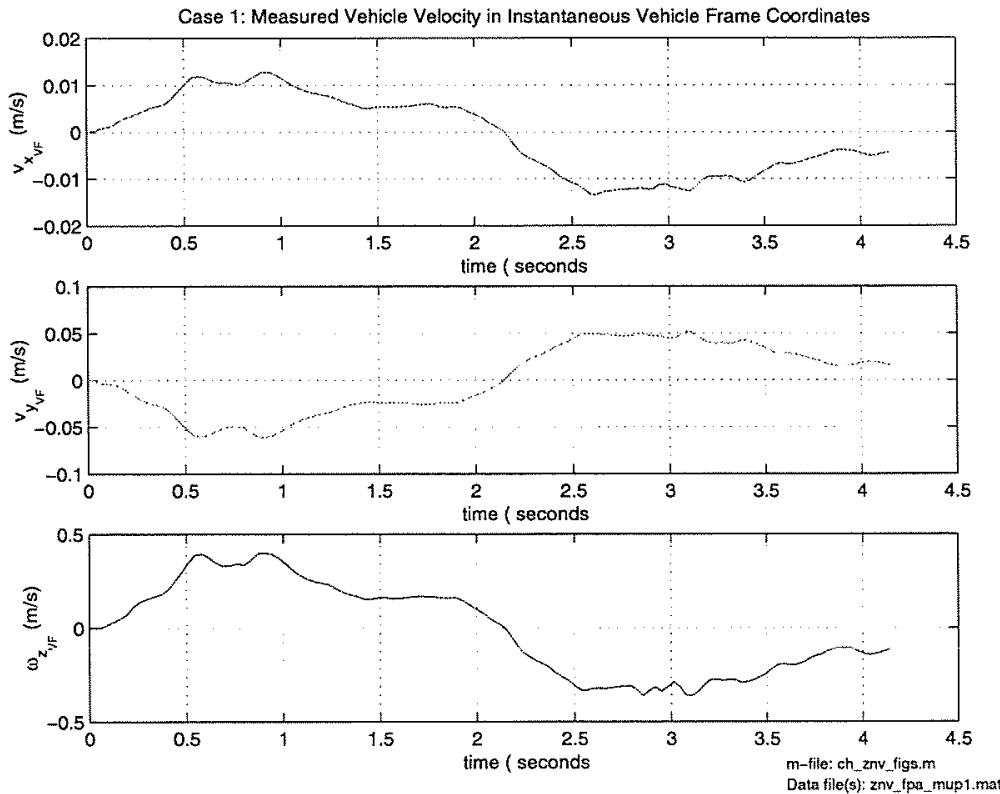

Figure B-7: Case 1: Measured Velocity vs. Time. 


\section{Appendix $\mathrm{C}$}

Case 2: One Point Contact with

Slip ( Rotation and Translation ) 


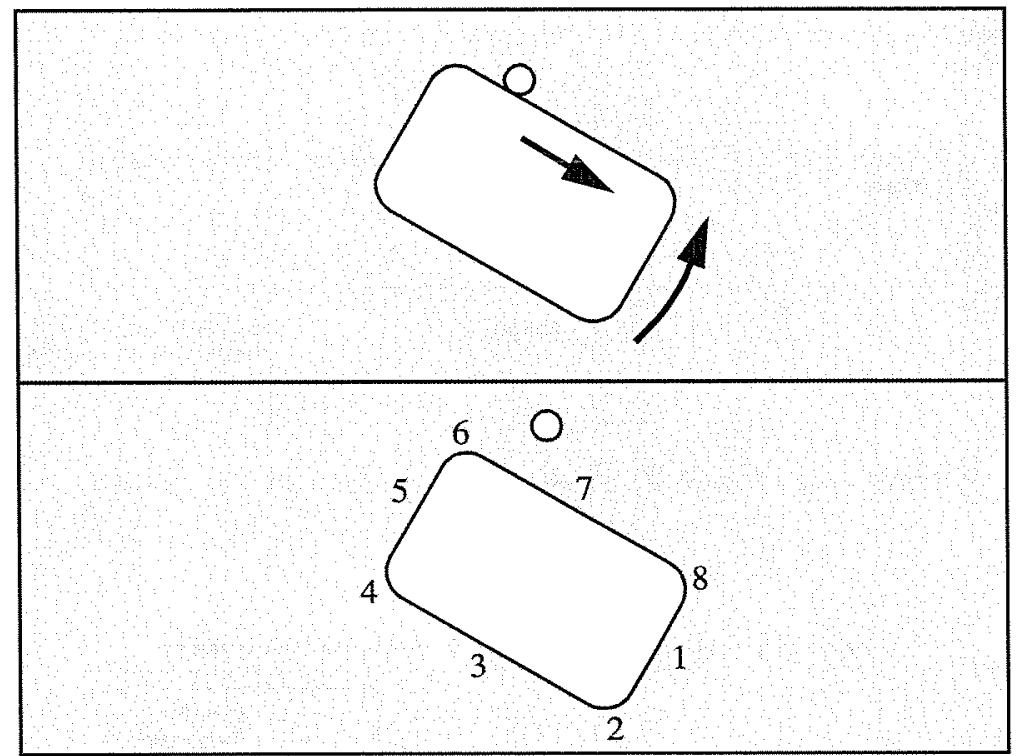

Figure C-1: Case 2 Experiment: Edge 5 of the vehicle was brought into contact with a fixed cylindrical obstacle. The vehicle was then moved such that it was in sliding contact, where the vehicle motion combined both rotation and translation of the vehicle relative to the contact point. The obstacle was a $12.7 \mathrm{~mm}$ diameter stainless steel bar. 


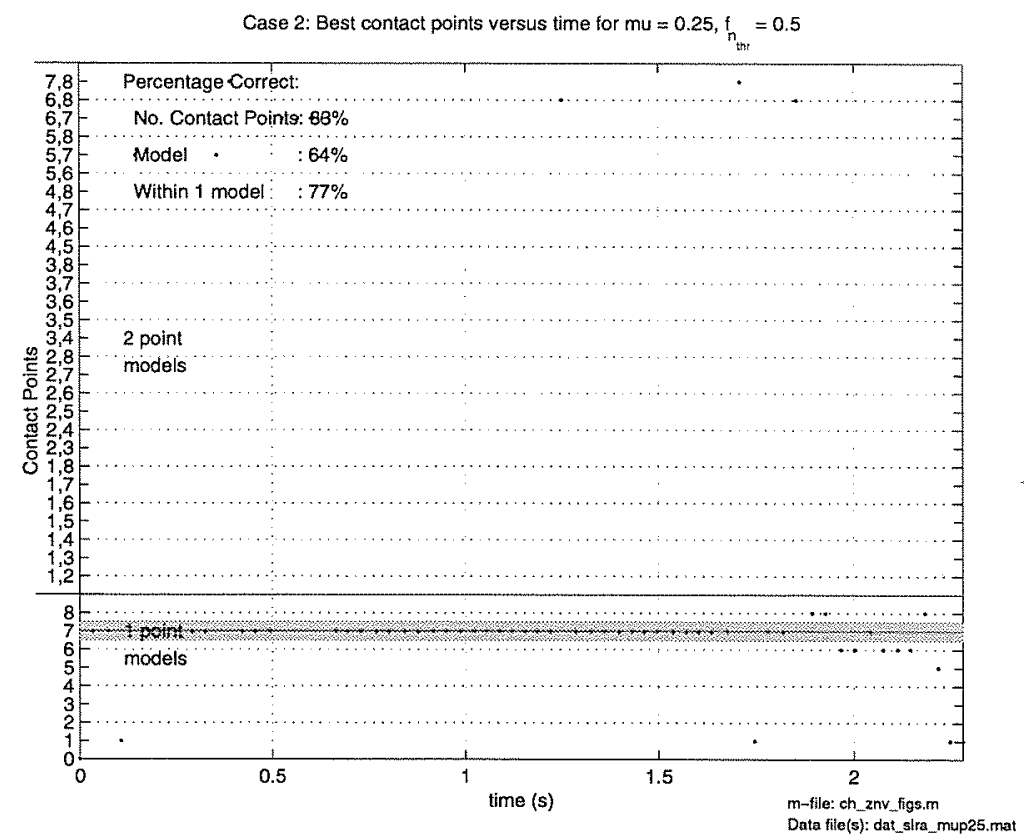

Figure C-2: Case 2: Best Overall Model vs. Time
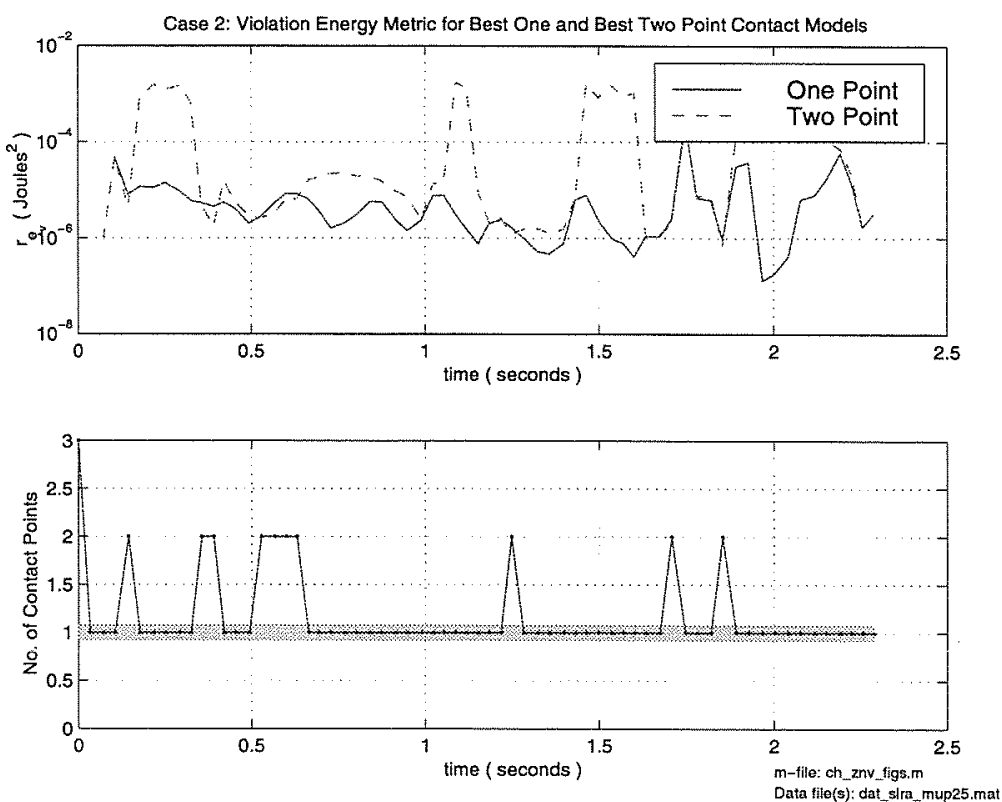

Figure C-3: Case: Best Number of Contact Points vs. Time 

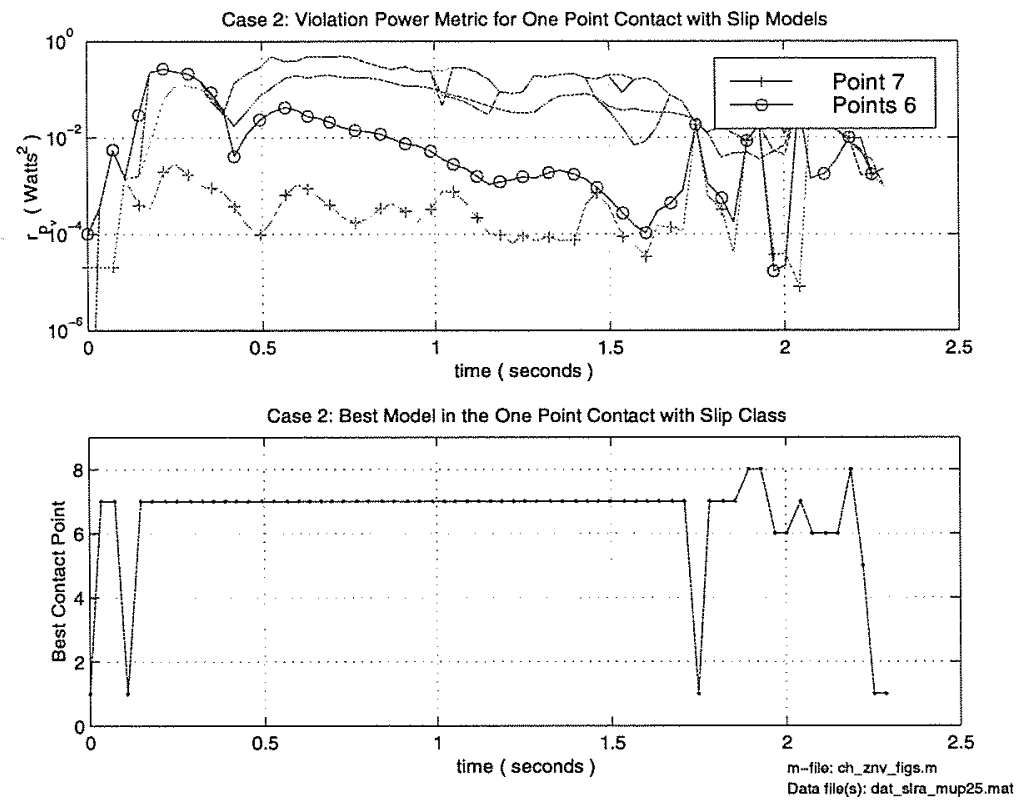

Figure C-4: Case 2: Best One Point Model vs. Time.
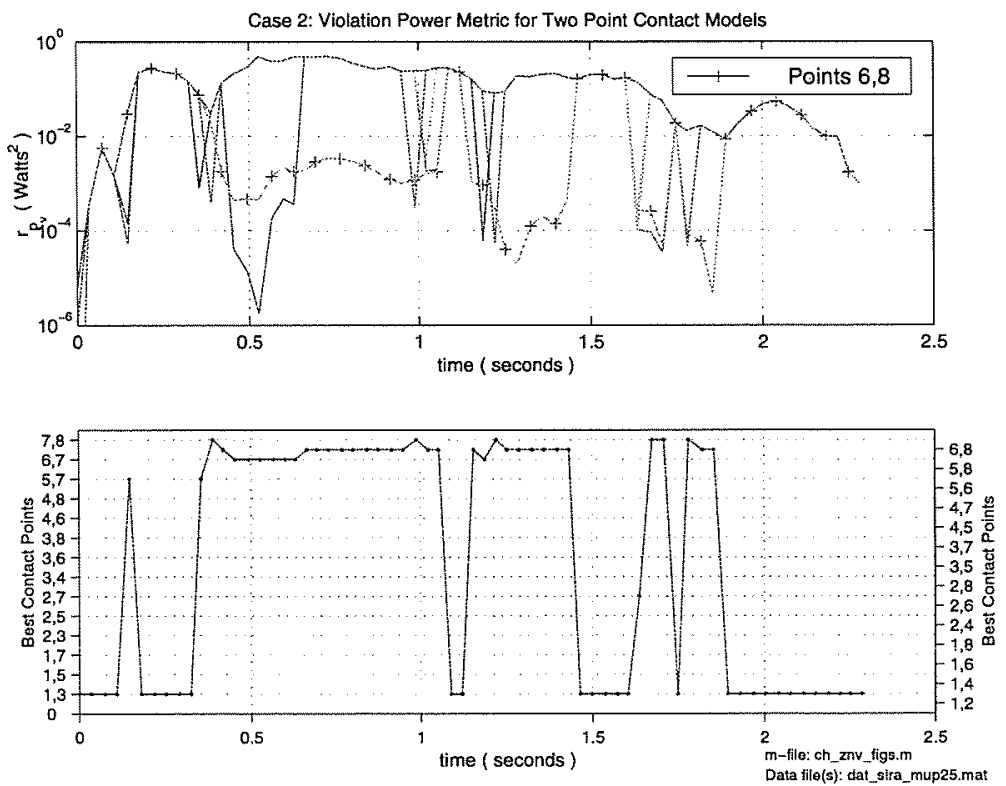

Figure C-5: Case 2: Best Two Point Model vs. Time. 

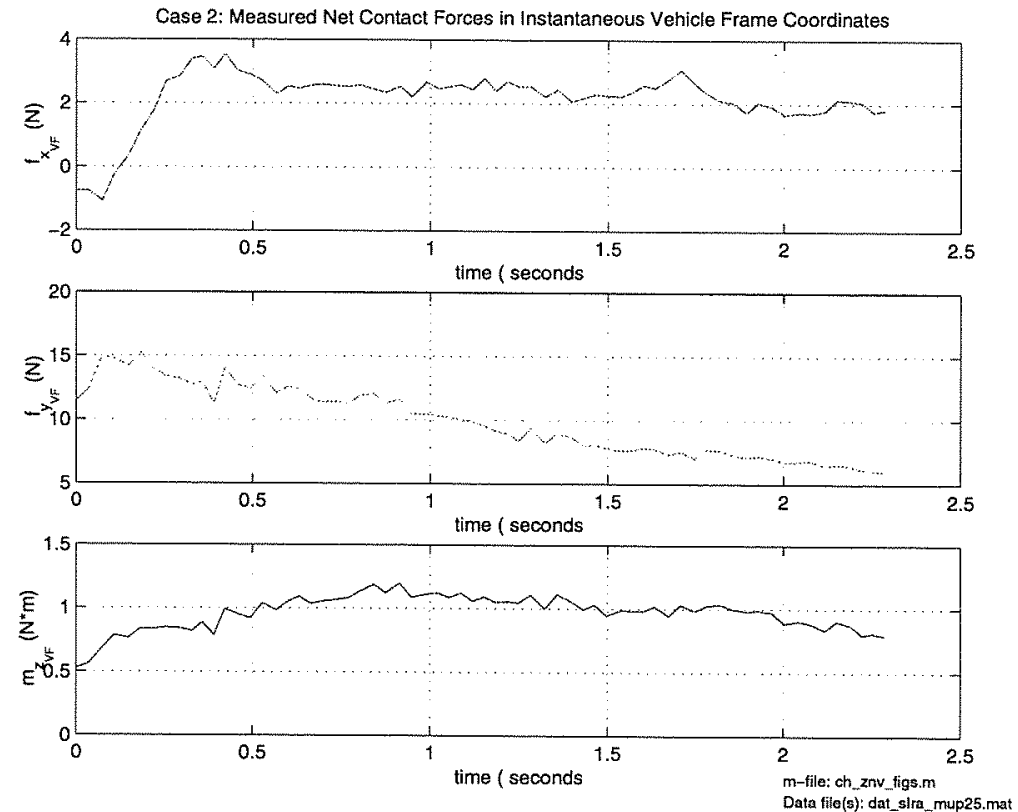

Figure C-6: Case 2: Measured Force and Moment vs. Time.
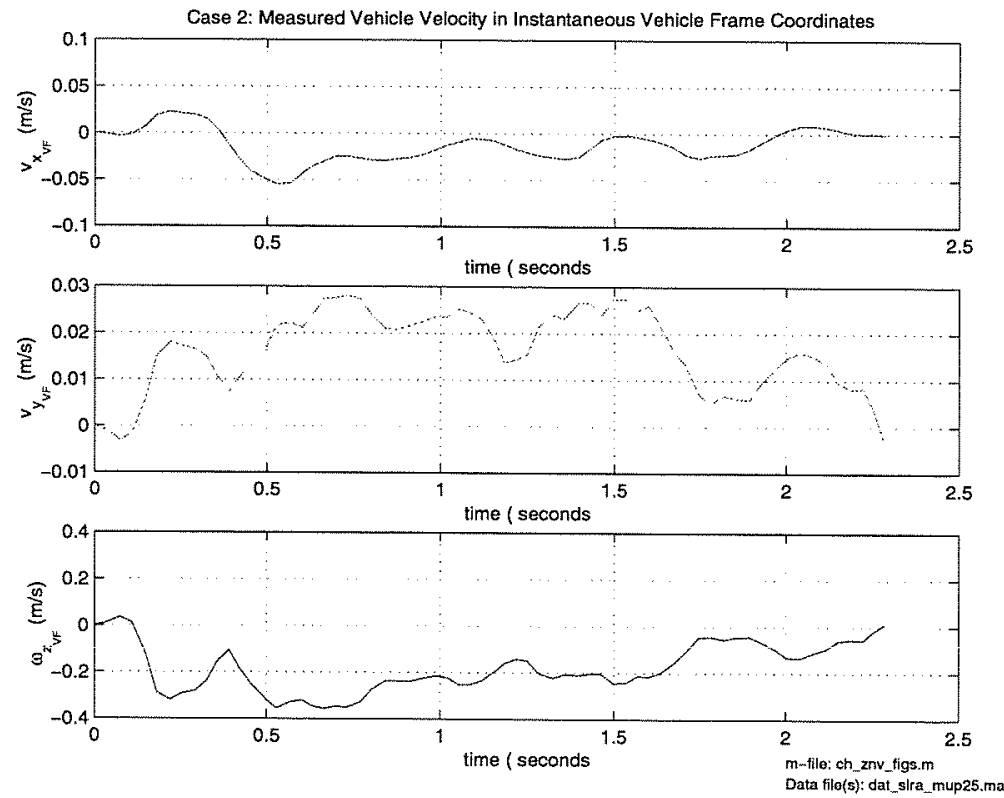

Figure C-7: Case 2: Measured Velocity vs. Time. 
Appendix D

Case 3: Two Point Contact ( Pure Translation ) 


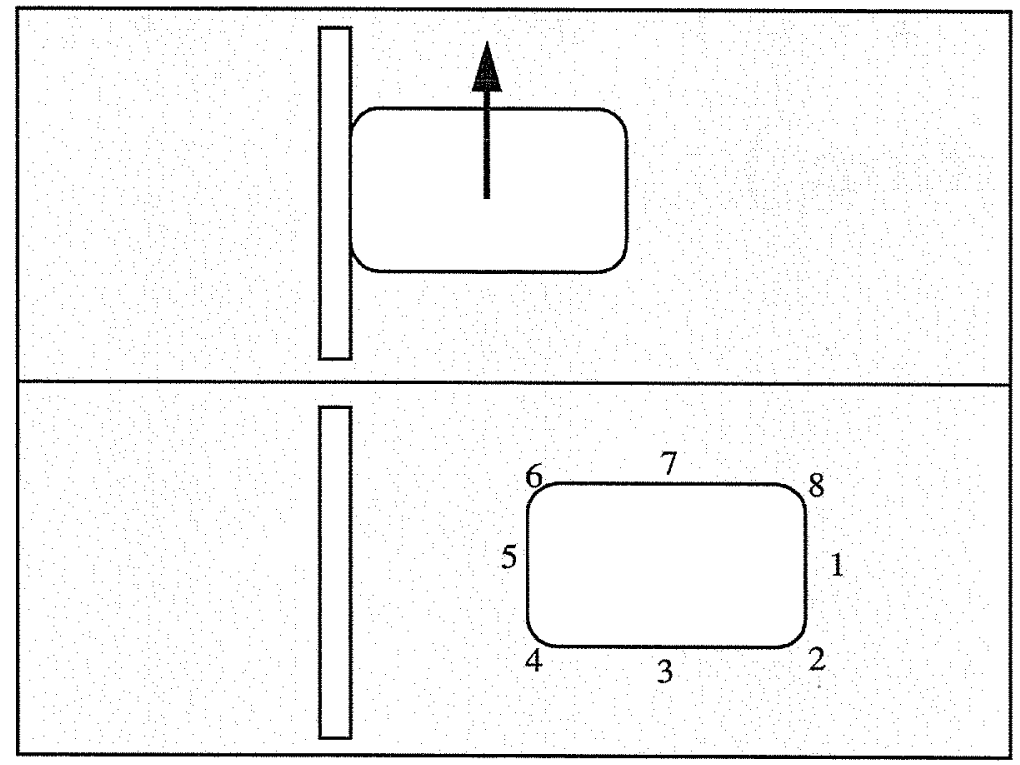

Figure D-1: Case 3 Pure Translation: Edge 5 of the vehicle was brought into full contact with a flat wall.. This contact was maintained throughout the trial as the vehicle was translated first upward and then downward back to its approximate initial position. The wall was smooth, rectangular block of 6061 aluminum 


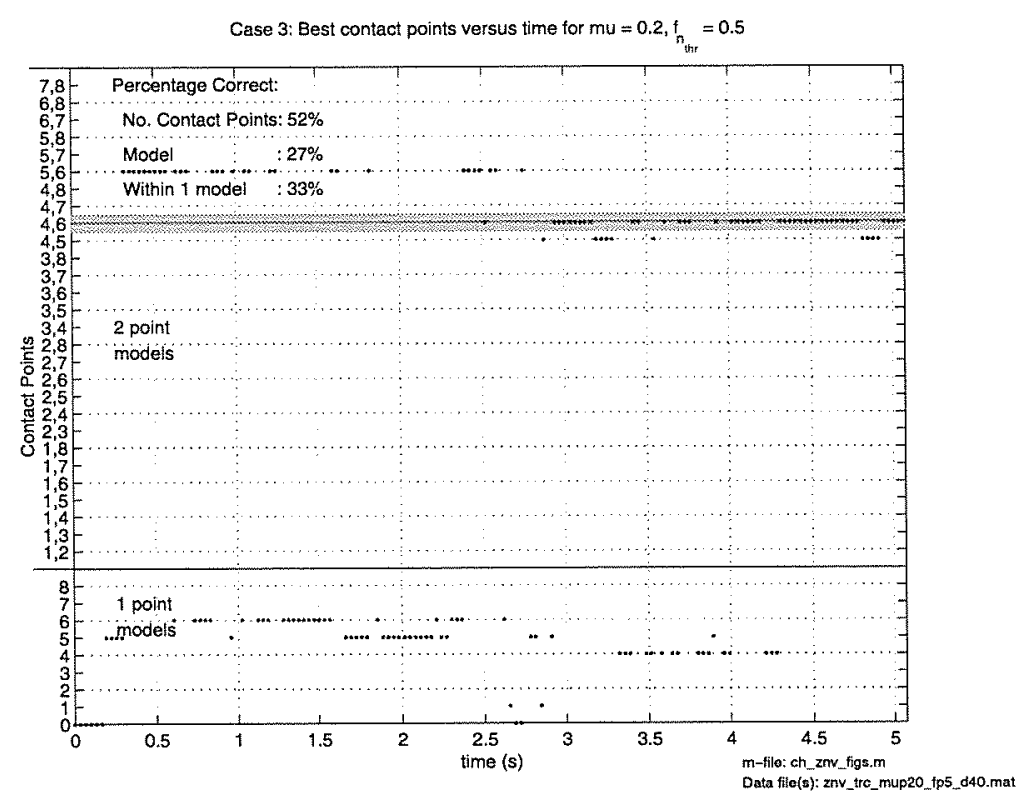

Figure D-2: Case 3: Best Overall Model vs. Time
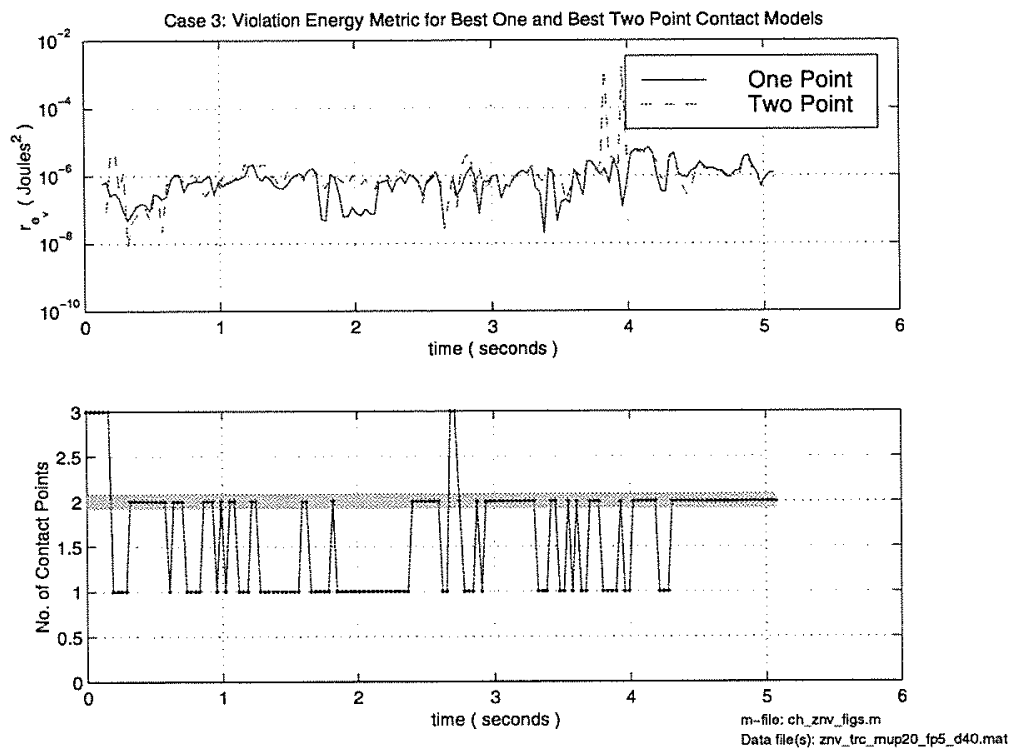

Figure D-3: Case 3: Best Number of Contact Points vs. Time 

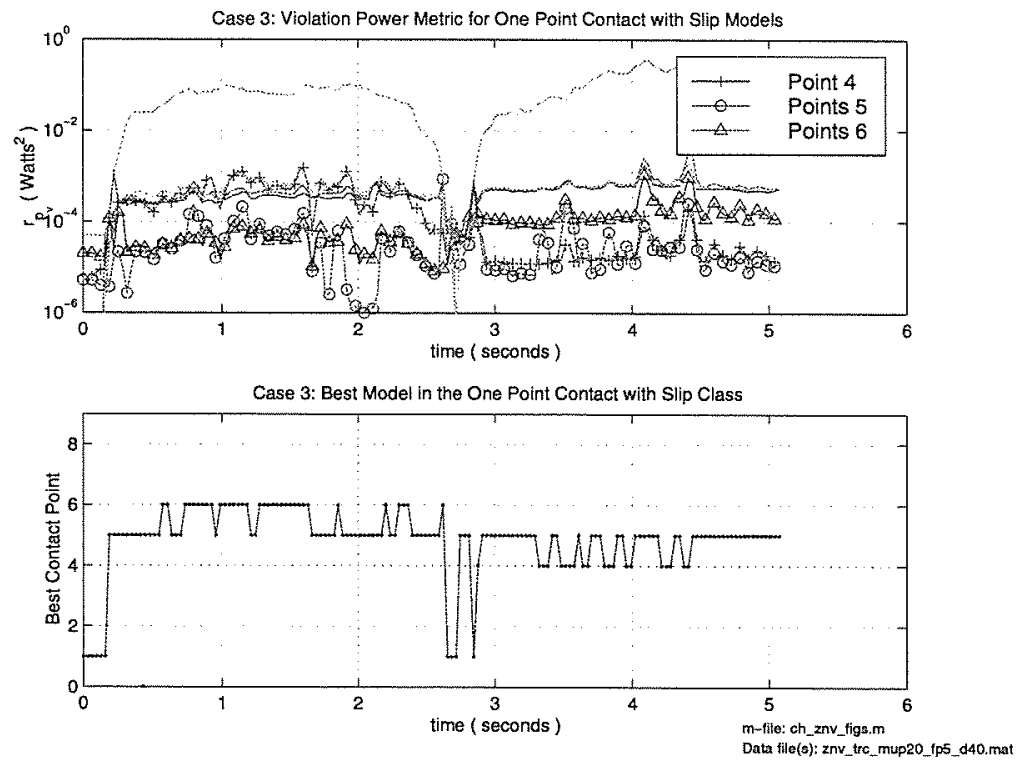

Figure D-4: Case 3: Best One Point Model vs. Time.
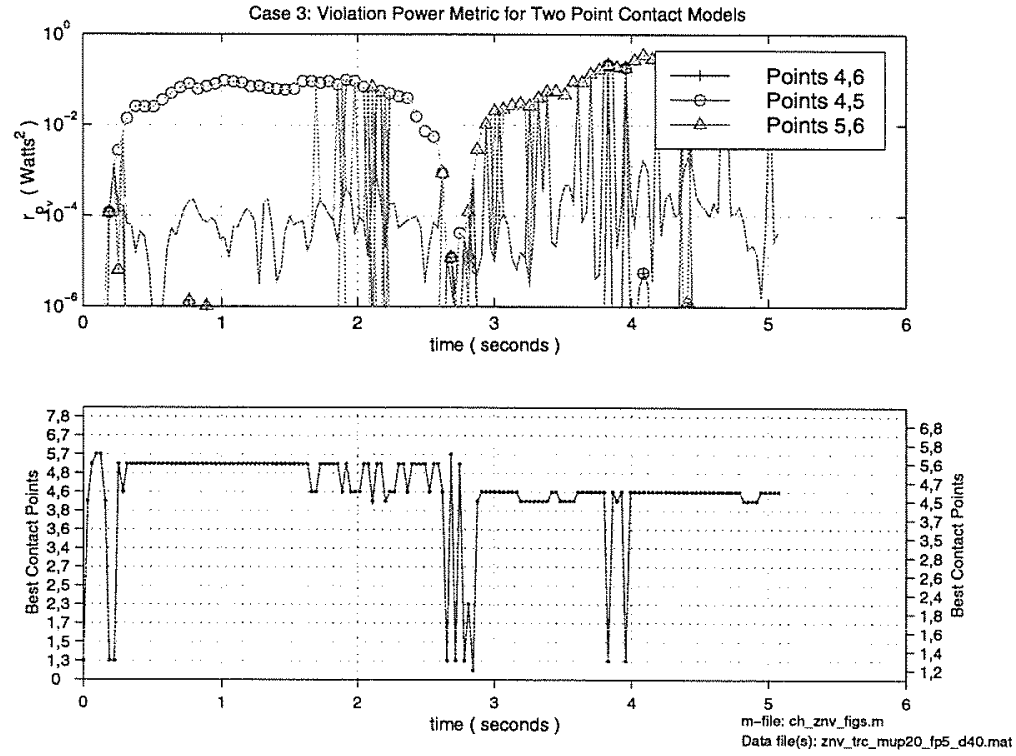

Figure D-5: Case 3: Best Two Point Model vs. Time. 

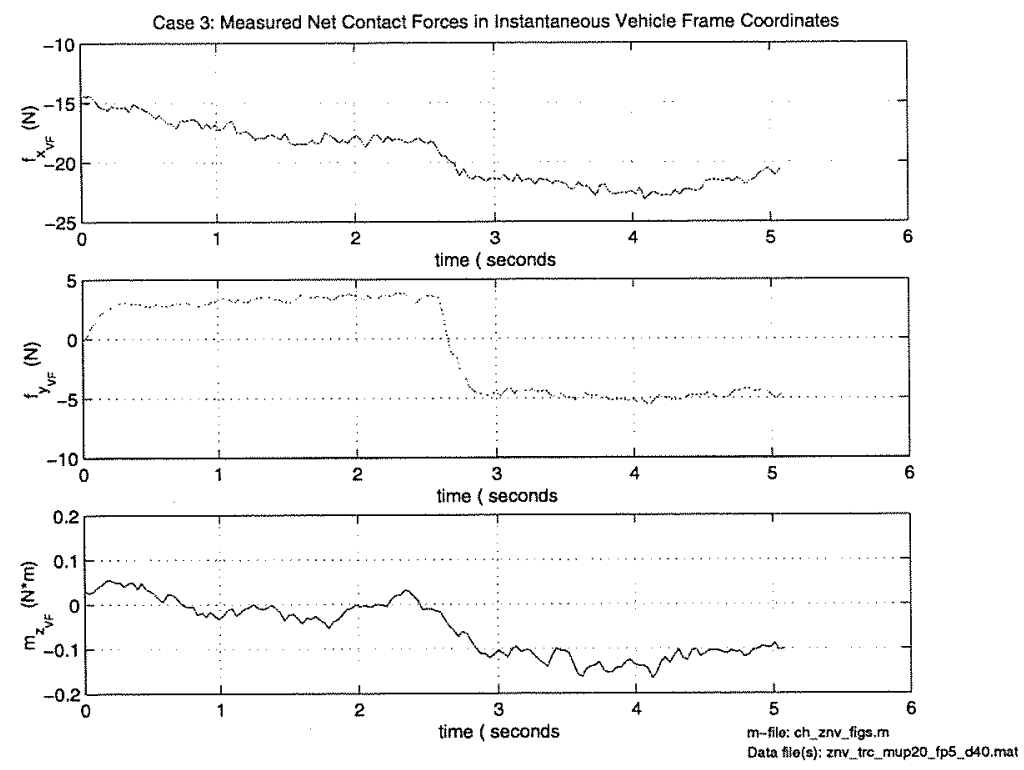

Figure D-6: Case 3: Measured Force and Moment vs. Time.
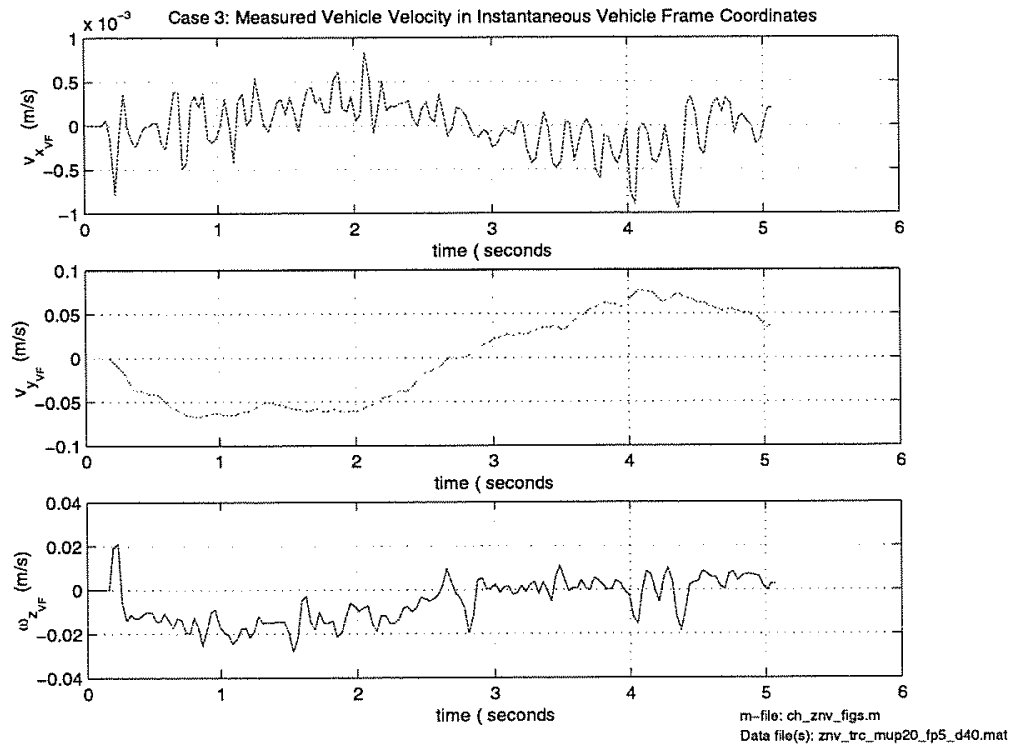

Figure D-7: Case 3: Measured Velocity vs. Time. 
Appendix E

\section{Case 4: Two Point Contact ( Rotation and Translation )}




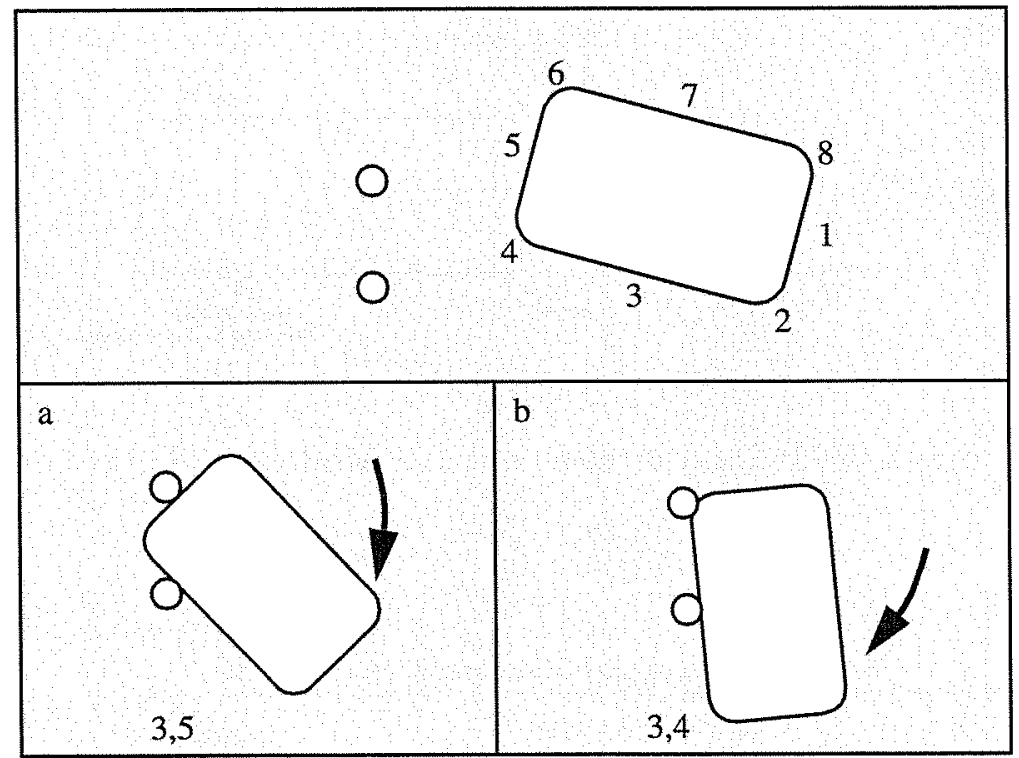

Figure E-1: Case 4 Experiment: The vehicle was brought into contact with two fixed cylindrical obstacles and then rotated in a clockwise direction. Initially vehicle edges 3 and 5 contacted the cylinders( we shall refer to this contact configuration as simply $(3,5))$. As the vehicle rotates, the actual contact state eventually transitions from from $(3,5)$ to $(3,4)$. Both obstacles were $12.7 \mathrm{~mm}$ diameter stainless steel bars. 


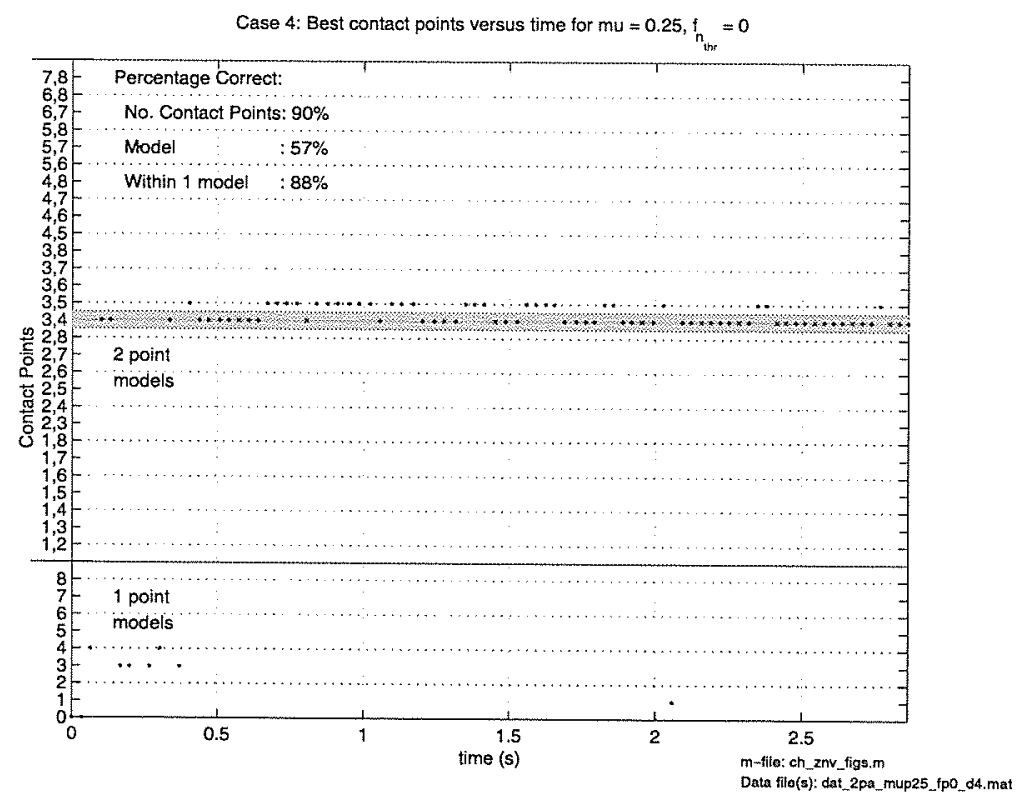

Figure E-2: Case 4: Best Overall Model vs. Time
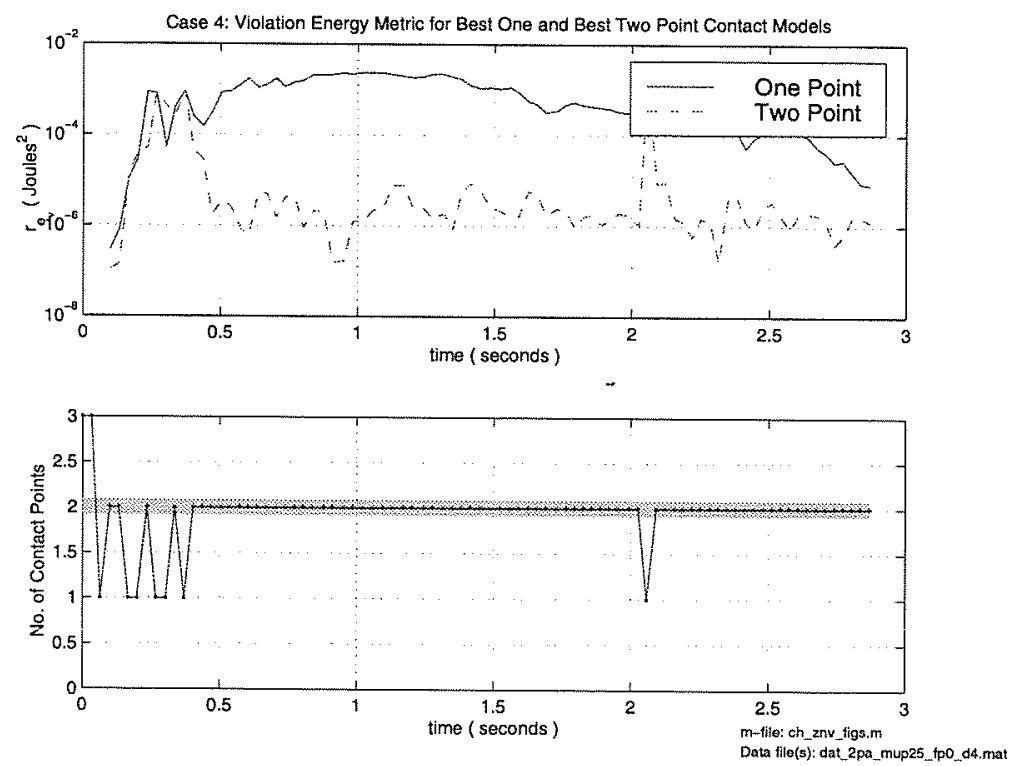

Figure E-3: Case 4: Best Number of Contact Points vs. Time 

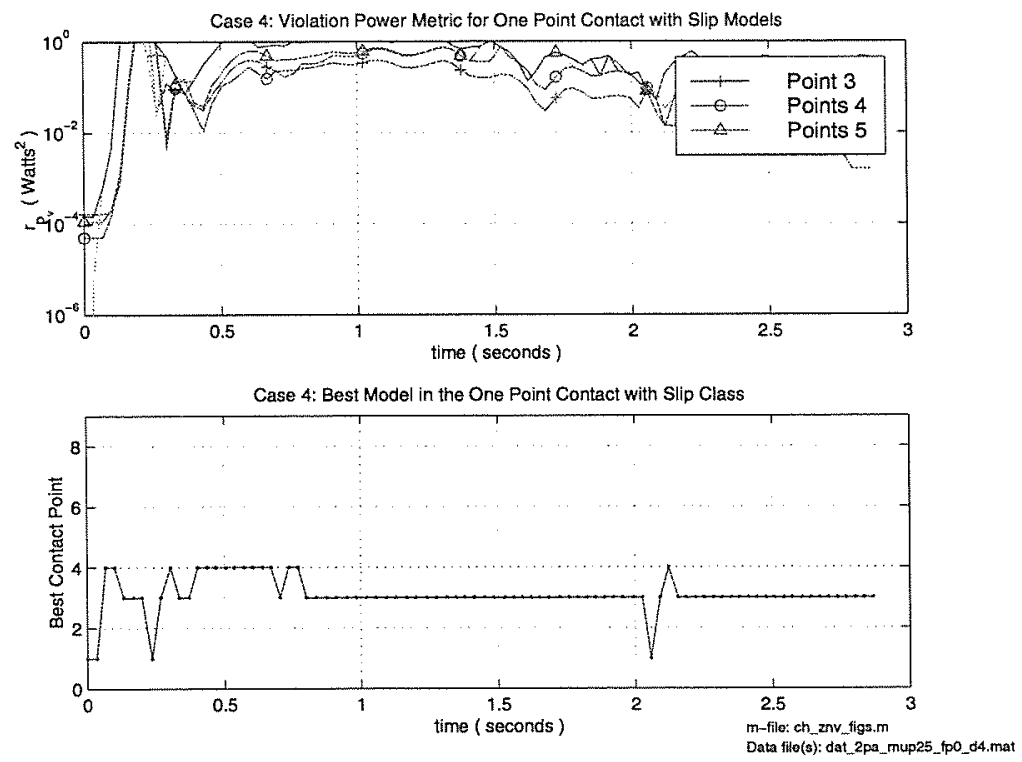

Figure E-4: Case 4: Best One Point Model vs. Time.
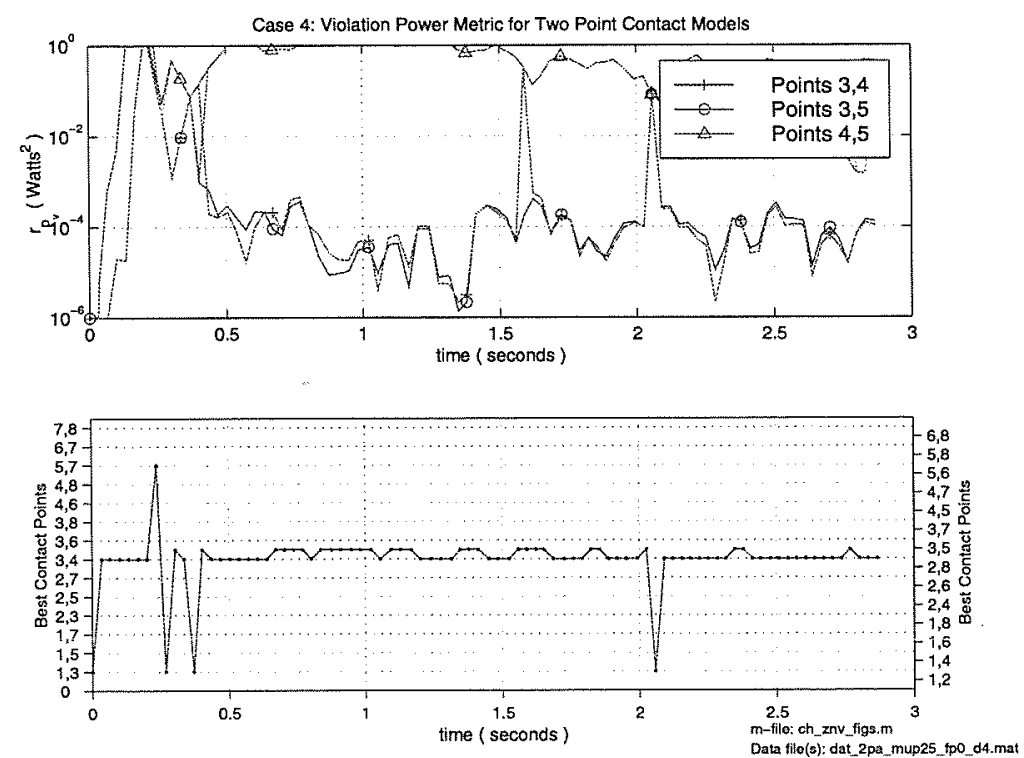

Figure E-5: Case 4: Best Two Point Model vs. Time. 

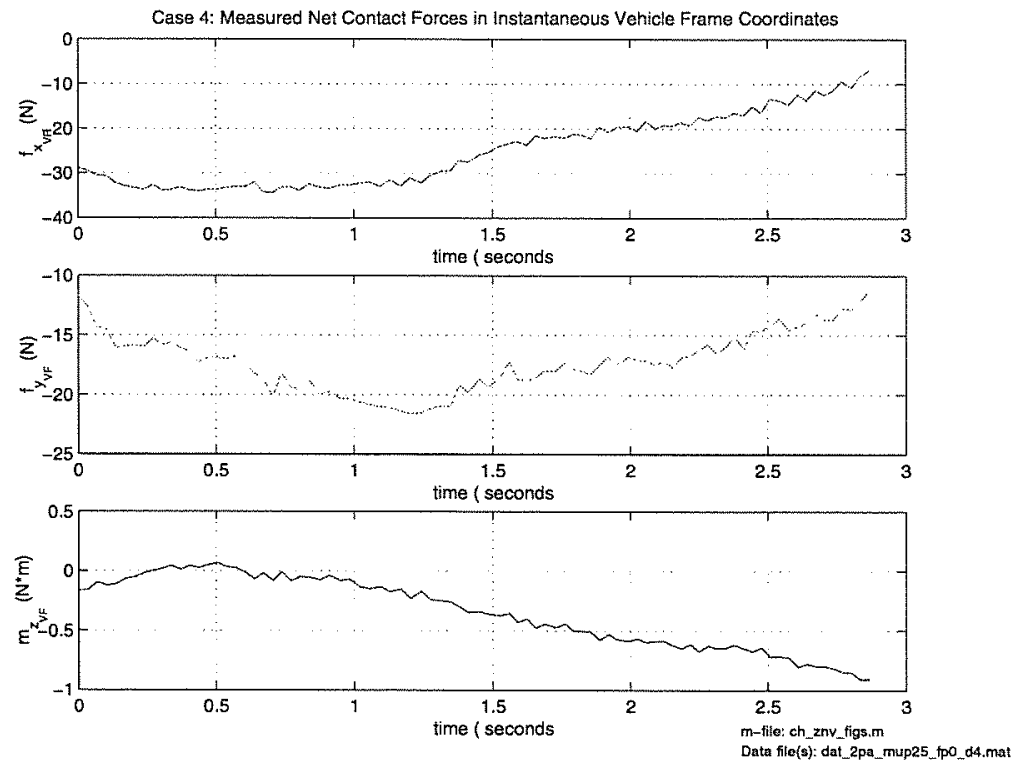

Figure E-6: Case 4: Measured Force and Moment vs. Time.
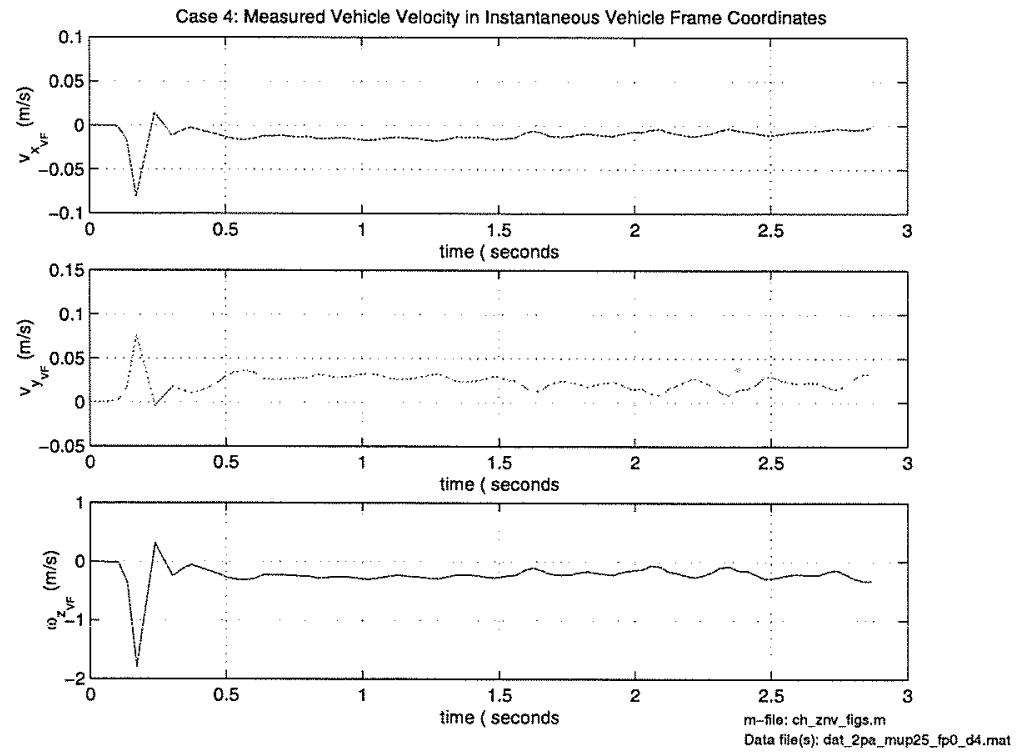

Figure E-7: Case 4: Measured Velocity vs. Time. 
Appendix F

Case 5: Two Point Contact ( Rotation and Translation ) 


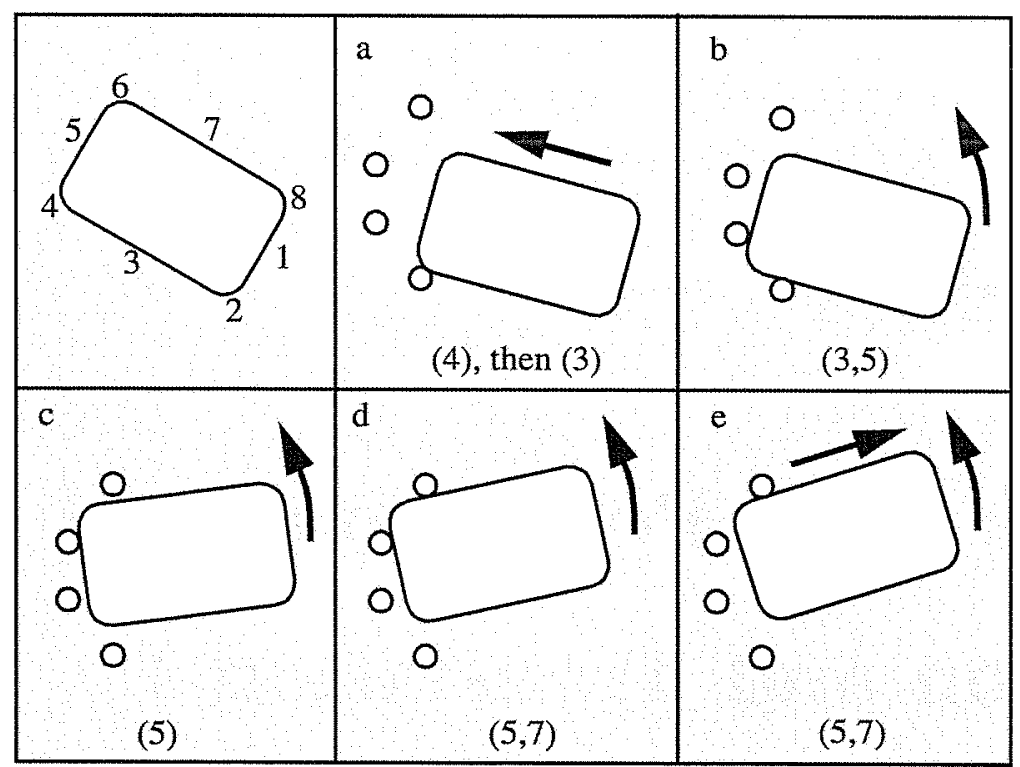

Figure F-1: Case 5 Experiment: The vehicle was brought into contact with a set of four fixed cylindrical obstacles. The vehicle was moved such that the sequence of contact states depicted above took place. All four obstacles were $12.7 \mathrm{~mm}$ diameter stainless steel bars.

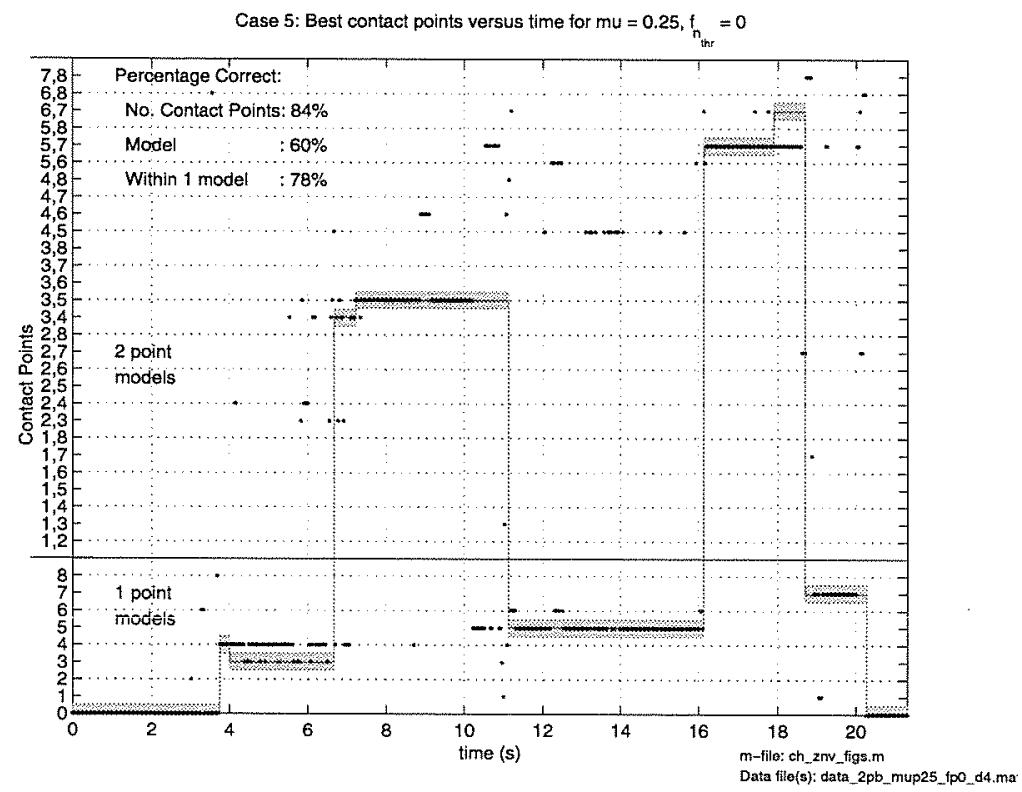

Figure F-2: Case 5: Best Overall Model vs. Time 

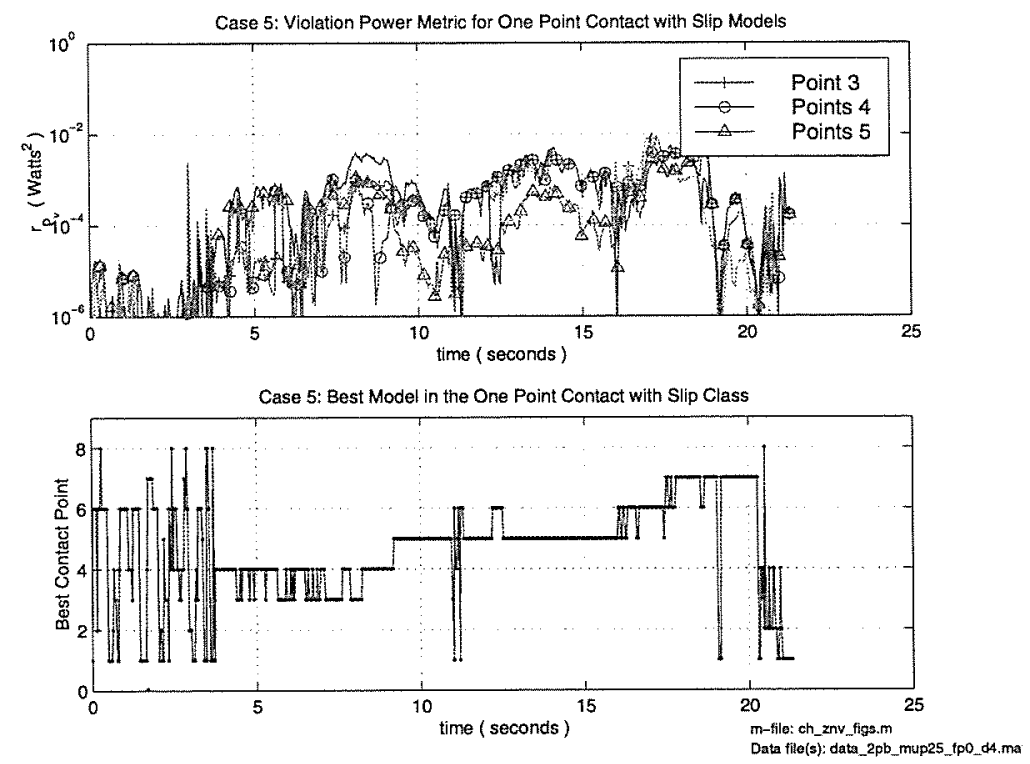

Figure F-3: Case 5: Best One Point Model vs. Time.
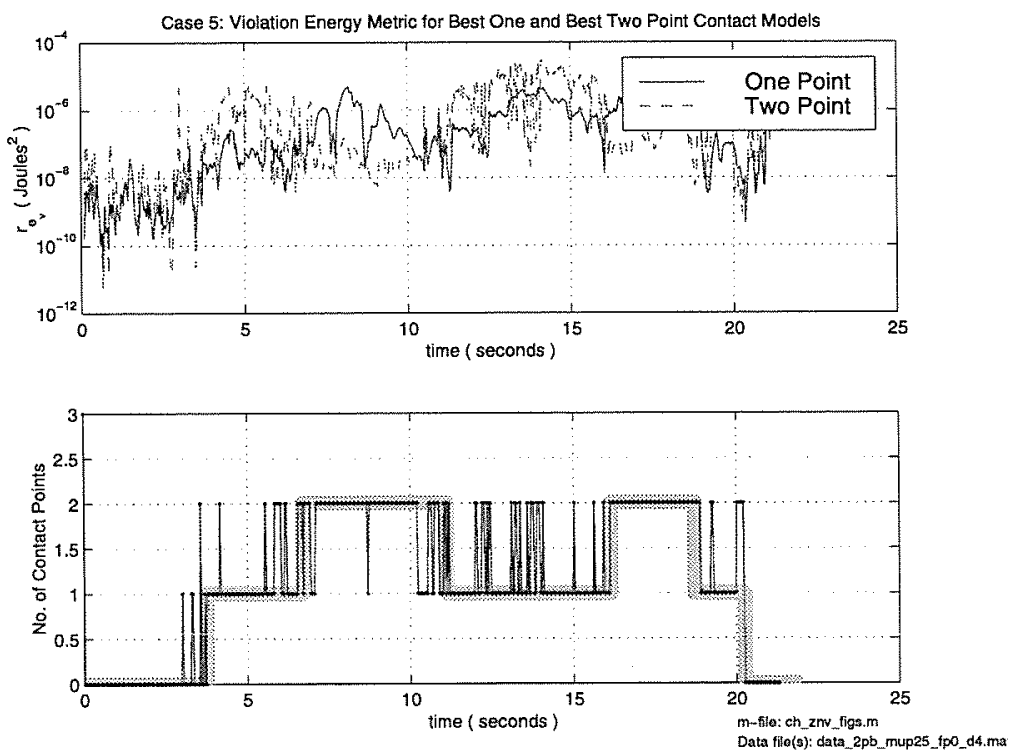

Figure F-4: Case 5: Best Number of Contact Points vs. Time 

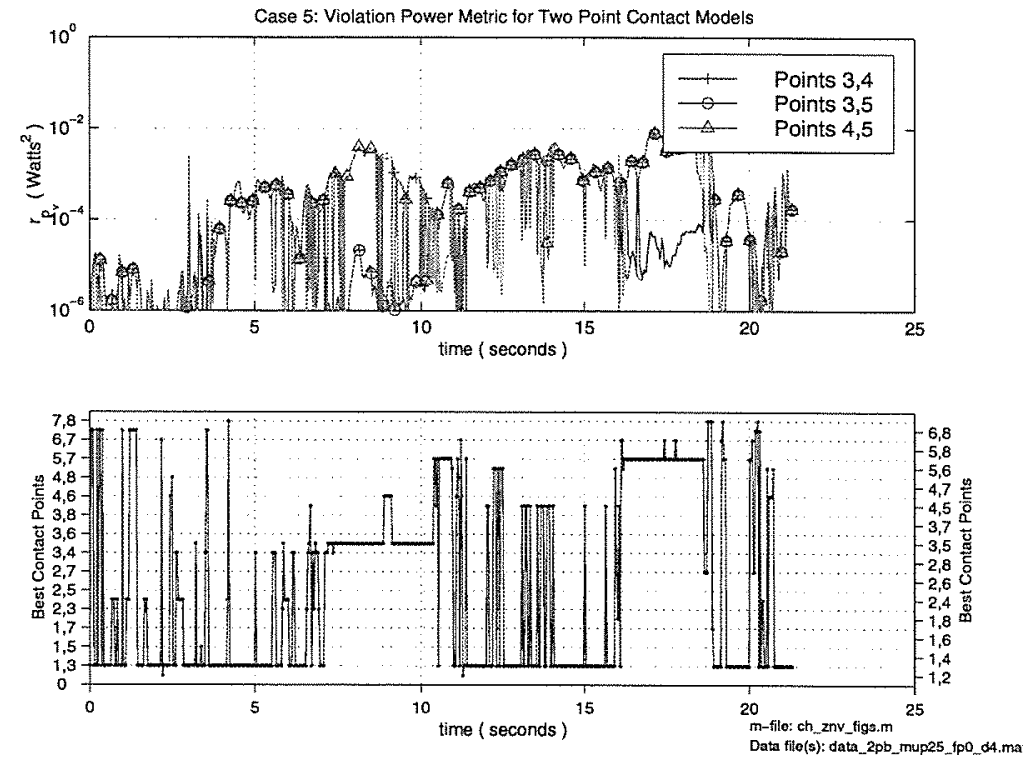

Figure F-5: Case 5: Best Two Point Model vs. Time.
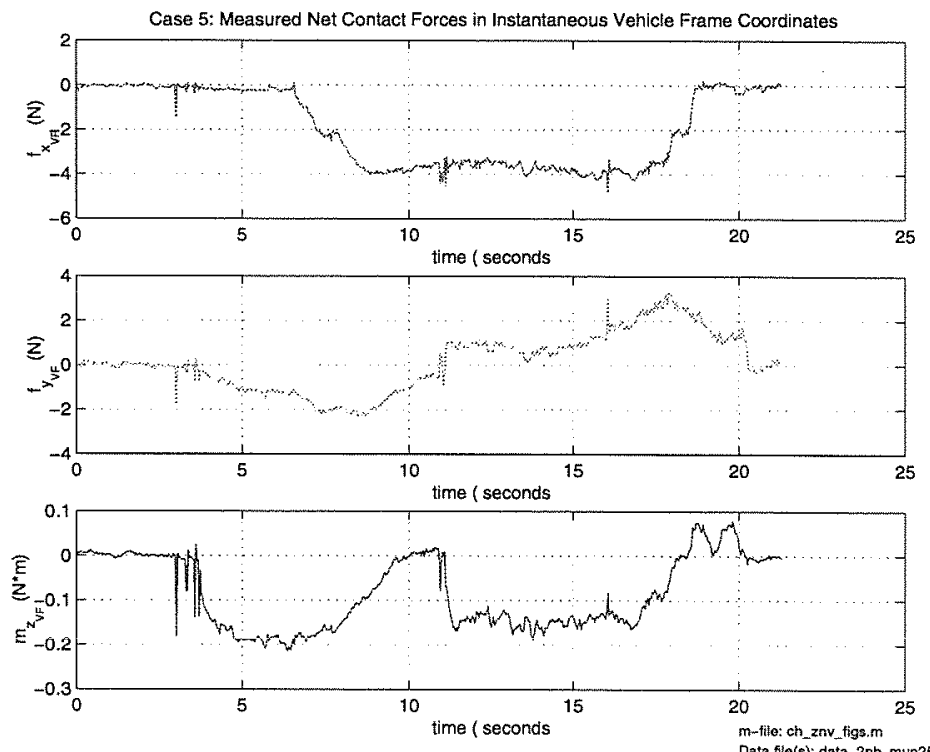

Figure F-6: Case 5: Measured Force and Moment vs. Time. 

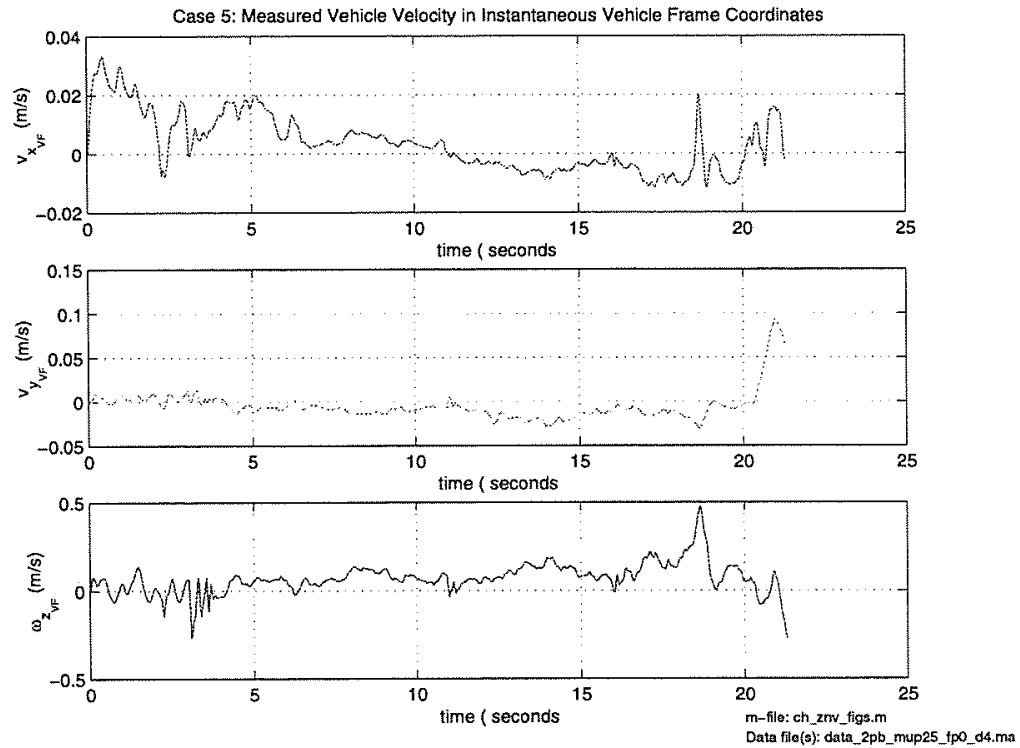

Figure F-7: Case 5: Measured Velocity vs. Time. 


\section{Appendix $G$}

\section{Consistency Measure and Dimenional Analysis}

Ideally our consistency measure would actually be a consistency metric, i.e. a single valued positive definite function whose value is zero if an only if none of the constraints associated with the the model being tested were violated at all. An obvious form for such a metric would be

$$
P=\mathcal{F}_{I}^{T} \mathbf{W}_{f} \mathcal{F}_{I}+\Delta \mathcal{X}_{I}^{T} \mathbf{W}_{\Delta x} \Delta \mathcal{X}_{I}
$$

where $\mathbf{W}_{f}$ and $\mathbf{W}_{\Delta x}$ are positve definite weighting matrices whose elements have the appropriate units to make $\mathrm{P}$ dimensionly consistent. The left-hand term is a measure of the degree to which the measured force vector $\mathcal{F}_{m}$ violates the constraints associated with the contact model being tested while the right-hand term indicates the degree to which the vehicle's measured differential motion vector $\Delta \boldsymbol{X}_{m}$ violates the model's motion constraints.

We know that, given perfect data, all of these constraints would be perfectly satified when we tested the correct model. With imperfect sensors and imperfect 
knowledge of the vehicle's physical parameters we expect that, in the real world, even the correct model's constraints will not be perfectly satisfied. However, we expect that the constraint violations for the correct model will be smaller than those for incorrect models and so we choose the model with the smallest inconsisitency measure.

We face a problem in constructing an inconsistency measure, however. Each candidate model has six constraints associated with it; two force constraints, one moment constraint, two linear motion constraints and one angular motion constraint. While we can easily compute to what degree each individual constraint is violated, coming up with an overall in consistency measure for the model is complicated by the fact that these violations have a variety of different units (i.e. force, moment, linear displacement, angular displacement). To intelligently combine these different terms into a single, dimensionally sensible measure we must not only convert each term into a common set of units but we must also determine the relative importance of each constraint.

We can employ dimensional analysis to express the different terms in the same units (i.e. express each term in dimensionless form). It The elements of the vectors $\mathcal{F}_{I}$ and $\Delta \mathcal{X}_{I}$ have units of

$$
\begin{array}{rr}
{\left[f_{x}\right]=\left[\frac{L M}{T^{2}}\right],} & {[\Delta x]=[L]} \\
{\left[f_{y}\right]=\left[\frac{L M}{T^{2}}\right],} & {[\Delta y]=[L]} \\
{\left[m_{V}\right]=\left[\frac{L^{2} M}{T^{2}}\right],} & {[\Delta \theta]=[1]}
\end{array}
$$

where, using Buckingham Pi notation, surrounding a term $g$ by square brackets means "the units of $g$ " and the terms $L, M$ and $T$ represent units quantities having the dimensions of length, mass and time respectively. Thus we have five independent measured quanties which are not dimensionless $\left(f_{x}, f_{y}, m_{V}, \Delta x, \Delta y\right)$ but only theree WHATEVER parameters ( $L, M$ and $T$ ), indicating that we need only $5-3=2$ 
WHATEVER parameters to non-dimensionalize the problem

$$
\begin{aligned}
f_{x}^{*} & =\frac{f_{x}}{f_{C}} \\
f_{y}^{*} & =\frac{f_{y}}{f_{C}} \\
m_{V}^{*} & =\frac{m_{V}}{f_{C} l_{C}} \\
\Delta x^{*} & =\frac{\Delta_{x}}{l_{C}} \\
\Delta y^{*} & =\frac{\Delta_{y}}{l_{C}} \\
\Delta \theta^{*} & =\Delta \theta
\end{aligned}
$$

where $f_{C}$ and $l_{C}$ are a characteristic force and a characteristic length, respectively, that we must choose. Unfortunately the choice of these parameters is arbitrary and as such results in the assignment of arbitrary relative weights to the various constraints associated with the model. This is very undesirable as it requires an ad hoc approach to finding values of $f_{C}$ and $l_{C}$ lead to accurate contact identification and, if such values can be found, there is no guarantee that they will work well for contact situations other than the once used to obtain the values. Thus an inconsistency metric of the form of equation G.1 is not an advisable choice. 


\section{Appendix $\mathbf{H}$}

\section{Reaction loads for two point}

\section{contact models, assuming $\mu_{d}$}

\section{unknown}

$$
\begin{aligned}
\Sigma F_{x}=0= & f_{x_{e x t}}+f_{n_{i}} \mu_{d} \operatorname{sign}\left(v_{t_{i}}\right)+f_{n_{j}} \mu_{d} \operatorname{sign}\left(v_{t_{j}}\right) \cos \theta_{j}-f_{n_{j}} \sin \theta_{j} \\
\Sigma F_{y}=0= & f_{y_{e x t}}+f_{n_{i}}+f_{n_{j}} \mu_{d} \operatorname{sign}\left(v_{t_{j}}\right) \sin \theta_{j}+f_{n_{j}} \cos \theta_{j} \\
\Sigma m_{p_{i}}=0= & M_{e x t}-y_{j} f_{n_{j}}\left[\mu_{d} \operatorname{sign}\left(v_{t_{j}}\right) \cos \theta_{j}-\sin \theta_{j}\right] \\
& +x_{j} f_{n_{j}}\left[\mu_{d} \operatorname{sign}\left(v_{t_{j}}\right) \sin \theta_{j}+\cos \theta_{j}\right]
\end{aligned}
$$

$$
\begin{aligned}
0= & M_{e x t}-f_{n_{j}} \mu_{d} \operatorname{sign}\left(v_{t_{j}}\right)\left[-x_{j} \sin \theta_{j}+y_{j} \cos \theta_{j}\right] \\
& +f_{n_{j}}\left[x_{j} \cos \theta_{j}+y_{j} \sin \theta_{j}\right] \\
0= & M_{e x t}+f_{n_{j}}\left[X-Y \mu_{d} \operatorname{sign}\left(v_{t_{j}}\right)\right]
\end{aligned}
$$


Solve H.2 for $f_{n_{i}}$ to get

$$
f_{n_{i}}=-\frac{\left[f_{x_{e x t}}+f_{n_{j}}\left(\mu_{d} \operatorname{sign}\left(v_{t_{j}}\right) \cos \theta_{j}-\sin \theta_{j}\right)\right]}{\mu_{d} \operatorname{sign}\left(v_{t_{i}}\right)}
$$

Likewise, solve H.3 for $f_{n_{i}}$ to get

$$
f_{n_{i}}=-\left[f_{y_{e x t}}+f_{n_{j}}\left(\mu_{d} \operatorname{sign}\left(v_{t_{j}}\right) \sin \theta_{j}+\cos \theta_{j}\right)\right]
$$

Equating these results and solving for $f_{n_{j}}$ we obtain

$$
\begin{aligned}
f_{n_{j}} & =\frac{f_{x_{e x t}}-f_{y_{e x t}} \mu_{d} \operatorname{sign}\left(v_{t_{i}}\right)}{\mu_{d}^{2} \operatorname{sign}\left(v_{t_{i}} v_{t_{j}}\right) \sin \theta_{j}+\mu_{d} \operatorname{sign}\left(v_{t_{i}}\right) \cos \theta_{j}-\mu_{d} \operatorname{sign}\left(v_{t_{j}}\right) \cos \theta_{j}+\sin \theta_{j}} \\
f_{n_{j}} & =\frac{f_{x_{e x t}}-f_{y_{e x t}} \mu_{d} \operatorname{sign}\left(v_{t_{i}}\right)}{a \mu_{d}^{2}+b \mu_{d}+c}
\end{aligned}
$$

where

$$
\begin{aligned}
a & =\operatorname{sign}\left(v_{t_{i}} v_{t_{j}}\right) \sin \theta_{j} \\
b & =\left[\operatorname{sign}\left(v_{t_{i}}\right)-\operatorname{sign}\left(v_{t_{j}}\right)\right] \cos \theta_{j} \\
c & =\sin \theta_{j}
\end{aligned}
$$

Using H.7 to replace $f_{n_{j}}$ in equation H.6 the expression for $f_{n_{j}}$ becomes

$$
f_{n_{i}}=-f_{y_{e x t}}+\frac{\left(f_{y_{e x t}} \mu_{d} \operatorname{sign}\left(v_{t_{i}}\right)-f_{x_{e x t}}\right)\left(\mu_{d} \operatorname{sign}\left(v_{t_{j}}\right) \sin \theta_{j}+\cos \theta_{j}\right)}{a \mu_{d}^{2}+b \mu_{d}+c}
$$

Combining both terms over a common denominator and simplifying yields

$$
f_{n_{i}}=\frac{f_{y_{e x t}}\left[\mu_{d} \operatorname{sign}\left(v_{t_{j}}\right) \cos \theta_{j}+\sin \theta_{j}\right]-f_{x_{e x t}}\left[\mu_{d} \operatorname{sign}\left(v_{t_{j}}\right) \sin \theta_{j}+\cos \theta_{j}\right]}{a \mu_{d}^{2}+b \mu_{d}+c}
$$

Using H.12 and H.11 to replace $f_{n_{i}}$ and $f_{n_{j}}$ in equation H.3 and combining terms 
yields a quadratic in $\mu_{d}$, that is

$$
A \mu_{d}^{2}+B \mu_{d}+C=0
$$

where

$$
\begin{aligned}
& A=\operatorname{sign}\left(v_{t_{i}} v_{t_{j}}\right)\left[M_{e x t} \sin \theta_{j}+Y f_{y_{e x t}}\right] \\
& B=\operatorname{sign}\left(v_{t_{i}}\right)\left[M_{e x t} \cos \theta_{j}-X f_{y_{e x t}}\right]-\operatorname{sign}\left(v_{t_{j}}\right)\left[M_{e x t} \cos \theta_{j}+Y f_{x_{e} x t}\right] \\
& C=M_{e x t} \sin \theta_{j}+X f_{x_{e x t}}
\end{aligned}
$$

Thus, to find the reaction loads at the contact points we find the two solutions to H.13 and use H.12 and H.11 to compute the normal reaction loads associated with each solution.

Because there are two solutions to H.13, each pairing of candidate contact points yields, in effect, two contact models, one associated with each solution of H.13. 


\section{Appendix I}

\section{Mathematical Notation for Part II}

\section{Scalar, Vector and Matrix Variables}

- Scalar variables are represented by italicized symbols ( e.g. $\omega, n, f_{n}$, etc.).

- Vector and matrix variables are represented by bold face symbols (e.g. $\mathbf{r}_{i}, \mathbf{R}_{i}$, A, etc.).

- Vectors whose elements have differing units are represented by bold face capitalized calligraphic symbols (e.g. $\mathcal{F}_{m}, \mathcal{V}_{I}, \Delta \mathcal{X}_{P}$, etc).

- Subscripts on scalar, vector and matrix variables specific instances of generic variables (e.g. $\mu_{d}, \mu_{s}, \mathcal{F}_{m}, \mathcal{F}_{P}, \mathcal{F}_{I}$, etc. ).

- Prefixed superscripts specify the reference frame the quantity in which the quantity is represented.

\section{Planar Cross Product}

In general three dimensional (i.e. non-planar) motion, the linear velocity $\mathcal{V}_{I}$ of a point $p_{i}$ on a rigid body can be wrritten as

$$
\mathbf{v}_{i}=\mathbf{v}_{j}+\mathbf{r}_{j i} \times \omega
$$




\begin{tabular}{|c|c|c|c|}
\hline Symbol & Dimension & Units & Description \\
\hline$N$ & Scalar $1 \times 1$ & none & number of candidate contact points \\
\hline $\mathcal{F}_{m}$ & Vector $3 \mathrm{x} 1$ & $\mathrm{~N}, \mathrm{Nm}$ & measured contact force and moment \\
\hline $\mathcal{F}_{P}$ & Vector $3 \mathrm{x} 1$ & $\mathrm{~N}, \mathrm{Nm}$ & $\begin{array}{l}\text { permissable portion of measured } \\
\text { force vector }\end{array}$ \\
\hline $\mathcal{F}_{I}$ & Vector $3 \times 1$ & $\mathrm{~N}, \mathrm{Nm}$ & $\begin{array}{l}\text { impermissable portion of measured } \\
\text { force vector }\end{array}$ \\
\hline $\mathcal{V}_{m}$ & Vector $3 \times 1$ & $\mathrm{~m} / \mathrm{s}, \mathrm{rad} / \mathrm{sec}$ & measured vehicle velocity \\
\hline$\Delta \mathcal{X}_{m}$ & Vector $3 \times 1$ & $\mathrm{~m}, \mathrm{rad}$ & measured differential motion vector \\
\hline$\Delta \mathcal{X}_{P}$ & Vector $3 \times 1$ & $\mathrm{~N}, \mathrm{Nm}$ & $\begin{array}{l}\text { permissable portion of differential } \\
\text { motion vector }\end{array}$ \\
\hline$\Delta \mathcal{X}_{I}$ & Vector $3 \times 1$ & $\mathrm{~N}, \mathrm{Nm}$ & $\begin{array}{l}\text { impermissable portion of differential } \\
\text { motion vector }\end{array}$ \\
\hline$\overline{\mathbf{r}_{i}}$ & Vector $2 \times 1$ & $\mathrm{~m}$ & position vector to candidate contact point $i$ \\
\hline$\mu_{d}$ & Scalar 1x1 & none & dynamic coefficient of friction \\
\hline$v_{t}$ & Scalar $1 \times 1$ & $\mathrm{~m} / \mathrm{s}$ & velocity in tangential direction \\
\hline$v_{n}$ & Scalar 1x1 & $\mathrm{m} / \mathrm{s}$ & velocity in normal direction \\
\hline$\omega$ & Scalar $1 \times 1$ & $\mathrm{rad} / \mathrm{s}$ & angular velocity \\
\hline$f_{t}$ & Scalar $1 \mathrm{x} 1$ & $\mathrm{~N}$ & contact force in tangential direction \\
\hline$f_{n}$ & Scalar 1x1 & $\mathrm{N}$ & contact force in normaldirection \\
\hline I & Matrix & none & identity matrix \\
\hline $\mathbf{R}_{\mathrm{i}}$ & Matrix $2 \times 2$ & none & rotation matrix \\
\hline$n$ & Scalar $1 \times 1$ & none & $\begin{array}{l}\text { no. of independent variables required to } \\
\text { describe a candiate contact model's } \\
\text { contact point reaction loads }\end{array}$ \\
\hline $\mathrm{A}$ & Matrix $3 \times \mathrm{xm}$ & none, $\mathrm{m}$ & $\begin{array}{l}\text { coefficients of a candidate contact model's } \\
\text { force constraint equations }\end{array}$ \\
\hline $\mathrm{B}$ & Matrix $3 x(3-m)$ & none, $\mathrm{m}$ & $\begin{array}{l}\text { coefficients of a candidate contact model's } \\
\text { velocity constraint equations }\end{array}$ \\
\hline $\mathrm{P}_{\mathcal{F}_{P}}$ & Matrix $3 \times 3$ & none, $m$ & $\begin{array}{l}\text { projection matrix which extracts } \mathcal{F}_{P} \\
\text { from } \mathcal{F}_{m}\end{array}$ \\
\hline $\mathbf{I}-\mathbf{P}_{\mathcal{F}_{P}}$ & Matrix $3 \times 3$ & none, $\mathrm{m}$ & $\begin{array}{l}\text { projection matrix which extracts } \mathcal{F}_{I} \\
\text { from } \mathcal{F}_{m}\end{array}$ \\
\hline $\mathbf{P}_{\mathcal{V}_{P}}$ & Matrix $3 \times 3$ & none, $m$ & $\begin{array}{l}\text { projection matrix which extracts } \Delta \mathcal{X}_{P} \\
\text { from } \Delta \mathcal{X}_{m}\end{array}$ \\
\hline $\mathrm{I}-\mathbf{P}_{\mathcal{V}_{P}}$ & Matrix $3 \times 3$ & none, $\mathrm{m}$ & $\begin{array}{l}\text { projection matrix which extracts } \Delta \mathcal{X}_{I} \\
\text { from } \Delta \mathcal{X}_{m}\end{array}$ \\
\hline
\end{tabular}

Table I.1: Mathematical notation 
where $\mathbf{v}_{j}$ is the $3 \times 1$ linear velocity vector of some other point $p_{j}$ on the body, $\mathbf{r}_{j i}$ is the $3 \mathrm{x} 1$ displacement vector from point $p_{j}$ to $p_{i}$ and $\omega$ is the $3 \mathrm{x} 1$ angular velocity vector for the body. In planar motion, however, the angular velocity of a body is most conveniently described by a scalar $\omega$. For notational convenience we make the following definition. Given a $2 \times 1$ vector $\mathbf{r}_{i}$ we a define a $2 \times 1$ cross product vector $\left[\mathbf{r}_{i} \times\right]$ such that

$$
\left[\mathbf{r}_{i} \times\right]=\left[\begin{array}{c}
-r_{i_{y}} \\
r_{i_{x}}
\end{array}\right]
$$

where $r_{i_{x}}$ and $r_{i_{y}}$ are the scalar $x$ and $y$ components of the original vector $\mathbf{r}_{i}$. Given this definition, the planar version of the cross product between a vector $\mathbf{r}_{i}$ and the angular velocity is

$$
\mathbf{r}_{i} \times \omega=\left[\mathbf{r}_{i} \times\right] \omega
$$

Using the same notation, the cross product between a vector $\mathbf{r}_{i}$ and a second $2 \mathrm{x} 1$ vector $\mathbf{v}$ can be written as

$$
\mathbf{r}_{i} \times \mathbf{v}=\left[\mathbf{r}_{i} \times\right]^{T} \mathbf{v}
$$

We shall use this notation throughout this thesis. 


\section{References}

[1] H.L. Alexander and R.H. Cannon. An extended operational-space algorithm for satellite manipulators. Journal of the Astronautical Sciences, 38(4):473-486, 1990.

[2] H. Asada and A.B. By. Kinematic analysis of workpart fixturing for flexible assembly with automatically reconfigurable fixtures. IEEE Transactions on Robotics and Automation, RA-1(2):86-94, June 1985.

[3] H. Asada and J.J. Slotine. Robot Analysis and Control. Wiley and Sons, 1986.

[4] J.J. Bausch. Kinematic Methods for Automated Fixture Design. PhD thesis, MIT, January 1990. Dept. of Mech. Engineering.

[5] A. Bicci, J.K. Salisbury, and D.L. Brock. Contact sensing from force measurements. The International Journal of Robotics Research, 12(3):249-262, June 1993.

[6] R.C. Brost and K.Y. Goldberg. A complete algorithm for designing planar fixtures using modular components. IEEE Transactions on Robotics and Automation, 12(1):31-46, February 1996.

[7] H. Bruyninckx. Kinematic Models for Robot Compliant Motion with Identification of Uncertainties. PhD thesis, Katholieke Universiteit Leuven, April 1995. Dept. of Mech. Engineering. 
[8] S. Dubowsky and E. Papadopoulos. The kinematics, dynamics, and control of free-flying and free-floating space robotic systems. IEEE Transactions on Robotics and Automation, 9(5), October 1993.

[9] B. Eberman. Contact Sensing: A Sequential Decision Approach to Sensing Manipulation Contact Features. PhD thesis, MIT, May 1995. Dept. of Mech. Engineering.

[10] O. Egeland and J.R. Sagli. Coordination of motion in a spacecraft/manipulator system. Int. Journal of Robotics Research, 12(4):366-379, August 1993.

[11] P.M. Fitts. The information capacity of the human motor system in controlling the amplitude of movement. Journal of Experimental Psychology, 47:381-391, 1954.

[12] T.I. Fossen. Adaptive control of nonlinear systems: A case study of underwater robotic systems. Journal of Robotic Systems, 8(3):393-412, 1991.

[13] D.J. Hackman, N. Estabrook, H. Wheeler, and D. Uhler. Development of deepocean work system. In Proceedings of the IEEE 1975 Ocean Engineer Conf., 1975.

[14] N. Hogan. Impedance control: An approach to manipulation. Journal of Dynamic Systems, Measurement, and Control, 107, 1985.

[15] S. Jacobsen, D. Knutti, R. Johnson, and K. Biggers. Design of the utah/mit dextrous hand. In Proceedings of the IEEE International Conference on Robotics and Automation, 1986.

[16] D.M. Lane, J. Sneddon, D.J. O'Brien, J.B.C. Davies, and G.C. Robinson. Aspects of the design and development of a subsea dextrous grasping system. In Proceedings of OCEANS 94, volume 2, Brest, France, September 13-16 1994. 
[17] R.W. Longman, R.E. Lindberg, and M.F. Zedd. Satellite-mounted robot manipulators - new kinematics and reaction moment compensation. The International Journal of Robotics Research, 6(3):87-103, Fall 1987.

[18] M.T. Mason. Compliance and force control of computer controlled manipulators. IEEE Transactions on Systems, Man, and Cybernetics SMC-11, pages 418-432, June 61981.

[19] M.H. Raibert and J.J. Craig. Hybrid position/force control of manipulators. Journal of Dynamic Systems, Measurement, and Control, 102:126-133, June 1981.

[20] A. Rovetta. On some problems of design of multipurpose mechanical hands in industrial robots. In Proceedings of the 7th ISIR, Tokyo, Japan, 1977.

[21] S.I. Sagatun. Modeling and Control of Underwater Vehicles: a Lagrangian Approach. PhD thesis, Norwegian Institute of Technology, March 1992.

[22] J.K. Salisbury. Active stiffness control of a manipulator in cartesian coordinates. 19th IEEE Conference on Decision and Control, December 1980.

[23] J.K. Salisbury. Interpreting contact geometries from force measurements. International Symposium on Robotics Research, September 1984.

[24] C. Sayers. Operator Control of Telerobotic Systems for Real World Intervention. $\mathrm{PhD}$ thesis, University of Pennsylvania, 1995.

[25] J.M. Schimmels and M.A. Peshkin. Admittance matrix design for force guided assembly. IEEE Transactions on Robotics and Automation, 8(2):213-217, April 1992.

[26] F. Skinner. Designing a multiple prehension manipulator. Mechanical Engineering, September 1975. 
[27] E.R. Snow. The load/deflection behavior of pretensioned cable/pulley transmission mechanisms. Master's thesis, Massachussetts Institute of Technology, May 1994. Dept. of Mech. Engineering.

[28] N. Ulrich. Grasping with mechanical intelligence. Master's thesis, U. of Pennsylvania, December 1988. Dept. of Mech. Engineering.

[29] Z. Vafa and S. Dubowski. The kinematics and dynamics of space manipulators: The virtual manipulator approach. The International Journal of Robotics Research, 9(4):3-21, August 1990.

[30] G. Voellmer. Robotic gripper resists torsion and lateral forces. NASA Tech Briefs, 16(10):78, October 1992.

[31] G. Voellmer. A passive end effector change-out mechanism for on-orbit robotic servicing. Computers in Industry, 23:65-74, 1993.

[32] H. West. Kinematic Analysis for the Design and Control of Braced Manipulators. $\mathrm{PhD}$ thesis, MIT, 1986.

[33] D.R. Yoerger and J.J. Slotine. Robust trajectory control of underwater vehicles. IEEE Journal of Oceanic Engineering, OE-10(4), October 1985.

[34] D.R. Yoerger and J.J. Slotine. Adaptive sliding control of an experimental underwater vehicle. In Proceedings of the Int. Conf. on Robotics and Automation, Sacramento, CA, Apri 1991. 